Casale, Rita [Hrsg.]; Rendtorff, Barbara [Hrsg.]; Andresen, Sabine [Hrsg.]; Moser, Vera [Hrsg.]; Prengel, Annedore [Hrsg.]

\title{
Geschlechterforschung in der Kritik
}

Opladen ; Bloomfield Hills : Verlag Barbara Budrich 2005, 179 S. - (Jahrbuch Frauen- und Geschlechterforschung in der Erziehungswissenschaft; 1)

Quellenangabe/ Reference:

Casale, Rita [Hrsg.]; Rendtorff, Barbara [Hrsg.]; Andresen, Sabine [Hrsg.]; Moser, Vera [Hrsg.]; Prengel, Annedore [Hrsg.]: Geschlechterforschung in der Kritik. Opladen ; Bloomfield Hills : Verlag Barbara Budrich 2005, 179 S. - (Jahrbuch Frauen- und Geschlechterforschung in der Erziehungswissenschaft; 1) - URN: urn:nbn:de:0111-opus-53147 - DOI: 10.25656/01:5314

https://nbn-resolving.org/urn:nbn:de:0111-opus-53147

https://doi.org/10.25656/01:5314

in Kooperation mit / in cooperation with:

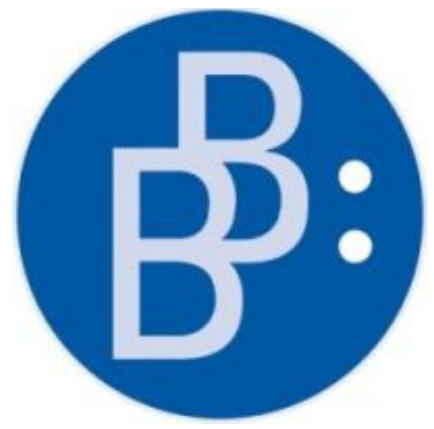

https://www.budrich.de

\section{Nutzungsbedingungen}

Gewährt wird ein nicht exklusives, nicht übertragbares, persönliches und beschränktes Recht auf Nutzung dieses Dokuments. Dieses Dokument is ausschließlich für den persönlichen, nicht-kommerziellen Gebrauch bestimmt. Die Nutzung stellt keine Übertragung des Eigentumsrechts an diesem Dokument dar und gilt vorbehaltlich der folgenden Einschränkungen Auf sämtlichen Kopien dieses Dokuments müssen alle Urheberrechtshinweise und sonstigen Hinweise auf gesetzlichen Schutz beibehalten werden. Sie dürfen dieses Dokument nicht in irgendeiner Weise abändern, noch dürfen Sie dieses Dokument für öffentliche oder kommerzielle Zwecke vervielfältigen, öffentlich ausstellen, aufführen, vertreiben oder anderweitig nutzen.

Mit der Verwendung dieses Dokuments erkennen Sie die Nutzungsbedingungen an.

\section{Kontakt / Contact:}

peDOCS

DIPF | Leibniz-Institut für Bildungsforschung und Bildungsinformation Informationszentrum (IZ) Bildung

E-Mail: pedocs@dipf.de

Internet: www.pedocs.de

\section{Terms of use}

We grant a non-exclusive, non-transferable, individual and limited right to using this document

This document is solely intended for your personal, non-commercial use. Use of this document does not include any transfer of property rights and it is conditional to the following limitations: All of the copies of this documents must retain all copyright information and other information regarding legal protection. You are not allowed to alter this document in any way, to copy it for public or commercial purposes, to exhibit the document in public, to perform, distribute or otherwise use the document in public.

By using this particular document, you accept the above-stated conditions of use. 
Geschlechterforschung in der Kritik 
Jahrbuch der

Frauen- und Geschlechterforschung in der Erziehungswissenschaft

herausgegeben von

Rita Casale

Barbara Rendtorff

Sabine Andresen

Vera Moser

Annedore Prengel

Folge 1/2005 
Rita Casale

Barbara Rendtorff

Sabine Andresen

Vera Moser

Annedore Prengel (Hrsg.)

\section{Geschlechterforschung in der Kritik}

Verlag Barbara Budrich

Opladen \& Bloomfield Hills 2005 
Gedruckt auf säurefreiem und alterungsbeständigem Papier.

Die Deutsche Bibliothek - CIP-Einheitsaufnahme

Ein Titeldatensatz für die Publikation ist bei Der Deutschen Bibliothek erhältlich.

Alle Rechte vorbehalten.

(C) 2005 Verlag Barbara Budrich, Opladen

www.budrich-verlag.de

\section{ISBN 3-938094-19-2}

Das Werk einschließlich aller seiner Teile ist urheberrechtlich geschützt. Jede Verwertung außerhalb der engen Grenzen des Urheberrechtsgesetzes ist ohne Zustimmung des Verlages unzulässig und strafbar. Das gilt insbesondere für Vervielfältigungen, Übersetzungen, Mikroverfilmungen und die Einspeicherung und Verarbeitung in elektronischen Systemen.

Umschlaggestaltung: disegno, Wuppertal - www.disenjo.de

Lektorat: Tina Jung

Satz: Verlag Barbara Budrich

Druck: DruckPartner Rübelmann, Hemsbach

Printed in Germany 


\section{Inhalt}

Grußwort der Vorsitzenden der Sektion Frauen- und

Geschlechterforschung in der DGfE

Editorial

\section{Beiträge}

Barbara Rendtorff

Strukturprobleme der Frauen- und Geschlechterforschung in der

Erziehungswissenschaft

Edgar Forster

Männerforschung, Gender Studies und Patriarchatskritik

Eva Breitenbach

Vom Subjekt zur Kategorie. Veränderte Denkfiguren

Fritjof Bönold

Zur Lage der pädagogischen Frauen- und Geschlechterforschung: bildungstheoretische Diskussionen

Susanne Maurer

Gedächtnisspeicher gesellschaftlicher Erfahrung? Zur politischen

Dimension von Frauen- und Geschlechterforschung

\section{Aus der Forschung}

Sabina Larcher

„Doing teacher" - professionelle Inszenierungen in Wechselwirkung von institutionellen Arrangements und sozialer Praxis

Marita Kampshoff

Geschlechterdifferenzen bei den Schulleistungen - empirische

Forschungen aus England und Deutschland im Vergleich 
Inga Pinhard

„Action is indeed the sole medium of expression for ethics “

Skizzen einer sozialen Ethik bei Jane Addams

\section{Rezensionen}

Christiane Micus-Loos

Rezension zu Eva Borst: Anerkennung der Anderen und das Problem des Unterschieds. Perspektiven einer kritischen Theorie der Bildung ...

Barbara Fontanellaz

Rezension zu Corinna Seith: Öffentliche Interventionen gegen

häusliche Gewalt. Zur Rolle von Polizei, Sozialdienst und

Frauenhäusern

Fritjof Bönold

Rezension zu Heide von Felden: Bildung und Geschlecht zwischen

Moderne und Postmoderne. Zur Verknüpfung von Bildungs-,

Biographie- und Genderforschung

\section{Ankündigung der nächsten Bände}

Band 2

Band 3

Über die AutorInnen 


\section{Grußwort}

Hiermit wird der erste Band des neuen „Jahrbuchs Frauen- und Geschlechterforschung" der Sektion Frauen- und Geschlechterforschung in der Deutschen Gesellschaft für Erziehungswissenschaft vorgelegt. Damit gibt sich die Sektion ein Periodikum, das die Forschungsthemen der Sektion der wissenschaftlichen Öffentlichkeit vorstellt. Die Reihe der Jahrbücher hat eine doppelte Aufgabe:

1. Eine produktive Auseinandersetzung unterschiedlicher Positionen der Frauen- und Geschlechterforschung im Diskurs;

2. Eine Dokumentation der Diskussion um das Thema Geschlecht in der Erziehungswissenschaft und ihren Teildisziplinen als Querschnittsthema.

In der Erziehungswissenschaft haben wir heute die Situation, dass viele AutorInnen die Gender-Perspektive aufgreifen und bei empirischen Studien den Faktor Geschlecht mit einbeziehen. Das ist insofern ein Erfolg, als die Relevanz der Kategorie Geschlecht als Strukturkategorie zur Analyse der Gesellschaft ins Bewusstsein der ForscherInnen gerückt ist. Dennoch ist es weiter sinnvoll, die feministische Perspektive der Frauenforschung (ebenso wie die Sicht der Männerforschung) und die kritische Analyse der weiblichen und männlichen Sozialisation in der theoretischen und empirischen Forschung in der Erziehungswissenschaft zu thematisieren. Die Problematisierung der gesellschaftlichen Bedingtheit konkreter Lebenslagen von Frauen und Männern in Geschichte und Gegenwart ebenso wie die empirische Erforschung aus einer Geschlechterperspektive heraus ist auch heute unabdingbar. Die bloße Diskussion der Daten zur Bildung und Sozialisation der Geschlechter in empirischen Studien ist nicht ausreichend.

Im Generationenverhältnis werden Themen der Forschung verändert, so auch die Geschlechterverhältnisse. Bildung und Sozialisation der Geschlechter wandeln sich an der Oberfläche der gesellschaftlichen Bedingungen. Eine Analyse der Geschlechterverhältnisse in ihrer Tiefenstruktur kann aber nur 
über die der feministische Erkenntniskritik und in historischer Perspektive geleistet werden.

Die erkenntniskritische und historische Position des Feminismus erlaubt die Einordnung einzelner Positionen im Zusammenhang. Ansonsten besteht die Gefahr, dass Wandlungen auf der Oberfläche der gesellschaftlichen Bedingungen in Bezug auf die Geschlechter zu falschen Schlüssen führen, etwa werden zum Beispiel die jüngst erstellten empirischen Ergebnisse zur beruflichen Sozialisation von Wissenschaftlerinnen so gedeutet, dass die Marginalisierung der Frauen an Hochschulen ihren Sozialisationsdefiziten geschuldet ist. Damit wird wie schon in den 60er Jahren zu Unrecht unterstellt, internale Faktoren der Frauen und nicht vor allem auch strukturelle Bedingungen seien die Ursache für Unterrepräsentation. Die gut verdeckten strukturellen Barrieren werden damit ausgeblendet.

Insofern können drei wissenschaftstheoretische Positionen aus der feministischen Wissenschaft heraus formuliert werden:

1. Feminismus als Erkenntnis leitendes Interesse, Wertbasis und Analyseinstrument für wissenschaftliche Forschung;

2. Wissenschaftliche Forschung zu den Geschlechterverhältnissen;

3. Politische Perspektive der Transformation der Gesellschaft in Richtung Geschlechtergleichheit, zum Beispiel durch Gender Mainstreaming.

Diese Positionen werden sich im vorliegenden und den kommenden Jahrbüchern nur Diskussion stellen.

Den Leserinnen und Lesern wünsche ich nun eine anregende Lektüre.

Hildegard Macha

Vorsitzende der Sektion Frauen- und Geschlechterforschung

im November 2004 


\section{Editorial \\ Geschlechterforschung in der Kritik}

Wir freuen uns sehr, unseren LeserInnen heute den ersten Band der neuen Jahrbuchreihe ,Frauen- und Geschlechterforschung in der Erziehungswissenschaft' vorstellen zu können.

Unser Anliegen mit dieser Reihe ist es, die aktuellen Erträge der erziehungswissenschaftlichen Frauen- und Geschlechterforschung vorzustellen, zu systematisieren und einem größeren Publikum bekannt zu machen.

In gewisser Weise hat die Frauen- und Geschlechterforschung inzwischen den Status einer ,Normalwissenschaft ‘ erreicht, was Publikationen, Lehrstühle, Forschungsmethoden und Diskussionszusammenhänge angeht. Die ,Normalisierung' der Geschlechterperspektive ist sozusagen zum Tagesgeschäft geworden und dokumentiert sich in der Publikation von Handbüchern, Monographien, Zeitschriften und Sammelbänden, sowie nun auch mit diesem Jahrbuch.

Wir betrachten dabei das Jahrbuch als ein geeignetes Medium, sowohl innovativ als auch aktuell die gegenwärtige Forschungslage zu begleiten und zu dokumentieren, als auch Impulse für Zukünftiges zu setzen. Insofern verstehen wir das Jahrbuch als einen strategischen Ort, Neues zusammenzuführen und zur Diskussion $\mathrm{zu}$ stellen sowie Themen $\mathrm{zu}$ initiieren und haben durch die redaktionelle Nähe zur Sektion Frauen- und Geschlechterforschung in der Deutschen Gesellschaft für Erziehungswissenschaft einen engen wissenschaftlichen Kontakt (hier möchten wir vorab auch auf das Grußwort von Hildegard Macha - Vorstand der Sektion Frauen- und Geschlechterforschung in der Erziehungswissenschaft - in diesem Band verweisen).

Auch will das Jahrbuch NachwuchswissenschaftlerInnen Raum geben, Forschungsarbeiten und Qualifizierungsprojekte vorzustellen, neue und möglicherweise etablierte Perspektiven zur Diskussion zu bringen und so dazu beitragen, den erreichten wissenschaftlichen Standard innerhalb der Frauenund Geschlechterforschung weiter zu entwickeln. Es geht uns also insgesamt um eine Theorie-geleitete Systematisierung der Geschichte der Geschlechter- 
differenz, ihrer Bedeutung für die heterogene Geschichte des Aufwachsens, für die Ausformulierung von Bildungs- und Erziehungstheorien, für Kindheits- und Jugenddiskurse sowie für die Etablierung der pädagogischen Professionen (siehe z.B. die aktuelle Diskussion der Feminisierung des Lehrerberufs).

Wenn wir Herausgeberinnen uns auch darin einig sind, dass die Hervorhebung des Geschlechts als strukturelle Kategorie das gemeinsame Programm dieses Jahrbuchs darstellen soll, so interpretiert doch jede von uns auf sehr unterschiedliche Art, wie die Strukturalität des Geschlechts zu fassen und zu entziffern sei. Uns erscheinen diese politischen und theoretischen Differenzen aber nicht als Hindernis, sondern als Herausforderung. Ohne die Differenzen zu leugnen, wollen wir sie programmatisch in der wissenschaftlichen Öffentlichkeit zur Diskussion stellen.

Dies sind Ziele, die mit einem Jahrbuch für Frauen- und Geschlechterforschung in der Erziehungswissenschaft verbunden sind und wofür ein diskursoffenes, disziplinär ausgerichtetes und transparentes Forum geschaffen werden soll. Die frühere Kommission und jetzige Sektion Frauen- und Geschlechterforschung hat sich gerade in den letzten Jahren bemüht, in ihren Jahrestagungen und Kongress-Symposien die theoretische Auseinandersetzung und Forschung stark zu machen, differenzierte Beiträge vorzulegen, um die Stimme der kritischen Geschlechterforschung in die erziehungswissenschaftliche Disziplin hinein hörbarer zu machen.

Diesen Impuls will und soll das Jahrbuch aufgreifen. Es soll die Kritiken, Einsprüche und Anregungen formulieren, die aus der Geschlechterforschung an die Disziplin adressiert werden, und es soll dazu beitragen, dass die Geschlechterperspektive weder als scheinbar privates Spezialinteresse einiger Wissenschaftlerinnen an den Rand geschoben wird, noch aber bei der Übernahme in die allgemeinen Diskurse der Erziehungswissenschaft allzu sehr domestiziert und neutralisiert wird.

Insgesamt hat die Frauen- und Geschlechterforschung der letzten zwanzig Jahre in erheblichem Maße dazu beigetragen, die strukturelle Bedeutung der Geschlechterordnung, die historische Gewordenheit geschlechtstypischer gesellschaftlicher Bedingungen und pädagogischer Maximen und die geschlechtstypisierenden Einflüsse in Erziehungs- und Bildungsprozessen zu analysieren und in die öffentliche Diskussion hinein zu tragen. Aufgrund ihrer politischen Geschichte hat sie aber in ihren ersten aktiven Forschungsjahren vorrangig an den Fokus der soziologischen Geschlechterforschung angeknüpft, bzw. an die Konzentration auf Benachteiligung und Unrechtsaspekte, auf Ausgrenzungs- und Abwertungserfahrungen von Frauen und Mädchen. 
Dieser dominierende soziologische Zugang hat für die Erziehungswissenschaft zur Folge gehabt, dass etwa die Koedukationsdebatte fast ausschließlich aus einer Gerechtigkeitsperspektive geführt worden ist, während z.B. Fragen nach möglichen geschlechtstypischen Lehr- und Lern-Strukturen, nach der Erklärung geschlechtstypischer Leistungsprofile usw. eher unterbelichtet geblieben sind, die Ansätze zu einer Geschlechterperspektive auf den Bildungsbegriff, die institutionelle Dimension von Erziehung o.ä. eher vereinzelt blieben und keine differenzierte Ausarbeitung erfuhren. Der disziplinbezogenen theoretischen Diskussion ist es selten gelungen, eine breite, gemeinsam geführte Auseinandersetzung mit den begrifflichen und theoretischen Grundlagen von Pädagogik und Erziehungswissenschaft zu initiieren - auch wenn diese Forschungsarbeiten und Debatten dazu geführt haben, dass die Disziplin insgesamt zumindest ansatzweise die Relevanz einer differenzierten theoretischen Reflexion und empirischen Forschung anerkannt und akzeptiert hat.

Im Gegensatz zu vielen interdisziplinären Ansätzen der Genderstudies ist uns hier daran gelegen, disziplinäre Forschungen vorzustellen und damit den notwendigen Diskurshorizont zu schaffen, um die Selbstverständlichkeiten der allgemeinen Erziehungswissenschaft zu befragen, zu irritieren und sie zu motivieren, ihre internen Selbstvergewissserungsdiskurse und laufenden Forschungsprojekte - seien es empirische Bildungsforschungen, Theorierekonstruktionen, Professionsforschungen oder auch methodisch-didaktische Konzeptionen - um eine Geschlechterperspektive zu erweitern.

Mit einer disziplinären Fokussierung der Diskussion sollen hier allerdings nicht die interessanten Ergebnisse einer langjährigen interdisziplinären Forschungspraxis der Frauen- und Geschlechterforschung ignoriert werden. Wäre das der Fall, würden sowohl bedeutende methodologische Erkenntnisgewinne als auch Erfahrungen aus akademisch relevanten institutionellen Etappen verloren gehen. Es ist in der Tat ein großer Verdienst der feministischen Forschung im internationalen Kontext gewesen, das erkenntnistheoretische Potenzial einer interdisziplinären Perspektive hervorzuheben und die interdisziplinären akademischen Strukturen u.a. mittels der Gründung von Graduiertenkollegs und der Herausgabe international angelegter Studien zu etablieren. Unsere disziplinäre Fokussierung möchte deshalb nicht als Rückfall hinter interdisziplinäre Forschungspraktiken verstanden werden, sondern will den erziehungswissenschaftlichen Gegenstands- und Problembezug in diesem Kontext stark machen. Denn nicht die akademischen Institutionalisierungen sollten als ,Kern' der Disziplin aufgefasst werden, sondern die Themen, Gegenstände, Probleme und Orte, die das pädagogische Wissen und die erziehungswissenschaftliche Praxis definieren. 
Erziehungswissenschaft hat immer mit dem Spannungsfeld von Autonomie und Abhängigkeit zu tun. Deshalb muss auch die erziehungswissenschaftliche Geschlechterforschung, unabhängig von der jeweiligen theoretischen Positionierung zur Geschlechterdifferenz, dieses Spannungsverhältnis kritisch reflektieren. Auch müssen beide, Erziehungswissenschaft und Geschlechterforschung, nach dem Subjekt in komplexen sozialen Bedingungen, seinen Erfahrungen und der Verarbeitung dieser Erfahrungen fragen, denn die Frage nach dem Verhältnis von Identität in kulturwissenschaftlicher Sicht und Ungleichheit in differenz- und sozialtheoretischer Sicht manifestiert sich in der Kategorie Geschlecht. Deshalb ist es unser Ziel, Frauen- und Geschlechterforschung nicht als ein Additiv zum bestehenden Forschungs- und Lehrkanon der Erziehungswissenschaft zu verstehen, sondern deutlich zu machen, dass jede erziehungswissenschaftliche Perspektive ein Geschlechterkonzept implizit schon enthält, welches wirksam wird, auch wenn es nicht offen zutage liegt: $\mathrm{Ob}$ nun von Bildung die Rede ist, von Kommunikationsstrategien, von Systembildungen, von habitualisierten Praxen, von Vermittlung oder Behinderung - immer ist auch Geschlecht als Distinktions-, Ordnungs- oder Strukturkategorie enthalten, und Frauen- und Geschlechterforschungen haben bereits ungezählte Beispiele angeführt, um dies deutlich zu machen. Doch seit etwa Mitte der neunziger Jahre lässt sich nach unserem Eindruck eine Veränderung in der öffentlichen Stimmung gegenüber Frauenforschung und Geschlechterthematik feststellen: Teilweise ist gereizte Zurückweisung zu vernehmen, teilweise offensives Desinteresse (,das Thema ist durch!'), teilweise wird die Geschlechterperspektive als ,Spiegelstrich', als mäßig wichtiger Zusatzaspekt eingeordnet. Damit wächst die Gefahr, dass die von der Frauenund Geschlechterforschung getragene Einsicht in die strukturelle Bedeutung von Geschlechterbeziehungen, die nachhaltige Wirkung von Geschlechterordnungen auf die Subjektentwicklung ebenso wie auf die institutionelle Dimension von Erziehung und Bildung wieder zurückgedrängt werden wird.

Neben der Analyse der strukturellen Aspekte der Geschlechterordnung macht Geschlechterforschung auch Aussagen über Individuen in Hinblick auf ihr Geschlecht. Dabei haben wir es, wie in allen anderen Aussagen, die über Menschen universell, gruppenbezogen oder individuell getroffen werden, mit Dilemma-Strukturen zu tun: Stets werden mit feststellenden Begriffen veränderliche und nie vollständig erfassbare Aspekte ihres Lebens benannt, seien es Kulturen und Subkulturen, Beeinträchtigungen, Generationen usw. Unsere Kategorien nähern sich in unvollendet bleibenden Bewegungen dem Leben der Individuen stets aufs Neue an. Dabei wechseln sich Hervorhebungen und Verflachungen von Differenz ab, und je nach politischen Situationen und Zie- 
len sozialer Bewegungen werden in einer Dynamik sich verändernder Denkfiguren Gleichheits- und Differenzvorstellungen betont oder kritisiert. Das macht für uns auch die Auseinandersetzung mit den internationalen Entwicklungen der erziehungswissenschaftlichen Geschlechterforschung interessant, sowie die Erforschung von Geschlechterverhältnissen und feministischen Theorien in einer international vergleichenden Perspektive.

Auch die historische Dimension der Genderfrage bezieht von hier aus ihre Wichtigkeit. In Bezug auf die Geschlechterforschung betrifft die Historizität nicht nur die historischen Formen der Geschlechterverhältnisse, sondern auch die Reflexion bzw. das Denken dieser Formen. Für die Bewegungen, die Verschiebungen des feministischen Denkens stellen - wie schon in Bezug auf die sozialpolitischen Voraussetzungen und Implikationen der Geschlechterforschung angedeutet worden ist - wissenschaftliche Diskurse und historische Kontexte ihre Entstehungsmöglichkeiten dar. Mit Blick auf die gegenwärtigen Bestrebungen einer Bildungsreform, so schleppend sie auch sein mag, ist das kritische Potenzial der Frauen- und Geschlechterforschung dringend notwendig. Derzeit geraten die traditionellen Verhältnisse von Bildung, Erziehung, Hilfe und Betreuung zunehmend in die Kritik, ohne dass sich bereits neue Perspektiven abzeichneten. Aufgrund ihrer Geschichte ist die Frauen- und Geschlechterforschung mit dem Verhältnis von Politik und Wissenschaft vertraut. Auch deshalb könnte aus einer systematischen geschlechtertheoretischen Position heraus die Spannung zwischen Bildungspolitik und Erziehungswissenschaft konstruktiv bearbeitet werden.

Die Redakteurinnen des Jahrbuchs wünschen sich, dass die Jahrbuchreihe dazu beiträgt, die theoretischen Debatten unter den Geschlechterforscherinnen selbst zu intensivieren - eine offensive kritische Auseinandersetzung unter den Geschlechterforscherinnen selbst kann u.E. nur positiv als Impuls für die Forschungsaktivitäten der Beteiligten und deren Wirksamkeit gesehen werden - wir werden uns deshalb auch bemühen, die Themen der Jahresbände an den wichtigen Bereichen von Geschlechterforschung, pädagogischer Praxis und pädagogischer Theoriebildung zu orientieren.

Die einzelnen Bände werden immer Beiträge zum jeweiligen Thema des Bandes, Berichte aus laufenden Forschungsprojekten und Rezensionen enthalten. In jedem Band soll es darüber hinaus die Möglichkeit geben, eine direkte Kritik oder Antwort auf einen Artikel des vorangegangenen Bandes zu veröffentlichen. $\mathrm{Zu}$ jeder dieser Rubriken können Beiträge bei den Herausgeberinnen des jeweils nächsten Bandes eingereicht werden. Um die Qualität der Beiträge zu sichern, haben wir dem Jahrbuch ein Review-Verfahren zugrunde gelegt und einen Beirat eingerichtet, dem WissenschaftlerInnen aus 
allen Teildisziplinen der Erziehungswissenschaft angehören - alle eingereichten Texte werden anonymisiert und in das Review-Verfahren gegeben.

Die in diesem ersten Band veröffentlichten Beiträge, in Form von Artikeln, Forschungsberichten und Rezensionen, stellen Ansätze dar, die existierende pädagogische Geschlechterforschung zu analysieren, zu kritisieren, weiter zu denken und zu entwickeln. Zur Diskussion in diesem ersten Band stehen Ergebnisse der erziehungswissenschaftlichen Geschlechterforschung, der Perspektivenwechsel von der Frauenforschung zur Geschlechterforschung und die politische Dimension dieses Übergangs. Außerdem ist eine erste Systematisierung der bildungstheoretischen Diskussionen zum Thema Geschlecht der letzten zwanzig Jahre zu lesen. Mit der Absicht, das Jahrbuch auch als Forum für die Auseinandersetzung mit der Männerforschung zu betrachten, haben wir für diese erste Nummer programmatisch entschieden, einen Text zu publizieren, der die Konfrontation zwischen Frauenforschung und Männerforschung historisch und analytisch ins Zentrum seiner Untersuchung stellt.

Das Thema dieses ersten Bandes Geschlechterforschung in der Kritik wird in dem Beitrag Strukturprobleme der Frauen- und Geschlechterforschung in der Erziehungswissenschaft von Barbara Rendtorff zum Gegenstand gemacht. Sie diskutiert die Strukturprobleme erziehungswissenschaftlicher Geschlechterforschung, die sich aus dem Verhältnis Erziehungswissenschaft und Geschlechterforschung als Wissenschafts- und Gesellschaftskritik ergeben. Einen spezifischeren Fokus auf die Geschlechterforschung entwirft Rendtorff mit der Analyse der vorfindlichen Genderkonzepte und ihrer impliziten Tendenz, ,Gender' lediglich als Beobachtungskategorie zu verwenden diese theoretische Schwäche zeige sich dann auch in den spezifisch erziehungswissenschaftlichen Geschlechterforschungen. Sie plädiert zuletzt für mehr „Rivalität“ zwischen Frauen, die sie als „Chance“ begreift, um der schwächenden Rezeptionssperre der Disziplin zu entkommen.

In Männerforschung, Gender Studies und Patriarchatskritik analysiert Edgar Forster den kritischen Zusammenhang zwischen Gender Studies Männerforschung und Patriarchat. Er thematisiert das Verhältnis zwischen feministischer Forschung und Männerforschung: diese habe von der Frauenforschung auf eine parasitäre Art wissenschaftlich und politisch profitiert. Deshalb habe sich eine kritische Selbstvergewisserung der Männerforschung sowohl mit den theoretischen Voraussetzungen ihrer Analysen als auch mit ihren politischen Ansprüchen bzw. Zielen auseinanderzusetzen. Diese Selbstvergewisserung wird von Forster in Bezug vor allem auf Donna Haraway und auf Jacques Derrida als „Positionierung“ gekennzeichnet. In Anlehnung an Althusser und Connell definiert er das Patriarchat als Ideologie, die von einer 
extremen Beweglichkeit charakterisiert sei, welche die akademische Männlichkeitskritik als eine Resouveränisierungsstrategie patriarchalischer Prägung erscheinen lasse.

In dem Artikel Vom Subjekt zur Kategorie. Veränderte Denkfiguren wird von Eva Breitenbach der Wechsel von der Frauenforschung zur Geschlechterforschung vor allem entlang der Konzeptualisierung von Macht und Identität unter einer konstruktivistischen Perspektive thematisiert. Sie unterscheidet Positionen und Problemlagen zunächst der Frauen- und dann der Geschlechterforschung archetypisch entlang des Problems der Gewalt, skizziert einen macht- und strukturtheoretischen Ansatz und anschließend das sozialkonstruktivistische Konzept des doing gender. Mit der Perspektive des doing gender, die hier vor allem am Beispiel der doing adolescence als erziehungswissenschaftlichem Fallbeispiel dargestellt wird, seien der Verfasserin zufolge die Fokussierung und Problematisierung der Geschlechterfrage von der Ebene des, warum' auf die des, wie' verschoben worden.

Fritjof Bönold bietet mit seinem Beitrag Zur Lage der pädagogischen Frauen- und Geschlechterforschung - bildungstheoretische Diskussionen einen Überblick über die bildungstheoretischen Diskussionen innerhalb der pädagogischen Geschlechterforschung der letzten 20 Jahre. Nach einer erfreulichen Feststellung einer ersten Traditionsbildung der Geschlechterforschung innerhalb der Disziplin, deren Wahrnehmung auf einer akademischen Ebene allerdings nur begrenzt sei, schlägt er eine Systematik vor, um die pädagogische Geschlechterforschung nach Phasen bzw. theoretischen Strömungen zu ordnen. Die Anfänge seien von den Debatten um die „Geschlechtsspezifische Sozialisation“, um die „weibliche Pädagogik“ und um die „Gleichheit und Differenz Problematik" charakterisiert. Dieser Phase folgte zu Beginn der 1990er Jahre eine selbstkritische Wendung, die von der Rezeption Butlers Analyse und von der Verbreitung ethnomethodologischer/interpretativer Forschungskonzepte verursacht worden sei. Nach der Verunsicherung der 1990er Jahre sei die bildungstheoretische Geschlechterforschung vor allem von einem dekonstruktivistischen bzw. poststrukturalistischen Ansatz geprägt worden.

In dem Artikel Gedächtnisspeicher gesellschaftlicher Erfahrung? Zur politischen Dimension von Frauen- und Geschlechterforschung von Susanne Maurer, der den Artikelteil des Jahrbuchs beschließt, wird vor allem die politische Dimension der Frauen- und Geschlechterforschung hervorgehoben. Es geht Maurer dabei um die Verortung dieser politischen Dimension im Dreieck Frauenbewegung, Feminismus und Frauenforschung. Die Verkopplung dieser drei Ebenen gelingt ihr dank einer doppelten Kennzeichnung des Feminismus als Kritik an „Entwertung, Enteignung, und Zerstörung weiblicher Existenz, 
Erfahrung und Arbeit“, und als „Denkbewegung“ bzw. als „grundlegende Erfahrung von Differenz". Als Gewinn des akademischen Feminismus seien vor allem eine Verflüssigung der Geschlechtergrenzen und der Aufbruch hierarchischer Strukturen zu verzeichnen; verloren gegangen seien dabei allerdings der nicht institutionell und disziplinär getrübte Blick sowie verschiedene kreative Wege der Erkenntnisgewinnung und des politischen Anliegens. Sie plädiert daher für die Kultivierung eines gesellschaftlichen Gedächtnisses auch in Hinblick auf die Frauen- und Geschlechterforschung.

Im zweiten Teil des Bandes berichten Marita Kampshoff, Sabina Larcher und Inga Pinhard aus ihren aktuellen Forschungen, die sich mit Lernprozessen, beruflichen und wissenschaftlichen Erfahrungen von Frauen innerhalb von pädagogischen Institutionen beschäftigen.

Marita Kampshoff vergleicht in ihrer Sekundäranalyse englische und deutsche empirische Schulstudien bezüglich des Geschlechterverhältnisses. Als Ergebnis hält sie fest, dass die deutschen Studien den Schulleistungsaspekt kaum untersuchen, die englischen Studien würden dagegen u.a. durch Methodendtriangulation zu differenzierteren Ergebnissen kommen, da sie auf diese Weise mehrere der o.g. Aspekte verknüpfen (wobei die PISA-Studie allerdings die Lernstile mit berücksichtigt hat).

Inga Pinhard arbeitet über das Konzept einer sozialen Ethik bei Jane Addams, der bekannten amerikanischen Sozialreformerin, Pädagogin, Pazifistin und Nobelpreisträgerin. Sie stellt ihre Überlegungen zum Verhältnis von individueller und sozialer Moral und zum Verhältnis von Moral in sozialer Praxis und in der Literatur vor und bezieht diese auf die aktuelle feministische Debatte um Ethik und Moralphilosophie.

Sabina Larcher untersucht BerufsanfängerInnen im Lehrerberuf hinsichtlich ihrer Auseinandersetzungen mit institutionellen Arrangements, den unterschiedlichen Erwartungen und ihrer professionellen Selbstinszenierung. Zwar arbeitet ihre Studie nicht ausdrücklich mit der Kategorie Geschlecht, bringt aber interessante Unterschiede in Bezug auf Sozialformen und dem Umgang mit Verantwortung hervor, so dass die Autorin das Konzept eines doing teacher als doing work while doing gender entwirft.

In der Rubrik, die den Rezensionen gewidmet ist, werden aktuelle Bücher von KollegInnen besprochen, die sich auf unterschiedliche Art in ihren Studien mit dem Thema des Bandes auseinandergesetzt haben. Für die nächsten Bände streben wir eine Erweiterung des Rezensionsteils an, auch um Besprechungen, die einen Blick auf internationale erziehungswissenschaftliche Diskussionen werfen. 
Und nun wünschen wir uns kritische und engagierte LeserInnen, und unseren LeserInnen eine interessante Lektüre!

Die Reihen-Herausgeberinnen

Sabine Andresen, Rita Casale, Vera Moser, Annedore Prengel, Barbara Rendtorff 



\section{Strukturprobleme der Frauen- und Geschlechterforschung in der Erziehungswissenschaft}

\section{Barbara Rendtorff}

Wenn der Ausdruck „Strukturen“ die Tatsache bezeichnet, dass es „,in der sozialen Welt selbst - und nicht bloß in den symbolischen Systemen, Sprache, Mythos usw. - objektive Strukturen gibt, die vom Bewusstsein und Willen der Handelnden unabhängig und in der Lage sind, deren Praktiken oder Vorstellungen zu leiten und zu begrenzen“" (Bourdieu 1992, S. 135), dann sind mit „Strukturproblemen“ solche Problemkonstellationen gemeint, die mit einer gewissen Notwendigkeit auftreten, sofern sie von jenen Anleitungen und Begrenzungen aufgeworfen werden.

Im Zusammenhang mit Erziehungswissenschaft und Geschlechterforschung von „Strukturproblemen“ zu sprechen, kann in zwei Richtungen verstanden werden: als Strukturproblem der Geschlechterforschung, die innerhalb der Disziplin Erziehungswissenschaft situiert ist und ihre Fragestellungen auf den erziehungswissenschaftlichen Themenkorpus richtet, und als Strukturprobleme des Verhältnisses zwischen der Disziplin und der Geschlechterthematik, der ,Tatsache des Geschlechts' und der aus einer Geschlechterperspektive vorgebrachten Theoriebeiträge und Kritiken. In beide Richtungen hat die Frauen- und Geschlechterforschung angesetzt, doch in beiden Richtungen besteht nach wie vor Diskussion- und Klärungsbedarf.

\section{Erziehung, Bildung und Geschlecht}

Wenn wir zunächst von der Frage ausgehen, was Erziehungswissenschaft überhaupt mit Geschlecht zu tun hat, so zeigen sich auch hier wieder zwei unterschiedliche Ebenen. Auf der Ebene erziehungswissenschaftlicher Begriffe und Konzepte lässt sich feststellen, dass Geschlechterbilder an zentraler Stelle eingelassen sind, auch wenn dies oft nicht auf den ersten Blick erkennbar ist. Die Kernbegriffe der Erziehungswissenschaft, Erziehung und Bildung, bilden als „unvereinbares“ Begriffspaar (Tenorth 1996, S. 43) ein für alle Pädagogik 
konstitutives Spannungsfeld zwischen der Anwaltschaft gegenüber dem Kind: den unvernünftigen, egoistischen, auf subjektive Freiheit und individuelle Entfaltung zielenden Wünschen des Individuums auf der einen, und dem Anpassungswunsch der Gesellschaft auf der anderen Seite. Der aber ist seinerseits auch in sich widersprüchlich, sofern er sowohl die begründete Forderung der Gemeinschaft nach Einfügung und Teilnahme des Einzelnen, nach Übernahme und Wahrung des Erreichten umfasst wie auch einen antizipatorischen Aspekt, den Wunsch der Gesellschaft nach Weiterentwicklung und die Erwartung, dass jede neue Generation aus dem Vorhandenen etwas Neues, ein ,Mehr' machen möge - durch seine Weiterentwicklung und folglich: seine Veränderung, Überwindung und Auflösung. Dieser Aspekt der Freiheit des Individuums, aus dem vorgefundenen gesellschaftlichen Wissen aktiv etwas unvorhersehbares Eigenes und etwas Neues zu generieren, das sich möglicherweise auch gegen intendierte Erziehungsziele wendet, wird im Deutschen im Bildungsbegriff aufbewahrt, der auch die Unplanbarkeit von pädagogischen Prozessen und eine konstitutive Spannung im Generationenverhältnis anzeigt.

Dieses Spannungsfeld sucht der gesellschaftliche Diskurs dadurch zu mindern, dass er die Komplexität nach zwei Seiten hin aufteilt und auflöst: der Komplex, der mit Einübung, Bezogenheit, Eingebundensein und Reifung zu tun hat, wird dem (privaten, familialen) Bereich der Erziehung zugeordnet, in dem die zielstrebige Einwirkung der Erwachsenen, der Gesellschaft auf das Kind grundsätzlich bejaht, ja sogar gefordert ist; Entwicklung, Herausforderung und Arbeit sind dem (öffentlichen) Bereich der Bildung zugeordnet, der entsprechend die Selbsttätigkeit und Eigenverantwortung des Heranwachsenden betont (vgl. z.B. Mollenhauer 1970, S. 65; Heydorn 1980, S. 8). Durch diese Teilung verschwindet die der Unplanbarkeit geschuldete, somit pädagogischem Handeln notwendig innewohnende Spannung gewissermaßen, zumindest verbirgt sie sich, die beiden Begriffsfelder werden vereindeutigt und der Natur des Gegenstandes geschuldet werden Mutter- und Vaterbilder unvermeidlich als typisierende Faktoren wirksam.

Die Erziehungstätigkeit scheint nun „,von der Natur diktiert“ zu sein, „da diese das Kind in einem Zustand der Schutzlosigkeit und der Ohnmacht auf die Welt bringt", und angesichts der Tatsache, „bei der Geburt ohne jedes Vermögen zu sein“, scheint die Freiheit für das Kind ohne Sinn zu sein (vgl. Merleau-Ponty 1994, S. 104). Erziehung hat somit einen starken Bezug zu einer kreatürlichen, und das heißt immer: einer mütterlichen Seite. Bei Heydorn hat sie ,archetypisch mütterlichen Charakter und weist auf die Einfügung in das Vorgegebene und Unabänderliche" hin (Heydorn, ebd.) und bei den meisten älteren Pädagogen finden sich ähnliche Formulierungen. Das zeigt sich 
dann etwa im Konzept der „Eignung“ für pädagogische Berufe (zentral für die Sozialpädagogik, die Vorschulerziehung oder das Grundschullehramt), wobei Empathie und Kindnähe als notwendig erachtet, aber überdeutlich geschlechtstypisch erwartet werden - die Systematik dieser Erwartungen und Zuschreibungen lässt sich sehr klar auch anhand von disziplinhistorischen Arbeiten nachvollziehen (vgl. z.B. Schmid 1989; Strotmann 1997; Rendtorff 2000), die auch offenlegen, wie sich fast unmerklich die Geringschätzung von Erziehung als einem privat-konservativen Bereich mit „bloßer Reproduktionsfunktion“ (Pongratz 1995, S. 20), einem „Domestikationszusammenhang“ (Mollenhauer 1976, S. 118) ihre Verortung im Privaten mit Frauen und mütterlichem Handeln verknüpft, während der väterlich-männliche Anteil eher an den Bildungsbegriff angebunden wird, sofern dieser mit Ermöglichung, Selbsttätigkeit, individueller Entwicklung und Freiheit konnotiert ist. Unnötig $\mathrm{zu}$ sagen, dass dadurch natürlich auch umgekehrt die Vorstellungen von männlichen und weiblichen „Charakteristika“, Fähigkeiten und Grenzen durch diese Konzepte gefärbt wurden. Die Wirkung von Geschlechterbildern im Bildungsbegriff zeigt sich deshalb weniger in Bezug auf seine instrumentelle, die Nützlichkeit und Verwertbarkeit betreffende, auf Optimierung von Kontexten und Transformation der Kontextur zielende Dimension (die „Aufgabenhaftigkeit" von Bildung), als darin, wie die Konstitution von Subjektivität und deren Bedingungen gedacht wird, die Verantwortlichkeit und Autonomie der Subjekte (vgl. Masschelein 1992, S. 92ff.), ihr Selbstverhältnis, zu sich und ihrem Handeln, und ihre Fähigkeit zu Modalisierung (Kokemohr 1985, S. 194f.), also die Fähigkeit, den Geltungsanspruch von Aussagen gegen die herrschende Konvention eigenständig zu variieren.

Gesellschaftliche Geschlechterbilder sind, wie wir wissen, als kulturelle Stereotype wesentlich binär organisiert um Kreatürlichkeit und deren Überwindung, um Bezogenheit und Getrenntsein, und als symbolisches System, als Geschlechterordnung bilden sie (wenn auch in je unterschiedlicher Form) ein zentrales Strukturmoment jeder menschlichen Gesellschaft. Deshalb müssen wir heute, nach aller historischen Erfahrung und den Erträgen aus dreißig Jahren Forschungen zu Geschlechterverhältnissen, davon ausgehen, dass die Wirkung der Geschlechterordnung zwar zunächst auf der Ebene der geschlechtstypischen Verteilung von Macht, Wertigkeit, Arbeit usw. sichtbar wird, dass aber diese nicht die zentrale Dimension ist, von der aus die Geschlechterordnung ihre persistente Kraft bezieht und von wo aus wir sie folglich analysieren müssen, um sie letztlich auch verändern zu können. Mag es auch unterschiedliche theoretische Modelle zu Struktur und Wirkungsweise der Geschlechterordnung geben - sie müssen in jedem Fall auf die Komplexität und Reichweite dieser Thematik antworten. 
Übertragen auf das Erziehungssystem als einer Partialgeschichte sozialen Wissens und sozialer Beziehungen (vgl. Hassauer 1994, S. 17) und die Akteure, die mit den Konzepten von Erziehung und Bildung hantieren bzw. innerhalb der von ihnen fundierten Institutionen arbeiten, müssen demnach Wirkungen auf die Struktur der familialen und außerfamilialen Erziehung, der Schule usw. erwartet werden. Dabei sind insbesondere die Wirkungen im Schulbereich von der Frauen- und Geschlechterforschung schon sehr früh in den Blick genommen worden, dennoch sind auch hier manche Zusammenhänge und Wirkungen nach wie vor weitgehend unbegriffen. Und das hat sicherlich mit der oben skizzierten Teilung zu tun. Die Beschäftigung mit der Geschlechterthematik stand (aufgrund ihrer Zuordnung zum weiblichmütterlichen Bereich) nie im Zentrum des Interesses der Bildungsforschung und ist im wesentlichen (bis heute) der Frauen- und Geschlechterforschung überlassen - wobei die älteren Forschungsarbeiten zudem durch den politischen Kontext des Ringens der Frauen um Gleichberechtigung gefärbt waren. Für die Pädagogik (als aufstrebende universitäre Disziplin) brachte diese Teilung den Vorteil, sich von einem schwierigen strukturellen Aspekt ihrer Materie zu entlasten. Denn die leise Herablassung, die Erziehungswissenschaft oftmals (von Seiten der Kopfdisziplinen) zu spüren bekommt, hat ja gerade damit zu tun, dass ihr der Stachel der Unplanbarkeit anhaftet, weil „das Geborenwerden in eine Welt der Grundbegriff ist, worauf die Erziehung beruht" (Masschelein 1996, S. 120), weil das Subjekt sich nicht fassen oder festlegen lässt, „weder von seinem Ursprung, noch von seinem Ziel her“ (Soëtard 1998, S. 280), was es der Erziehungswissenschaft nicht erlaubt, mit erwartbaren Ergebnissen pädagogischer Interventionen zu ,rechnen'. Es müsste von hier aus eigentlich nicht nur ein Bedürfnis der Frauen- und Geschlechterforschung sein, diese Zusammenhänge besser zu verstehen, sondern auch im Interesse der Disziplin insgesamt liegen, diese zu pointieren und zu theoretisieren, um offensiv mit der Problematik umzugehen. Die Spannung, die „Unvereinbarkeit“ zwischen Erziehungs- und Bildungsbegriff müsste dann weniger mit Blick auf die Unterscheidung zwischen einer ,gesellschaftlichen“ und einer „subjektiven“ Seite pädagogischen Wirkens (Tenorth, wie oben) diskutiert werden, sondern etwa als widersprüchliches Verhältnis zwischen Bezogenheit und Freiheit, zwischen Angewiesenheit und Autonomie im Menschenbild erziehungswissenschaftlicher Theorie, und dies eben auch sexuiert, d.h. bezogen auf Geschlecht, auf Mutter und Vater, auf die Konzepte von männlich und weiblich. Von hier aus stellt sich dann auch dringlich die Aufgabe, nicht nur die Wirkungsweise, sondern auch die Bedeutung der Geschlechterordnung für die gesellschaftliche Ordnung wie für die Subjektkonstitution besser zu verstehen. 
Es zeigt sich also, dass das Grundthema der Frauen- und Geschlechterforschung in der Erziehungswissenschaft keinesfalls den Charakter eines Gruppeninteresses oder einer Betroffenheitsliteratur hat, ,in der Mitglieder einer Bewegung die sie bewegenden Themen“" diskutieren (Drerup 1997, S. 855), und dass ihre Forschungsaufgabe sehr grundsätzlich angelegt ist und sich weder auf das Nachzeichnen der Einübung von Geschlechterpositionen im ,doing gender ${ }^{6}$ noch auf die Analyse von Ungleichheitsbeziehungen reduzieren lässt. Deshalb auch ist Forschung über Frauen oder Mädchen oder Praxen der geschlechtlichen Positionierung eigentlich erst dann ,Frauen- und Geschlechterforschung', wenn sie diesen Kontext auf eine grundsätzliche Weise mit thematisiert.

Wir können also als ein Strukturproblem der Frauen- und Geschlechterforschung festhalten, dass ihr Gegenstandsbereich wegen seiner, dem rationalen Wissen zumindest teilweise entzogenen, fundierenden Bedeutung für die symbolische Ordnung der Gesellschaft und der ihm innewohnenden Spannung zwischen historischer Veränderlichkeit und Persistenz der Grundkonstruktion nur schwer zu fassen ist, zumal ja die Instrumente seiner Analyse selbst der so gekennzeichneten Gesellschafts- und Denktradition entstammen.

\section{Erziehungswissenschaft und Geschlechterforschung}

Auch die Universität als Institution hat eine doppelte Aufgabenstellung: die der Reproduktion von Wissen, der Weitergabe anerkannter Übereinkünfte über Art und Ordnung dieses Wissens und die Formen seiner Bewahrung in akademischen Prozeduren (vgl. Nemeth 1996, S. 28), und zugleich die Aufgabe der Produktion neuen Wissens durch Infragestellen, Außerkraftsetzen und Überwindung des Übernommenen: ihr obliegt die „unbedingte“ und „voraussetzungslose Erörterung“" aktueller Probleme und die Erzeugung von neuem Wissen (Derrida 2001, S. 11ff.). Aber die Universität ist auch ein Ort gesellschaftlicher und Definitions-Macht, an dem es um die Vergabe von (hierarchisch gestufter) Anerkennung geht, von Positionen, die über das Operieren mit inneruniversitären Strukturen und Instrumenten hergestellt werden, und die neben dem eigenen Nutzen auch der Bestätigung dieses Rahmens dienen. Gestützt wird diese stratifizierende Positionierung über öffentliche Debatten oder wissenschaftskritische Einsprüche gegen die Formen des akademischen und universitären Betriebes selbst, sofern sie sich in öffentlich anerkanntem Terrain Gehör verschaffen können. Um im universitären Diskurs 
einflussreich zu sein, sind folglich Meriten in der wissenschaftlichen Fachwelt, in außerakademischen Medien und in den entsprechenden institutionellen Zusammenhängen gleichermaßen nötig.

In ihrer allgemeinsten Bestimmung verdankt sich die Frauenforschung einer Kritik, und zwar einer Kritik an gesellschaftlichen Strukturen und Machtverhältnissen als auch der daraus abgeleiteten Wissenschaftskritik. Diese Bestimmung als Kritik positioniert sie in der vorne skizzierten ambivalenten Aufgabe der Universität zwischen Reproduktion von Wissen und Produktion neuen Wissens sehr dominant auf der letzteren, lässt die Balance zu Einspruch und Veränderung hin sich neigen, wobei die Einsprüche nicht nur die Wissensbestände der Disziplin, sondern die Strukturen dieses Wissens selbst tangieren. Gerade deshalb waren Anregung und Unterstützung aus dem außeruniversitären bzw. außerakademischen Feld hier sehr wichtig, was der Frauenforschung den Ruch der Betroffenheitslyrik (Drerup) eingetragen hat. Dieser Herkunft wird im übrigen von der heutigen Sektion mit dem ersten Teil der Doppelbezeichnung „Frauen- und Geschlechterforschung“ Rechnung getragen.

Natürlich braucht jede Kritik, um sich wirksam entfalten zu können, den institutionellen Raum, an dem sich ihr Einspruch konkretisieren kann, und jedes Individuum braucht einen solchen Rahmen, um sich in Auseinandersetzung und Widerstand gegen dessen Zumutungen, dessen Homogenisierungsund Anpassungsdruck zu entwickeln, um individuelle Deutungen an der sozialen Realität zu messen, ,begründungsfähige Wirklichkeitsinterpretationen“ (Kokemohr 1985, S. 178) zu entwickeln und konfliktregelnde Verfahren einzuüben und zu erproben. Als akademisches Sprachrohr einer gesellschaftlichen Protestbewegung hatte die Frauen- und Geschlechterforschung deshalb zugleich immer auch das Ziel, ihre Vertreterinnen so zu positionieren, dass sie im Zentrum der Verwaltung und Tradierung des Wissens anerkannte Plätze einnehmen und damit ihrem kritischen Impuls (und auch ihren Forschungsergebnissen) Eingang in den „Reproduktions“-Bereich der Universität verschaffen konnten. Ob die Strategien der Frauenforscherinnen zur Unterstützung von Parteigängerinnen hier sinnvoll waren, oder ob tatsächlich die „,zu erfolgreiche“ Platzierung von „Berufsfeministinnen“ und ihr „Verstricktsein in die Techniken der Macht“ zu einer „Normalisierung“ im Foucaultschen Sinne (Holland-Cunz 2003, S. 169) geführt haben, also zu einer Einpassung in bestehende Machtverhältnisse und Konformisierung der Inhalte durch deren schleichende Einfügung in die bestehenden Strukturen, kann hier nicht weiter verfolgt werden. Festzuhalten bleibt aber, dass Frauen- und Geschlechterforschung dem Widerspruch nicht entgehen kann, einerseits als Einspruchsfigur in verändernder Absicht auf die Universität und ihre Wissensbestände zu zielen und zugleich in ihnen ,ankommen' zu wollen. 
Das eingangs angesprochene Strukturproblem der Geschlechterforschung innerhalb der Disziplin liegt also zu einem guten Teil darin, dass sie in den Kanon anerkannten Wissens aufgenommen werden will, um ihre Anerkennung zu dokumentieren und ihren Thesen Wirksamkeit $\mathrm{zu}$ verschaffen, zugleich aber doch außerhalb bleiben will, um ihr kritisches Potenzial zu bewahren und die Geste des ,Einspruchs“ beizubehalten. Und wenn sie versucht, dieses Dilemma nach einer Seite hin aufzulösen, so läuft sie in der einen Richtung Gefahr, sektiererisch und wirkungslos zu werden, und in der anderen Richtung würde die Abschwächung ihrer (wissenschaftskritischen und gesellschaftspolitischen) Einsprüche letztlich auch ihre Legitimität schwächen. Dieses Dilemma ist nicht zuletzt deshalb unlösbar, weil seine Ursache, das Geschlechterverhältnis selbst, in seiner komplexen Gestalt nach wie vor analytisch nicht wirklich aufgeklärt ist und als eine Frage den Themenstellungen der Geschlechterforschung unterliegt.

\section{„Gender" als Falle?}

Ein weiteres Strukturproblem der Frauen- und Geschlechterforschung liegt in den Begriffen, mit denen sie arbeitet. In ihren ersten Jahren war der Einfluss der Frauenbewegung auf die Forschungsfragen groß, war die Diagnose der rechtlichen und gesellschaftlichen Benachteiligung prominent und standen deshalb ,Frauen' (oder ,Mädchen') als Forschende und als Beforschte an erster Stelle. Nach und nach differenzierte und erweiterte sich dann die Perspektive und es wurde zu einer dringlichen Frage, wie die Kategorie „Geschlecht“ überhaupt aufzufassen und in ihrer Wirkung einzuschätzen sei: Was ist Geschlecht und was macht es mit den Menschen? Es entwickelten sich unterschiedliche theoretische Strömungen, z.T. mehr von der marxistischen Gesellschaftsauffassung geprägt, z.T. eher von psychoanalytischen, strukturalistischen oder anderen theoretischen Ansätzen geleitet, was zu großen theoretischen Unsicherheiten, einem ,erkenntnistheoretischen Durcheinander" (Scott 1994, S. 51) führte und zur Herausbildung von Meinungsführerschaften und konkurrierenden Lagern (vgl. z.B. die Kontroverse um „Kritik der Kategorie Geschlecht" in: Feministische Studien 2/1993). Ein zentraler Punkt war und ist in diesen Auseinandersetzungen die Frage, wie der Zusammenhang zwischen der unterschiedlichen physischen (morphologischen, genetischen) Ausstattung von Frauen und Männern und ihrer gesellschaftlichen Position aufzufassen ist. Solange dieser Zusammenhang als ganzer bei jeder einzelnen Forschungsfrage mit zur Debatte stand, war ,Geschlecht' als Kategorie oftmals 
als zu komplex und zu global, um sinnvoll damit zu arbeiten. So schien die systematische Unterscheidung zwischen Geschlechtskörper und Geschlechtsidentität, biologischem und sozialem Geschlecht, mithin zwischen sex und gender hier eine Erleichterung anzubieten.

Der Begriff ,gender“ hat, ähnlich wie „Differenz“ (vgl. Rendtorff 2004) eine Geschichte voller Missverständnisse und grundsätzlicher Widersprüche. Die amerikanische Sozialwissenschaftlerin Joan Scott definierte gender in den 1980er Jahren zunächst als ,das Wissen, das den körperlichen Unterschieden Bedeutung verleiht" (vgl. Nicholson 1994, S. 189). Damit war noch keine trennende Gegenüberstellung von gender und sex geboten - beide Begriffe grenzen ,eher einen problematischen Raum als eine saubere Unterscheidung“ ab, wobei der Begriff gender diesen Raum „,benennt“ (Eve Sedgwick, zit. bei Scott 2001, S. 56). Daneben gab es aber auch engere Definitionen, etwa als „Gruppe von Attributen“ für männlich und weiblich oder als „,sozial auferlegte Dichotomie von maskulinen und femininen Rollen und Charaktereigenschaften“ (vgl. Frey 2003, S. 26) und stärker kulturalistische Auslegungen, die gender als Ausdruck für kulturelle Regelsysteme und hierarchische Ordnungen (Stephan 2000, S. 68) verwenden und als Kultur-NaturOpposition der Kategorie sex gegenüberstellen. Eine dritte Variante gebraucht den Begriff gender gerade als Einspruch gegen diese Gegenüberstellung und betont, dass auch die physikalische Seite selbst ,,materiell und qualitativ durch die soziale Praxis verändert“ und „,zu etwas anderem“ werde (Lorber 1999, S. 60). Daraus wird (nicht nur bei dieser Autorin) umstandslos und m.E. vorschnell der Schluss gezogen, dass gender an kein biologisches Substrat gebunden (ebd., S. 66) bzw. sex eben auch gender sei (Butler 1991, S. 26 und passim; vgl. auch Müller 2001). Allen unterschiedlichen Autorinnen gemeinsam ist die Intention, nach der Bedeutung von Geschlecht für Gesellschaft, Kultur und Wissenschaft zu fragen und eine Begrifflichkeit zu finden, die es erlaubt, seine Funktionsweise im Prozess gesellschaftlicher Entwicklung zu erkennen, doch hat die Uneinigkeit zwischen diesen Positionen eine Weiterentwicklung der Gender-Theorie in den letzten zehn Jahren verunmöglicht (Waniek 2001, S. 147).

Letztlich hat sich heute wohl eine eher abgeschwächte und zugleich ausgeweitete Auslegung des Begriffs etabliert - zum Teil, weil dies eine Möglichkeit ist, Forschungen über Frauen ,ppolitisch akzeptabel zu präsentieren“ und „die akademische Seriosität einer Arbeit“" anzuzeigen (Scott 1994, S. 33), zum Teil, weil es die Forschungsaufgabe verkleinert und übersichtlicher macht, wenn es im wesentlichen um das „Wie“ einer Herstellung von Bedeutung geht. Das führt für die Praxis der Geschlechterforschung dazu, vorrangig „die Einprägung von ,Rollen“ (durch die männlich und weiblich endgültig in 
Mann und Frau überführt wurden) aufzuspüren, statt ein Projekt zu erfassen (nämlich die Schaffung einer festen und dauerhaften Opposition zwischen Mann und Frau), dessen Unmöglichkeit die Bedingungen seiner Umsetzung bestimmte" (Scott 2001, S. 41). So hat gerade die scheinbar so einleuchtende und hilfreiche Trennung und Gegenüberstellung von Geschlechtskörper und sozialer Geschlechtsidentität die Fragen nach dem ,Sinn', der konstitutiven gesellschaftlichen Bedeutung der Geschlechterordnung, ihrer Verankerung und Funktionsweise in Profession und Institutionen aus dem Blick genommen, sogar verdunkelt, weil das (irgendwie geartete) ,Interesse' der Gesellschaft am Funktionieren der Geschlechtsstereotype quasi implizit als gegeben vorausgesetzt wird.

Anfänglich hatten Frauenbewegung und Frauenforschung die Erklärung für die Ungleichgewichtigkeiten der Geschlechterordnung dem Zeitgeist folgend (ihrer Nähe zur Studentenbewegung und deren Orientierung an den politischen Strukturen der Arbeiterbewegung) in der Machtverteilung gesucht und sich, auch in ihren Forschungen, stark auf die Dimension „Benachteiligung“ konzentriert. Dabei gab es anfangs durchaus auch andere Ansätze, die der Vermutung folgten, die Geschlechterordnung sei Ausdruck einer grundsätzlicheren Problematik, die es zunächst zu erkennen gelte, um ihre performative und persistente Wirkmächtigkeit zu begreifen (vgl. z.B. die frühen Schriften von Irigaray oder Cixous; ein Sprachrohr dieser noch offenen Suche war die Zeitschrift Die Schwarze Botin). Doch diese Ansätze haben sich nicht zu einem starken Diskurs entwickeln können, sondern sind von der ,Sozialdemokratisierung ' der Frauenbewegung dominiert worden. Die pädagogische Frauenforschung suchte ihren Fokus in den Mechanismen von Erziehungs- und Bildungsprozessen, in Verteilungsaspekten, die Benachteiligungen erzeugen v.a. in Schule und Universität - und entwickelte logischerweise Konzepte „parteilicher“ Pädagogiken usw. Bis heute sind sozialisationstheoretische (und v.a. -praktische) Fragen nach der Erzeugung und Tradierung gesellschaftlicher Geschlechterbilder, gesellschaftliche bzw. pädagogische Vermittlungs- und kindliche Aneignungsprozesse der hauptsächliche Gegenstand erziehungswissenschaftlicher Frauen- und Geschlechterforschung. Die Konzentration auf gender-Aspekte hat die Fokussierung auf die gesellschaftliche Geschlechterposition und die mit ihr verbundenen Habitus- und Verhaltensaspekte verstärkt, sie hat eine scheinbare Klarheit geschaffen und „die Ambiguität völlig beseitigt, mit der die Phantasie die Subjektbezeichnungen ,Mann“ und ,Frau' sowie die Art und Weise, wie der Körper die Seele materialisiert, versieht" (Scott 2001, S. 40), wobei die strukturelle und die symbolische Seite der gesellschaftlichen Geschlechterordnung ebenso zu kurz kommen wie die Dimension der psychosexuellen Entwicklung des Kindes. 
Wie mir scheint, haben wir es nun mit einer Aufweichung der ursprünglichen Intention in zwei Richtungen zu tun: Zum einen wird der Begriff gender von einigen Autorinnen so weit gefasst, dass man seine analytische Kraft anzweifeln muss - doch die Rede vom doing und undoing gender oder genderbending (Braidotti 1994, S. 22) macht ja nur Sinn, wenn die begriffliche Grundlage geklärt ist. Die Konzentration auf das „,doing“ fungiert dann, wenn auch sicherlich ungewollt, als Verdeckungsgeste, wenn das zu findende Begründungsmuster als scheinbar schlüssige, einfache Prämisse schon vorher definiert und der empirischen Beobachtung des Gegenstands zugrunde gelegt wird, die damit letztlich zum Bestätigungsritual verkommt. Wenn die Gegenüberstellung von sex und gender ihr Verhältnis als beantwortet erscheinen lässt, muss es eben (scheinbar) nicht mehr thematisiert werden. So verzichten heute recht viele Arbeiten im Bereich der Frauen- und Geschlechterforschung auf eine Vergewisserung über diese Thematik - so z.B. auch in den neueren ethnomethodologischen Schulstudien (z.B. Faulstich-Wieland et al. 2004; Güting 2004) -, implizit ,,wird angenommen, es gäbe hier keinen Erklärungsbedarf bzw. der Begriff [gender] erkläre sich selbst“" (Frey 2003, S. 70). Die Frage nach der Bedeutung von Geschlecht wird auch dann weiter nivelliert, wenn das im weitesten (und manchmal vagen) Sinne konstruktivistisch verstandene doing gender in eine stetig wachsende Reihe anderer Zuschreibungen und Einübungsprozesse gestellt wird, wie doing ethnicity, doing student oder doing adult (vgl. Faulstich-Wieland et al. 2004, S. 24), auf gleicher Ebene nebeneinandergestellt und auch als einander ersetzend konzipiert, so dass etwa doing student zugleich auch als undoing gender verstanden werden kann.

Zum anderen liegt in der Verwendung von doing gender als ,Motiv“ empirischer Arbeiten das Risiko, dass Unterschiede zwischen Mädchen und Jungen so betont werden, dass man hier tatsächlich von einer Konstruktion sprechen muss - wenn etwa nur Lehrerin und Lehrer, Mädchen und Jungen kontrastierend verglichen werden, kann es im Grunde nicht verwundern, wenn die Autorinnen zuletzt nur alle bekannten geschlechtstypischen Verhaltensweisen bestätigt finden (vgl. z.B. Thies/Röhner 2000, S. 133). Die Problematik kontrastierender Gegenüberstellungen zeigt sich spätestens an manchen Handreichungen für den Unterrichtsalltag, wenn etwa die Aufforderung ergeht: „Nenne für jedes Fachgebiet herausragende Frauen und Männer. [...] Für jede gefundene Frau gibt es z.B. fünf Punkte, für jeden gefundenen Mann einen Punkt“" (Portmann 1999, S. 56).

Ich befürchte also, dass sich gerade für unser Themenfeld die breite Übernahme des Begriffs gender als eine Falle erwiesen hat, weil er zu Ver- 
kürzungen tendiert und es dem politischen und dem akademisch-universitären Diskurs gestattet, ihn seinerseits weiter zu verdünnen.

\section{Kritik und Selbstkritik}

Frauen- und Geschlechterforschung in der Erziehungswissenschaft hat ein sehr komplexes und nicht ganz einfaches Themenfeld zu bearbeiten. Weil das Geschlechterverhältnis ein komplexes gesellschaftliches Verhältnis ist, muss die Geschlechterforschung sich mit soziologischen Theorien befassen; weil es einen Identitätsaspekt hat, muss sie sich mit psychologischen Modellen auseinandersetzen; und die symbolische Dimension, die das Problem der Anerkennung oder der Andersheit berührt, macht die Beschäftigung mit philosophischen Ansätzen sinnvoll und notwendig. Wohl ist diese Angewiesenheit auf benachbarte Theoriefelder immer ein Problem der Erziehungswissenschaft gewesen - auch eine Theorie der Schule kann nicht ohne Soziologie der Institutionen auskommen, und muss doch über sie hinausreichen, eben in der $p \ddot{a}$ dagogischen Dimension des dort statthabenden Geschehens. Aber die Geschlechterforschung muss dieser voraussetzungsreichen Grundlagenarbeit eben noch einen ganzen Theoriezweig hinzufügen.

Mittlerweile blickt die Frauen- und Geschlechterforschung schon auf etliche fruchtbare Jahre zurück, auf theoretische und empirische Arbeiten, auf Anstöße zu Debatten: Die breit rezipierte Studie von Hagemann-White (1984) hat die Formel „Zweigeschlechtlichkeit als kulturelles System“ in den allgemeinen Diskurs eingebracht, die Studie von Prengel (1993) den Begriff „Pädagogik der Vielfalt“. Doch gibt es schon seit gut zehn Jahren auch deutliche Kritiken an der eigenen community, an Verkürzungen und falscher $\mathrm{Zu}$ friedenheit (vgl. z.B. Breitenbach/Hagemann-White 1994). Ein Blick über die Regalbretter mit erziehungswissenschaftlicher Geschlechterforschung lässt es durchaus wünschenswert erscheinen, dass sie ihren Blick und ihr Forschungsprogramm erweitern und ausdifferenzieren möge, und zwar nicht etwa nur, wie heute vielfach angemahnt, ausdehnen auf Jungen - das hat die feministische Literatur nämlich schon seit vielen Jahren als Forderung und Rat an die Disziplin herangetragen! - sondern vor allem, um mehr an den systematischen Grundlagen ihrer Thematik und ihrer Forschungsfragen zu arbeiten.

Wenn also heute in den Medien und der Disziplin mit dem Argument, die eigentlich vernachlässigte Gruppe seien doch die Jungen, der Geschlechterforschung im Nachhinein die Legitimität abgesprochen wird (vgl. stellvertre- 
tend den Spiegel-Titel „Schlaue Mädchen - Dumme Jungen. Sieger und Verlierer in der Schule“, Der Spiegel 21/2004 und die Themenbeilage Bildung und Erziehung der NZZ vom 20.1.2004), oder wenn Forschungsprojekte, Themenstellungen oder Lehrstühle für Geschlechterforschung abgewiesen werden mit dem Hinweis, das sei ja nun nicht mehr nötig, da Mädchen mittlerweile die besseren Schulkarrieren aufweisen, so müssen wir ehrlicherweise zugeben, dass die Frauen- und Geschlechterforschung zu diesem Dilemma selbst etwas mit beigetragen hat.

Einerseits hat sie zweifellos einen großen Wissenszuwachs über tatsächliche Verhaltensweisen im Kontext von Erziehung und Schule hervorgebracht: Wir wissen jetzt, wie viel mehr Mädchen als Jungen dieses oder jenes Spielverhalten bevorzugen, diese oder jene Fächer wählen, mathematische Lösungswege einschlagen oder Adjektive der Selbstzuschreibung auswählen. Aber andererseits wissen wir noch immer sehr wenig darüber, warum das so ist und was es bedeutet. Manche Themen wiederholen sich zudem oftmals, während andere Bereiche nach wie vor unbeforscht geblieben sind: Wir wissen fast nichts über geschlechtstypisierende familiale Erziehungsstile und deren Zusammenhang mit anderen Sozialisationseffekten; wir wissen wenig über die Logik pädagogischer Institutionen und deren systematische Geschlechterdimension; wir wissen wenig darüber, ob ethnie- und geschlechtsbezogene Stereotype zusammenwirken und wie sie das Lehrerhandeln oder die Leistungserwartung von SchülerInnen beeinflussen; wir wissen kaum etwas darüber, warum geschlechtstypische Leistungsprofile entstehen oder warum der Widerstand gegen die täglichen schulischen Anforderungen sich geschlechtstypisch unterschiedlich formt usw. Auch wenn es mittlerweile eine Menge (meist kleinerer) Detailstudien gibt - empirische Daten sind leider nicht selbsterklärend, und viele Studien lassen die Leserin letztlich ratlos zurück.

Natürlich kann man eine solche Kritik ohne weiteres auch gegen jedes andere Segment pädagogischer Literatur und Theoriebildung vorbringen. Aber da die Frauen- und Geschlechterforschung doch einer speziellen, durchaus missgünstigen Beobachtung unterliegt, hat diese Schwäche bedauerlicherweise zwei sehr abträgliche Effekte: Sie ist erstens ein immer wieder gerne genutztes Einfallstor für Kritiker, die das ganze Thema als unwissenschaftlich und altmodisch abtun möchten - die verdiente Kritik daran, dass und wie sie das tun, bleibt davon natürlich unberührt.

Der zweite Effekt ist eine wachsende Beliebigkeit. Alles, was irgendwie mit Frauen zu tun hat oder was Jungen oder Mädchen tun, kann heute, ohne sich seiner konzeptionellen Zusammenhänge zu vergewissern oder sich irgendwie auszuweisen, als „Gender-Forschung" deklariert werden. Das wie- 
derum trägt wieder zur Verundeutlichung des Gegenstands der Frauen- und Geschlechterforschung bei bzw. des Theoriesegments, das sie umfasst.

\section{Widerstände}

Das alles hat natürlich Gründe, und die liegen zu einem nicht unerheblichen Teil in dem beständigen Widerstand, den die akademischen Diskurse den Kritiken und Anregungen von Seiten der Geschlechterforschung ziemlich konsequent entgegensetzen: anfangs mit Spott und Abwehr, mittlerweile im Gewande kleinlicher sachlich-methodischer Kritik oder wohlwollend scheinender Subsumtion unter ihre eigenen Kategorien. Das gilt im übrigen nicht nur für die Erziehungswissenschaft - auch die Soziologinnen klagen z.B., dass Geschlechterfragen nicht zu den Hauptinteressen der Soziologie geworden, sondern „nachgeordnete Probleme, Gegenstand forscherischer Sonderinteressen, Spezialleidenschaft einer selbstreferentiell mäkelnden Minderheit" seien (Knapp/Wetterer 2003, S. 7). Auch das Bemühen der Geschlechterforschung, nach der ersten, wissenschaftskritischen Phase der Theoriebildung nun die ,Anschlussfähigkeit' zu betonen, hat die Rezeptionsbereitschaft kaum erhöht. Das Geschlechterthema ist nach wie vor weitgehend an die Person einzelner Frauen gebunden und sein Theoriebeitrag separiert - das führt dann nicht zuletzt dazu, dass „Sensibilität für Geschlechterverhältnisse“ als sechster Spiegelstrich in den Stellenausschreibungen auftaucht oder von einem Kandidaten ganz ernstgemeint gesagt wird, er habe „Geschlecht mit drin“. Auf diese Weise wird das Thema klein gemacht und bleibt, als immer ,zusätzliches', in seiner Wirkung begrenzt.

Eine Disziplin bzw. ein Diskurs hat grundsätzlich drei Möglichkeiten, auf die Zumutungen eines anderen Denkens zu reagieren (genauso wie ein Territorium auf die Zumutungen der Fremden): Entweder kannibalisch, indem er es sich durch Verschlingen, Einpassung, Vereinnahmung anverwandelt; zweitens, indem er es sich durch Immunisierung, Hegemonieansprüche und Ignoranz vom Leibe hält - beides sind gewissermaßen kontraphobische Selbstschutz-Strategien (vgl. Rendtorff 1998, S. 83ff.). Als drittes gäbe es die Möglichkeit, sich der Zumutung zu stellen und sich in der Konfrontation, in der Berührung mit dem Anderen selbst zu verändern, etwas Neues entstehen zu lassen. Das haben die akademischen Diskurse gegenüber dem Modernisierungsangebot aus der Geschlechterforschung in keiner Disziplin geschafft (am ehesten vielleicht noch in der Literaturwissenschaft). 
Es macht der Frauenforschung in der Erziehungswissenschaft immer noch zu schaffen, dass bei ihr eine Vermischung von wissenschaftlichen Fragestellungen mit Partialinteressen vermutet wird, und zweitens lassen sich nach wie vor mehr oder weniger deutlich geschlechtstypische Ressentiments blicken wenn etwa Dieter Lenzen den Grundschullehrerinnen attestiert, dass die Professionalitätserwartungen ihnen gegenüber kaum größer sein könnten als ,gegenüber Teilzeitkräften, die in Supermärkten als Lager- oder Kassierpersonal arbeiten“, da viele von ihnen die Funktion des Grundschullehramts als „Ergänzung zu ihren Verpflichtungen in der Familie“ übernehmen würden (Lenzen 2003, S. 484) - ,ihren“ Verpflichtungen! Weiter heißt es, dass das weibliche Selbstkonzept des Lehrers viel stärker auf Soziales und Pädagogisches ausgerichtet sei als auf professionelle Wissensvermittlung. Hier mutet die Entgegensetzung von Pädagogik und Wissensvermittlung doch sehr merkwürdig an - besonders wenn man bedenkt, dass Herr Lenzen dafür zum „Reformer des Jahres 2004" nominiert worden ist.

Wie weit übrigens die Widerstände im akademischen Betrieb auch Ausdruck einer Konkurrenzabwehr sind (denn der Einbruch der Frauen in die Domäne der Wissenschaft war ja durchaus eine Bedrohung gewohnter Strukturen), mag dahingestellt bleiben.

Forderungen nach Anerkennung oder Besserstellung von Gruppen lassen sich in einer Gesellschaft grundsätzlich dann legitimieren, wenn die jeweilige Gruppe sich als ein kollektives Subjekt konstituiert oder durch eine Besonderheit kennzeichnen lässt, die sie von der Masse der anderen Rechtssubjekte innerhalb der Gesellschaft unterscheidet: z.B. durch einen mangelnden $\mathrm{Zu}-$ gang zu materiellen Gütern (etwa Arme oder Arbeitslose) oder zu sozialem Schutz und Stütze (z.B. Waisenkinder oder Flüchtlinge) usw. Aber lassen sich „Frauen“ als eine Gruppe dieser Art auffassen? (vgl. Phillips 1991, S. 116ff.) Einerseits ja: sofern sie beispielsweise kulturelle, soziale, Status- oder Habitusformationen miteinander teilen und von der Gesellschaft zugeschrieben bekommen, die sie in gleicher oder ähnlicher Weise gegenüber männlichen Rechtssubjekten kennzeichnen und einschränken. Und zugleich nein: sofern sie in ihrer enormen Unterschiedlichkeit in Bezug auf Lebensumstände, Machtzugang, Bildungschancen und deren individuelle Verarbeitung eben nicht umstandslos unter dieser einen Kategorie zusammengefasst werden können. Das ist dann möglich und naheliegend, wenn die erfahrene Diskriminierung auf einer rechtlichen Ebene liegt und da auch verändert werden kann, aber es funktioniert nicht, wenn die Forderung darin besteht, die Besonderheit der jeweiligen Gruppe wertzuschätzen, denn das ist weder Gegenstand von Rechten noch ist es von Individualethik oder politischer Philosophie her be- 
gründbar. „Eine soziale Wertschätzung“, argumentiert z.B. Honneth, stellt nicht ein öffentliches Reaktionsmuster dar, das sich einklagen oder einfordern ließe“, bestenfalls könne man an die „Bereitschaft“ der hegemonialen Gruppe appellieren in der Hoffnung auf ihre „prozedurale Tugend“, die „Minderheiten als Anwärter einer sozialen Wertschätzung zu behandeln“ (Honneth 2003, S. 199f.).

Natürlich ist die Universität kein dem Staat oder der Gesellschaft vergleichbares Gebilde, und die Wissenschaft sollte anderen Regeln folgen als die Politik. Doch diese Überlegung auf unser Thema übertragend können wir festhalten, dass - soweit es eben aus rechtlichen und Grundsätzen der praktischen Ethik ableitbar ist - Frauen als Personen ihren Ort und ihre Anerkennung in der Universität (und der Disziplin) erwerben konnten, was sich aber nicht unbedingt auf die Wertschätzung der Ansinnen und Einwände der Frauen- und Geschlechterforschung erstreckt.

Vorher hatte ich argumentiert, dass die Geschlechterthematik an (i.d.R. weibliche) Personen gebunden bleibt - hier scheint es nun, dass im universitären Alltagsbetrieb die Personen (gewissermaßen auf einer rechtlichmoralischen Ebene) akzeptiert und in das ,Allgemeine' aufgenommen werden, nicht jedoch die von ihnen vertretene Thematik, die sozusagen verwaist zurückbleibt. Einer solchen Abtrennung leistet die Frauen- und Geschlechterforschung desto eher Vorschub, je enger sie ihre Thematik fasst und je mehr sie sie selbstreferenziell betreibt. Von hier aus würde sich die Notwendigkeit ergeben, die Geschlechterperspektive stärker auf alle, gerade auch auf die allgemeinen Fragen und Themen der Disziplin zu richten - weniger, um exklusiv geschlechtsbezogene Ausschnitte zu bearbeiten, als eher, um sie in die Reflexion jeder das Fach betreffenden Fragestellung einzuarbeiten.

Die Diskussion darüber, ob es politisch sinnvoller und wirkungsvoller sei, eigenständige Bereiche zu formen oder dezentrale Querstrukturen zu bilden, gibt es seit den 1980er Jahren, seit sich eigene Frauenplätze in Regierungen oder Kommunen etablieren konnten - und diese Frage stellt sich natürlich auch für den akademischen Bereich: Ist es die bessere Strategie, Frauenforschung, Gender-Studies usw. in eigenen Dependancen zu betreiben oder mit einer querliegenden, dezentralen Verortung? Die dezentrale Variante hat immer den Vorteil, dass die Thematik in alle Teilgebiete eingefädelt werden kann, aber auch den Nachteil, dass sie dort je einzeln auch schnell wieder zum Verschwinden gebracht werden kann. Insofern ist die dezentrale Lösung stärker von den Personen abhängig, die die Inhalte vertreten, während separate Gebilde immer Gefahr laufen, ihre Thematik exklusiv zu betreiben, was der Geschlechterthematik eigentlich langfristig nicht entspricht. 
Es bleibt also festzuhalten, dass Frauen- und Geschlechterforschung nach wie vor mit Widerständen von Seiten der Disziplin und der akademischen Diskurse rechnen muss, und zwar vielleicht eher in Form einer Rezeptionssperre als gegenüber ihren Vertreterinnen.

\section{Neue alte Fragen}

Ich denke nicht, bei aller Kritik und Selbstkritik, dass der Grund für diese Rezeptionsproblematik vorrangig in irgendwelchen Mängeln und Schwächen der Frauen- und Geschlechterforschung selbst zu suchen ist. Nicht, dass es die nicht gäbe! Aber mir scheint doch, dass die skizzierten Widerstände anderswo ihre Ursachen haben, und dass diese Ursachen etwas mit der Geschichte, der (symbolischen) Bedeutung und den Wirkungen der Geschlechterordnung zu tun haben.

Es erscheint heute schon fast unzeitgemäß, vielleicht ,uncool' und jedenfalls altmodisch, das Geschlechterverhältnis als eines gesellschaftlicher Ungleichheiten in den Blick zu nehmen, feministische Einwände gelten als überholt und unangemessen - aber mir scheint, wir müssten (bei allen in den letzten Jahren gewonnenen theoretischen und politischen Differenzierungen) ein wenig von jener, alten' Perspektive zurückerobern.

Ich befürchte, dass die Genderisierung der Debatte, bei der Auffassung von Gender als einem definierten Bündel von Zuschreibungen, die Perspektive zu stark verkürzt hat. Zudem hat sich wohl (auch unter Geschlechtertheoretikerinnen) die Auffassung durchgesetzt, dass gender etwas sei, das degendered werden kann - so dass die Frage, ob Geschlecht (als ,sex“, anders gesagt: als leibliche Existenz des Menschen) überhaupt eine Bedeutung habe (oder nur eine ,gendered category" sei), bereits beantwortet scheint und ein „transitives“ oder „multiples“ Gender-Konzept anvisiert wird (Frey 2003, S. 68), wobei ein wenig unklar bleibt, wie denn letztendlich eine gesellschaftliche Veränderung von hier aus zu bewirken sein kann (außer im Warten darauf, dass alle Individuen, mehr oder weniger einzeln, eine Wandlung ihrer Einstellungen vollziehen). Dabei ist die Frage m.E. tatsächlich erst nur im engen Sinne politisch beantwortet - dahingehend, dass ein (möglicherweise zu konstatierender) Einfluss der Leiblichkeit auf die individuelle Entwicklung keine Auswirkungen auf die rechtlichen und sozialen Lebensumstände, die Bildungs- und Berufsgeschichte haben darf. Damit ist aber erstens nicht gleichzeitig bewiesen, dass diese leibliche Existenz nicht auf irgendeiner Ebene Auswirkungen auf die psychische Existenzweise, das Körperbild 
oder die Identitätsentwicklung hat - auch wenn das in vielen Gender-Texten durch einfache Konjunktionen wie „also“ oder „folglich“ ausgeschlossen wird. Und zweitens ist damit nicht ausgeschlossen, dass die überwunden geglaubten Muster des Geschlechtsverhältnisses auf der politischen Ebene oder der symbolisch-politischen nicht weiterhin, dezent und unbemerkt, weiterwirken - vielleicht, weil die sie letztlich begründende Bedeutungsdimension von Geschlecht durch das de-gendering gar nicht berührt wird. Hier könnten auch mögliche Erklärungsansätze zu finden sein etwa für die beharrlich geschlechtsstereotypen Fächer- und Berufswahlen, die geschlechtstypischen Leistungsprofile oder die Persistenz der Muster familialer Arbeitsteilung. Mir scheint, dass in der Dethematisierung von Geschlecht sowohl ein wenig Resignation liegt als auch ein wenig Wunschdenken, dass die Problematik, über die nun so lange geredet und geschrieben worden ist, sich jetzt endlich fügen und von selbst verschwinden möge.

Da wir leider beherzt konstatieren müssen, dass das nicht der Fall ist, Beschreibungen keine Erklärungen sind und ohne Erklärungen auch zielstrebiges Handeln kaum möglich sein dürfte, bleibt es also nach wie vor die Aufgabe der Frauen- und Geschlechterforschung, nach Erklärungsansätzen zu forschen, die Unterschiedlichkeiten gelten lassen können, ohne sie deterministisch zu wenden oder in hierarchisierende Bewertungssysteme einzupassen, die aber die Kategorie, oder: die Fragestellung, die analysiert werden soll, nicht der Einfachheit halber von vorneherein verwerfen.

Wir haben in Erziehungswissenschaft und Pädagogik, ob in Praxis oder Theorie, immer mit Kindern, mit Menschen und ihren Identitätskonzepten zu tun, und wer einmal Kindern zugehört hat, weiß sehr gut, dass Papa, Mama, Pipi und Sexuelles die zentralen Themen kindlicher Phantasietätigkeit sind, dass Menschen die leibliche sexuelle Existenz, das rein und raus des Körpers, die Verbindung von männlich und weiblich in der Geburtigkeit des Menschen zum Ausgangspunkt ihres Selbstentwurfs nehmen und in der symbolischen Welt bzw. dem gesellschaftlichen Umfeld darauf die Antwort suchen. Sex und gender wären insofern nicht einander ausschließend gegenübergestellt und gender keine Definitions- oder Erklärungskategorie, sondern die je historische Auslegung dieses gesamten Komplexes. Deshalb würde ich die These sehr stark machen, dass eine Theorie der Geschlechterverhältnisse, oder der Bedeutung von Geschlecht, die diesen Umstand nicht reflektiert oder in ihr Modell integrieren kann, zumindest für unser Arbeitsfeld unbrauchbar ist. Ich bin aber auch sehr optimistisch, dass sich mit einem differenzierenden theoretischen Zugriff über die Geschlechterdimension von (pädagogischen) Institutionen, über Beziehungsstrukturen, Bildungsverläufe und Lebensentwürfe noch sehr viel mehr herausfinden lässt, als wir bisher wissen und meinen. 


\section{Rivalität als Chance}

Natürlich gibt es zu diesem Punkt auch ganz andere Auffassungen. Wie sich in den vorstehenden Überlegungen gezeigt hat, ist aber ,Inspiration durch Widerspruch` bzw. Respons und Widerpart, in Rivalität zu dem sich die Argumente schärfen und der Diskurs sich ausdifferenzieren kann, von außerhalb des Bereichs der Frauen- und Geschlechterforschung kaum zu erwarten. So müssen wir selbst füreinander Gegenüber und Gegnerinnen im Wettstreit der Theorien und Positionen sein, um eine reichhaltige, spannungsreiche Debatte führen zu können. Aber dem steht nicht nur der eigene, vorne beschriebene Wunsch nach Anerkennung innerhalb des Mainstreams entgegen, sondern auch ein wirksames Tabu über der ,Konkurrenz zwischen Frauenforscherinnen', das auch das produktive Rivalisieren um Gedanken, Theorien und Forschungsergebnisse affiziert. Diese Ambivalenz scheint mir mit verantwortlich zu sein für den Mangel an produktiver theoretischer Rivalität innerhalb der Frauen- und Geschlechterforschung. Denn Kritik als interessierte Auseinandersetzung hat zur Voraussetzung, dass man nicht gewärtig sein muss, abgestraft zu werden, und dass man sicher sein kann, dass sie die Diskussionspartnerin nicht beschädigt. Wenn aber aufgrund der akademischen Strukturen wissenschaftlicher Wettstreit mit Kolleginnen unfreiwillig als persönliche Rivalität erscheint, wenn man erwarten muss, der Anderen in ihrer ohnehin prekären inneruniversitären Position zu schaden, und zudem noch fürchten muss, dass die Kritik unter Geschlechterforscherinnen den schwachen Stand der gemeinsamen Thematik weiter schwächt, dann ist das wohl Grund genug, mit gegenseitiger Kritik zögerlich zu verfahren. Doch damit hätte die Geschlechterforschung sich dann letztlich doch der Rezeptionssperre der Disziplin und der akademischen Strukturen gebeugt. Die Zurückhaltung in Bezug auf gegenseitige Kritik ist also nicht unbedingt ein Zeichen von Einigkeit, aber bei aller Unterschiedlichkeit und allen wissenschaftlichen und theoretischen Differenzen haben wir doch eines gemeinsam, und das ist die politische Dimension des Gegenstandes, die entweder dem eigenen wissenschaftlichen Impuls entstammt oder der Thematik von der Rezeptionsseite hinzugefügt wird.

Ich würde mir deshalb wünschen, dass der Diskurs innerhalb der Frauenund Geschlechterforschung mutiger wird, genauer und offensiver, und dass wir gewissermaßen eine Selbstverpflichtung eingehen, theoriebildend $\mathrm{zu}$ arbeiten und nicht v.a. Beschreibungen zu liefern. Ich würde mir wünschen, dass wir händereibend zu einer Tagung anreisen, voller Vorfreude auf eine spannende Kontroverse mit dieser oder jener Gegenspielerin, auf Streit und Auseinandersetzung, aus der man voller neuer Ideen und Überlegungen an 
den Schreibtisch zurückkehrt - denn nur aus der Differenz, aus dem Zusammenprall mit dem Anderen entsteht ja etwas Neues. Wir haben es selbst in der Hand, und es gibt niemanden, der uns etwas abnimmt. Die selbstgestellte Aufgabe der Frauen- und Geschlechterforschung, zu einer veränderten zeitgemäßen erziehungswissenschaftlichen Debatte einen zentralen konzeptionellen Anstoß zu geben und so zu einer wissenschaftskritischen Erneuerung der akademischen Diskurse beizutragen, ist noch lange nicht abgeschlossen.

\section{Literatur}

Bauman, Zygmunt (1995): Postmoderne Ethik, Hamburg

Bourdieu, Pierre (1992): Rede und Antwort, Frankfurt a.M.

Braidotti, Rosi (1994): Gender und Post-Gender: Die Zukunft einer Illusion? in: Materialienband - Facetten feministischer Theoriebildung, Bd. 14

Breitenbach, Eva/Hagemann-White, Carol (1994): Von der Sozialisation zur Erziehung. Der Umgang mit geschlechtsdifferenter Subjektivität in der feministischen Forschung, in: Jahrbuch für Pädagogik, Frankfurt a.M.

Butler, Judith (1991): Das Unbehagen der Geschlechter, Frankfurt a.M.

Drerup, Heiner (1997): Die neuere Koedukationsdebatte zwischen Wissenschaftsanspruch und politisch-praktischem Orientierungsbedürfnis, in: Z.f.Päd. 43/1997

Faulstich-Wieland, Hannelore/Weber, Martina/Willems, Katharina (2004): Doing Gender im heutigen Schulalltag. Empirische Studien zur sozialen Konstruktion von Geschlecht in schulischen Interaktionen, Weinheim

Frey, Regina (2003): Gender im Mainstream. Geschlechtertheorie und -praxis im internationalen Diskurs, Königstein

Güting, Damaris (2004): Soziale Konstruktion von Geschlecht im Unterricht. Ethnographische Analysen alltäglicher Inszenierungspraktiken, Bad Heilbrunn

Hagemann-White, Carol (1984): Sozialisation: weiblich - männlich? Opladen

Hassauer, Friedreike (1994): Homo. Academica. Geschlechterkonstrakte, Institution und die Verteilung des Wissens, Wien

Heydorn, Heinz-Joachim (1980): Ungleichheit für alle. Zur Neufassung des Bildungsbegriffs. Bildungstheoretische Schriften, Band 3, Frankfurt a.M.

Holland-Cunz, Barbara (2003): Die alte neue Frauenfrage, Frankfurt a.M.

Honneth, Axel (2003): Umverteilung als Anerkennung. Eine Erwiderung auf Nancy Fraser. in: Fraser, Nancy/Honneth, Axel: Umverteilung oder Anerkennung? Eine politisch-philosophische Kontroverse, Frankfurt a.M.

Knapp, Gudrun-Axeli/Wetterer, Angelika (2003): Achsen der Differenz. Gesellschaftstheorie und feministische Kritik II, Münster 
Kokemohr, Rainer (1985): Modalisierung und Validierung in schulischen Lehr-LernProzessen, in: Ders./Marotzki, Winfried (Hg.): Interaktionsanalysen in pädagogischer Absicht, Weinheim

Lenzen, Dieter (2005): Diagnose Lehrer. Plädoyer für die Professionalisierung eines Berufsstands, in: universitas

Lorber, Judith (1999): Gender Paradoxien, Opladen

Masschelein, Jan (1992): Pädagogisches Handeln und Verantwortung. Erziehung als Antwort, in: Meyer-Drawe, Käte u.a. (Hg.): Pädagogik und Ethik, Weinheim

Masschelein, Jan (1996): Die Frage nach einem pädagogischen Grundgedankengang. Bemerkungen über Handeln und Pluralität, in: Ders./Wimmer, Michael: Alterität, Pluralität, Gerechtigkeit. Randgänge der Pädagogik, Sankt Augustin

Merleau-Ponty, Maurice (1994): Keime der Vernunft. Vorlesungen an der Sorbonne 1949-1952, München

Mollenhauer, Klaus (1970): Pädagogik und Rationalität, in: Ders.: Erziehung und Emanzipation, München

Müller, Cathren: Konstruktion und Rekonstruktion - Judith Butler revisited, in: Waniek, Eva/Stoller, Silvia (Hg.) (2001): Verhandlungen des Geschlechts. Zur Konstruktivismusdebatte in der Gender-Theorie, Wien

Nemeth, Elisabeth (1996): Institutionalisierte Illusionen: Forschung, Ausbildung und Bildung an der Universität, in: Universität, Bildung und Politik. Eine Bestandsaufnahme aus feministischer Sicht, Mitteilungen des Instituts für Wissenschaft und Kunst Wien, 4

Nicholson, Linda (1994): Was heißt ,gender"?, in: Institut für Sozialforschung (Hg.): Geschlechterverhältnisse und Politik, Frankfurt a.M.

Phillips, Anne (1995): Geschlecht und Demokratie, Hamburg

Pongratz, Ludwig (1995): Aufklärung und Widerstand. Kritische Bildungstheorie bei Heinz-Joachim Heydorn. In: Euler, Peter/Pongratz, Ludwig (Hg.): Kritische Bildungstheorie. Zur Aktualität Heinz-Joachim Heydorns, Weinheim

Portmann, Rosemarie (1999): Gleich verschieden. Beispiele für eine mädchen- und jungengerechte Koedukation, Wiesbaden

Prengel, Annedore (1993): Pädagogik der Vielfalt, Opladen

Rendtorff, Barbara (1998): Geschlecht und différance. Die Sexuierung des Wissens, Königstein

Rendtorff, Barbara (2000): Pädagogischer Bezug und Geschlechterverhältnis, in: Pädagogische Rundschau 6

Rendtorff, Barbara (2004): Theorien der Differenz - Anregungen aus Philosophie und Psychoanalyse, in: Glaser, Edith, Klika, Dorle, Prengel, Annedore (Hg.): Handbuch Gender und Erziehungswissenschaft, Bad Heilbrunn

Rössler, Beate (2001): Der Wert des Privaten, Frankfurt a.M.

Schmid, Pia (1989): Bürgerliche Theorien zur weiblichen Bildung, in: Hansmann, Otto/Marotzki, Winfried: Diskurs Bildungstheorie II, Weinheim

Scott, Joan (1994): Gender: Eine nützliche Kategorie der historischen Analyse, in: Kaiser, Nancy (Hg.): Selbst bewusst. Frauen in den USA, Leipzig 
Scott, Joan (2001): Überlegungen zu Geschlechtsidentität und Politik, in: Waniek, Eva/Stoller, Silvia (Hg.): Verhandlungen des Geschlechts. Zur Konstruktivismusdebatte in der Gender-Theorie, Wien

Soëtard, Michel (1998): Notwendige, gleichwohl unmögliche Gedanken zum Subjekt in den Erziehungswissenschaften, in: Winfried Böhm/Angelika Wenger-Hadwig (Hg.), Erziehungswissenschaft oder Pädagogik?, Würzburg

Stephan, Inge (2000): Gender, Geschlecht und Theorie, in: Braun, Christina v./Stephan, Inge: Gender-Studien: eine Einführung, Stuttgart

Strotmann, Rainer (1997): Zur Konzeption und Tradierung der männlichen Geschlechterrolle in der Erziehungswissenschaft, Berlin u.a.

Tenorth, Heinz Elmar (1996): Bildungsbegriff und Erziehungswissenschaft, in: Wilfried Böhm/Angelika Wenger-Hadwig (Hg.): Erziehungswissenschaft oder Pädagogik?, Würzburg

Thies, Wiltrud/Röhner, Charlotte (2000): Erziehungsziel Geschlechterdemokratie. Interaktionsstudie über Reformansätze im Unterricht, Weinheim

Waniek, Eva (2001): Bedeutung in der Gender-Theorie. Ein Beitrag zur Klärung eines Grundlagenproblems, in: Dies./Stoller, Silvia (Hg.) (2001): Verhandlungen des Geschlechts. Zur Konstruktivismusdebatte in der Gender-Theorie, Wien 



\title{
Männerforschung, Gender Studies und Patriarchatskritik
}

\section{Edgar Forster}

\begin{abstract}
„Was bleibt dem abstrakten Denker, wenn er klug und überlegt Ratschläge gibt? Also immer über die Wunde von Bousquet, über den Alkoholismus von Fitzgerald und von Lowry, über den Wahnsinn von Nietzsche und von Artaud sprechen und dabei am Ufer bleiben? Zum

Fachmann für solches Geplauder werden? Nur wünschen, daß jene, die geschlagen wurden, nicht allzu tief sinken? Nachforschungen anstellen und

Sondernummern herausgeben? Oder versuchen, sich darin ein wenig selbst zu sehen, ein bißchen Alkoholiker, ein bißchen verrückt, ein bißchen selbstmörderisch, ein bißchen Guerillero sein, gerade genug, um den Riß auszudehnen, aber nicht zuviel, um ihn nicht unwiderruflich zu vertiefen? Wohin man sich auch wendet, alles scheint trostlos. In Wirklichkeit aber:

Wie sich an der Oberfläche halten, ohne am Ufer zu bleiben? Wie sich und dabei die Oberfläche und die ganze Oberflächenorganisation einschließlich der Sprache und des Lebens retten? Wie zu dieser Politik, zu dieser vollkommenen Guerilla gelangen?“
\end{abstract}

(Deleuze 1993, 197)

Das Verhältnis von Männerforschung, Gender Studies und Patriarchatskritik wird im vorliegenden Beitrag einer kritischen Analyse unterzogen, denn ungeachtet ihrer fortschreitenden Institutionalisierung gibt Männerforschung wenig Auskunft über sich selbst: über ihr Selbstverständnis, über ihr Verhältnis zum Feminismus und zum Patriarchat, über ihr Verhältnis zu Politik und Praxis. ${ }^{1}$ Anders als die Men's Studies in den USA, Australien oder in den

$1 \mathrm{Zu}$ den wenigen kritischen Standortbestimmungen zählt der Text Offensichtlich männlich (Tillner/Kaltenecker 1995). 
skandinavischen Ländern verdankt sich die Ausbreitung der deutschsprachigen Männerforschung in den 1990er Jahren vor allem den Gender Studies, die an vielen Universitäten umstandslos feministische Theorien und Praxen abgelöst und damit Unterschiede zwischen feministischen Theorien, Männerforschungen, Queer Studies eher nivelliert oder zugedeckt statt zum Gegenstand kritischer Analysen gemacht haben. Männerforscher profitieren in akademischen Feldern von Kämpfen, die die zweite Generation feministischer Theoretikerinnen seit mehr als dreißig Jahren führen. Sie nützen ihre Bühnen und werden in einer historischen Phase ermutigt und unterstützt, ,die' Männerforschung als Forschungsfeld weiter zu entwickeln, in der Frauenforschung und Frauenpolitik auf vielen Ebenen wieder in Verteidigungspositionen gezwungen werden und unter Legitimationsdruck geraten.

Dieser Kontext ist für eine kritische Selbstvergewisserung unabdingbar, denn mein Interesse gilt der Frage, wie eine Männerforschung aussehen müsste, die gemeinsam mit feministischen Theorien und Praxen eine umfassende Geschlechterdemokratie vorantreibt - ein Ziel, das zwar allgemein akzeptiert, aber ausbuchstabiert und in der Praxis eingefordert auch innerhalb der heterogenen Forschungslandschaft von Männerforschern und in den verschiedenen Feldern von Jungen- und Männerarbeit Gegenstand heftiger Debatten ist.

Unter der vagen Bezeichnung ,Theorie und Praxis von Männlichkeitskritik" bündle ich das zunehmende Interesse auch von Männern, Männlichkeit zum kritischen Gegenstand humanwissenschaftlicher Forschung zu machen. Dabei handelt es sich bis heute nicht um eine dem Feminismus vergleichbare ,Bewegung', die nachhaltige Veränderungen der Wissenschaftslandschaft bewirkt hat. Was man absehen kann, ist eine allmählich einsetzende Rezeption internationaler Männerforschung, die Sensibilisierung für Männlichkeit in einer Reihe von praktischen pädagogischen Feldern mit einem starken Fokus auf Jungen- und Männerarbeit. Ich beschränke mich hier auf einige theoretische Aspekte derjenigen Männerforschung, die im deutschsprachigen Raum rezipiert wird ${ }^{2}$ und vernachlässige aufgrund der Ungleichzeitigkeit der Diskurse und der unterschiedlichen thematischen und methodischen Zugänge Strömungen in anderen europäischen und außereuropäischen Ländern. ${ }^{3}$

2 Vgl. einführend BauSteineMänner (2001), Brandes (2002), Connell (1999), Döge (2001), Döge/Meuser (2001), Walter (2000).

3 Vgl. einführend Haywood/Mac an Ghaill (2003), Whitehead (2002). 


\section{Männlichkeitskritik: Fünf Thesen}

Ich möchte fünf Thesen diskutieren und damit eine Positionierung der Männerforschung vornehmen.

○ These 1: Männlichkeitskritik ist eine theoretische Praxis des Eingriffs.

○ These 2: Männlichkeitskritik unterhält ein kritisches Verhältnis zum Begriff Identität.

- These 3: Für Männlichkeitskritik bleibt das ,Patriarchat' eine zentrale Analysekategorie.

○ These 4: Männlichkeitskritik ist weder Resouveränisierungs- noch Immunisierungsstrategie.

○ These 5: Männlichkeitskritik muss danach beurteilt werden, wie sie das Verhältnis zu feministischen Theorien und Praxen definiert.

\section{Positionierung}

Mein Nachdenken darüber, wie ich Männlichkeitskritik und damit meine eigene Forschungspraxis situieren möchte, hat eine ständige Referenz, ein wachsames und lustvolles Gegenüber: Donna Haraways Gedanken zum „situierten Wissen“. Aus diesem Text stammt auch der Satz: „Optik ist eine Politik der Positionierung“ (Haraway 1995, 86f.). Haraway argumentiert in ihrem Essay für die Verortung und Verkörperung von lokalisierbarem Wissen und verantwortbaren Erkenntnisansprüchen. Dieses Wissen ist utopisch und visionär. ${ }^{4}$ Aber Haraways Begriff der Vision bleibt noch mit der Körperlichkeit verschweißt und hat sich noch nicht in einen abstrakten, erobernden und unmarkierten Blick verwandelt, der Repräsentation erzeugt und ihr gleichzeitig entgeht. Da die Vision eine „Frage der Fähigkeit zu sehen“ und damit auch eine „Frage der unseren Visualisierungspraktiken impliziten Gewalt“ (ebd., 85 ) ist, bedeutet Vision immer eine bestimmte Art und Weise des Sehens. Die Vision hat ihren Wert in der partialen Perspektive, die sie ausdrücklich hervorhebt und anerkennt. Partialität heißt, man kann etwas auch anders sehen und muss sich für eine Sehweise entscheiden und für diese Entscheidung einstehen. So verbindet sich mit Haraways Begriff der Vision die Positionierung. 
Für Haraway ist Positionierung ${ }^{5}$ deswegen die entscheidende wissensbegründende Praktik, auf der Grundlage von Politik und Ethik. Eine visionäre Praktik entwickelt Sehpraktiken und Perspektiven, die nicht bereits im Voraus bekannt sind. Dabei bleibt sie aufmerksam für die Ränder des Sehens, für seine Ausschlussprozeduren, für das, was dem Sehen, das immer auch ein Fokussieren ist, entgeht.

Mit aller Vorsicht und das heißt gegen jede Absichtslosigkeit und Zufälligkeit führt Jacques Derrida das Wort position in seinen Text ein, das Dorothea Schmidt und Astrid Wintersberger mit „Setzung" übersetzen. Position hat bei Derrida eine präzise Funktion. Er benützt dieses Wort, um einen „Gestus aktiver Interpretation“ (Derrida 1986, 31) zu bezeichnen, der eine Reihe von Gesprächen über sein Werk charakterisiert. Die Positionierung gleicht einem ,Über-das-Ziel-Hinausschießen', denn eine Standortbestimmung des eigenen Werks kommt immer zu früh, sie ist immer unmöglich, da sie eine unabgeschlossene Arbeit rückblickend beenden und schließen würde. Deshalb hat es Derrida auch abgelehnt, in die deutsche Übersetzung der Gespräche, die vierzehn Jahre später publiziert werden, einzugreifen, denn jede nachträgliche Korrektur hätte eine absolute Autoritätsposition zum Text installiert: Das nachträgliche Zurechtrücken hebt den Autor in eine privilegierte Wahrheitsposition und suggeriert einen Ort der Wahrheit, von dem aus der Text ,richtig' gelesen werden kann. ${ }^{7}$

Bei Jacques Derrida (1986) kann man schließlich auch studieren, dass jeder Positionierung die Verausgabung eingeschrieben ist. Positionierung bedeutet, über das Ziel hinaus zu schießen; mehr zu sagen, als man weiß. Eine Positionierung öffnet das Feld, anstatt es abzuschließen, denn sie fordert heraus, eigene Positionen zu markieren, zu definieren - und damit sich auszusetzen und in Verhandlungen darüber einzutreten. Eine Position zu beziehen heißt nicht, der eigenen Forschung nachträglich Sinn einzuschreiben, sondern genau genommen: einen Akt zu setzen, einen Sprung zu wagen und einen Gedanken zu fixieren, der im Grunde durch seine Fixierung zu wirken beginnt, obgleich seine Fixierung ein Gewaltakt darstellt und zurückgenommen werden müsste, weil sie nicht haltbar ist. Aber sie löst nicht nur Kettenreaktionen aus, sondern sie zwingt sich zu verantworten und ist deshalb eine Praktik, die

Positionierung durchkreuzt das binäre Schema von Relativismus und Totalisierung, die sich spiegelbildlich zueinander verhalten (vgl. Haraway 1995, 84).

6 „Setzung“ - in der deutschen Ausgabe mit Anführungszeichen versehen, wohl um die Zurückhaltung und Schwierigkeit der Übersetzung angesichts des Bedeutungsüberschusses zu markieren.

7 „Dabei ist Derridas Lektüre Derridas in der gleichen Position wie unsere Lektüre Derridas.“ (Engelmann 1986, 17; Hervorhebung E.F.) 
der Ethik zuzurechnen ist. Sich zu positionieren ist eine Eingreifpraktik, eine theoretische Praktik, eine dekonstruktive Politik ${ }^{8}$

Düttmann (1992) weist auf die entgrenzende und verausgabende Kraft der Dekonstruktion hin. Notwendig sagt sie immer zu viel. Wenn es sich um Setzungen handelt, gibt es keine Begriffsapparate (vgl. Derrida 1986, 86). Sie funktionieren wie ein Supplement. Es (er-)öffnet das Feld, schießt über Grenzen hinaus, sprengt den Rahmen. Setzung ist „Setzung dieser Andersheit als solcher" (ebda., 177). In einem Brief an Houdebine (und dann im Gespräch mit Peter Engelmann) setzt sich Derrida (1986, 182f.) mit dem Wort position auseinander, und zwar mit „Setzung (der Andersheit)“: „1. Wenn die Andersheit des Anderen gesetzt (posée) ist, ja ausschließlich gesetzt ist, kommt sie dann nicht auf das Selbe zurück, zum Beispiel in der Form des ,konstituierten Objekts' oder des ,informierten Produkts', das mit einem Sinn versehen ist? Von diesem Gesichtspunkt aus würde ich sogar sagen, dass die Andersheit des Anderen in das Verhältnis das einschreibt, was in keinem Fall ,gesetzt ${ }^{\star}$ werden kann. Die Einschreibung, so wie ich sie in dieser Hinsicht definieren würde, ist nicht eine einfache Setzung: vielmehr das, wodurch jede Setzung durch sich selbst vereitelt wird (différance): Einschreibung, Markierung, Text und nicht nur These oder Themen-Einschreibung der These. [...] Und man kann den Begriff der Setzung (position) immer wieder umdefinieren, anhand desselben Wortes (Abzug, Verpflanzung, Ausweitung).“

Auf andere Weise zeigt Gilles Deleuze $(1997,101)$ in seiner Arbeit über David Hume die Bewegung einer verausgabenden Setzung im Verhältnis von Subjektivismus und Empirismus. „Es darf jedoch nicht übersehen werden, daß die Bewegung der Selbstentfaltung bzw. des Anders-Werdens eine doppelte ist: Das Subjekt geht über sich hinaus, das Subjekt reflektiert sich." Mit anderen Worten: „Glauben und Erfinden ist, was das Subjekt zum Subjekt macht" (ebda., 102). Schließlich besetzt die Einbildungskraft als Gegenstand und Methode der Kritik bei Dietmar Kamper $(1990,10)$ jenen Ort, von dem aus Antworten auf folgende Frage möglich werden: „Ist das, was einer mit seiner Erkenntnis anrichtet, ist überhaupt die Tatsache, daß einer so denkt, wie er denkt, mit Hilfe seiner eigenen Erkenntnis begreifbar?" Unübersehbar ist die Wiederaufnahme des Motivs der Nicht-Identität bei Adorno, die auf eine Theorie der Erfahrung zuläuft. Auch hier ergeben sich Parallelen zu Derrida $(1998,366,383)$ und zu Foucaults Der Mensch ist ein Erfahrungstier (1996). Erfahrung markiert in allen Fällen Kontingenz und Überschreitung.

8 „Warum soll man die Arbeit der Dekonstruktion aufnehmen, anstatt die Dinge so zu lassen, "wie sie sind? Usw. Nichts hier ohne ,Kraftakt', irgendwo. Die Dekonstruktion, darauf habe ich bestanden, ist nicht neutral. Sie interveniert" (Derrida 1986, 179f.). 
Dies ist die Bedingung für Veränderung, die noch nicht vollständig determiniert ist. Das aber bedeutet, dass wir in striktem Sinn genötigt sind, unmögliche Entscheidungen zu treffen.

\section{Eingriff}

Positionierungen und Setzungen anerkennen die partiale Perspektive und die damit verbundene Notwendigkeit, sich für eine Perspektive zu entscheiden und diese Entscheidung zu verhandeln. Positionierungen haben aber eine zweite Dimension: Jede Positionierung ist auch ein Eingriff in das Feld, das untersucht wird, denn die Vision, von der Haraway spricht, ist nicht nur die Fähigkeit zu sehen, sondern die Gewalt des Blicks zu sehen, indem der Blick auf sich zurückgeworfen wird, so dass er und seine Macht sichtbar werden. Weil der Blick und sein Feld nicht unabhängig voneinander existieren, weil also der Gegenstand einer Untersuchung mit seiner Repräsentation unauflösbar verknüpft ist, werden der Blick und das Subjekt des Blicks selbst zum Gegenstand der Untersuchung. Für Geschlechterforschung ist dies deswegen besonders bedeutsam, da der abstrakte, körperlose Blick ,die unmarkierte Position des Mannes und des Weißen" (Haraway 1995, 80) bezeichnet.

Deswegen lautet die erste These, dass Männlichkeitskritik eine theoretische Praxis des Eingriffs ist. Diese These enthält zum einen die Aussage, dass Theorie eine Aktivität darstellt, die die Beziehung zwischen Subjekt und Gegenstand der Untersuchung beschreibt, definiert und auch herstellt und damit das gesamte Feld produziert. Zum anderen bedeutet theoretische Praxis, ein Feld auf bestimmte Weise zu reartikulieren, also Verbindungen zwischen den Elementen eines Feldes herzustellen (und nicht nur abzubilden) (vgl. Hall 2000, 65). Würde sich Männlichkeitskritik als Männerforschung auf die ,blo-

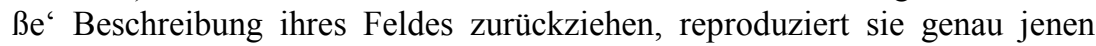
abstrakten phallozentrischen Blick, der selbst dem Gesehenwerden entgeht. In ein Feld einzugreifen bedeutet, die Macht des wissenschaftlichen Blicks sichtbar zu machen. In der Männerforschung paralysiert die Sprengkraft des Performativen, das die theoretische Seite des Eingriffs formuliert, das ,starke männliche Subjekt der Rede, das Selbstreflexion und Selbstkritik dazu nützt, Dominanz in verwandelter Form zu festigen. Männer erfahren dies für gewöhnlich so: Sie geben Positionen auf, sie stimmen der Kritik zu, dennoch bleiben ihre Positionen aus der Sicht feministischer Haltungen suspekt. Theoretisch geht es um die Frage, von welchem Ort aus männliche Subjekte spre- 
chen und - als kritische - sprechen können. Dabei geht es gerade nicht um eine politisch korrekte Position des Sprechens (wie manche Männer glauben machen wollen), sondern darum, die Position des Sprechens als eines männlich markierten Sprechens sichtbar zu machen - und damit die dem Eingriff eigene Form der Beschränkung und Verausgabung der theoretischen Aktivität.

\section{Who needs identity?}

„Wer braucht Identität?“ fragt Stuart Hall (1996) in einem Beitrag über das Verhältnis von Identität und Cultural Studies. Und wozu? Welche forschungsstrategische Bedeutung kommt der mit dem cultural turn verbundenen Wiederauferstehung des Begriffs Identität zu? Ähnlich äußert sich Lawrence Grossberg (1996a, 88): „Ich möchte zumindest die Frage stellen“, so Grossberg, „ob jeder Kampf auf dem Feld der Macht um Begriffe von Identitätsproblemen herum organisiert und verstanden werden kann und sollte und nahelegen, Identitätskonzepte neu zu formulieren." Grossberg interessiert sich dafür, wie Handlungsfähigkeit in spezifisch historisch-gesellschaftlichen Situationen hergestellt werden kann, wenn man nicht mehr davon ausgehen kann, dass es eine klare Trennlinie zwischen Mächtigen und Unterdrückten gibt und Widerstandspraktiken selbst in dem Machtfeld operieren, das sie bekämpfen. Dementsprechend stellt sich auch die Frage nach neuen Modellen politischer Gemeinschaften jenseits vorab festgelegter, stabiler Identitätskriterien.

Männlichkeitskritik unterhält, das ist meine zweite These, ein kritisches Verhältnis zum Begriff Identität. Das bedeutet, dass Männlichkeitskritik erstens danach fragt, was es bedeutet, Fragen von Männlichkeit und Männerpolitik als Identitätsfragen zu formulieren. Zweitens geht es um die Frage, wie Männerforschung das Verhältnis von Identität, Handlungsfähigkeit und Politik bestimmt. Ich werde die zweite These in drei Punkten artikulieren:

1. Zunächst werde ich die Funktion essentialistischer und nicht-essentialistischer Identitätskonzepte für die Männerforschung darstellen.

2. Dies mündet in den Vorschlag, die Begriffe „Interpellation“ (Althusser) und „Gabe“ (Derrida) für eine Geschlechtertheorie fruchtbar zu machen.

3. Im Anschluss an Grossberg werde ich schließlich diskutieren, wie eine Verschiebung der Grundlagen von Identität für Männlichkeitskritik genützt werden kann. 


\section{Essentialistische und nicht-essentialistische Identitätskonzeptionen}

Man kann, grob gesagt, zwei Paradigmen in der Identitätsdiskussion unterscheiden: ein essentialistisches und ein anti-essentialistisches Identitätsmodell. Zum ersten Modell zählen Formen personaler Identität, zum zweiten all jene Identitätsformen, die man auch als fragmentiert bezeichnen kann.

Personale Identität: Das erste Modell geht von einem unverbrüchlichen Identitätskern aus. Identität ist zeitüberdauernd und verbürgt Authentizität. Solche Identitätsmodelle haben in der deutschsprachigen Männerforschung und insbesondere in Theorien über Jungenarbeit ${ }^{9}$ eine ungeahnte Konjunktur. Unter dem Begriff personale Identität wird im Anschluss an die klassische Psychoanalyse und den Pragmatismus eine Tradition entwickelt, die (wie bei Erikson) Identität normativ entwirft und deswegen eng an Identitätskrise, Identitätsverlust und -diffusion anknüpft. Daraus speist ein Zweig in der Männerforschung und Männerarbeit immer wieder ihre Energie: Männliche Identität ist ständig bedroht, sie muss immer wieder neu erarbeitet und aufrechterhalten werden. Sie ist - per definitionem - krisenanfällig. Straub $(1998,95)$ nennt moderne Lebensbedingungen als unausweichliche Gefahr für Identität. „Als ein solches Konstrukt ist es etwas permanent Gefährdetes.“ Als Ursachen für männliche Identitätskrisen entdecken Männer regelmäßig den Feminismus, Frauen, die sich von ihren traditionellen Geschlechterpositionen emanzipieren, die Allmacht der Mütter, aus deren Abhängigkeit sich Jungen nicht lösen können, schließlich die angebliche Benachteiligung von Vätern in Scheidungsverfahren. Wenn sich Männlichkeit als relationaler Begriff über Weiblichkeit definiert, dann wird jede reale Veränderung von Geschlechterverhältnissen zu einer Gewinn-Verlust-Rechnung. Was Frauen gewinnen, verlieren Männer und die Emanzipation von Frauen ist dann unauflösbar mit männlichen Identitätskrisen verknüpft und nur noch als Bedrohungsszenario wahrnehmbar. Männliche Initiationsrituale sollen als besondere Facette männlicher Identitätsdebatten eine starke Männlichkeit unabhängig von unkalkulierbaren Wechselfällen der Geschlechterpolitik sichern. Bekannt geworden ist die mythopoetische Männerbewegung à la Robert Bly (1991). Jungen- und Männerarbeit sowie Männerforschungen, die daran anschließen, diagnostizieren Männer in der Krise und setzen auf starke Männlichkeit.

9 Eine Alternative dazu bietet Olav Stuve (2001), der Jungenarbeit mit Queer Theory verbindet (vgl. auch Bieringer/Buchacher/Forster 2000). 
Fragmentierte Identität: Das zweite Modell formuliert Identität nicht-essentialistisch. Es behauptet im Wesentlichen die Unmöglichkeit einer völlig konstituierten, von anderen klar unterschiedenen Identität. In den Cultural Studies sind diese Identitätsformen eng mit den Begriffen Fragmentierung, Hybridität und Diaspora verknüpft. Theorien über nicht-essentialistische Identitäten sind für die Diskussion des feministischen Subjekts in den 1980er Jahren, die mit Judith Butlers Gender Trouble ihren Höhepunkt und - vorläufigen - Abschluss erleben, zentral.

Für Männlichkeitskritik lassen sich im Anschluss an Butlers Ausarbeitung einer performativen Subjektkonzeption eine Reihe von Fragen stellen. Meine Bemerkungen und Fragen zu Butler sind im Gegensatz zu vielen Positionen in der Männerforschung ${ }^{10}$ nicht vom Wunsch getragen, poststrukturalistischen Positionen die Spitze zu brechen, sondern Männlichkeitskritik auf der Grundlage poststrukturalistischer und dekonstruktiver Theorieansätze weiterzuentwickeln.

○ Die deutschsprachige Männerforschung und Männerarbeit fällt zusammen mit der Transformation feministischer Theorien und der Durchsetzung von Gender Studies. Ich denke, dass Männlichkeitskritik diesen Umstand nicht außer Acht lassen darf und das Verhältnis von Männerforschung und Feminismus artikulieren müsste (vgl. unten).

- Butler hat zugespitzt formuliert, dass man nicht von vornherein eine Kategorie „Frau(en)“ annehmen kann und dass auch eine universale Struktur des Patriarchats problematisch sei. Wenn es die Kategorie „Frau(en)“" nicht gibt, so könne man auch nicht von den Männern (als Kategorie) sprechen, behaupten Georg Tillner und Siegfried Kaltenecker (1995). Sie halten die repräsentative Kategorie Männlichkeit für eine patriarchale Fiktion, die eine bindende (hegemoniale) Kraft für Männer entfaltet, die dem niemals erreichbaren Ideal von Männlichkeit nacheifern. Der Widerspruch zwischen dem Fan-

10 Robert W. Connell (1999) erwähnt sie im deutschen Standardwerk Der gemachte Mann. Konstruktion und Krise von Männlichkeiten überhaupt nicht und im neueren Buch The Men and the Boys nur am Rande, um ihr Performativitätskonzept zurückzuweisen (vgl. Connell 2000, 20). Holger Brandes (2002, 57ff.) diskutiert Butler kritisch. Problematisch sei, dass Butler ,den Ort der Praxis falsch lokalisiert, indem sie soziale Praxis auf Diskurs und Text reduziert“”, „Soziale Praxis schließt“, so Brandes (ebd., 60f.), ,nämlich durch den Blick auf die Ebenen des Handelns in sozialen Kontexten den Körper und alle seine sinnlichen Qualitäten unmittelbar mit ein." Diese ,verkörperte“ soziale Praxis findet Brandes bei Connell und bei der Bourdieuschen Habitus-Theorie, die Brandes - der Titel seiner umfangreichen Arbeit, nämlich Der männliche Habitus, kündigt dies bereits an - die theoretische Grundlage seiner Männerforschung bietet. 
tasma einer vollen Männlichkeit und dem gelebten Mann-Sein erklärt sowohl männliche Gewalt, eröffnet aber auch ein Feld für andere Männlichkeitsstrategien. Die zweite Schlussfolgerung, die man aus der Transformation der Butlerschen These ziehen kann, ist die Zerstörung der Kategorie Mann selbst. Es gibt viele unterschiedliche männliche Lebensentwürfe, die nicht mehr durch eine gemeinsame Struktur verbunden sind. Damit verwandeln sich männliche Lebensentwürfe in bloße' Lebensentwürfe. Man mag zwar noch von hegemonialer Männlichkeit sprechen, aber sie ist allenfalls ein Bild, dem Männer nicht mehr notwendig unterworfen sind. Sie treten aus dem Männerbund aus und legen ihre Verantwortung für das Patriarchat ab. Eine latente Struktur, die die Gemeinsamkeit zwischen Männern als Männer herstellt, wird unterschlagen und damit geht der Blick für die fundamentale Ungleichheit zwischen den Geschlechtern zugunsten individueller Subjektpositionen verloren (vgl. Meuser/Behnke 1998, 12f.). Wenn man nun auch noch Butlers (vgl. 1991, 18) These, dass eine Frau zu ,sein', nicht alles ist, was man ist, in die Männerforschung übersetzt, wird Geschlecht als Strukturkategorie endgültig marginal. Ich werde diesen Faden mit der dritten These über das Patriarchat als Analysekategorie wieder aufnehmen. Hier ist zunächst zu beachten, dass Butler darauf insistiert, dass die Handlungsfähigkeit gerade nicht an ein herkömmliches, starkes feministisches Subjekt gebunden ist. Bedeutet nun die Dekonstruktion der Kategorie Mann den Abschied aus feministischer Politik? Folgt daraus schlicht die Negierung des Patriarchats? Und welche Bedeutung und Funktion hat dieses Patriarchat? Lässt es sich auch unter der Bedingung formulieren, dass es den Mann nicht gibt? Ist es möglich aus der Sicht von Männlichkeitskritik die Begriffe von Identität und Patriarchat zu reformulieren, die nicht hinter die Einsichten von Subjektkritik und Patriarchatskritik zurückfallen, ohne damit zugleich die Basis für Männlichkeitskritik aufzugeben? Was also bedeutet Butlers (1991, 35) Kritik, dass es falsch sei, ,von vornherein anzunehmen, dass es eine Kategorie ,Frau(en)“ gibt“, für männlichkeitskritische Forschungen? Sind auch die Kategorien „Mann“ und „Männer“ unvollständige Kategorien, die ,als stets offener Schauplatz umkämpfter Bedeutungen“ (ebda.) dienen?

- Ein zentraler Aspekt der Butlerschen Dekonstruktion der sexgender-Dichotomie führt zu folgender Schlussfolgerung: „Die Begriffe Mann und männlich können dann ebenso einfach einen männlichen und einen weiblichen Körper bezeichnen wie umgekehrt die 
Kategorien Frau und weiblich" (Butler 1991, 23). Muss man sich demzufolge von der Differenz Mann/Frau verabschieden und stattdessen von weiblich und männlich markierten Subjektpositionen ausgehen? Donna Haraway $(1995,76)$ formuliert ihr Unbehagen unmissverständlich: „Diese Welt-als-Kode [...] ist ein hochtechnisiertes militärisches Feld, eine Art automatisiertes akademisches Schlachtfeld, in dem Leutpunkte, sogenannte Spieler, sich gegenseitig auflösen (was für eine Metapher!), um im Spiel um Wissen und Macht zu bleiben." Ist die Konstruktion von Subjektpositionen die einzige Möglichkeit, essentialistischen Fallen zu entgehen? Und was ist am Essentialismus die Falle? Um mit Haraway zu sprechen: Genügt es zu sagen, dass es theoretisch nicht ausgeschlossen werden kann, dass wir einen männlichen Körper mit dem verbinden, was wir Weiblichkeit nennen und umgekehrt, weil Weiblichkeit und Männlichkeit Konstruktionen sind? Würde das nicht dazu führen, dass kulturellen Konstruktionen letztlich eine Kraft zukommt, die einst die Biologie hatte? Als Naturalisierung wird die kulturelle Konstruktion als Schicksal erlebt, während es doch darum ginge, das Gesichtsfeld zu weiten, um anders zu sehen.

\section{,Gegebenes` Geschlecht}

Poststrukturalistische Identitätskonzeptionen wie diejenige von Judith Butler rufen vor allem auch in der Männerforschung immer wieder Irritationen hervor. Zum einen ist auf überraschende Weise das Subjekt immer noch sehr stark an das Individuum gekoppelt, auch wenn Subjektivität als Effekt diskursiver Strukturen gedacht wird. Nancy Fraser (vgl. 2001, 313) hat auch auf diesen Umstand aufmerksam gemacht und das Ausblenden von Intersubjektivität moniert. Die Intersubjektivität betrifft auch ein zweites Unbehagen mit poststrukturalistischen Identitätskonzeptionen, nämlich die Behauptung, dass nicht nur gender sondern auch sex konstruiert ist und dass die Konstruktionen theoretisch nicht an den Körper gebunden sind; vielmehr ist dieser selbst konstruiert. Barbara Duden (1993) hat Butler die Entkörperlichung der Frau vorgeworfen. Sie insistiert auf einer sinnlichen Dimension der Kategorie Geschlecht: „Stimme hat für mein Ohr immer Klang. Aus der Stimme klingt immer ein Mann oder eine Frau: beim ,ich'-Sagen braucht keine Stimme das Geschlecht anzugeben, denn es klingt aus dem gesprochenen ,ich'. Deshalb erschrecke ich, wenn hier ein stimmloser, stummer Diskurs, also reiner Text 
zur Grundlage des Wissens über Frauen gemacht wird“ (Duden 1993, 26). Nun ist der Klang der Stimme selbstverständlich auf die gleiche Weise diskursiviert, wie sex und gender. Dudens Argument kann Butlers Argumentation nicht aushebeln, es bestätigt vielmehr, dass man im Sprechen als Geschlechtswesen sichtbar und damit Subjekt wird. Worauf aber Duden aufmerksam machen will, ist, dass man doch immer schon als Geschlechtswesen ausgewiesen, markiert oder angerufen ist. Oder anders: Es erscheint, als sei Geschlecht immer schon gegeben, eine Gabe also, weshalb Kafka (1994) am 18. Januar 1922 in sein Tagebuch notieren kann: „Was hast Du mit dem Geschenk des Geschlechtes getan? Es ist misslungen, wird man schließlich sagen, das wird alles sein. Aber es hätte leicht gelingen können." Auch Butler (1991, 23) kommt auf das „gegebene“ Geschlecht zu sprechen: „Können wir noch von einem ,gegebenen' Geschlecht oder von einer ,gegebenen“ Geschlechtsidentität sprechen, ohne wenigstens zu untersuchen, wie, d.h. durch welche Mittel, das Geschlecht und/oder die Geschlechtsidentität gegeben sind?" Während sich also Butler für die Konstruktionsweisen interessiert, die zum „gegebenen“ Geschlecht führen, möchte ich die Perspektive forcieren, was aus der Gabe des Geschlechts folgt. Dudens Beispiel ist nicht so sehr ein Beispiel für ihre eigene Argumentation, sondern ist vielmehr, wenn auch hinter ihrem Rücken, ein schönes Beispiel für ein intersubjektives Setting, durch das Subjektivität entsteht, und zwar auf doppelte Weise: Im Sprechen tritt man als Subjekt in Erscheinung. Zweitens aber ist die Anrufung ein wesentlicher Aspekt von Intersubjektivität. Für eine männlichkeitskritische Geschlechterpolitik erweist sich womöglich die Gabe oder die Anrufung als vielversprechender denn die Analyse der Praxen der Naturalisierung von Geschlecht. Ich möchte mich dabei in aller Kürze auf zwei Konzeptionen beziehen, auf die Interpellation von Althusser und die Gabe von Derrida.

Interpellation: Althussers $(1977,133)$ berühmter Satz über Ideologie lautet: „Die Ideologie repräsentiert das imaginäre Verhältnis der Individuen zu ihren realen Existenzbedingungen.“ Wenn Männlichkeit als Ideologie funktioniert, dann bezeichnet Männlichkeit ein bestimmtes Verhältnis von Individuen zu ihren realen Existenzbedingungen. Folglich definiert sich Männlichkeit nicht über ihre Relationalität zu Weiblichkeit und umgekehrt, denn diese wechselseitige Definition ist tautologisch und bringt keinen Erkenntnisgewinn. Männlichkeit und Weiblichkeit nehmen nicht durch gegenseitiges Verweisen Bedeutung an, sondern dadurch, dass sie unterschiedliche Verhältnisse zu Existenzbedingungen bezeichnen. Männlichkeit bezeichnet also eine Verknüpfungsregel. Althusser nennt dieses Verhältnis „Anrufung“/,,interpellation“. „Die Ideologie ruft die Individuen als Subjekte an“ (ebda., S. 140). Männ- 
lichkeit bezeichnet erstens den Akt, durch den ein Individuum zu einem Subjekt wird. Daraus geht zweitens nicht nur hervor, dass das ,ideologische Subjekt" eine Tautologie ist, sondern dass Männlichkeit eine Position und eine Operation bezeichnet. Und Männlichkeit bezeichnet drittens dasjenige Prinzip, das die Operation bewerkstelligt und Geschlechterpositionen produziert. Während die beiden ersten Teile der Definition von Männlichkeit auf Weiblichkeit übertragbar sind, schließt das dritte Merkmal von Männlichkeit, das Grosz Phallozentrismus nennt, Weiblichkeit kategorisch als relationale Kategorie aus, weil es nicht zwei strukturierende Prinzipien für die Herstellung eines bedeutungsvollen Rahmens geben kann. Die Herrschaft des heterosexuellen Systems mit Oppositionsbildungen, die durch Dominanz miteinander verbunden bzw. voneinander getrennt sind, verdankt sich einem strukturbildenden Zentrum. Der Effekt, der von diesem Prinzip für das Verhältnis der Geschlechter ausgeht, nämlich reale männliche Dominanz, rechtfertigt es, von Phallozentrismus, also von einem männlichen Prinzip zu sprechen. Praktiken, in denen Individuen als Subjekte auftauchen, sind auch jene Praktiken, in denen Individuen bestimmte Geschlechterpositionen einnehmen. Diese Positionierungen sind vorläufig, unabgeschlossen, aufgeschoben und sie sind eine „,ideologische Fantasie“: „(T)hey know that, in their activity, they are following an illusion, but still, they are doing it" (Žižek 1994, 33). Dass Gendering eine niemals abschließbare ideologische Fantasie darstellt, heißt nicht, dass sie keine materielle Existenz hat. Materiell ist eine Ideologie deswegen, weil sie nie für sich selbst vorkommt, sondern sich in ideologischen Staatsapparaten und ihren Praktiken realisiert. Deswegen sagt Althusser (1977, 137): „Eine Ideologie existiert immer in einem Apparat und dessen Praxis oder dessen Praxen. Diese Existenz ist materiell.“ Und zwar ,insofern seine Ideen seine materiellen Handlungen sind, die in materielle Praxen eingegliedert und durch materielle Rituale geregelt sind, die ihrerseits durch den materiellen ideologischen Apparat definiert werden, dem die Ideen dieses Subjekts entstammen" (ebda., 139): Das heißt auch, dass Ideen keine ideale, geistige Existenz haben, sondern eine Existenz nur in konkreten Handlungen.

Die Gabe des Geschlechts: Poststrukturalistische Identitätskonzeptionen heften auf überraschende Weise Identität immer noch sehr stark an das Individuum, selbst dann, wenn Subjektivität als Effekt diskursiver Strukturen gedacht wird. Deswegen soll hier der Versuch unternommen werden, Gender an eine andere als individualistische Logik zu binden, und die These lautet, dass Geschlecht/Gender nach dem Prinzip von Ökonomie und Tauschlogik funktioniert. Ein Geschlecht im Sinne von Gender hat man nicht. Gender ist man auch nicht. Gender zirkuliert. Gender wird erworben, man bekommt es aufge- 
bürdet, es wird eingesetzt, getauscht und gegeneinander aufgerechnet. Nach dieser Logik kann Gender auch ein - willkommenes oder unwillkommenes Geschenk sein; ein Geschenk, dessen Annahme man zurückweisen oder das man annehmen oder zurückweisen kann oder vielleicht auch annehmen muss; eine Leihgabe, aber auch ein Auftrag. Geschlecht als Gabe erzeugt eine Verpflichtung, eine Verantwortung oder auch eine Schuld. Geschlecht als Gabe kann gewonnen und verspielt werden. Es gibt eine Vergeudung und Verschwendung von Geschlecht. Das Geschlecht als Gabe zu denken, heißt, das Geschlecht in einen Kreislauf der Ökonomie eingefügt zu sehen. „Die Gabe jedoch, wenn es sie gibt, bezöge sich ohne Zweifel auf die Ökonomie. Man kann nicht von der Gabe handeln, ohne von diesem Bezug auf die Ökonomie oder das Geld zu handeln, das versteht sich von selbst“(Derrida 1993, 16f.).

Aber die Gabe geht im Tausch nicht auf: „Aber ist die Gabe, wenn es sie gibt, nicht auch gerade das, was die Ökonomie unterbricht? Gerade das, was dem Tausch nicht mehr stattgibt, weil es den ökonomischen Kalkül suspendiert? Gerade das, was den Kreis öffnet, um sich der Reziprozität oder der Symmetrie, dem gemeinsamen Maß entgegenzustellen und so die Rückkehr in Rückkehrlosigkeit zu verkehren? Wenn es Gabe gibt, darf das Gegebene der Gabe (akkusativisch das, was man gibt, nominativisch das, was gegeben ist, die Gabe als das gegebene Ding oder als der Schenkungsakt) nicht zu dem Gebenden zurückkehren (sagen wir noch nicht zu dem Subjekt, zum Geber oder zur Geberin). Die Gabe darf nicht zirkulieren, sie darf nicht getauscht werden, auf gar keinen Fall darf sie sich, als Gabe, verschleißen lassen im Prozess des Tausches, in der kreisförmigen Zirkulationsbewegung einer Rückkehr zum Ausgangspunkt. Wenn die Figur des Kreises für die Ökonomie wesentlich ist, muss die Gabe anökonomisch bleiben. Nicht dass sie dem Kreis völlig fremd bliebe, aber sie muss dem Kreis gegenüber einen Bezug von Fremdheit bewahren, einen bezuglosen Bezug vertrauter Fremdheit. Und in diesem Sinne vielleicht ist die Gabe das Unmögliche“ (Derrida 1993, 17).

Die Gabe erlaubt es, das wäre eine provisorische These, mögliche Ausgänge für heterosexuelle Männlichkeiten aus dieser Tauschlogik zu denken und Geschlecht nicht mehr an Identitätslogiken zu binden, sondern dieses strikt ökonomisch zu denken. Geschlecht funktioniert dann wie das Geld bei Simmel. Es symbolisiert Wert, es macht sichtbar, man wird positioniert. Geschlecht wird ein Machtfaktor, der Kräfteverhältnisse bestimmt und Körper durchzieht. Die Formulierung von Geschlecht als Gabe im Anschluss an Derrida ist keine dritte oder vierte Lesart, eine völlig neue Position, die gegen alle anderen steht. Gerade dies wäre nicht die Position der Dekonstruktion. 


\section{Otherness, Produktivität und Räumlichkeit - Identität und Handlungsfähigkeit}

Grossbergs Kritik an Identitätskonzeptionen speist sich aus dem beschriebenen Unbehagen an einer individualistisch verkürzten Auffassung von Identität. Er weitet deshalb die kritische Analyse von Identität auf ihre modernen Grundlagen aus: auf Differenz, Individualität und Zeitlichkeit. Ihnen setzt er entgegen: Logiken der Otherness, der Produktivität und Rämlichkeit.

- Otherness: Die Logik des Anderen beruht auf der Annahme, dass der Andere unabhängig von einer spezifischen Beziehung zu dem Eigenen existiert und seinen eigenen Platz hat. Der Andere ist nicht mehr über eine Differenzrelation mit dem Eigenen verknüpft. Das bedeutet nun nicht, dass der Andere in essentialistischen Begriffen neu gefasst wird, sondern ,what it is can be defined by its particular (contextual) power to affect and be affected. That is, such view of otherness grant to each term an unspecified, but specifiable, positivity“ (Grossberg 1996a, 94). ${ }^{11}$ Affektive Logiken und Beziehungen bilden den Kern eines vom Eigenen unabhängigen Anderen. Bei Grossberg (vgl. 2000, 62) umfassen die Affekte Willenskraft, Stimmung, Beteiligung und Stärkung, die er vom Begehren und bloßen Vergnügen radikal trennt. Grossberg hat diese Unterscheidung in Bezug auf die Populärkultur getroffen und den Affekt als „Plateau der Effektivität“, ,die „Fähigkeit effektiv zu berühren und berührt zu werden“ (ebda.) bezeichnet. Somit wird Affekt zur Möglichkeit von Handlungsfähigkeit. Produktivität: Zur Logik der Identität gehört auch noch in der Version der radikalen Konstruktion - die Individualität. So formt der männliche Habitus die Männer und graviert sich ihrem Körper ein. Ob nun von einem authentischen Kern die Rede ist oder von der fragmentierten Identität, sie hat ihren Sitz im Individuum, das Identität, Bewusstsein und Handlungsfähigkeit artikuliert. Wenn nun Identität fragmentiert und unvollständig ist, stellt sich die Frage nach der Handlungsfähigkeit. Grossberg versucht nun, Handlungsfähigkeit von Identität zu lösen. Er fragt danach, was praktische Interventionen, die Realität transformieren, möglich

11 Grossberg verweist auf die rhizomatische Mannigfaltigkeit, die das Andere charakterisiert und den abstrakten Gegensatz zwischen Vielem und Einem vermeidet. Das Mannigfaltige, solchermaßen von jeder Dialektik gereinigt, organisiert keine Totalität mehr (vgl. Deleuze/Guattari 1997, 51). 
macht. Setzt Handlungsfähigkeit ein Subjekt voraus? Zumindest die strategische Supposition eines imaginierten ,vollständigen“ Subjekts? Diese Fragen werden durch das gemeinsame Modell der Repräsentation vereint, das die Handlungsfähigkeit als Repräsentation einer wie immer gefassten Identität versteht. Die Repräsentation ist umgekehrt mit einem Kausalmodell des Handelns verschweißt, das besagt, dass es für das Handeln Gründe gibt. Wenn man Handlungsfähigkeit allerdings nicht als Markierung des Subjekts begreift, sondern als konstituierendes Merkmal einer Zugehörigkeit, kurz als Produktion, dann löst sich Handlungsfähigkeit von Identität ab. Muss man als Mann entscheiden, ob man Feminist ist oder nicht? Ist es wichtig zu wissen (für andere oder mich selbst), ob die Unterstützung für Feministinnen an der Universität bloß strategisch ist oder aus - ja woraus eigentlich? - resultiert? Aus einer Überzeugung? Einer Ethik? Einem Wissen?

- Räumlichkeit: Der dritte Aspekt der Grossbergschen Dekonstruktion und Verschiebung der Identitätsproblematik betrifft die Logik der Zeitlichkeit, die er durch eine Logik des Räumlichen ersetzt. Hier geht es wesentlich um die Frage der Zugehörigkeit. Elspeth Probyn $(1995,53)$ zieht den Begriff belonging ${ }^{12}$ dem der Identität vor, weil es ihr so möglich wird, ,nicht in Standorten denken zu müssen, sondern mich an all den kleinen Linien der Sehnsucht zu orientieren“.

Auch Grossberg zielt nicht darauf ab, Identität aufzulösen und durch einen anderen Begriff zu ersetzen, sondern darauf, Handlungsfähigkeit und Identität von einander zu lösen und zu demonstrieren, dass sie nicht notwendig aufeinander rückführbar sind, ineinander übergehen und miteinander vernäht sind, sondern unterschiedlichen Logiken gehorchen und Bündnisse der Artikulation eingehen. „Social identities are themselves complex fields of multiple and even contradictory struggles; they are the product of the articulations of particular social positions into chains of equivalences, between experiences, interests, political struggles and cultural forms, and between different social positions“"(Grossberg 1996b, 156f.).

12 Probyn $(1995,54 \mathrm{f}$.) entfaltet das Bedeutungsspektrum von belonging: ,von ,jemandem oder einer Sache gehören' bis zu ,in die Gesellschaft passen“ oder ,Mitglied sein' geht es weiter zu belongings, was ,Besitztümer' und ,Gepäck' bedeuten kann. Für mich spiegelt belonging eine tiefsitzende Unsicherheit im Hinblick auf die Möglichkeit, wirklich dazuzugehören, wahrhaft in eine Umgebung zu passen, wider. Der Ausdruck belongings andererseits verweist auch auf die vielfältigen Arten, die es von dieser Sehnsucht, in eine Umgebung zu passen, gibt: freudvolle, schmerzliche, zum Scheitern verurteilte. Auf direktere Weise evoziert belonging Bilder des Weggehens an sich - es bezeichnet die Zwischenräume zwischen Sein und Sehnen, Kommen und Gehen.“ 


\section{Geschlechtsidentität und Männlichkeitskritik - Resümee}

Männlichkeitskritik positioniert sich zu Identität folgendermaßen: Nicht Identität, sondern Handlungsfähigkeit, Positionierung und Eingriff sind zentrale Begriffe für die Männerforschung. Wenn Handlungsfähigkeit mit Positionierung und Eingriff einhergeht, wenn Positionierung und Eingriff immer ein unbestimmtes $\mathrm{Ma} \beta$ an Verausgabung bedeuten, und zwar in der Form des Einsatzes von etwas, das nicht kontrollierbar ist, dann bedeutet es, dass Handlungsfähigkeit nicht in Relation zu Identität steht, sondern zu Ethik und Politik. Selbstverständlich hat Handlungsfähigkeit eine normative Grundlage, die sich aber in der Positionierung immer selbst überschreitet. In dieser Nichtkontrollierbarkeit der Überschreitung taucht auf, was Grossberg Otherness nennt.

Der Unterschied zu Judith Butler ist, auch wenn beide Konzeptionen antiessentialistisch konzipiert sind, eklatant. Das lässt sich am Beispiel des Wertes von Strategien sehen. Butler $(1991,20)$ betont, dass sich die Probleme der Repräsentation auch dann nicht verringern, ,wenn nur zu ,strategischen“ Zwecken an die Kategorie ,Frau(en) ${ }^{`}$ appelliert wird. Denn Strategien haben stets Bedeutungen, die über die angestrebten Ziele hinausgehen." Dieses Problem stellt sich nur dann, wenn man erstens die Frage der Handlungsfähigkeit an Identität und damit an Repräsentation bindet und zweitens, wenn man damit gleichzeitig unterstellt, dass es eine ,wahre', ,richtige' oder ,authentische ' Repräsentation für Praxen geben muss. Bei Butler hat ,strategisches Handeln" implizit etwas vom Vorwurf der Täuschungsabsicht, dem Spiel mit gezinkten Karten. Hier taucht noch einmal eine Form des „reinen“ Denkens auf. Das „reine“ Denken will sich als „starkes“ Denken auszeichnen, als ein Denken, das die unumstößliche Grundlage für Handlungen bildet. Unumstößlich heißt, es soll für das Handeln die zwingende Grundlage schaffen. Zwingend bezieht sich auf den Anderen, der dadurch das Handeln als ein zwingendes anerkennen soll. Strategie wird so zu einer Untugend der Täuschung, auch der Selbsttäuschung, der Schwäche des Geistes, zur Manipulation des Anderen, zur Vortäuschung falscher Absichten. Das starke Denken hingegen appelliert an eine bestimmte Logik von Objektivität, die an eine starke Subjektposition gebunden ist, der Haraway $(1995,86)$ 'ihren Objektivitätsbegriff entgegenstellt: „Das Versprechen der Objektivität liegt darin, dass wissenschaftlich Erkennende nicht die Subjektposition der Identität suchen, sondern die der Objektivität, das heißt der partialen Verbindung. Es gibt keine Möglichkeit, an allen Positionen zugleich oder zur Gänze an einer einzigen, privilegierten (unterdrückten) Position zu ,sein', die durch Geschlecht, ethnische und nationale Zugehörigkeit und Klasse strukturiert wird." 
Haraways „Vision“ findet ihren Abdruck auch in Drucilla Cornells (1991, 131) „ethical feminism“, ,[that] 'envisions' not only a world in which the viewpoint of the feminine is appreciated; ethical feminism also 'sees' a world 'peopled' by individuals, 'sexed' differently, a world beyond castration. Through our 'visions' we affirm the 'should be' of a different way of being human. The 'goal' of ethical feminism, which 'sees' the 'should be' inherent in the feminine viewpoint, is not just power for women, but the redefintion of all of our fundamental concepts, including power.“

Singularität ist für Grossberg (vgl. 1996a, 103) im Anschluss an Deleuze und Guattari und an Agamben ein Begriff, der Zugehörigkeit ohne Identität bezeichnet. Singularität ist eine Existenzweise, die weder universal noch partikular gedacht werden kann. Das Beispiel als Beispiel wäre für Agamben eine solche Existenzweise. Das Beispiel ist ein Zwischending, nicht bloß zufällig, aber auch nicht das Wesentliche, der Kern. Beispielhafte Praxis wäre eine werdende Praxis, die sich aber niemals ganz erfüllt.

\section{Männlichkeitskritik ist Patriarchatsanalyse}

Sexismus ist Sexismus erst durch eine zweite Form der Unterdrückung. Grosz nennt das dem Sexismus zugrunde liegende System „,patriarchal“. „Oberhalb und jenseits partikularer, konkreter sexistischer Handlungen liegt eine Struktur, die systematisch Männlichkeit in positiven und Weiblichkeit in negativen Termini wertet. Patriarchalismus ist damit ein struktureller Modus der Sozialorganisation, der Männer und Frauen in gesellschaftlichen, ökonomischen und interpersonellen Verhältnissen unterschiedlich positioniert. Er besteht nicht aus empirischen Handlungen; es ist eine latente Struktur, die diese individuellen Akte ermöglicht und sie in einer systematischen Form organisiert und den praktischen Kontext sowie die Bedeutungszusammenhänge sexistischer Ungleichheit liefert. Selbst wenn alle empirischen Beispiele für Sexismus wegfallen würden, würde die positionelle Unterdrückung von Frauen unverändert bleiben. Sie besteht nicht nur aus der unterschiedlichen und ungleichen Behandlung der beiden Geschlechter, sondern auch aus den differenten Bedeutungen und Wertungen, die ihnen zugeschrieben werden, auch wenn sie sich auf gleiche Weise verhalten. Was bei Männern als Zeichen von Stärke gewertet wird, würde bei Frauen als Aggressivität betrachtet werden, selbst bei identischem Verhalten. [...] Patriarchale Strukturen sind nicht unwandelbar, sondern historisch variabel und funktionieren in soziographisch spezifischen Kulturen auch auf spezifische Weise; dennoch behalten sie stets die 
Bestimmung, männliche Suprematie aufrechtzuerhalten und fortzuführen“ (Grosz, zit. nach Knapp 1993, 311f.). Als soziale Strukturkategorie ${ }^{13}$ existiert Geschlecht nur, wenn gesellschaftliche Macht entlang von Personengruppen mit klar definierten Gruppeneigenschaften (in diesem Fall Geschlecht) organisiert ist. Die Frage ist also nicht, ob es das Patriarchat gibt oder nicht, sondern wie das Patriarchat gedacht werden muss, um einerseits Sexismus zu ermöglichen, andererseits die vielfältigen Machtverhältnisse zwischen Männern und Frauen und innerhalb von Männern und Frauen zu repräsentieren. ${ }^{14}$

Es geht also um eine Theorie, die die Beweglichkeit des Patriarchats ausdrückt. Mann und Männlichkeit sind unzulängliche Verkürzungen, die den trügerischen Schein einer kollektiven Identität erzeugen. Aber löst die Vervielfältigung der Positionen dieses Dilemma? Repräsentieren viele Männlichkeiten eine Kategorie, die plausibel macht, dass Frauen nach wie vor in vielen Lebensbereichen diskriminiert werden? Muss die Analyse von Männlichkeit nicht bei dem Widerspruch einsetzen, dass den vielen widerstreitenden Männerinteressen ein institutionalisierter hegemonialer patriarchaler Apparat in die Hände arbeitet? ${ }^{15}$ Sind die vielfältigen männlichen Identitätsverhandlungen in einen phallozentrischen Rahmen eingebettet, der in dem Maße wirkt, wie er unsichtbar bleibt? Ist das Allgemeine männlich, unabhängig davon, wie widersprüchlich Männer untereinander handeln? So sehr auf der einen Seite herkömmliche theoretische Unterscheidungen und Kategorien dekonstruiert werden, so kommt man andererseits nicht umhin zu sehen, dass der Alltag immer noch von traditionellen Geschlechterrollenverteilungen geprägt wird.

13 Geschlecht als (soziale) Strukturkategorie ist Becker-Schmidt (vgl. 1993) zufolge eine von sieben Komponenten des Terminus' Geschlecht.

14 Genau so wie Butler das Subjekt des Feminismus, die Kategorie ,Frau(en)' kritisiert, kritisiert sie auch die Struktur des Patriarchats: „Die politische Annahme, dass der Feminismus eine universale Grundlage haben müsse, die in einer quer durch die Kulturen existierenden Identität zu finden sei geht häufig mit der Vorstellung einher, dass die Unterdrückung der Frauen eine einzigartige Form besitzt, die in der universalen oder hegemonialen Struktur des Patriarchats bzw. der männlichen Herrschaft auszumachen sei. Allerdings ist die Vorstellung von einem universalen Patriarchat in den letzten Jahren auf breite Kritik gestoßen, weil sie unfähig ist, den spezifischen Vorgehensweisen der Geschlechterunterdrückung in den konkreten kulturellen Zusammenhängen Rechnung zu tragen“ (Butler 1991, 18f.).

15 Einen Beitrag, auf den ich hier nicht näher eingehen kann, liefert Iris Marion Young, die im Anschluss an Sartres Unterscheidung zwischen Gruppe und Serie die Problematik dessen, was eine Gruppe definiert, aufzulösen versucht und damit ein zentrales Thema feministischer Politik angeht: „Einerseits verliert eine feministische Politik ihre Besonderheit, wenn sie nicht in irgendeiner Weise davon ausgeht, daß es ein gesellschaftliches Kollektiv 'Frauen' gibt. Andererseits untergräbt aber anscheinend jeder Versuch, die Eigenschaften dieses Kollektivs zu definieren, die feministische Politik, weil dadurch notwendig manche Frauen ausgeschlossen werden, die der Feminismus einbeziehen müsste“(Young 1994, 24). 


\section{Patriarchatsdividende}

Robert Connell (1999) nennt die männliche Teilhabe am Patriarchat „Patriarchatsdividende" um auszudrücken, dass wir Männer, ob wir wollen oder nicht, in privilegierte Positionen gesetzt sind. Es gibt weder den unschuldigen, das heißt geschlechtsneutralen Blick, noch ein solches Handeln. Was immer ein Mann sagt und tut, in einem patriarchalen Kontext wird es als geschlechtliches Handeln bedeutsam: Es kann als typisch männliches Handeln wahrgenommen werden oder auch als irritierendes Handeln, da es den unterstellten Normen nicht entspricht, etc. Aber es wäre eine Illusion zu glauben, dass es ein Sprechen vor der sexuellen Markierung gibt. Unklar bleibt, auf welche Weise unterschiedliche Formen der Teilhabe zustande kommen. Und es hat den Anschein, als habe die Veränderung von Männlichkeitsbildern und männlichen Handelns in den vergangenen Jahrzehnten wenig an der Wirkkraft des Patriarchats verändert, so als funktioniere dieses wie ein Selbstläufer unterhalb der sich rasch ändernden Geschlechterpraktiken an der Oberfläche der Gesellschaft. ${ }^{16}$

\section{Die Beweglichkeit des Patriarchats}

Um die Funktionsweise des Patriarchats zu verstehen, ist es wichtig, seine vereinheitlichende Funktion und seine verkettende und substituierende Beweglichkeit zu verstehen. ${ }^{17}$ Darin gleicht das Patriarchat der Funktionsweise des Rassismus. Die vereinheitlichende Funktion des Rassismus besteht in seiner Ausgrenzung und sozialen Normalisierung, seine Beweglichkeit aber darin, dass er seine Funktion auf allen gesellschaftlichen Feldern durchsetzt und so an Stärke und Legitimation gewinnt. Soziale Identitäten sind jene komplexen Schauplätze, die die Struktur für die Beweglichkeit des Rassismus bilden, denn auf ihnen finden vielfache und widersprüchliche Kämpfe statt, bei denen

16 Ein zweites Modell, um die Frage männlicher Teilhabe am Patriarchat zu lösen, ist das Habitus-Konzept von Bourdieu, auf das sich so unterschiedliche Theoretiker wie Ralf Bohnsack (2001), Lothar Böhnisch (2001), Holger Brandes (2002) und Michael Meuser (1998) beziehen. Parallelen zu Connells Patriarchatskonzept sind unübersehbar, auch wenn Connell Bourdieus Habitus-Konzept als zu funktionalistisch zurückweist (vgl. Brandes 2002, 61, Anm. 3). Ich kann hier nicht näher auf das Habitus-Konzept und seine Probleme eingehen (vgl. Forster/Rieger-Ladich 2004).

17 Auf ähnliche Weise habe ich versucht, das Problem des Rassismus und der Rassismusprävention anzugehen (vgl. Forster 2001). 
unterschiedliche Differenzen miteinander verhandelt werden. In diesen Identitätskämpfen verknüpft sich der Rassismus mit dem Nationalismus, mit dem Plädoyer für kulturelle Differenz, mit Argumenten zur Sprachgemeinschaft, mit Wirtschaftslogik, gleichermaßen mit Sexismus und Antisexismus, mit Generationenkonflikten usw.

Die Beweglichkeit des Patriarchats besteht in seiner mühelosen Artikulationsfähigkeit. Die Theorie der Artikulation beschreibt die kontingente (nichtkausale) Verknüpfung machtvoller kontextgebundener und kontextproduzierender ethnischer, Generationen-, Geschlechter- und Klassen-Differenzen (vgl. Hall 2000). Artikulation heißt Verbindung und Substitution einzelner Elemente einer Kette. Laclau (1997) nennt sie Äquivalenzkette. Auf diese Weise werden in alltäglichen Praktiken soziale Identitäten (re-)produziert und (re-)präsentiert. Die Konstruktion des ,Wir' und der Anderen wird so zu einer beständigen Re-Artikulation machtvoller Inklusions- und Exklusionsprozeduren.

Prävention gegen Exklusion und soziale Normierung operieren gegen die Verkettungslogik und seine Mythenproduktion. Das Geschlechterverhältnis ist ein Feld, auf dem Exklusion und soziale Normierung besonders reibungslos funktionieren, weil hegemoniale Männlichkeit und die sie stützenden Strukturen vielfach unsichtbar bleiben.

\section{Männlichkeit und Äquivalenzlogik}

Wenn man Männlichkeit als Ideologie begreift, dann gelingt es, so meine These, ein nicht-essentialistisches Männlichkeitskonzept mit struktureller männlicher Dominanz zusammen zu denken. Wenn man Männlichkeit als Ideologie versteht, weist man die Behauptung eines außerdiskursiven Standpunktes zurück. Zwar wird der Anschein erweckt, dass es eine volle Bedeutung von Männlichkeit gibt, tatsächlich aber wird diese Bedeutung durch eine „fiktive Fixierung" hergestellt, die Männlichkeit notwendig als falsche Repräsentation installiert. Die Illusion von der Wahrheit des Geschlechts verschleiert die Tatsache, dass die fiktive Fixierung über Inklusions- und Exklusionsmechanismen gewaltsam hergestellt wird. Männlichkeit als Ideologie zu begreifen, ist der Versuch, Männlichkeit als Funktionsweise bzw. als Operation zu fassen. Zusammengefasst lässt sich Männlichkeit folgendermaßen beschreiben: 
○ Männlichkeit wird durch Praktiken produziert, verändert und repräsentiert.

- Mit bedeutsamen Praktiken situiert sich ein Individuum als Subjekt in einem Diskurs.

- Die historisch konstituierten Bedeutungen von Praktiken sind geschlechtlich, ethnisch etc. kodiert, sodass man in diesem Diskurs immer nur und zugleich als Geschlechts-, Klassen-Subjekt etc. auftaucht.

○ Männlichkeit wird durch eine Kette von Bedeutungen - Laclau nennt sie Äquivalenzkette - repräsentiert. Keine Bedeutung ist imstande, Männlichkeit als Ganzes zu fassen.

○ Ideologisch ist jede Bedeutungsfixierung, denn jede volle Bedeutung ist disloziert. In der falschen Repräsentation besteht die der Ideologie immanenten Idee der Verzerrung.

Indem Männlichkeit Bedeutungen fixiert, produziert sie Differenzen, die wiederum Macht organisieren. Männlichkeit unterscheidet sich von anderen Geschlechterpositionen durch die Art und Weise der Differenzproduktion. Männlichkeit funktioniert als Ideologie, weil Männlichkeit etwas anderes bedeutet als sie vorgibt zu sein: Essenz, Substanz, Eigenschaft. Mit Männlichkeit verbindet sich die Vorstellung eines Erkenntniszuwachses über Identität, während sie tatsächlich das Scheitern dieser Erkenntnis repräsentiert (vgl. Copjec 1995).

Ideologie lässt sich auf drei Achsen situieren: Erstens ist Ideologie ein Gefüge von Ideen: Theorien, Überzeugungen etc. Zweitens ist sie die Materialität dieser Ideen: ideologische Staatsapparate (religiöse Institutionen, Familie etc.). Drittens ist Ideologie „, ,spontane“ Ideologie, die im Herzen der gesellschaftlichen ,Realität‘ selbst am Werk ist“" (Žižek, zit. nach Laclau 1997, 45). „Spontane Ideologie“ realisiert sich auf der Ebene alltäglicher Praktiken und gesellschaftlicher Institutionen. Als „legitimierte Situationsdefinitionen“ (Falk/Steinert 1973, 39) produzieren Institutionen Regeln für Zugehörigkeit. Sie funktionieren immer über Inklusion und Exklusion (vgl. Douglas 1991).

Auf diese Weise ergibt sich eine Definition von Männlichkeit durch das Zusammenspiel von drei Dimensionen: Männlichkeit definiert sich durch alltägliche Praktiken, deren Bedeutungen durch diskursive Rahmenbedingungen hergestellt und verändert werden und sich in institutionellen Materialisierungen niederschlagen. Diese Dreiteilung findet sich in Grosz' Analyse von Machtverhältnissen wieder: Sexismus, Patriarchalismus und Phallozentrismus. Sexismus drückt Männlichkeit in Form konkreter, alltäglicher Praktiken aus. Patriarchalismus unterstützt sexistische Praktiken durch Institutionen. 
Phallozentrismus bezeichnet die legitimierte Autorität, die Regeln vorgibt, nach denen Institutionen und Praktiken funktionieren und ihre Bedeutung im (Geschlechter-)Diskurs annehmen. Daraus folgt, dass Männlichkeit nicht nur ein Element in der Geschlechterdifferenz ist, sondern das Strukturierungsprinzip, das die Differenz bestimmt.

Das Argument, Männlichkeit funktioniere als Ideologie, enthält zwei Annahmen: Erstens macht das Reden von Ideologie nur Sinn, wenn es einen Bereich des Nichtideologischen gibt. Zweitens: Der Begriff Ideologie enthält die Idee der Verzerrung in Gestalt der Unwahrheit oder Täuschung. Die sex-gender-Trennung hat es ermöglicht, sex als außerdiskursiven Fixationspunkt aufrechtzuerhalten. Dass eben darin die Ideologie der Geschlechterkonzeption bestand, hat Judith Butler $(1991,26)$ nachgewiesen. Es geht in der Folge darum zu sehen, dass die Dominanz des Männlichen durch den Anschein erzeugt wird, es handle sich um Naturwüchsigkeiten, die deswegen als solche nicht wahrgenommen werden. Diese ideologische Täuschung unterscheidet Männlichkeit grundlegend von Weiblichkeit.

Die Annahme, dass die Idee eines außerdiskursiven Standpunktes „die ideologische Täuschung schlechthin“ (Laclau 1997, 48) sei, führt Laclau zu folgender Frage: Wie kann man an einem Begriff der Verzerrung und damit am Begriff der Ideologie festhalten, ohne den für den Begriff der Verzerrung notwendig außerdiskursiven Standpunkt einnehmen zu müssen? Nicht nur das Außerdiskursive, sondern auch das Diskursive ist Ideologie, denn das Soziale muss als symbolisches System vorgestellt werden, als Netz von Signifikanten, unter die sich frei flottierende Bedeutungen schieben. Will man Bedeutungen fixieren, muss dieses System zum Stillstand gebracht werden, indem man einem Signifikanten genau ein Signifikat zuordnet. Diese Fixierung nennt Laclau Schließung. Sie ist notwendig ideologisch.

Die Verzerrungsoperation besteht darin glauben zu machen, es gäbe eine ursprüngliche, unverzerrte Bedeutung. Die Verzerrung besteht darin, dass auf etwas „fundamental Gespaltenes die Illusion einer Fülle und Selbsttransparenz" (ebd., 50) projiziert wird. Männlichkeit produziert die Illusion einer vollen Bedeutung von Männlichkeit. Zur Verzerrung gehören die Dislokation der vollen Bedeutung und die Verschleierung der Dislokation. Damit es Bedeutung gibt, braucht es eine „fiktive Fixierung“ (ebda., 51). Sie ist „falsche“ Repräsentation, das, „woraus jede Identität ihre fiktive Kohärenz zieht“. Männlichkeit gibt auf diese Weise der geschlechtlichen Identität eine fiktive Kohärenz. Da die Schließung notwendig aber unmöglich ist, hat sie keinen eigenen Inhalt. Sie ,zeigt sich lediglich durch ihre Projektion auf ein von ihr verschiedenes Objekt" (ebda., 52). Zwischen diesem Objekt und der SchlieBungsoperation gibt es eine wechselseitige Abhängigkeit. Ideologisch werden 
Praktiken, wenn diese etwas über sie Hinausgehendes und von ihnen selbst Verschiedenes zu inkarnieren beginnen. Damit Ideen mehr als diese sind und Ideologien werden, muss es eine „Horizont-Dimension“ geben, innerhalb derer einzelne Praktiken als Elemente fungieren und Bedeutung durch ihre Opposition gegenüber allen anderen Elementen annehmen. Eine Vielzahl unterschiedlicher Praktiken können ein äquivalenter Name für Männlichkeit abgeben: ... $\leftrightarrow$ physische Stärke $\leftrightarrow$ Herrschaft $\leftrightarrow$ Dominanz $\leftrightarrow$ Gewalt $\leftrightarrow$ Durchsetzungsvermögen $\leftrightarrow$ Erfolg $\leftrightarrow$ Karriere $\leftrightarrow$ Geld $\leftrightarrow$ Heterosexualität $\leftrightarrow$... Ist Männlichkeit nun ein flottierender Signifikant, der überdeterminiert ist und Sinnüberschuss produziert, oder ist er ein leerer Signifikant, ein Signifikant ohne Signifikat? Laclau $(1997,55)$ zufolge ist der „flottierende Charakter eines Signifikanten die einzige Erscheinungsform seiner Leere“. Männlichkeit ist ein flottierender Signifikant und deshalb imstande, eine endlose Äquivalenzkette zu erzeugen. Dabei werden ständig Oppositionen gebildet, so dass die Behauptung gerechtfertigt erscheint, dass Bedeutungsproduktionen auch über oppositionserzeugende Inklusions- und Exklusionsmechanismen funktionieren. Äquivalenzketten haben eine doppelte Ausdehnung: Elemente verknüpfen sich zu einer Kette. Gleichzeitig produzieren aber Elemente oppositionelle Elemente, die sich selbst zu einer Art „oppositioneller Kette" verknüpfen. Auf diese Weise werden über Äquivalenzketten und das heißt durch Identitätspraktiken permanent Grenzen produziert und verändert.

Hegemonie setzt die Logik des leeren Signifikanten voraus. Männlichkeit tendiert zur Hegemonie genau dann, wenn sie nicht nur männliche Identiät fixiert, sondern überhaupt Identität; wenn sie also auch Weiblichkeit und andere Männlichkeiten fixiert; wenn sie schließlich das Geschlechterverhältnis regelt; wenn sie, kurz gesagt, den Diskurs der Geschlechter fixiert und wenn sie darüber hinaus, jenseits des Geschlechterdiskurses, Normalität regelt., 18 Dabei muss aber berücksichtigt werden, dass der Hegemonie produzierende Prozess auch sein Gegenteil ermöglicht: Zerstreuung.

18 „Hegemonie ist eine Angelegenheit von Zustimmung, nicht von Konsens, und es ist eine besondere, historisch neue Form des Kampfes um Macht, weil sei vom Aufkommen ,der Massen“ als politisches und kulturelles Subjekt abhängt“ (Grossberg 2000, 58f.). 


\section{Männlichkeitskritik und ihr Verhältnis zu feministischen Theorien und Praxen}

Wenn man Modelle zur Jungen- und Männerarbeit sowie zur Männerforschung einer kritischen Reflexion unterzieht, dann stellt man rasch eine Bruchlinie fest, die besonders sensibel zu sein scheint und deshalb für die Analyse von Männlichkeitskritik zielführend und wertvoll ist. Diese Bruchlinie betrifft das Verhältnis von Männerarbeit und Männerforschung zur feministischen Theorie und Praxis, also zu jenem vielgestaltigen Diskurs, den (zumindest einige) Männer ab sofort mitbestimmen und mittragen, aber auch verändern und umdefinieren wollen. Leitbegriffe dieser Bruchlinie sind: Feminismus, Antisexismus und Patriarchatskritik.

Männlichkeitskritik heißt zuerst, ihr Verhältnis zum Feminismus zu befragen. Die anderen Bruchlinien und Problemfelder stehen damit in engem Zusammenhang (vgl. Forster 2002).

In der Einführung zum Buch Nur Macher und Macho? Geschlechtsreflektierende Jungen- und Männerarbeit von Kurt Möller (1997, 11) ist zu lesen: „Jungen- und Männerarbeit hegt nicht den Anspruch, Feministen heranzuziehen, ein Unterfangen, das ohnehin paradox wäre." Es ist erstaunlich festzustellen, dass eine Reihe von Ansätzen in der Jungen- und Männerarbeit mit einer Distanzierung zu feministischer Theorie und Praxis und mit einem Zurückweisen von Forderungen von Frauen beginnen. Der Begriff „Feminist“ erfährt - hier - eine ambivalente Wertung. Zumindest in Form der losen Assoziation taucht eine typisch homophobe Strategie auf: Jungen und Männer werden abgewertet, indem sie ,verweiblicht“" werden bzw. indem ihre Anliegen zu weiblichen Anliegen gemacht werden. Ähnliches soll wohl mit der Aussage von Kurt Möller suggeriert werden: dass es paradox sei, wenn Jungen eine feministische Haltung einnehmen. ${ }^{19}$

Männlichkeitskritik verdankt feministischen Theorien und Praxen entscheidende Impulse. Gemeinsam entwickeln sie Strategien für die Durchsetzung einer umfassenden Geschlechterdemokratie - und folgen dabei selbst einem Demokratiekonzept, das Chantal Mouffe (vgl. 1997) formuliert hat:

19 Frauen, die sich für Frauenrechte einsetzten, und Männer, die Frauen darin unterstützten, wurden um die Jahrhundertwende abwertend „Feministen“" genannt. Ich zitiere aus dem Artikel Die Feministen, den der Freud-Schüler Fritz Wittels 1908 in der Fackel publiziert hat: „Ein sehr großer Teil der Feministen besteht aus Spießbürgern, die auf ein fortschrittliches Programm en bloc eingeschworen sind. Sie meinen, dass man alles emanzipieren müsse: die Juden, die Neger und also auch die Frauen.“ (12) Und an anderer Stelle: „Die unerbittlichsten Feministen endlich sind die, deren Liebe einer emanzipierten Frau gehört.“ (13) (Ich danke Andrea Bramberger für diesen Hinweis.) 
Männlichkeitskritik benötigt keine Grenzen zum Feminismus, keine „eigenen" Strategien, keine Selbständigkeit und Unabhängigkeit, sondern die Bereitschaft, sich auf ein Projekt einzulassen. Die ,wenigen' Grundregeln, die die Voraussetzung für (Geschlechter-)Demokratie bilden, betreffen nichts als die Bereitschaft, in der Verhandlung mit anderen die eigene Identität zu verändern. Die Grundlage für einen solchen Verhandlungsprozess ist freilich, dass diese Bereitschaft von allen gleichermaßen gegeben ist. Und das ist die Grundlage dafür, dass alle an der Verhandlung teilnehmen können, denn Exklusionen aus einem als offen konzipierten Projekt beruhen immer darauf, dass die Nichtausgeschlossenen ihre Identität (ihre Privilegien) eben nicht aufgeben wollen und damit aus einem zukünftigen Projekt eine gegenwärtige Einheit machen.

\section{Wider Resouveränisierung und Immunisierung}

Pierre Bourdieu beginnt seine Ausführungen über Die männliche Herrschaft mit einer erkenntnistheoretischen Betrachtung. Er verweist nicht nur darauf, dass Wissenschafter mit Vorurteilen und Vorannahmen an ihre Gegenstände herangehen, sondern dass sie Wahrnehmungs- und Denkkategorien als Erkenntnismittel verwenden, die sie als Erkenntnisgegenstände analysieren möchten (vgl. Bourdieu 1997a, 153ff.). Die Schwierigkeiten der Sozialwissenschaften bestehen darin, Instrumente zum Denken zu verwenden, die bereits konstruiert sind. „Alles, worüber wir zum Denken verfügen, ist bereits gendered, geschlechtsstrukturiert“ (Bourdieu 1997b, 221). Es geht aber nicht allein darum, die Erkenntnismittel auf ihre impliziten Voraussetzungen zu kontrollieren, denn das imaginiert noch einmal die Illusion, dass über die Kontrolle all der Vorannahmen eine ,reine' Analyse möglich sei, während jede Form des Theoretisierens unabhängig von den verwendeten Denk- und Wahrnehmungskategorien den Gegenstand der Erkenntnis im Sinne der Theorie der Performativität reinszeniert.

Ich habe an anderer Stelle zu zeigen versucht (vgl. Forster 2004), auf welche Weise die Unterschlagung des performativen Effekts in Robert Connells Entwurf einer akademischen Disziplin Kritische Männerforschung wirksam wird und die kritische Intention in ihr Gegenteil umschlägt. Die kritische ,Rede' von hegemonialer Männlichkeit und Patriarchatsdividende erzeugt nämlich einen eigentümlichen Effekt: Sie wird suspekt, weil sie mit der kritischen Position auch ein ,starkes', unangreifbares Subjekt erzeugt. Kritik er- 
scheint, so meine These, als Immunisierungsstrategie gegen die Möglichkeit von Kritik und als Resouveränisierungsstrategie des männlichen Subjekts. ${ }^{20}$

Mir geht es nicht darum, Connells kritisches Männlichkeitsprojekt einfach $\mathrm{zu}$ verwerfen. Es geht auch nicht um andere Fragen oder eine andere Theorie, mit der Männlichkeitsforschung angemessener betrieben werden könnte. Ich möchte vielmehr auf einen kaum wahrgenommenen Punkt insistieren, an dem Kritik kippt und sich in ihr Gegenteil verkehrt. Dieses Kippmoment entsteht durch Connells Strategie der Exklusion. Sie erzeugt ein mächtiges, wissendes Subjekt, das die Definitionsmacht über einen Gegenstand an sich gerissen hat. Dadurch bringt Connell eine fatale Koinzindenz hervor: Er inthronisiert sich als eben jenes männliche, wissende Subjekt, das er auf der Objektseite kritisiert. Weshalb taucht diese Reproduktion einer klassischen männlichen Position bei Connell nicht als Widerspruch auf? Connell ist seine eigene Rede nicht suspekt, weil er sie nicht als (seine) ,Rede', sondern als eine von ihm abgekoppelte Deskription über, Wirklichkeit" begreift. Mit anderen Worten: Er negiert die Performativität seines Textes, läßt sich als Autor-Ich verschwinden und verwandelt seine ,Rede' in eine Reihe konstativer Statements. Auf diese Weise entsteht eine kritische Männlichkeitsforschung im Rahmen eines Wissenschaftsverständnisses, das von feministischen Theoretikerinnen (vgl. z.B. Fox-Keller 1998, Haraway 1995, Harding 1998; 1994) immer wieder als männlich und/oder phallogozentrisch kritisiert worden ist.

Rituale der Kritik, der vernünftigen Rede und einer abstrakten Selbstreflexion zielen auf die Reproduktion eines - kritischen - Subjekts des Diskurses über Männerforschung. Sie alle machen das Subjekt unangreifbar. Wenn für hegemoniale Männlichkeit ihre Unsichtbarkeit charakteristisch ist, so wird diese von kritischer Männerforschung ans Licht gezerrt - um den Preis, dass sich nur der blinde Fleck verschiebt und nun in der Theorieproduktion wirksam ist.

20 Vgl. dazu auch die kritische Sammelrezension von Siegfried Kaltenecker (1998, 125): „Hier werden allerorts große Programme geschmiedet, feministische Defizite fokussiert und die Unumgänglichkeit eines männerspezifischen Blicks eingeklagt. Selbsternannte Kritiker gerieren sich als ,Saboteure' der Männerherrschaft, um gleichzeitig auf den ,eigenen Interessen als Männer` zu beharren und ,zuerst einmal für mich (zu) kämpfen‘.“ 


\section{Politische Strategien}

Dies verlangt nach einer doppelten politischen Strategie, die zunächst in einem kritischen Gestus besteht, der nicht zu einer Resouveränisierung oder Immunisierung von Männlichkeit führt und ein starkes männliches Subjekt des Genderdiskurses (re-)produziert. In der Praxis lassen sich Resouveränisierungs- oder Immunisierungsstrategien sehr leicht daran erkennen, wie Männerforschung und Männerarbeit ihr Verhältnis zu feministischen Theorien und Praxen definieren. Was heißt das praktisch?

○ Männlichkeitskritik übt radikale Wissenschaftskritik derart, wie etwa Haraway sie formuliert.

○ Männlichkeitskritik formiert keine neue Disziplin (vgl. dagegen Connell 2000, Forster 2004).

○ Keine Lehrstühle für Gender Studies an Männer; Männlichkeitskritik ist eine Praxis, die Männer von dort aus machen, wo sie immer schon sitzen.

○ Männlichkeitskritik heißt für MännerVerantwortung zu übernehmen für eine demokratische Geschlechterpolitik.

○ Männlichkeitskritik heißt: Sich einzumischen - wie? wozu?

Zum anderen müsste die politische Strategie lauten, offene Räume zu schaffen, die dazu animieren, in Verhandlungen zu treten oder, wie Donna Haraway (1990) es ausdrückt, ,partial connections“ zu bilden. In Verhandlungen $\mathrm{zu}$ treten heißt, mit anderen eine „Gruppe“ zu bilden. Gruppen werden nicht durch gegebene Gemeinsamkeiten, durch gegebene Identitäten bestimmt, sondern durch die Bereitschaft, solche zu (er)finden. Der Ausgangspunkt ist nicht das Gegebene, sondern eine (mögliche) Zukunft. Elspeth Probyns (1995) Begriff der „queer belongings“ bezeichnet diese Haltung: Sich nicht auf vorgegebene, hegemoniale Zugehörigkeiten zu verlassen, sondern in einen aktiven Prozess des (Er-)Findens einzutreten. Beziehungen dieser Qualität sind offen, kontextgebunden, flüchtig. Solche Formen der Gruppenkonstituierung zu verallgemeinern heißt, politische Gemeinschaften zu entwerfen, die sich nicht auf ein Geschlecht, eine Nation, eine Kultur, eine Ethnie, eine Generation berufen, sondern auf eine unabsehbare Zukunft. 


\section{Literatur}

Althusser, Louis (1977): Ideologie und ideologische Staatsapparate. Aufsätze zur marxistischen Theorie. Hamburg/Westberlin

BauSteineMänner (Hg.) (2001): Kritische Männerforschung. Neue Ansätze in der Geschlechtertheorie. Hamburg: Argument (3., erweiterte Aufl.)

Becker-Schmidt, Regina (1993): Geschlechterdifferenz - Geschlechterverhältnis: Soziale Dimensionen des Begriffs „Geschlecht“. In: Zeitschrift für Frauenforschung 11 , Heft $1 / 2$

Bly, Robert (1991): Eisenhans. Ein Buch über Männer, München

Brandes, Holger (2002): Der männliche Habitus. Band 2: Männerforschung und Männerpolitik, Opladen

Bieringer, Ingo/Buchacher, Walter/Forster, Edgar (Hg.) (2000): Männlichkeit und Gewalt. Konzepte für die Jungenarbeit, Opladen

Böhnisch, Lothar (2001): Männlichkeiten und Geschlechterbeziehungen - Ein männertheoretischer Durchgang. In: Margrit Brückner \& Lothar Böhnisch (Hg.): Geschlechterverhältnisse. Gesellschaftliche Konstruktionen und Perspektiven ihrer Veränderung, Weinheim \& München

Bohnsack, Ralf (2001): Der Habitus der „Ehre des Mannes“. Geschlechtsspezifische Erfahrungsräume bei Jugendlichen türkischer Herkunft. In: Döge, Peter \& Meuser, Michael (Hg.): Männlichkeit und soziale Ordnung. Neuere Beiträge zur Geschlechterforschung, Opladen

Bourdieu, Pierre (1997a): Die männliche Herrschaft. In: Dölling, Irene \& Krais, Beate (Hg.): Ein alltägliches Spiel. Geschlechterkonstruktion in der sozialen Praxis, Frankfurt a.M.

Bourdieu, Pierre (1997b): Eine sanfte Gewalt. Pierre Bourdieu im Gespräch mit Irene Dölling und Margareta Steinrücke. In: Dölling, Irene \& Krais, Beate (Hg.): Ein alltägliches Spiel. Geschlechterkonstruktion in der sozialen Praxis, Frankfurt a.M.

Butler, Judith (1991): Das Unbehagen der Geschlechter, Frankfurt a.M.

Connell, Robert W. (2000): The Men and the Boys. Berkeley \& Los Angeles: UC Press

Connell, Robert W. (1999): Der gemachte Mann. Konstruktion und Krise von Männlichkeiten, Opladen

Cornell, Drucilla (1991): Beyond Accomodation. Ethical Feminism, Deconstruction, and the Law. Lanham u.a.: Rowman \& Littlefield (New Edition).

Copjec, Joan (1995): Das Geschlecht und die Euthanasie der Vernunft. In: texte psychoanalyse. ästhetik. kulturkritik 15, H. 1

Deleuze, Gilles (1997): David Hume, Frankfurt a.M.

Deleuze, Gilles (1993): Logik des Sinns, Frankfurt a.M.

Deleuze, Gilles (1989): Zeichen und Ereignisse. Ein Gespräch mit François Ewald und Raymond Bellour. In: Pariser Gespräche, Berlin 
Deleuze, Gilles/Guattari, Félix (1997): Tausend Plateaus. Kapitalismus und Schizophrenie, Berlin

Derrida, J. (1998): Auslassungspunkte - Gespräche, Wien

Derrida, Jacques (1993): Falschgeld - Zeit geben I, München

Derrida, Jacques (1986): Positionen. Gespräche mit Henri Ronse, Julia Kristeva, JeanLouis Houdebine, Guy Scarpetta, Graz/Wien

Döge, Peter (2001): Geschlechterdemokratie als Männlichkeitskritik. Blockaden und Perspektiven einer Neugestaltung des Geschlechterverhältnisses, Bielefeld

Döge, Peter/Meuser, Michael (2001): Männlichkeit und soziale Ordnung. Neuere Beiträge zur Geschlechterforschung, Opladen

Douglas, Mary (1991): Wie Institutionen denken, Frankfurt a.M.

Duden, Barbara (1993): Die Frau ohne Unterleib: Zu Judith Butlers Entkörperung. Ein Zeitdokument. In: Feministische Studien, H. 2

Düttmann, A. G. (1992): Die Dehnbarkeit der Begriffe. Über Dekonstruktion, Kritik und Politik. In: Georg-Lauer, J. (Hg.): Postmoderne und Politik, Tübingen

Engelmann, Peter (1986): Positionen, 14 Jahre später. In: Derrida, Jacques: Positionen. Gespräche mit Henri Ronse, Julia Kristeva, Jean-Louis Houdebine, Guy Scarpetta, Graz/Wien

Falk, Gunter/Steinert, Heinz (1973): Über den Soziologen als Konstrukteur von Wirklichkeit, das Wesen der sozialen Realität, die Definition sozialer Situationen und die Strategien ihrer Bewältigung. In: Steinert, Heinz (Hg.): Symbolische Interaktion. Arbeiten zu einer reflexiven Soziologie, Stuttgart

Forster, Edgar (2004): Männlichkeitsrituale als Widerstandsrituale in Erziehung und Bildung. Zur Konstruktion des (kritischen) Subjekts im Männlichkeitsdiskurs. In: Zeitschrift für Erziehungswissenschaft, 6. Jg., Beiheft 2

Forster, Edgar (2002): Jungenarbeit als Männlichkeitskritik. In: Kofra - Zeitschrift für Feminismus und Arbeit, Heft 96

Forster, Edgar (2001): Die Beweglichkeit des Rassismus. In: TANGRAM - Bulletin der Eidgenössischen Kommission gegen Rassismus. Nr. 10

Forster, Edgar/Rieger-Ladich, Markus (2004): Männerforschung und Erziehungswissenschaft. In: Glaser, Edith/Klika, Dorle/Prengel, Annedore (Hg.): Handbuch Gender und Erziehungswissenschaft, Bad Heilbrunn

Foucault, Michel (1996): Der Mensch ist ein Erfahrungstier, Frankfurt a.M.

Fox-Keller, Evelyn (1998): Liebe, Macht und Erkenntnis. Männliche oder weibliche Wissenschaft? Frankfurt a.M.

Fraser, Nancy (2001): Die halbierte Gerechtigkeit. Schlüsselbegriffe des postindustriellen Sozialstaats, Frankfurt a.M.

Grossberg, Lawrence (2000): What's going on? Cultural Studies und Popularkultur, Wien

Grossberg, Lawrence (1996a): Identity and Cultural Studies - Is That All There Is? In: Stuart Hall and Paul duGay (Eds), Questions of Cultural Identity. London/Thousand Oaks/New Delhi 
Grossberg, Lawrence (1996b): History, Politics and Postmodernism. In: David Morley, Kuan-Hsing Chen (eds.): Stuart Hall. Critical Dialogues in Cultural Studies. London/New York: Routledge

Hall, Stuart (2000): Cultural Studies. Ein politisches Theorieprojekt. Ausgewählte Schriften 3, Hamburg

Hall, Stuart (1996): Introduction: Who Needs 'Identity'? In: Hall, Stuart/Paul du Gay (eds.): Questions of Cultural Identity. London u. a.

Haraway, Donna (1995): Situiertes Wissen. Die Wissenschaftsfrage im Feminismus und das Privileg einer partialen Perspektive. In: Dies.: Die Neuerfindung der Natur. Primaten, Cyborgs und Frauen. Frankfurt/New York

Haraway, Donna (1990): A Manifesto for Cyborgs: Science, Technology, and Socialist Feminism in the 1980s. In: Nicholson, Linda J.: Feminism/Postmodernism. New York/London

Harding, Sandra (1998): Is Science Multicultural? Postcolonialisms, Feminisms, and Epistemologies. Bloomington/Indianapolis

Harding, Sandra (1994): Das Geschlecht des Wissens. Frauen denken die Wissenschaft neu, Frankfurt/New York

Haywood, Chris/Mac an Ghaill, Máirtín (2003): Men and Masculinities - Theory, research and social practice. Buckingham-Philadelphia.

Kafka, Franz (1994): Tagebücher. Band 3: 1914-1923 (in der Fassung der Handschrift). Frankfurt a.M.

Kaltenecker, Siegfried (1998): Schwindelnde Männer. Neues zum Thema Männlichkeit. In: Widersprüche - Zeitschrift für sozialistische Politik im Bildungs-, Gesundheits- und Sozialbereich, Heft 67 (18. Jg.), Nr. 1

Knapp, Gudrun-Axeli (1992): Macht und Geschlecht. Neuere Entwicklungen in der feministischen Macht und Herrschaftsdiskussion. In: Gudrun-Axeli Knapp und Angelika Wetterer (Hg.): TraditionenBrüche. Entwicklungen feministischer Theorie, Freiburg

Laclau, Ernesto (1997): Inklusion, Exklusion und die Logik der Äquivalenz (Über das Funktionieren ideologischer Schließungen). In: Weibel, Peter/Žižek, Slavoj (Hg.): Inklusion : Exklusion. Probleme des Postkolonialismus und der globalen Migration, Wien

Meuser, Michael (1998): Geschlecht und Männlichkeit. Soziologische Theorie und kulturelle Deutungsmuster, Opladen

Meuser, Michael \& Behnke, Cornelia (1998): Tausendundeine Männlichkeit? Männlichkeitsmuster und sozialstrukturelle Einbindungen. In: Widersprüche - Zeitschrift für sozialistische Politik im Bildungs-, Gesundheits- und Sozialbereich, Heft 67 (18. Jg.), Nr. 1

Möller, Kurt (1997): Zur Einführung: Merkmale und Konturen sozialer und pädagogischer Arbeit am Männlichkeits-Spagat. In: Ders. (Hg.): Nur Macher und Macho? Geschlechtsreflektierende Jungen- und Männerarbeit, Weinheim/München 
Mouffe, Chantal (1997): Inklusion/Exklusion: Das Paradox der Demokratie. In: Weibel, Peter/Žižek, Slavoj (Hg.): Probleme des Postkolonialismus und der globalen Migration, Wien

Probyn, Elsbeth (1995): Queer Belongings. Eine Politik des Aufbruchs. In: Angerer, Marie-Luise (Hg.): The Body of Gender. Körper, Geschlechter, Identitäten, Wien

Straub, Jürgen: Personale und kollektive Identität. Zur Analyse eines theoretischen Begriffs. In: Assmann, Aleida \& Friese, Heidrun (Hg.): Identitäten - Erinnerung, Geschichte, Identität 3. Frankfurt a.M.

Stuve, Olaf (2001): „Queer Theory“ und Jungenarbeit. Versuch einer paradoxen Verbindung. In: Fritzsche, Bettina/Hartmann, Jutta/Schmidt, Andrea/Tervooren, Anja (Hg.): Dekonstruktive Pädagogik. Erziehungswissenschaftliche Debatten unter poststrukturalistischen Perspektiven, Opladen

Tillner, Georg/Kaltenecker, Siegfried (1995): Offensichtlich männlich. Zur aktuellen Kritik der heterosexuellen Männlichkeit. In: Texte zur Kunst, 5. Jg., Nr. 17

Walter, Willi (2000): Gender, Geschlecht und Männerforschung. In: Braun, Christina von \& Stephan, Inge (Hg.): Gender-Studien. Eine Einführung, Stuttgart \& Weimar

Whitehead, Stephen M. (2002): Men and Masculinities. Key Themes and New Directions. Cambridge: Polity

Wittels, Fritz: Die Feministen. In: Die Fackel, Nr. 248, 24. März 1908

Young, Iris Marion (1994): Geschlecht als serielle Kollektivität: Frauen als soziales Kollektiv. In: Geschlechterverhältnisse und Politik. Hg. vom Institut für Sozialforschung Frankfurt (Redaktion: Katharina Pühl). Frankfurt a.M.

Žižek, Slavoj (1994): The Sublime Object of Ideology. London/New York 


\title{
Vom Subjekt zur Kategorie. Veränderte Denkfiguren
}

\section{Eva Breitenbach}

\author{
„Das wahre Geheimnis der Welt ist das Sichtbare, \\ nicht das Unsichtbare“" \\ Oscar Wilde
}

In diesem Artikel setze ich mich mit Veränderungen und Ausdifferenzierungen der Kategorie ,Geschlecht' innerhalb der sozialwissenschaftlichen bzw. erziehungswissenschaftlichen Geschlechterforschung auseinander. Mein Ansatzpunkt sind Konfliktlinien, die mit einem Paradigmenwechsel - weg von subjektorientierten Konzeptionen hin zu (de)konstruktivistischen Positionen einhergehen.

Für die erziehungswissenschaftliche Geschlechterforschung gilt nicht nur, dass sie sich vor allem mit AkteurInnen in ihrem Alltag auseinandersetzt, sondern auch, dass sie stark handlungs- und praxisbezogen ausgerichtet war und ist. Darüber hinaus ist sie oft intentional mit einem Ziel verändernder oder emanzipatorischer Praxis verknüpft. Eine solche Ausrichtung lässt sich viel leichter an Paradigmen anschließen, die an Mustern von Ursache und Wirkung, Benachteiligung und Emanzipation, Gleichheit und Differenz, Wirklichkeit und Ideologie ausgerichtet sind als an solchen, die sich mit „schlichter Rekonstruktionsarbeit“ zu begnügen scheinen.

Die konstruktivistische Analyse ist demgegenüber ein empirisches Instrument, eine "Suchstrategie mit offenen Antwortmöglichkeiten“ (KnorrCetina 1997, S. 23), die zunächst keinerlei Aufforderung zu praktischem Handeln und nur wenig Anknüpfungspunkte dafür enthält. Eine konstruktivistische Geschlechterforschung hat die Sichtbarkeit des zweigeschlechtlichen Systems gegen sich, die Sichtbarkeit weiblicher und männlicher Personen und das individuelle und kollektive Bewusstsein geschlechtlicher Identität. Auch Überschreitungen der Zweigeschlechtlichkeit im gender crossing geschehen vor dem Hintergrund der zweigeschlechtlichen Ordnung. Menschen bedienen sich der geschlechtlichen Codierungen gerade auch dann, wenn sie sie verfremden oder überschreiten. „Aller Diversitäten und Unorthodoxien zum Trotz bleibt die Kategorie Geschlecht der einzige Referenzrahmen, und das nicht in erster Linie als Negativfolie.“ (Schröter 2002, S. 213). Darüber hinaus müssen Geschlechtsdarstellungen ,als ihre Konstruktivität verbergende Prozesse aufgefaßt werden“, um als erfolgreich und überzeugend gelten zu können (Hirschauer 1993, S. 41). Mit diesen „Naturalisierungsleistungen“ 
(Hirschauer 1993, S. 47f.) wird die Vereinbarkeit von Alltagswissen und Alltagshandeln mit der konstruktivistischen Analyse endgültig aufgegeben.

Ein Schwerpunkt der folgenden Ausführungen liegt auf den Veränderungen im Zusammenhang von Geschlecht und der Person bzw. ihrer Identität als einer wichtigen pädagogischen Fragestellung. Nach ,Identität' frage ich aus zwei Gründen: Einmal weil gerade die erziehungswissenschaftliche Geschlechterforschung nach wie vor auf den Begriff der "Geschlechtsidentität“ zurückgreift und genau dort ein Rückgriff auf Theorien mit einem essentialistischen Kern notwendig erscheint und zweitens weil ich die Frage nach der Geschlechtsidentität und der geschlechtlich identifizierbaren Person für einen bedeutsamen „Subtext“ in der Auseinandersetzung um verschiedene Spielarten von Geschlechterforschung halte: Ist Geschlecht etwas, das wir haben, tun oder sind? Und welche Konsequenzen hat die jeweilige Position? Einen zweiten Schwerpunkt bildet ein empirischer konstruktivistischer Ansatz. Ich schlage vor, das Geschlecht bzw. die Geschlechtszugehörigkeit als einen Erfahrungsraum bzw. als Teil eines Erfahrungsraums zu fassen, der die Grundlage einer spezifischen Orientierung und Praxis bildet. Geschlechtszugehörigkeit definiere ich grundlegend als eine Praxis, die empirisch (re)konstruierbar ist.

\section{Geschlecht als alltäglicher und wissenschaftlicher Besitzstand}

Im Paradigma der Geschlechterforschung wurde das Geschlecht zunächst als ein feststehendes Merkmal der Person begriffen, als eine körperliche und möglicherweise psychische Grundlage, die sozial und kulturell überformt wird. ${ }^{1}$ Im Mittelpunkt der Diskurse stand nicht die Kategorie Geschlecht, sondern die Frau in ihren aktuellen und historischen Lebenszusammenhängen. Dabei wurde die Frau als Benachteiligte und Unterdrückte konzipiert, als Person, der die Entfaltung ihres Potentials verwehrt wird (das führte zur Betonung von Defiziten), bzw. als eine Person, deren spezifische Potentiale ent-

1 Als Ausgangspunkt der feministischen Geschlechterforschung (die damals noch Frauenforschung hieß) können die 1970er Jahre mit der Neuen Frauenbewegung angesehen werden. Frauenforschung als feministisches Wissenschaftsprojekt gliedert sich in eine Vielzahl von theoretischen, methodischen und inhaltlichen Zugängen und Konzeptionen. Zusammengehalten wird sie durch ein lockeres Netz von grundlegenden Annahmen und Haltungen, nämlich Betroffenheit (als Frau), Parteilichkeit (mit anderen Frauen) und Kritik am Androzentrismus bzw. Patriarchatskritik. 
wertet werden (das führte zur Betonung von Differenzen) (vgl. Breitenbach/ Hagemann-White 1994). Auch wenn die Konzeption „der Frau“, die die Gemeinsamkeit der Frauen betont, von Anfang an kritisiert und ausdifferenziert wurde (in Ansätzen, welche die ethnischen, sozialen und kulturellen Unterschiede zwischen Frauen betonten oder in Ansätzen, die sich mit der Beteiligung der Frauen an ihrer Unterdrückung, mit ihrer Mittäterschaft auseinander setzten), so blieb doch die Geschlechtszugehörigkeit selbstverständlich an die Person gekoppelt, und zwar die weibliche Geschlechtszugehörigkeit an die Frau und die männliche Geschlechtszugehörigkeit an den Mann. Das Paradigma der Geschlechterverhältnisse als hierarchische oder als Herrschaftsverhältnisse stützte sich grundlegend auf die Konzeption der Zweigeschlechtlichkeit. Eine zentrale Frage innerhalb dieses Paradigmas war die Frage nach Ursachen (von Herrschaft und Unterdrückung) und Wirkungen (auf die Unterdrückten wie die Unterdrücker). Solche Analysen ließen sich oft mehr oder weniger bruchlos mit einer Aufforderung zu emanzipatorischer Praxis verbinden.

Am Thema „Gewalt im Geschlechterverhältnis“ lassen sich theoretische Konflikte zwischen einer konstruktivistischen Geschlechterforschung auf der einen Seite und einer handlungsorientierten feministischen Geschlechterforschung auf der anderen Seite illustrierend aufzeigen. ${ }^{2}$ Die feministische Geschlechterforschung konzipierte das Geschlechterverhältnis als Herrschaftsverhältnis und sah in individueller Gewalt von Männern gegen Frauen ein entscheidendes Instrument zu seiner Erhaltung. Diese theoretische Konzeption implizierte die normative Setzung von Recht und Unrecht und die Forderung nach individueller wie nach gesellschaftlicher Gerechtigkeit. Die Veröffentlichung der „unterdrückten Wahrheit“ galt sowohl als wissenschaftliche wie auch als politische Notwendigkeit. Für eine solche Art von Forschung sind Personen als Akteure und als Adressaten zentral. Unterdrückt werden nicht Kategorien, sondern Menschen und zwar geschlechtlich identifizierbare Menschen.

Diejenigen feministischen ForscherInnen, für die die Aufdeckung von Gewaltverhältnissen ein notwendiger Bestandteil der Suche nach Gerechtig-

2 (Sexuelle) Gewalt im Geschlechterverhältnis, die Diskriminierung, Unterdrückung und körperliche wie psychische Verletzung aufgrund des Geschlechts war immer ein zentrales Thema der feministischen Geschlechterforschung bzw. Frauenforschung, ist aber bislang nicht zu einem wichtigen Thema universitärer, akademischer Geschlechterforschung geworden. Das könnte sich allmählich ändern. Immerhin erscheinen derzeit eine repräsentative Studie über Gewalt gegen Frauen in Deutschland (Projektleitung Ursula Müller und Monika Schröttle) und der Endbericht der wissenschaftlichen Begleitung von Interventionsprojekten gegen häusliche Gewalt (Projektleitung Carol Hagemann-White und Barbara Kavemann). 
keit zwischen den Geschlechtern und Generationen darstellt, sehen die Essenz ihrer Arbeit durch die Relativierungen einer interaktionistischen oder konstruktivistischen Analyse bedroht.

„Interaktionistische Argumente können einem Gewaltforscher wie dem Verfasser ganz schön auf den Geist gehen. Wenn Verstand und Herz nach jahrelangem Studium der Ursachen, Ausprägungen und Folgen von Gewalt einigermaßen zerbrechlich geworden sind und dann behauptet wird, Gewalt sei ein Mythos, sei nicht messbar, sei ein interaktionelles Geschehen, es gäbe weder Täter noch Opfer (vor allem in der Therapie eine für die Misshandelten folgenschwere Position), dann können Unlustgefühle aufsteigen." (Godenzi 1994, S. 94) Umgekehrt stellen die umstandslosen Setzungen von Männern, Frauen, Tätern und Opfern und vor allem von weiblichen und männlichen Körpern für eine konstruktivistische akademische Forschung völlig unakzeptable Kategorien dar.

Versuche, eine konstruktivistische Theorie von Gewalt im Geschlechterverhältnis auszuarbeiten, gibt es bislang leider so gut wie nicht. Das könnte auch daran liegen, dass hier Menschen als Menschen und als Opfer oder Täter thematisiert werden. Das Thema bringt ForscherInnen in Kontakt mit den bedrohlichen und dunklen Seiten ihrer leiblichen weiblichen oder männlichen Existenz, es identifiziert sie mit ihrem Geschlecht. In der Ablehnung dieser Identifikation von Geschlecht und Person auf der einen Seite und der Notwendigkeit dieser Identifikation für die theoretische wie die praktische Arbeit andererseits, liegt ein zentraler Unterschied zwischen den verschiedenen Forschungszugängen und ein Kern des Konflikts. Die Aussage von Godenzi macht sehr deutlich, dass es bei derartigen verschiedenen Positionen auch um Gefühle geht, um eine emotional besetzte Sicht der Welt. Es geht um den „zerbrechlichen Verstand“ und das „,zerbrechliche Herz“, die von Sichtweisen, die die eigenen Grundlagen ablehnen bzw. zerstören, irritiert und verletzt werden können.

\section{Von der Frau zur Kategorie Geschlecht: Veränderte Denkfiguren}

Einflussreiche Spielarten der Geschlechterforschung haben Veränderungen bewirkt, von denen ich zwei entscheidende nennen möchte: Zum einen haben sie die selbstverständliche Verbindung von Geschlechtszugehörigkeit und Person aufgebrochen. Damit sind nicht nur Fragen des Geschlechts angespro- 
chen, sondern auch Fragen von Identität. Zum zweiten verschiebt die konstruktivistische Geschlechterforschung die Fragestellung vom warum zum wie: Sie fragt danach, wie Geschlechter und ihre Verhältnisse hergestellt werden. „Nicht die sozialen Konsequenzen der angeborenen Geschlechtsunterschiede bedürfen also einer Erklärung, sondern vielmehr wie diese Unterschiede als Garanten für unsere sozialen Arrangements geltend gemacht wurden (und werden) und, mehr noch, wie die institutionellen Mechanismen der Gesellschaft sicherstellen könnten, dass uns diese Erklärungen stichhaltig erscheinen" (Goffman 1994, S. 107).

Seit den neunziger Jahren werden empirische Forschung und Theoriebildung in der Geschlechterforschung von Paradigmen der Konstruktion oder Dekonstruktion von Geschlecht dominiert. Bei aller Verschiedenheit der einzelnen Konzeptionen wird Geschlecht hauptsächlich oder ausschließlich als soziale und kulturelle Konstruktion, als soziale und kulturelle Praxis aufgefasst. Auch Körpererfahrungen und Körperwissen werden als kulturelle Praktiken diskutiert. Bestenfalls gilt der Körper als „Teilnehmer im sozialen Geschehen“ (Connell 1999, S. 80), dessen Authentizität allerdings in Frage steht.

Damit hat die Geschlechterforschung die Reflexion ihrer eigenen Denkgewohnheiten, Kategorien und Konzepte in einer außerordentlich radikalen Weise betrieben. Sie hat ihre zentrale Kategorie ,Geschlecht' in der griffigen Formel des doing gender verflüssigt. Sie hat die Verbindungen zwischen der Person und der Geschlechtszugehörigkeit gelockert oder aufgelöst. Sie hat in ihren Kontextanalysen den Stellenwert der Kategorie Geschlecht gegenüber anderen Kategorien wie der ethnischen Zugehörigkeit und dem sozialen Milieu relativiert. Diese theoretische und empirische Arbeit, die bis zum Anfang der eigenen Grundlagen und damit bis zum Ende der Gewissheiten führt, trägt sicherlich zur Lebendigkeit und Kreativität der Geschlechterforschung bei.

Sie führt aber gleichzeitig zu neuen Schwierigkeiten oder Herausforderungen. Eine Herausforderung, die ich im folgenden diskutieren werde, liegt in der Verbindung der Konzeption des doing gender mit anderen sozialwissenschaftlichen oder auch dezidiert erziehungswissenschaftlichen Theoriebeständen. 


\section{Gender als soziale Institution. Der Ansatz von Judith Lorber}

Der Ansatz von Judith Lorber verbindet eine sozialkonstruktivistische Auffassung von gender mit einer Analyse der Geschlechterverhältnisse als Herrschaftsverhältnisse und einem dezidierten Ziel: „Wenn die gender-Ungleichheit abgeschafft werden soll, müssen die gender entweder in jeder Hinsicht völlig gleich gestellt werden, oder gender darf nicht länger eine zentrale Kategorie sein, die bestimmt, welcher soziale Status einem Menschen zugewiesen wird" (Lorber 2003, S. 7). Das Projekt, das sie vorschlägt, besteht in degendering oder undoing gender als dem ,ultimativen feministischen Ziel“ (Lorber 2003, S. 7). ${ }^{3}$

Lorber betrachtet gender ausschließlich als soziale Konstruktion. Soziale Konstruktion bedeutet, dass gender individuell wie interaktiv wahrgenommen und dargestellt wird und werden muss: Diese soziale Praxis ist unausweichlich. Lorber setzt jedoch nicht bei den Individuen oder den sozialen Beziehungen an, auch wenn sich „Konstruktion und Aufrechterhaltung von gender in den persönlichen Identitäten und in der sozialen Interaktion manifestieren“ (Lorber 2003, S. 41). Vielmehr konzipiert sie gender als zentrale soziale Institution und sieht darin ein neues Paradigma. Gender als soziale Institution durchdringt und strukturiert alle Bereiche sozialer Praxis wie Wirtschaft, Politik, Familie und Sexualität und ist selbst eine mächtige und grundlegende Struktur. Als sozialer Platzanweiser ist gender ein Instrument zur Herstellung und Sicherung von hierarchischer Ungleichheit und Herrschaft zwischen den Geschlechtern. Als Institution ist gender dem individuellen Handeln zwar vorgeordnet, aber nicht im Sinne einer inhaltlichen Festlegung. Gender ist sozusagen eine dynamische Struktur und gerade in ihrer Flexibilität so zählebig. Diese Flexibilität einerseits und das Beharrungsvermögen vergeschlechtlicher sozialer Praxis andererseits vermag Lorber materialreich zu belegen, z.B. in ihrer sehr anregenden und fruchtbaren Analyse sexueller Verhältnisse. ${ }^{4}$

3 Ähnlich argumentiert Robert Connell in seiner Konzeption zur Konstruktion von Männlichkeit, wobei sich die beiden AutorInnen nicht systematisch aufeinander beziehen.

4 Lorber betrachtet Sexualität konsequent als einen Lernprozess kulturell vermittelter ,Skripte“, auch wenn sie von natürlichen Unterschieden zwischen Frauen und Männern ausgeht. Sie zeigt, in welchem Ausmaß kulturelle Skripte sexuelle Praktiken (und Phantasien) definieren, normieren, beschränken bzw. als ,abweichend“ definieren, allen voran die zwangsweise Verbindung von Sexualität mit Genitalität. Mit ihrer konsequenten Trennung von sex, gender und Sexualität zeigt sie die Notwendigkeit für die Individuen auf, sich innerhalb eines restriktiven und beschränkten Repertoires selbst zu klassifizieren und zu verorten. 
Nicht theoretisiert wird, wie Produktions- und Vermittlungsprozesse im einzelnen verlaufen, wie z.B. „Kulturen und Gesellschaften die Sexualpraktiken in gebilligte, erlaubte und tabuisierte Muster einteilen, die von den Individuen verinnerlicht werden“ (Lorber 2003, S. 108). Diese Fragen bleiben den einzelnen empirischen Rekonstruktionen überlassen. Daran könnte es liegen, dass in den empirischen Beschreibungen die zweigeschlechtliche Ordnung als eine außerordentlich festgefügte Struktur erscheint, innerhalb derer die Geschlechtszugehörigkeit quasi unter der Hand naturalisiert wird und den Status der zentralen Kategorie erhält. Dies ist sicherlich ein wichtiges Problem derjenigen sozialkonstruktivistischen Ansätze, die gender als Institution und Struktur definieren. So weist Sedef Gümen darauf hin, dass sich auch in den Theoriesträngen, die die interaktive Herstellung des Geschlechts thematisieren, quasi unter der Hand die Geschlechterdifferenz als zentrale Differenz durchsetze.

Andere Formen der Differenz werden damit zum Besonderen vor dem diesem Allgemeinen. Die Ansprüche nach Pluralisierung von Differenzen und nach Kontextualisierung der Kategorie Geschlecht als einer grundlegend im Sozialen eingebundenen Kategorie würden damit unterlaufen. „Aus dieser Perspektive gelten andere soziale Kategorien, wie Klassenzugehörigkeit, ethnisierende und rassistische Markierungen als sekundäre oder konkurrierende Merkmale“ (Gümen 1996, S. 79, vgl. auch Gümen 1998).

\section{Doing gender. Geschlecht als Praxis}

In den mittlerweile bereits klassisch zu nennenden Ansätzen, die in der Konzeption des doing gender gebündelt werden, wird Geschlecht als eine grundlegend soziale Kategorie angesehen. Geschlecht wird zunächst als situativ und interaktiv hergestellt betrachtet, es wird situativ und interaktiv thematisch oder auch nicht. „Konstruktion von Geschlecht“ meint die Darstellungsprozesse und Wahrnehmungsprozesse von Geschlecht (vgl. z.B. Goffman 1994, Hirschauer 1993). Doing gender sind alle Tätigkeiten, durch die Geschlecht dargestellt wird, alle Tätigkeiten, durch die Geschlecht wahrgenommen wird. Das schließt diejenigen Tätigkeiten ein, mit deren Hilfe die Geschlechterdifferenzen dargestellt und wahrgenommen werden. Diesen Tätigkeiten liegen kulturelle Wissensbestände über das Geschlecht zugrunde beispielsweise über die Unausweichlichkeit und Ausschließlichkeit der Geschlechtszugehörigkeit, aber auch über Objekte und Praktiken, die zur Konstruktion von Geschlecht 
genutzt werden können oder müssen. Goffman (1994) hat deutlich gemacht, dass es sich dabei um zirkuläre Prozesse institutioneller Reflexivität handelt, die - vom Einzelnen aus gesehen - bei der Geburt mit der Zuordnung zu einer Geschlechtsklasse ihren Anfang nehmen und dazu dienen, die Gesellschaftsmitglieder in der Institution „Geschlecht“ zu verorten.

Zentral wird hier, wie schon ausgeführt, die Frage nach dem wie, die die Frage nach dem warum ablöst. Wie wird Geschlecht konstruiert? Wie sehen die Praktiken aus, mit denen Menschen sich als weiblich oder männlich wahrnehmen und darstellen? Auf welche Bilder, Objekte, Stile, Tätigkeiten und Erzählungen können Individuen und Gruppen zurückgreifen? Wie sehen die Praktiken aus, mit deren Hilfe die Konstruktionsprozesse ihrerseits unkenntlich gemacht werden, so dass uns das System der Zweigeschlechtlichkeit als (Natur)tatsache entgegentritt und das Geschlecht eines Menschen ihm selbst und anderen als kultureller oder natürlicher Besitz erscheint?

Welche Praktiken als doing gender gefasst werden können, ist eine Frage, auf die die empirische Analyse eine Antwort geben kann. In unserer Forschung über jugendliche Beziehungskulturen fielen darunter das geschlechtlich codierte Dekor wie Kleidung und Haartracht, diverse geschlechtlich codierte kindliche, jugendliche und erwachsene Aktivitäten, aber auch so komplexe Tätigkeiten wie die Entwicklung und Aufrechterhaltung einer angemessenen sexuellen Praxis und nicht zuletzt die Erzählungen über Geschlecht. ${ }^{5}$ Die konkreten empirischen Beschreibungen in der Kindheits- und Jugendforschung zeigen, dass es sich um hochkomplizierte emotional besetzte individuelle wie kollektive Prozesse handelt, die dazu beitragen, der Person ein Bewusstsein von sich selbst und dem eigenen Ort zu verleihen, eben (geschlechtliche) Orientierung und Identität. Aber dieser empirisch nachvollziehbare Schritt lässt sich in der Konzeption des doing gender nicht mehr explizit vollziehen. Das konstruktivistische Analyseinstrumentarium löst die Geschlechtszugehörigkeit in ihre einzelnen Tätigkeiten auf, setzt die Bestandteile aber nicht mehr zu einer Identität zusammen.

Gerade dadurch könnte aber wieder Dynamik in einen Prozess kommen, der ansonsten in der Gefahr ist, Konstruktionsprozesse in einem Repertoire festgelegter Handlungen erstarren zu lassen.

5 Ich beziehe mich auf ein mehrjähriges Forschungsprojekt über freundschaftliche Beziehungen unter Jugendlichen, untersucht in Mädchen- und Jungengruppen (vgl. Breitenbach 2000, Breitenbach/Kausträter 1999, 2004, Kausträter 2004). Ich möchte mich an dieser Stelle bei Sabine Kausträter für ihre Unterstützung bei der Arbeit an diesem Aufsatz bedanken. Ebenfalls bedanke ich mich bei Petra Korte und bei Leonie Herwartz-Emden. 


\section{Doing Adolescence. Jugend als Praxis}

Erziehungswissenschaft und erziehungswissenschaftliche Geschlechterforschung hantieren mit Theoriebeständen, die eine biologische oder kulturelle essentielle Differenz enthalten, allen voran die Differenz zwischen Kindheit, Jugend und Erwachsenheit und die ganz grundlegend die Person in ihren Mittelpunkt stellen, die interagierende, $\mathrm{zu}$ erziehende, $\mathrm{zu}$ vergesellschaftende, bildsame Person, die eine (geschlechtliche) Identität zu entwickeln hat.

Wie können diese subjekt- und lebensphasenbezogenen Theorien mit einer konstruktivistischen Auffassung von Geschlecht verbunden werden? Wo ist der Übergang vom Tun des Geschlechts zum Haben des Geschlechts bzw. der Geschlechtsidentität? Das ist auch deshalb eine notwendige Frage, weil „Konstruktionen von Geschlecht“ nie isoliert auftreten sondern stets eingebunden in soziale Prozesse und Beziehungen: So sind die Konstruktionsprozesse von Geschlecht bei Jugendlichen immer gebunden an die Altersangemessenheit der Darstellung, an den entsprechenden Kontext und an das soziale Milieu. Und es ist eine glücklicherweise vorläufig noch offene Frage. Glücklicherweise deshalb, weil ich glaube, dass diese Frage keine endgültige theoretische Lösung und damit Schließung verträgt. Derzeit sehe ich drei Möglichkeiten: erstens die konkrete empirische Rekonstruktion und Modifikation theoretischer Konzepte, zweitens die genaue Untersuchung von Anschlussmöglichkeiten und -grenzen und drittens, und das möchte ich im Folgenden an der Theorie von ,Jugend' erläutern, eine Veränderung oder Verflüssigung der theoretischen Paradigmen in einem konstruktivistischen Prozess.

Die Denkfigur der Konstruktion scheinbar essentieller und stabiler Phänomene lässt sich mit der Konzeption von Jugend verknüpfen. Die Aussagen über die Konstruktionsprozesse des Geschlechts lassen sich mit einer gewissen Plausibilität gewissermaßen ,probehalber' auf die Konstruktionsprozesse von Jugendlichkeit übertragen bzw. damit verbinden. Jugend muss ebenso wie Geschlecht und in Zusammenhang damit überzeugend dargestellt und wahrgenommen werden.

Jugend ist weder als Personengruppe, noch als Lebensphase oder als gesellschaftliche Institution eindeutig und einheitlich zu erfassen. Die Vorstellung einer Jugendphase als eigenständigem und abgrenzbarem Lebensabschnitt erodiert von den ,Rändern' her, dem zunehmend weniger festgefügten Anfang und vor allem Ende der Jugendphase. Jugendliche Mitglieder schillernder Subkulturen, Jugendliche verschiedener ethnischer Herkunft und $\mathrm{Zu}$ gehörigkeit und unterschiedlichster sozialer und ökonomischer Lage sind zu- 
dem zunehmend schwerer als eine Gruppe mit tragfähigen Gemeinsamkeiten zu beschreiben. Auf der anderen Seite stehen Jugendlichen heute erhebliche zeitliche, materielle und kulturelle Ressourcen zur Verfügung, um Jugend in Abgrenzung von Kindheit und Erwachsenenalter darzustellen. Wie sich Jugend für Jugendliche letztendlich ausgestaltet, dokumentiert sich in den Beschreibungen von Jugendlichen und wird erst in der Analyse begrifflich fassbar.

In unserer Jugendforschung zeigten sich zwei Dimensionen des Umgangs mit der Geschlechtszugehörigkeit. Die Geschlechtszugehörigkeit und die Geschlechterdifferenz (bzw. die Geschlechterunterscheidung) erwiesen sich als eine bedeutsame Ressource bei der Bewältigung des Alltags, z.B. des schulischen Alltags oder bei der Bewältigung jugendlicher Probleme. Kinder und Jugendliche greifen darauf mit einem gewissen spielerischen Vergnügen, aber auch mit tiefer Selbstverständlichkeit über ihre Geltung zurück. Gleichzeitig zeigt sich die Notwendigkeit und der Zwang, das Geschlecht in überzeugender Weise darzustellen. Gerade für Jugendliche ist das ein höchst komplizierter Vorgang und schließt die Notwendigkeit ein, auf sexuierte Räume, Objekte und Praktiken zurückzugreifen. Jugendliche nutzen ebenfalls biographisch gewachsene Orientierungen und erworbene Kompetenzen durchaus auch geschlechtstypischer Art zur Darstellung des Geschlechts:

So tanzen Mädchen, weil sie Mädchen sind und auch, um Mädchen zu sein. Und Jungen spielen Fußball, weil sie Jungen sind und um Jungen zu sein. Weibliche und männliche Jugendliche praktizieren Heterosexualität auch deshalb, weil dies zu einer angemessenen Darstellung von Jugendlichkeit gehört.

In einer solchen Auffassung von Jugend und Geschlecht ist die Geschlechtsidentität nichts, das in Auseinandersetzung mit den körperlichen Vorgängen der Pubertät und den gesellschaftlichen Anforderungen ,angeeignet" werden muss und auf geheimnisvolle Weise aus den Tiefenschichten der Person destruiert bzw. stabilisiert wird. Wenn Geschlecht in Bildern und Tätigkeiten dargestellt und wahrgenommen wird, dann könnte die Geschlechtsidentität mithilfe von Erzählungen dieser Tätigkeiten und Beschreibungen der Bilder zusammengehalten werden. „Die narrative Identität ist dementsprechend eine überaus dynamische Struktur. Die Erzählung konstituiert Identität als Einheit der Person, ohne zu unterschlagen, daß diese Einheit eine stets fragile und beständig zu reproduzierende Synthese des Differenten und Heterogenen ist" (Straub 2000a, S. 173). ${ }^{6}$ Die Geschlechtsidentität wäre dann die reflexive Selbstbeschreibung und Selbstthematisierung der Person, die in ih-

6 Jürgen Straub selbst bezieht sich in seinen Ausführungen nicht auf Fragen des Geschlechts. 
ren (individuellen und kollektiven) Erzählungen, durch „narrative Sinnbildungsleistungen“" Kontinuität und Kohärenz gewinnt (vgl. Straub 2000b, S. 283). An diesem Ort der Identitätsarbeit könnte der Übergang liegen von, Geschlecht haben' und ,Geschlecht tun'. Die Frage ,wer bin ich und wer möchte ich sein" als Kernfrage der Identität (vgl. Straub 2000b, S. 279) schließt dann Geschichten über die geschlechtliche Praxis mit ein.

\section{Geschlecht als Erfahrungsraum}

Ein grundlegendes Problem empirischer Forschung ist darin zu sehen, dass die empirische Analyse, die Geschlechterdifferenzen untersucht, von vornherein von Geschlechterdifferenzen ausgeht, also von Männern und Frauen, Mädchen und Jungen, und auf dieser Basis selbstverständlicher Zweigeschlechtlichkeit Ähnlichkeiten und Unterschiede sucht. ,Geschlechtszugehörigkeit' ist dann eng verbunden mit Geschlechterdifferenz und meint die alltägliche Zuordnung zum männlichen oder weiblichen Geschlecht innerhalb der zweigeschlechtlichen Ordnung. Hier trifft sich die Konzeption der Geschlechtszugehörigkeit als sozialer Konstruktion mit der Konzeption der Geschlechtszugehörigkeit als Basis der Persönlichkeit. Aus dem hier enthaltenen Zirkel kann empirische Forschung nicht entkommen. Der Gefahr der Reifizierung von Differenz kann die Forscherin in zweierlei Hinsicht begegnen. Einmal durch eine sorgfältige methodische Kontrolle der Dimension Geschlecht wie sie eine rekonstruktive Sozialforschung vorsieht. Zum Zweiten dadurch, dass Geschlechtstypik im Sinne eines sozialen Geschlechts nicht an das biologische Geschlecht gebunden ist.

Die empirische Rekonstruktion kann für bestimmte Fragestellungen zeigen, ob hier die Geschlechtszugehörigkeit eine relevante existentielle Kategorie darstellt, ob sie also Teil eines Erfahrungsraumes ist, wie dieser Erfahrungsraum ausgestaltet ist und schließlich, welche Orientierungen diese spezifische Ausgestaltung hervorbringt. ${ }^{7}$ Wir haben Mädchengruppen als weiblichen Raum beschrieben (einen konjunktiven Erfahrungsraum besonderer Art), der sich über eine spezifische Praxis konstituiert. Dieser weibliche Raum kann im Einzelfall (tatsächlich wurde uns ein solcher Fall geschildert) durchaus ein männliches Mitglied beherbergen. Umgekehrt stellt nicht jede Mädchengruppe einen weiblichen Erfahrungsraum her. Die Geschlechtszuge-

7 Mit dem Konzept des „Erfahrungsraums“ beziehe ich mich auf die Arbeiten von Ralf Bohnsack. 
hörigkeit ist also nur eine Dimension der Möglichkeiten, einen weiblichen Raum zu schaffen (vgl. Breitenbach 2000).

Die Geschlechtszugehörigkeit als Erfahrungsraum kann die Gestalt eines Handlungsraums und Spielraums annehmen, aber auch eines Käfigs oder Gefängnisses. Die Analyse kann zeigen, ob und in welcher Weise die Geschlechtszugehörigkeit und die Geschlechterdifferenz bestimmte Erfahrungen und Orientierungen beeinflussen oder konstituieren. Die Bedeutungen der Geschlechtszugehörigkeit können für bestimmte Erfahrungen und Orientierungen beschrieben werden, auch für solche Erfahrungen und Orientierungen, die Teil der Geschlechtsidentität werden oder sind. Das heißt aber nicht, dass die Geschlechtszugehörigkeit in ihren Bedeutungen selbst festgelegt und definiert werden kann oder an einem bestimmten Ort festgesetzt werden kann. Eine solche Festlegung würde eine Reifizierung der Geschlechterdifferenz bedeuten.

\section{Empirischer Konstruktivismus und konstruktivistische Empirie}

„Objektivität ist die Wahnvorstellung, Beobachtungen könnten ohne Beobachter gemacht werden. Die Berufung auf Objektivität ist die Verweigerung von Verantwortung - daher auch ihre Beliebtheit“ (Förster, zitiert nach Maturana/Pörksen 2002, S. 35). In der Denktradition des Konstruktivismus wird der wissenschaftliche Erkenntnisprozess als in der sozialen Praxis verankert angesehen. Er ist selbst eine soziale Praxis. Soziale Einflüsse im Forschungsprozess sind deshalb nicht als Störfaktoren aufzufassen. Vielmehr ist es notwendig, die Wirkungen sozialer Einflüsse auf das wissenschaftliche Denken selbst zu untersuchen (vgl. Knorr-Cetina 1997), um solche Einflüsse auf reflektierte Weise als Erkenntnisinstrumente nutzen zu können. Dies ist eine Folge der konsequenten Betrachtung von Wissenschaft als sozialer Praxis, die sich nicht auf eine ,objektive“ Instanz berufen kann. Eine zweite Folge ist, dass Methoden nicht aus Methodologien und Methodologien nicht aus logischen Systemen abgeleitet werden können. Die Begründung von Methoden erfolgt vielmehr auf dem Weg der Rekonstruktion von wissenschaftlicher und außerwissenschaftlicher Praxis. Diesen Weg bezeichnet Bohnsack als praxeologische Fundierung von Methoden (vgl. Bohnsack 1999, S. 92). „Dabei geht es im wesentlichen um die umfassende Verankerung von Methoden in der sozialen Praxis und um ihre methodologische Begründung auf dem Wege der 
Rekonstruktion dieser Praxis - sowohl der als wissenschaftlich wie der als außerwissenschaftlich verstandenen Praxis“ (Bohnsack 1999, S. 192, Hervorh. im Orig.).

Das Suchinstrument eines empirischen Konstruktivismus ist darauf gerichtet, „wie soziale Gruppierungen sich selbst choreographieren und ihre Choreographien tanzen“ (Knorr-Cetina 1989, S. 93). Die Verschiebung der Fragestellung auf den Herstellungsprozess oder die Choreographie des Sozialen verleiht dem Forschungsprozess Offenheit und Dynamik. Das findet eine interessante Entsprechung im Bild des ,Tanzes' sozialer Gruppierungen. Entscheidend scheint zu sein, dass die Dynamik des Forschungsprozesses auch seine Ergebnisse kennzeichnet. Die Untersuchung des ,Herstellungsprozesses ${ }^{6}$ kann dazu verleiten, als Ergebnis ein in Definitionen festgefrorenes Endprodukt festzuhalten. Diese Gefahr ist umso stärker, je linearer sich der Denkund Forschungsprozess gestaltet und ganz im Sinne eines linearen Denkens in einem Ziel endet. Wenn die Forschung zusätzlich den Anspruch hat, die Erfahrungsräume oder existentiellen Hintergründe der Forschungsadressaten zu erschließen, also die Choreographien quasi existentiell zu verankern, ergibt sich die Gefahr einer zumindest impliziten Objektivierung von Forschungsergebnissen, die Differenzierung von interpretierten und ,gegebenen' Realitäten. Diese Problematik lässt sich nur in der jeweils konkreten empirischen Arbeit bearbeiten.

Weiterführend für die Geschlechterforschung ist meines Erachtens die konkrete empirische (Re)Konstruktion des Geschlechts als Praxis einschließlich ihrer Rekonstruktion als basale Dimension im Forschungsprozess selbst.

\section{Literatur}

Bohnsack, Ralf (1999): Rekonstruktive Sozialforschung. Einführung in Methodologie und Praxis qualitativer Forschung, Opladen

Breitenbach, Eva (2000): Mädchenfreundschaften in der Adoleszenz. Eine fallrekonstruktive Untersuchung von Gleichaltrigengruppen, Opladen

Breitenbach, Eva/Hagemann-White, Carol (1994): Von der Sozialisation zur Erziehung - Der Umgang mit geschlechtsdifferenter Subjektivität in der feministischen Forschung, in: Beutler, Kurt et al. (Hg.): Die Geschlechterverhältnisse und die Pädagogik. Jahrbuch für Pädagogik 1994, Frankfurt am Main

Breitenbach, Eva/Kausträter, Sabine (1999): „Einarbeiten in heterosexuelle Umgangsformen“: Zur Bedeutung von Mädchenfreundschaften in der Adoleszenz, in: Horstkemper, Marianne/Kraul, Margret (Hg.): Koedukation. Erbe und Chancen, Weinheim 
Breitenbach, Eva/Kausträter, Sabine (2005): Wege durch die Adoleszenz. Eine Typologie jugendlicher Beziehungskulturen: Kommunikation, Aktionismus und Zielorientierung, erscheint in: Bohnsack, Ralf / Przyborski, Aglaja / Schäffer, Burkhard (Hg.): Das Gruppendiskussionsverfahren in der sozialwissenschaftlichen Praxis, Opladen

Connell, Robert William (1999): Der gemachte Mann. Konstruktion und Krise von Männlichkeiten, Opladen

Goffman, Erving (1994): Das Arrangement der Geschlechter, in: Ders.: Interaktion und Geschlecht, Frankfurt am Main u.a.

Godenzi, Alberto (1994): Gewalt im sozialen Nahraum, Basel u.a.

Gümen, Sedef (1996): Die sozialpolitische Konstruktion „kultureller“ Differenzen in der bundesdeutschen Frauen- und Migrationsforschung, in: Beiträge zur feministischen Theorie und Praxis, Heft 42

Gümen, Sedef (1998): Das Soziale des Geschlechts. Frauenforschung und die Kategorie „Ethnizität“, in: Das Argument, Heft 1,2

Hirschauer, Stefan (1993): Die soziale Konstruktion der Transsexualität. Über die Medizin und den Geschlechtswechsel, Frankfurt am Main

Kausträter, Sabine (2005, in Vorbereitung): Jungenfreundschaften. Zur Konstruktion von Männlichkeit in der Adoleszenz

Knorr-Cetina, Karin (1989): Spielarten des Konstruktivismus. Einige Notizen und Anmerkungen. In: Soziale Welt (Sonderband)

Knorr-Cetina, Karin (1997): Konstruktivismus als „Strategie der Weltentfaltung“, in: Bardmann, Theodor M. (Hg.), Zirkuläre Positionen: Konstruktivismus als praktische Theorie, Opladen

Lorber, Judith (2003): Gender-Paradoxien, Opladen

Maturana, Humberto R./Pörksen, Bernhard (2002): Vom Sein zum Tun. Die Ursprünge der Biologie des Erkennens, Heidelberg

Nicholson, Linda (1994): Was heißt ,gender“? In: Institut für Sozialforschung Frankfurt (Hg.): Geschlechterverhältnisse und Politik, Frankfurt am Main

Schröter, Susanne (2002): FeMale. Über Grenzverläufe zwischen den Geschlechtern, Frankfurt am Main

Straub, Jürgen (2000a): Identitätstheorie, empirische Identitätsforschung und die „postmoderne“ armchair psychology, in: ZBBS, Zeitschrift für qualitative Bildungs-, Beratungs- und Sozialisationsforschung, Heft 1

Straub, Jürgen (2000b): Identität als psychologisches Deutungskonzept, in: Greve, Werner (Hg): Psychologie des Selbst, Weinheim 


\section{Zur Lage der pädagogischen Frauen- und Geschlechterforschung: bildungstheoretische Diskussionen}

\section{Fritjof Bönold}

Die Lage erscheint als durchaus erfreulich. Die Fragestellungen und theoretischen Zugänge der erst jungen pädagogischen Frauen- und Geschlechterforschung erweitern sich ständig; ihr Gegenstand gewinnt zunehmend an Gestalt. Mit einer Reihe von in der Zwischenzeit klassischen Themen und ersten Lehrbüchern findet auch schon eine erste Traditionsbildung statt. ${ }^{1}$ Der inhaltlichen Ausgestaltung, den Zugewinnen an Perspektiven und der Verfestigung eines ersten Kerncurriculums steht zudem ein Prozess der Verwissenschaftlichung bzw. Professionalisierung zur Seite. So wird auch schon von einer „,neuen Generation“ von Forschenden gesprochen, die sich gegenüber „den Älteren" beginnen zu profilieren.

Betrachtet man die Situation nun näher, werden spezifische Spannungen sichtbar. So zunächst innerhalb der Disziplin. Hier ist die Lage der pädagogischen Frauen- und Geschlechterforschung weiterhin prekär. Der neutrale bzw. androzentrische Hauptstrom der Erziehungswissenschaft nimmt bis heute nur episodenhaft die Forschungsergebnisse wahr und der Austausch bleibt unerfreulich beschränkt. ${ }^{2}$ Dies steht im deutlichen Kontrast zu der Tatsache, dass, neben hochschulpolitischen Themen und Fragen der Schulreform (heute: PISA), die Koedukationsproblematik in den letzten 15 Jahren ein ganz zentrales pädagogisches Thema war, das von der Öffentlichkeit aufgegriffen wurde.

Die Lage der Forschung wäre aber unzureichend gekennzeichnet, würde nicht auch ihr spezifisches Spannungsverhältnis zur pädagogischen Praxis angesprochen. Hier befindet sich die pädagogische Frauen- und Geschlechterforschung zunächst auf der für die Anwendungsorientierung der Disziplin typischen Gratwanderung zwischen parteilicher Hilfe und neutraler Beobach-

1 Siehe Faulstich-Wieland (1995, 2003), Nyssen/Schön (1995), Wolf (1996), Kleinau/Opitz (1996), Friebertshäuser/Jacob/Klees-Möller (1997), Friebertshäuser/Prengel (1997), Krüger (1997), Rendtorff/Moser (1999), Lemmermöhle u.a. (2000), Gieseke (2001) oder Fleßner/ Kaiser (2002).

2 Siehe Faulstich-Wieland (1995) und Priem (1999). Zu positiven wie negativen Episoden siehe Schäfer (1988), Preuss-Lausitz (1991), Krüger (1997), Drerup (1997), FaulstichWieland (1998) und Tenorth (1999). 
tung der Praxis. Dieser Balanceakt ist dabei aber besonders gefährdet durch ,männliche' Vorwürfe der Unwissenschaftlichkeit aus der Disziplin, aber auch durch vorschnelle Forderungen aus der Praxis. Durch ihre Geschichte befindet sich die pädagogische Frauen- und Geschlechterforschung in einer spezifischen Spannung zur Praxis. Entstanden ursprünglich ist sie durch „feldfremdes Kapital“ (Bourdieu), nämlich zunächst durch die bzw. aus der Frauenbewegung, insbesondere engagierte Lehrerinnen und Sozialpädagoginnen, heute zudem durch Bezug auf eine Männergruppenszene, Schwulen-, Lesbenbewegung und Queer politics. Diese Herkunft verlangt immer wieder einen gewissen Tribut. Gleichzeitig gibt sie der Forschung selbstverständlich auch normativen und motivationalen Rückhalt, ohne den wohl niemand in diesem karriereunfreundlichen Gebiet tätig wäre.

Eingebaut in den erfreulichen Prozess der Herausbildung einer ausdifferenzierten Lehre und Forschung sind also Spannungslagen, die mitbedacht gehören, will man die Lage der pädagogischen Frauen- und Geschlechterforschung beschreiben. Hierzu gehören selbstverständlich auch Fragen, die aus dem Umgang mit dem Geschlecht, der sexuellen Orientierung und Herkunft der Forschenden resultieren. Nun kann und soll an dieser Stelle keine Reflexion all dieser Spannungslagen geschehen. Die Diskussion der Forschung selbst soll im Zentrum stehen. Ihre Kontroversen wie ihre Leerstellen sind durchaus spannend genug.

Im Folgenden werde ich mich auf allgemeine bzw. bildungstheoretische Fragen konzentrieren. Es ist in der gebotenen Kürze hier nicht möglich, auf pädagogische Teildisziplinen einzugehen, auch wenn dies z.B. gegenüber dem entwickelten Forschungsstand in der historische Bildungsforschung, der Sozial- und Schulpädagogik ein verzerrtes Bild verursachen kann. Chronologisch werden zentrale Fragen und Paradigmen der pädagogischen Geschlechterforschung sowie die Männerforschung angesprochen und am Ende einige aktuellen Beobachtungen zur wissenschaftlichen Institutionalisierung berichtet bzw. zur Diskussion gestellt. 
Die Anfänge

Die ersten Themen der neueren pädagogische Frauenforschung ${ }^{3}$ (von einer Männerforschung kann noch nicht gesprochen werden, von einer Geschlechterforschung nur bedingt) können gruppiert werden um die Schlagwörter geschlechtsspezifische Sozialisation, Gewalt gegen Frauen und Mädchen und Sexismus in der Schule. Die Behandlung der Themen eint das Motiv einer Androzentrismuskritik pädagogischer Praxis und Theorie. Die folgenden Auseinandersetzungen in der pädagogischen Frauenforschung fragen entsprechend, wie Mädchen und Frauen angemessen in Erziehung und Bildung gefördert werden können. Hintergründe dieser anhaltenden Diskussionen sind die hundertjährige Debatte zur Koedukation, die kritische Bewertung der Bildungsreform, die feministische Debatte um Gleichheit oder/und Differenz und selbstverständlich die aktuelle Modernisierung der Geschlechterverhältnisse.

Eine Öffnung zu Fragen und Begriffen der Allgemeinen Pädagogik geschah erstmals durch die Konzepte Weiblicher Pädagogik Mitte der 1980er Jahre. Sie gehen über die Sozialisationstheorie und den Nachweis des Androzentrismus hinaus und bemühen sich auch normative Aussagen zu formulieren. Die Weibliche Pädagogik sieht in einer transformierten Weiblichkeit den Ansatz, die herrschende »männliche« Bildung zu überwinden. Theoretische wie praktisch orientierte Konzepte Weiblicher Bildung betonen die positiv erscheinenden Seiten von Weiblichkeit und ihren notwendig ergänzenden Charakter zu Männlichkeit.

Den Ansätzen einer Weiblichen Bildungstheorie z.B. von Hedwig Ortmann und Barbara Schaeffer-Hegel kann nun aber vorgeworfen werden, dass ihr Rekurs auf Weiblichkeit (und Natur) ungeklärt bleibt. Sie reformulieren implizit Grundlagen der Geschlechterordnung, da sie diese nur ansatzweise historisch und soziologisch aufklären. Vielfach fehlt ihnen so auch eine Selbstverortung der eigenen Normbegründung. Die bald einsetzende Kritik an der Weiblichen Pädagogik in der pädagogischen Frauenforschung grenzt sich entsprechend scharf $a b$.

3 Faulstich-Wieland (1995) setzt den Beginn der neueren pädagogischen Frauen- und Geschlechterforschung mit der ersten Berliner Sommeruniversität für Frauen im Juli 1976 an, die sicher eine besondere Ausstrahlungskraft besaß. Die Sommeruniversitäten wie die Vielzahl dezentraler Aktivitäten und Projekte gehen insgesamt auf die neue Frauenbewegung zurück, die sich spätestens seit den Frankfurter Tomatenwürfen vom September 1968 bundesweit zu organisieren begann. Im Herbst 1981 wurde die Arbeitsgemeinschaft Frauen und Schule gegründet. Nachdem seit der DGfE-Tagung von 1982 entsprechende Veranstaltungen organisiert worden waren, wurde 1985 eine Arbeitsgruppe Frauenforschung in der DGfE zugelassen, die heute zur Sektion aufgestiegen ist. 
Aus der Kritik der Weiblichen Pädagogik und der Kritik androzentrischer Erziehung kommt es aber zu einer Differenzierung der Androzentrismuskritik, ihrer erkenntniskritischen Grundlagen, pädagogisch-praktischen Folgerungen und der erziehungswissenschaftlichen Geschlechtertheorie. Dieser Prozess hält bis heute an. Es hat sich meines Erachtens gezeigt, dass dazu die feministische Theoriebildung ihre Grenzen überschreiten muss. So sollte, nach Christoph Türcke, zum Begriff der Vernunft und seiner ,männlichen“ Besetzung in der Geschichte vorgedrungen werden. Der Begriff erweist sich dabei einerseits als problematisch, weil männlich besetzt (siehe Gottvater oder bei Aristoteles), andererseits scheint seine Problematisierung ohne Vernunft nicht möglich. ${ }^{4}$ Weiter steht mit Brigitte Schmidt der Begriff des allgemeinen Subjekts in Frage, das verdächtige geschlechtliche Besetzungen aufweist, sodass auch emanzipatorische Bildungskonzepte, wie das von Heydorn, auf ihren Geschlechterbias befragt werden müssen. ${ }^{5}$

Als prominentester Ansatz der pädagogischen Frauenforschung Anfang der 1990er kann Annedore Prengels Buch Pädagogik der Vielfalt gelten. Es versucht in der Debatte um Gleichheit oder Differenz eine Vermittlung, übergeht aber die Texte von Türcke und Schmidt. Prengel kritisiert die Gleichheitsposition als Angleichung bzw. Defizitpädagogik; und die Differenzposition als latente Fortschreibung der Geschlechterhierarchie. Gegen den ,abendländischen Monismus“ begründet sie eine Position der „,egalitären Differenz“. Diese plädiert für die Gleichberechtigung vielfältiger Lebensweisen und -erfahrungen. Als ein zentrales Problem zeigt sich aber meines Erachtens auch bei Prengel, dass eine Vermittlung abstrakter, relationaler Bestimmungen von Gleichheit oder Differenz bzw. Gleichheit und Differenz ohne nähere Bestimmung der modernen Geschlechterverhältnisse unzureichend bleibt.

Den Abbruch der Debatte um Gleichheit und/oder Differenz, wie er dann in den 1990er Jahren stattfand, halte ich so für unbefriedigend. ${ }^{6}$ Ich denke, dass insbesondere die Fragen nach der »männlichen« Besetzung von Vernunft und (deskriptiver wie präskriptiver) Subjektivität erneut aufgegriffen werden sollten, so die Thesen von Türcke und Schmidt. ${ }^{7}$ Diese Fragen sind durchaus mit den aktuellen Diskussionen verwandt, sodass ich Heike Kahlert zustimme, die schreibt: „Nur scheinbar ist die Kontroverse um feministische Emanzipationsstrategien beigelegt, die vereinfacht unter den Etiketten Differenz und Gleichheit geführt wird“" (Kahlert 1995, S. 5).

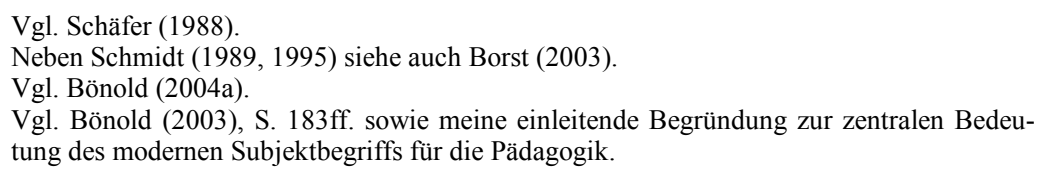




\section{Verunsicherung zu Beginn der 1990er Jahre}

Ab Anfang der 1990er Jahre gibt es eine Verunsicherung und Umorientierung in der pädagogischen Frauenforschung - ,mehrere Positionen streiten heftig miteinander" (Prengel 1995a, S. 138). Hintergrund für diesen Einschnitt ist, dass es im Feminismus mit den Debatten um Judith Butler ${ }^{8}$ und ethnometho-

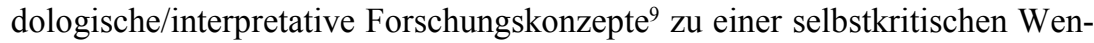
dung kam und, insbesondere für Deutschland, sich die theoretischen Bezüge ausweiten. Barbara Rendtorff spricht von „eine Art Paradigmenwechsel innerhalb der feministischen Theorie“ (Rendtorff 1998, S. 29), der eine neue Matrix an diskutierten Begriffen hervorbringt, die aber zunächst „nur mit Mühe verstehbar" (Prengel 1995a, S. 130) waren.

Die Pädagogik der Vielfalt hatte beispielsweise Geschlechtsunterschiede und Differenzen überhaupt begründet aus sozio-kulturellen Erfahrungen. Dies unterscheidet Prengel und andere ${ }^{10}$ grundsätzlich von einem sprachphilosophischen Differenzbegriff, wie ihn beispielsweise Rendtorff (1998) in der Tradition des Poststrukturalismus ${ }^{11}$ vertritt. Prengel geht davon aus: „Differenz bezeichnet gesellschaftliche Verschiedenheit, also unterschiedliche Lebensweisen und unterschiedliche Verarbeitung von Lebenserfahrungen“ (1990, S. 41). Dagegen schlagen Rendtorff/Moser vor, „Differenz als Spaltung, als das Offene, Nicht-Identische und Unabgeschlossene" (Rendtorff/Moser 1999, S. 315) zu beschreiben. „Differenz ist eine Bedingung der symbolischen Ordnung, welche durch eine Abwesenheit, einen ,Mangel` konstituiert ist" (ebd.).

Zwar holt sich die pädagogische Frauenforschung schon früh Anregungen aus dem Poststrukturalismus, so auch Prengel bei Irigaray und Lyotard bzw. Welsch. Prengel argumentiert allerdings selbst nicht poststrukturalistisch. Sie wendet sich gegen den Monismus des Abendlandes nicht sprachphilosophisch im Sinne des Aufzeigens der mangelhaften bzw. illusorischen Repräsentation der Welt durch Sprache - wie dies (verkürzt gesagt) der Poststrukturalismus

8 Für die Pädagogik siehe Maurer (1996), Szemerédy (1996), Behm u.a. (1999), Smykalla (1999), Fritzsche u.a. (2001) und Heinrichs (2001). Rendtorff grenzt sich von Butler ab (vgl. in Breitenbach 2002, S. 51ff.).

9 Die Ethnomethodologie bzw. das interpretative Paradigma werden auch als Doing-gender oder konstruktivistische Ansätze bezeichnet. Sie sind zumeist mikrosoziologisch orientiert. Vgl. Dausien (1996), Friebertshäuser/Prengel (1997), Breidenstein/Kelle (1998) oder Felden (2003).

10 S.a. Metz-Glöckel (1994) oder Faulstich-Wieland (1995).

11 Der Ausdruck Poststrukturalismus bündelt verschiedene philosophische Bemühungen, die erst im Nachhinein (insbesondere in den USA) mit diesem Ausdruck belegt wurden. Vgl. Dosse (1999) und Münker/Roesler (2000). 
täte. Prengel kritisiert das Denken bzw. die Vernunft, die totalitär die Mannigfaltigkeit der Welt als eine einheitliche Wahrheit fixiere, obwohl Welt nach Prengel ausschließlich perspektivisch darzustellen sei. Nur so ist verständlich, dass Prengel den de-konstruktivistischen Ansätzen die Aufforderung entgegenhält: „Konstruieren wir bewußt!“ (Prengel 1995b, S. 22). Prengel übergeht die poststrukturalistische Frage der Repräsentation von Wirklichkeit und auch die Frage nach interaktiven (Selbst-) Zuschreibungen von Geschlecht. Sie bleibt in der Tradition der pädagogischen Debatte um Gleichheit oder/und Differenz kritisch auf die Aufklärung orientiert.

Kern der neueren Theorien ist der Angriff auf die bisherige Konzeption des Begriffs der Geschlechter(differenz) in der Frauenforschung. Prengel unterstellt diesen auf normativer Ebene, dass sie „die Befreiung vom Frau-Sein (und) die Eröffnung der Möglichkeit unendlich vieler Geschlechter" (Prengel 2000, S. 86) betreiben. Nach Prengel ist es aber nicht legitim, als einzige Utopie die Geschlechtslosigkeit bzw. den Wechsel und die Vervielfältigung der Geschlechter anzusehen. Für die „Forschung und Theoriebildung sowie die mit ihr verbundene politische und pädagogische Praxis“ sieht Prengel „einen tiefen Einschnitt" (ebd.), der die Grundkategorie der Frauenforschung und damit sich selbst in Frage stellt. Sie sieht die Gefahr, dass es für die neuen Ansätze „Weiblichkeit eigentlich gar nicht mehr gibt“ (ebd.) und diese so politisch und pädagogisch nicht mehr handlungsfähig seien. Gegen diese Gefahr plädiert Prengel in der Forschung dafür, dass sich die Vielzahl an perspektivischen Zugängen gegenseitig anerkennen. Politisch bzw. pädagogischpraktisch plädiert Prengel für die Anerkennung verschiedener Emanzipationskonzepte. So sieht sie es z.B. als Verdienst der neueren Theorien an, Denkbeschränkungen aufgehoben zu haben, sowie dass die „Eigentätigkeit und Freiheit im Sozialisationsprozeß ... neu bewußt gemacht (wird).“ (Prengel 1995b, S. 19) Die Ansätze betonten allerdings einseitig die Freiheiten der Wahl. So ordnet sie die neuen Ansätze als „Postfeminismus“ in eine Perspektive der „Postmoderne“ ein. ${ }^{12}$

Prengels Kritik steht aus meiner Sicht exemplarisch für die oben angesprochene Verunsicherung der jungen pädagogischen Frauenforschung. Ihre Kritik soll hier nicht weiter untersucht werden, da hierzu verschiedenste Vorklärungen erforderlich wären. Hervorzuheben ist allerdings, was Prengels Kritik nicht leistet. Sie schätzt die neueren Ansätze ein, ohne deren verschiedene Grundbegriffe und Bezugstheorien kritisch darzustellen: Dekonstruktion, Poststrukturalismus, Ethnomethodologie, symbolischer Interaktionismus, Systemtheorie, ... Eine gegenseitige immanente Kritik der Positionen steht

12 Vgl. Prengel (1995a, S. 135ff.) und (2000). 
nach meiner Beobachtung bisher auch weiterhin aus. $\mathrm{Zu}$ anderen Klärungen ist es allerdings in der Zwischenzeit gekommen.

\section{Postmoderne?}

Das neue, unübersichtlichere Terrain der Debatte wird vielfach, nicht nur von Prengel, mit dem Begriff der Postmoderne verbunden. In der Zwischenzeit hat sich aber nach meiner Wahrnehmung gezeigt, dass der schillernde Begriff wenig zur Klärung beiträgt, ja durch seine verschiedenen Verwendungsweisen sogar die theoretische Debatte vernebelt. ${ }^{13}$

Postmoderne ist kein präziser Begriff, sondern, wie Marotzki schreibt, ein „Topos mit Such- und Verweisungscharakter“ (Marotzki 1992, S. 193). Der Begriff fängt verschiedene Fäden der Diskussion ein, ohne sie allerdings zu bündeln und weiterzuführen. Ich halte ihn für einen catch-all Begriff. Trotzdem greift er etwas auf. In der Postmoderne-Debatte treffen aus meiner Sicht verschiedene Denktraditionen vor dem Hintergrund traumatischer historischer und aktueller Ereignisse aufeinander. ${ }^{14}$ Daher auch seine Verwendung als Epochen-Begriff und seine Nähe zu Ausdrücken wie: Wissensgesellschaft, Individualisierung, Pluralisierung, Globalisierung, Kasinokapitalismus, Neue Medien, Kunst als Gegenmodell, Ästhetisierung des Denkens, Werterelativismus und -verlust, kulturelle Differenz u.a. Die Implikationen und Verzweigungen der Diskussion sowie ihre gesellschaftlichen Wurzeln können hier aber nur als Problem benannt werden. Es sollen im Folgenden nur wichtige Bezugspunkte der aktuellen pädagogischen Frauenforschung herausgestellt werden.

Für die bisherige pädagogische Frauenforschung gab (bzw. gibt) es zwei Anknüpfungsweisen zu(r) Postmoderne. Zuerst wird Postmoderne als Zeitdiagnose aufgefasst, ohne daraus begriffliche Konsequenzen zu ziehen. Es werden soziologische Begriffe und sozialwissenschaftliche Empirie betont. Befunde der Heterogenität der Lebensweisen, der Multikulturalität, des Wertere-

13 Ich denke, dass es notwendig ist, sich den poststrukturalistischen Geschlechtertheorien direkt und ohne Umweg über den Postmoderne-Begriff zu nähern. Vgl. Bönold (2003), S. $233 \mathrm{ff}$.

14 M.E. sind diese historischen Bedingungen auch für ein tieferes Verständnis des Poststrukturalismus nachzuvollziehen. Dies nicht deshalb, um die Theorien vorschnell als Mode zu kennzeichnen, sondern um ihre Ansprüche, Beweggründe und Fragen besser einschätzen zu können. Gerade der Poststrukturalismus fordert dazu auf, Theorieproduktion im Kontext zu betrachten. 
lativismus, oder der Patchwork-Identität stehen im Vordergrund und werden als neue „Herausforderungen“ (vgl. Krüger 1997, S. 245ff.) für die (Geschlechter-) Pädagogik gelesen. Fragen zur Begrifflichkeit und Methode werden als sekundär behandelt.

Ein Beispiel für dieses erste Anknüpfen ist das Buch von Birgit Warzecha: Gewalt zwischen Generationen und Geschlechtern in der Postmoderne (1995). ${ }^{15}$ Ausgehend vom postmodern anmutenden Thema der Medien thematisiert sie andere, die weniger postmodern klingen: Gewalt und die kapitalistische Ökonomie als konstituierende Merkmale derzeitiger Vergesellschaftung. Warzecha weist auf den gesellschaftlichen Wandel und seine Probleme hin, die hinter dem Schlagwort Postmoderne stehen. Das Verhältnis der Generationen und Geschlechter verändert sich. Massenmedien, Individualisierung und Wertepluralismus bedeuten lebenspraktische Herausforderungen für die Erziehung - nicht allein Provokationen an die erziehungswissenschaftliche Theorie. Warzechas Analyse plädiert für eine Pädagogik, die eine kritische Orientierung bietet. Pädagogik solle eindeutig „Position beziehen gegen Gewaltverhältnisse" (Warzecha 1995, S. 161).

Eine zweite Weise, an die Fragen der Postmoderne-Debatten anzuknüpfen, zielt mehr auf deren philosophische Dimension. Nach Maria Wolfs Übersicht über die Feministische Erziehungswissenschaft beispielsweise „befindet sich feministische Wissenschaftskritik in Übereinstimmung mit wissenschaftlichen Tendenzen des postmodernen Denkens" (Wolf 1996, S. 336). Wolf benennt drei weitere verwandte Fragen. Erstens die Frage nach den historischen Bedingungen: Die „Analyse und Kritik des binären Denkens, das ein hierarchisches Verhältnis zwischen den Gegensatzpaaren konstituiert, lassen sich auf den herrschaftlich strukturierten Dualismus von männlich und weiblich beziehen" (Wolf 1996, S. 338). Zweitens die Frage nach dem Status des Subjektbegriffs: „Die intendierte Überwindung der Subjektfixierung verspricht eine Befreiung aus den Zwängen der Geschlechtsidentität“ (ebd.). Und drittens sieht Wolf den Poststrukturalismus ,als Bezugspunkt feministischer Selbstkritik bei der Aufarbeitung der eigenen Befreiungspolitik" (Wolf 1996, S. 339) als hilfreich an. Damit spricht Wolf die Frage der eigenen Prämissen einer geschlechterpädagogischen Theorie und Praxis an sowie ihrer Zukunft.

Wolf legt hier aber eher ein Programm als ein ausgearbeitetes Ergebnis vor. Weiterhin sind zentrale Begriffe nur angedeutet und vielfach ausdeutbar. Sie zu klären erscheint nicht nur spannend, sondern auch notwendig, wenn man beispielsweise zurückdenkt, dass schon Hedwig Ortmann Formulierun-

15 S.a. Herwartz-Emden (1999) oder Kollers Bezug auf sozialwissenschaftliche Analysen: Koch/Koller (1999). 
gen von Prengel oder Rendtorff benutzt hatte, z.B. wenn sie von einer „Anerkennung von Differenz“ (Ortmann 1990, S. 23) schreibt oder: „Statt der geltenden Entweder-oder-Strukturen muß sich eine Denk- und Wahrnehmungsweise bilden, die das andere und die anderen als gleichwertig wahrnimmt. In ihr ist die Differenz der Erscheinungen möglich, ohne sie wertend zu spalten und hierarchisch zu ordnen" (Ortmann 1990, S. 15f.). Mehr begriffliche Schärfe erscheint zudem geboten, da es sich hier um relationale Aussagen (Gleichheit, Differenz, Gleichberechtigung, Anerkennung) handelt, die die Frage nach den Termen, zwischen den die Relationen bestehen (sollen), weitgehend undiskutiert lässt.

\section{De-/Konstruktion}

Aktuelle Debatten beziehen sich daher weniger auf den Postmoderne-Begriff als auf die verschiedenen Verwendungsweisen der Begriffe der Konstruktion bzw. Dekonstruktion von Geschlecht bzw. auf die dahinter stehenden Theorietraditionen. Die „De-Konstruktionsdebatte“ (Lemmermöhle 2000) begann in Erziehungswissenschaft allerdings verhältnismäßig spät. Dies hat notwendig zur Folge, dass die Theorien des Poststrukturalismus, des kognitionstheoretischen Radikal-/Konstruktivismus, des interpretativen Paradigmas, der Dekonstruktion usw. schon verschiedene Lesarten hervorgebracht haben und somit von vornherein eine unübersichtliche Situation vorliegt. Kahlert schreibt:

„Die Begriffe »Konstruktion« und »Dekonstruktion« gehören zu den wohl am häufigsten gebrauchten und zugleich irreführendsten Begriffen in den gegenwärtigen wissenschaftlichen Debatten zum Geschlecht. Konstruktion und Dekonstruktion werden in dieser Begriffsinflation umstandslos in eins gesetzt und oft losgelöst von ihren ursprünglichen Theoriekontexten“" (Kahlert 2000, S. 20).

Dazu kommt die pädagogische Sicht auf die Theorien:

„In der erziehungswissenschaftlichen Rezeption sind diese Diskurse weiteren Brechungen unterworfen. Einzelne ForscherInnen nehmen auf die genannten Diskurse je unterschiedlichen Bezug. Dabei kommt es auch zu Diskurs-Mixturen philosophischer und sozialwissenschaftlicher Bezüge. Diese Diskus-Mixturen kommen nicht zustande, weil die Erziehungswissenschaft die Differenzen der Diskurse ungenügend zur Kenntnis nimmt, sondern weil mit der Frage nach dem »Subjekt« aus erziehungswissenschaftlicher Perspektive die zentrale anthropologische Frage nach dem impliziten oder expliziten Menschenbild verbunden ist. Die erziehungswissenschaftliche Frauen- und Geschlechterforschung hat zu diesem Problemkreis eine besondere Affinität. Im Zusammenhang mit der Frage nach der 
möglichen »Konstruktion des Subjekts« bzw. dessen Dekonstruktion steht die Frage nach der möglichen Bedeutung der Kategorie Geschlecht: Ist die Kategorie »Geschlecht « für das Subjekt kardinal oder marginal?" (Klika 2000, S. 14)

Die Frage nach dem Subjekt und seiner geschlechtlichen Bestimmung tritt nach Klika in unterschiedlicher Gestalt in den Fokus. Der sozialwissenschaftliche (= interaktionstheoretische und kognitionstheoretische) Konstruktivismus betont ,den subjektbezogenen Charakter menschlicher Wahrnehmung und Erkenntnis“ (Klika 2000, S. 13). Der Poststrukturalismus hat das Subjekt anders im Blick: „Das Konzept des einheitlichen rationalen autonomen Subjekts wird de-konstruiert, als Konstruktion entlarvt“" (Klika 2000, S. 10). Rendtorff (2000a) arbeitet diesen Unterschied noch weiter heraus und fasst zusammen,

„daß man mit einer konstruktivistischen Perspektive das Geworden-Sein betont, die performative Herstellung, die Eigenaktivität der Individuen, die Konstruktionsleistung des Subjekts im Erkenntnisprozeß; und daß wir dekonstruktivistische Ansätze verwenden, um die Selbstgewißheit des Subjekts anzuzweifeln, um das Funktionieren, die subtilen und verborgenen Wirkungsweisen des Denkens offenzulegen, seine Machtgebundenheiten usw. und damit auch die Veränderlichkeit der Geschlechterordnung. Es ließe sich sogar die These vertreten, dass konstruktivistische Ansätze mit der Betonung der Konstruktionsleistungen der Subjekte und der Analysierbarkeit der Konstruktionsbedingungen gerade denjenigen Aspekt betonen und wertschätzen, den Dekonstruktion ihnen wieder unter den Füßen wegzieht, indem sie ihrerseits die »Technik«, den Akt des Konstruierens selbst auf seine festlegenden und ausschließenden Aspekte hin befragt und betont, daß Widersprüchlichkeiten, Heterogenitäten sich nicht in Klarheiten auflösen, sondern nur in einem »Gewebe von Spuren« darstellen lassen“" (Rendtorff 2000a, S. 47).

Auch wenn derartige (notwendig?) fehlenden Gewissheiten bis heute verunsichernd wirken, kann doch festgehalten werden, dass eine Klärung der Theorien und ihrer Fruchtbarkeit begonnen hat. Hinzuweisen ist hier auf die Arbeiten von Rendtorff ${ }^{16}$, Hartmann (2002), Borst (2003), Felden (2003) oder Bönold (2003). ${ }^{17}$ Der pädagogische Blick auf Geschlechtlichkeiten erweitert sich

16 Rendtorff bezieht sich auf die Subjektkonzeption/-kritik Lacans und auf Derridas »Begriff« der Différance. Die grundsätzliche Gespaltenheit und Geschlechtlichkeit des Subjekts lassen Bildung so notwendig offen und ohne Festlegung und Wahrheit erscheinen. Zu weitergehenden normativen Aussagen gelangt Rendtorff daher nur bedingt: vgl. (1998), S. 191f. oder (1999), S. 182. „Die Konzeption der Geschlechterverhältnisse“ steht nach Rendtorff dafür, ,wie eine Gesellschaft ihre Vorstellungen von Differenz, Abweichung, Normalität formuliert und dementsprechend ihre gesellschaftlichen Institutionen bildet.“ (1998, S. 31.) Bestimmungen dieser Vorstellungen und Institutionen sind bei Rendtorff m.E. aber bisher nur angedeutet und ihre Texte zentrieren vielfach in einer Sozialisationsperspektive. In ihren neueren Texten beginnt Rendtorff (2000a, b), ihre Subjektkonzeption mit den Fragen der allgemeinen Pädagogik zu verbinden

17 S.a. Maurer (1996), Szemerédy (1996), Behm u.a. (1999), Smykalla (1999), Fritzsche u.a. (2001) und Heinrichs (2001). 
so zusehend. Das (post-)moderne Geschlechterverhältnis und seine Beziehungen zu weiteren gesellschaftlichen Fragen und erkenntnistheoretischen Problemen wird weiter zu thematisieren sein. Die pädagogische Frauen- und Geschlechterforschung steht so heute vor der Aufgabe, einerseits die eigene, noch sehr junge Theoriegeschichte nicht zu vergessen und kritisch zu reflektieren. Andererseits steht sie vor den Herausforderungen der Postmoderne und DeKonstruktion.

\section{Pädagogische Männerforschung}

Auch wenn die pädagogische Männerforschung wenig zu den allgemeinen Debatten beigetragen hat, soll diese hier nicht unerwähnt bleiben. Als Forschung von Männern über Männer liegt ihr, bei aller Heterogenität der Ansätze, bisher ein latenter Differenzansatz, im Sinne der Annahme genereller Geschlechterdifferenzen, zugrunde..$^{18}$ Erst neuere Arbeiten, die sich z.B. auf Robert Connell oder den Poststrukturalismus beziehen, überwinden dies langsam. Lange steckte die Forschung in Bezügen der 1980er Jahre fest, d.h. betonte einseitig Geschlechtsunterschiede und versuchte diese mittels Sozialisations- und Rollentheorie zu erklären. Dies resultierte m.E. aus dem starken Praxisbezug, der die theoretische Auseinandersetzungen vernachlässigte.

Die pädagogische Männerforschung gibt es ab Beginn der 1990er Jahre. Sie entwickelt sich aus einer Männergruppenszene heraus und bezieht sich auf eine zunehmend ausdifferenziertere Praxis. So hat sich eine Väterforschung etabliert und es liegen verschiedene Studien zu Männerberatung, Kriminalität, Gewalt, Neo-Faschismus und männlicher Opfererfahrung vor. Mit drei Büchern sollen nun einige Schlaglichter auf die pädagogische Männerforschung geworfen werden - wobei auch hier chronologisch allgemeinere Beiträge dargestellt werden, während wichtige Beiträge zu Einzelthemen auBen vor bleiben müssen. ${ }^{19}$

Den Beginn der pädagogischen Männerforschung markiert m.E. das Buch von Dieter Schnack und Rainer Neutzling Kleine Helden in Not. Jungen auf der Suche nach Männlichkeit. Seine zahlreichen Auflagen machen es wahrscheinlich zum bis heute wichtigsten Buch der Jungenarbeit - wenn nicht der

18 Es gibt, auch über die Erziehungswissenschaft hinaus, bisher kaum Männern, die die Geschlechterverhältnisse thematisieren; in der Regel werden nur Männer bzw. Männlichkeiten thematisiert. Vgl. Döge (1999) oder den Informationsdienst Switchboard.

19 Hinweisen möchte ich auf Brandes/Bullinger (1996), Fthenakis (1999), Lenz (2000), May (o.J.), Neubauer/Winter (2001), Stuve (2001), Forster (2004) und Matzner (2004) 
ganzen pädagogischen Männerforschung, da es letztlich Männlichkeit über den ganzen Lebenslauf thematisiert. Es ist zwar stark journalistisch gehalten, aber die pädagogische Herkunft ist unverkennbar. Nach Schnack/Neutzling leiden Jungen an einer Reihe an Zumutungen, die an sie ,als Jungen“ gestellt werden. Jungen sind quasi unterdrückt von Rollenerwartungen. Die Qualität des Buches liegt nicht im theoretischen Zugriff, sondern in seiner einfühlsamen und parteilich-engagierten Schilderung der Jungen.

Die zweite, größere Veröffentlichung kam von Lothar Böhnisch und Reinhard Winter: Männliche Sozialisation. Bewältigungsprobleme männlicher Geschlechtsidentität im Lebenslauf. Hier wird anthropologisch und psychoanalytisch begründet, dass Jungen bzw. Männer eine „Außenorientierung“ des Verhaltens und der Wahrnehmung besitzen; quasi eine erlernte Blockade einer Wahrnehmung des Inneren (z.B. Angst) vorliegt. Mit sozialwissenschaftlichen Ansätzen begründen die Autoren, dass dieses Verhalten heute dysfunktional wird, d.h., Männer lernen müssen ihr Mann-Sein ,anders ${ }^{6} \mathrm{zu}$ bewältigen, als z.B. mit Gewalt (bei Hilflosigkeit). Das Buch ist theoretisch anspruchsvoller als das von Schnack/Neutzling, bleibt aber, wie auch die späteren Beiträge von Berno Hoffmann, auf die Theorie geschlechtsspezifischer Sozialisation fixiert.

Als drittes Buch sei die Dissertation von Rainer Strotmann (1997) erwähnt, die beginnt die Frage nach Männlichkeit relational und im pädagogischen Kontext zu betrachten. Er untersucht die Männlichkeits- und Geschlechtervorstellungen bei Rousseau, Pestalozzi, dann bei Nohl und Weniger sowie schließlich beim Berliner AK Didaktik und dem sog. Hamburger Didaktik-Modell von Schulz. Obwohl die Arbeit ebenfalls theoretisch auf die 1980er Jahre (Rollentheorie) orientiert bleibt, knüpft Strotmann mehr als die beiden anderen Bücher an die Ergebnisse der pädagogischen Frauenforschung an.

Dies fehlt m.E. der übrigen Männerforschung bisher vielfach. Sie will sich anders mit Männern und Männlichkeit beschäftigen als dies die traditionelle Wissenschaft tut, betrachtet Männer oder Jungen aber oft isoliert. Ihr Thema sind die Veränderungen von Männlichkeiten angesichts der Modernisierung der Geschlechterverhältnisse seit Mitte des Jahrhunderts; in etwa auf die Formel zu bringen als: Männer und Jungen sind heute gezwungen, sich mit veränderten Anforderungen auseinander zu setzen und können dazu nicht ungebrochen auf die traditionellen Männlichkeiten ihrer Väter zurückgreifen. Diese zweifellos bisher vernachlässigte Perspektive wird aber begrenzt, weil der Übergang weitgehend unterbleibt zu einem größeren theoretischen Rahmen, der Männlichkeiten in Beziehung zu den Geschlechterverhältnissen und sonstigen Gesellschafts- oder Diskursstrukturen setzen könnte. 
$\mathrm{Zu}$ den Zielen der Männerforschung gehört daher bislang selten, den positiven Bezug auf Männlichkeit aufzugeben. Die Formulierungen lauten hier z.B.: „parteiischer Blick auf Jungen“ (Schnack/Neutzling), ,Suche nach einer lebendigen und verantwortungsvollen Form des Mann-Seins“ (Männerbüro Göttingen), „für ein anderes Mannsein, eine andere Würde des Mannes“ (Böhnisch/Winter) oder „eine tiefgreifende kritische Reflexion der Geschlechterrolle" (Strotmann). So ist die Frage offen, inwieweit Jungenarbeit und Männerbildung eine Modernisierung i.S. einer dynamischen Reproduktion der Geschlechterverhältnisse betreiben oder in der Lage sind, eine Kritik von aktuellen Männlichkeiten zu betreiben. ${ }^{20}$ Die wissenschaftstheoretische Reflexion und Begründung von Gegenstand und wissenschaftlichen Selbstverständnis stehen für die pädagogische Männerforschung so letztlich noch aus. Bevor dies nicht geschehen ist, wird auch nicht mit einer Institutionalisierung von pädagogischer Männerforschung zu rechnen sein. Aufgrund der starken Nachfrage aus der Praxis hat sie an Fachhochschulen allerdings bereits begonnen.

\section{Zur Institutionalisierung der pädagogischen Frauen- und Geschlechterforschung}

Abschließen möchte ich mit einigen Beobachtungen zum institutionellen Stand der Frauen- und Geschlechterforschung überhaupt und den sich daraus eventuell ergebenen Auswirkungen für die Erziehungswissenschaft. ${ }^{21}$

Derzeit scheint sich, entgegen den Intentionen Vieler, die Frauen- und Geschlechterforschung als eine eigenständiges Fach herauszubilden. Man könnte auch sagen, es findet eine Disziplin-Werdung statt. Dies zeigt sich insbesondere daran, dass trotz vielfacher Skepsis zunehmend eigene Studiengänge Gender-Studies entstehen. Diese Studiengänge (i.S. von inhaltlich aufeinander aufbauenden Lehreinheiten) gibt es nun schon an 17 (von 97) Universitäten/Gesamthochschulen. Man kann vielleicht sogar von einem Boom dieser Studiengänge sprechen: 15 von ihnen sind jünger als sieben Jahre. Auch neue Zentren für Genderforschung entstehen - zunehmend im Zusam-

20 Der positive Bezug von Männerforschern auf Männlichkeit kann sogar parallelisiert werden zu dem positiven Bezug der Männer aus Männergruppen auf ihr neues Mannsein, wie ihn Michael Meuser (1998) als Schutz der habituellen Sicherheit herausgearbeitet hat.

21 Ich beziehe mich dabei auf Faulstich-Wieland (1995, 2003), Hildebrandt/Macha (1997), Bock (2002) und CEWS (2003). 
menhang mit Gender Mainstreaming ${ }^{22}$ Prozessen: CEWS/Bonn, Genderkompetenzzentrum Berlin. Dagegen scheint es aus verschiedenen Gründen als zweifelhaft, ob die Zahl der derzeit ca. 100 Frauenforschungsprofessuren (Denomination für Lehr- und Forschungsbereich Frauen- und Geschlechterforschung) erhöht werden wird.

Diese Entwicklungen können nun verschieden erklärt bzw. beurteilt werden. Betrachtet man zudem die vielen neuen Mentoring-Programme, die Besetzung von Genderforschungsstellen auch von Männern, Gender Mainstreaming Initiativen an den Hochschulen u.a., so kann man die These wagen, dass wir uns einem Übergang von einer klientelbezogenen (Förderung einzelner Frauen) zu einer strukturbezogenen Förderpolitik befinden. Für die Erziehungswissenschaft ist damit nicht allein die Beteiligung an den neuen GenderStudiengängen, sondern auch die strukturbezogenen Förderstrategien von Interesse. Hier geht es nämlich um Prozesse des Lernens von Organisationen. Pädagogische Perspektiven auf das organisationale Lernen und die Unterstützung von Lernprozessen Einzelner werden zunehmend gefragt sein. ${ }^{23}$

Zweifellos gibt es auch berechtigte Bedenken gegen entsprechende Strategien, so auch gegen Gender Mainstreaming. ${ }^{24}$ Entgegen anders lautender Befürchtungen haben aber nach meiner Wahrnehmung die Herausbildung eines eigenständigen Faches sowie eine strukturelle Förderpolitik bisher nicht zu einer Entpolitisierung und Entmündigung der Frauenhochschulpolitik geführt. So kommt es z.B. zu keiner institutionellen Trennung von Genderforschung und Frauenförderung: Es gibt derzeit nur ein reines Forschungszentrum (Kiel), sonst befindet sich m.W. Gleichstellungspolitik und Förderung der Genderforschung unter einem Dach. Die Konkurrenz von Ansätzen in der Frauen- und Geschlechterforschung seit dem sog. Butler-Boom und dem Durchbrechen der sog. Rezeptionssperre der Doing-gender-Ansätze führt zwar zu einer Spannung, aber nicht zu den befürchteten Einbrüchen in der Frauenförderung. Bis hin zu einer Lehreinheit mit Schwerpunkt Queer Studies in Hamburg kann aus meiner Sicht sogar von einer Ausdifferenzierung gesprochen werden.

Insgesamt sieht es natürlich derzeit durch die knappen Kassen auch für die Frauen- und Geschlechterforschung nicht rosig aus. Die Professionalisierung von Gender Studien und Gleichstellungspolitik wird durch die allgemeine Hochschulpolitik aber nicht nur behindert, sondern auch produktiv heraus-

22 Aus der Flut der Papiere zu Gender Mainstreaming vgl. Döge (2001), Blickhäuser (2002), Frey (2003) und Bönold (2004b). Zu Gender Mainstreaming an Hochschulen siehe Kahlert (2003) und Blome u.a. (2004)

23 Vgl. Böttcher/Terhart (2004) und Pasero/Priddat (2004).

24 Einen Überblick über die Einwände bietet Stiegler (2003). 
gefordert, indem (strukturbezogen) Profilbildung, Innovation und Qualitätskriterien eingefordert werden.

\section{Literatur}

Behm, Britta L./Heinrichs, Gesa/Tiedemann, Holger (Hg.) (1999): Das Geschlecht der Bildung. Die Bildung der Geschlechter. Opladen

Blickhäuser, Angelika (2002): Beispiele zur Umsetzung von Geschlechterdemokratie und Gender Mainstreaming in Organisationen. Berlin

Blome, Eva u.a. (Hg.) (2004): Handbuch zur universitären Gleichstellungspolitik. Von der Frauenförderung zum Gendermanagement?, Wiesbaden

Bock, Ulla (2002): Zwanzig Jahre Institutionalisierung von Frauen- und Geschlechterforschung an deutschen Universitäten. In: Feministische Studien. H.1

Böhnisch, Lothar/Winter, Reinhard (1993): Männliche Sozialisation. Weinheim

Bönold, Fritjof (1993): Jungen in Kindertagesstätten. Eine explorative Interviewstudie in sechs Nürnberger Kindertagesstätten. Bamberg

Bönold, Fritjof (2003): Geschlecht Subjekt Erziehung. Zur Kritik und pädagogischen Bedeutung von Geschlechtlichkeit in der Moderne. Herbolzheim

Bönold, Fritjof (2004a): Die (un)abgeschlossene Debatte um Gleichheit oder/und Differenz in der pädagogischen Frauenforschung. In: Zeitschrift für Frauenforschung und Geschlechterforschung. H.1

Bönold, Fritjof (2004b): Noch einmal: Was ist „Gender Mainstreaming“"? Begriffliche Annäherung an eine Praxis. In: Neue Praxis

Borst, Eva (2003): Anerkennung der Anderen und das Problem des Unterschieds: Perspektiven einer kritischen Theorie der Bildung. Baltmannsweiler

Böttcher, Wolfgang/Terhart, Ewald (2004): Organisationstheorie in pädagogischen Feldern, Wiesbaden

Brandes, Holger/Bullinger, Hermann (1996): Handbuch Männerarbeit. Weinheim

Braun, Christina von/Stephan, Inge (Hg.) (2000): Gender Studien. Eine Einführung. Stuttgart

Breidenstein, Georg/Kelle, Helga (Hg.) (1998): Geschlechteralltag in der Schulklasse. Ethnographische Studien in der Schulklasse. Weinheim

Breitenbach, Eva u.a. (Hg.) (2002): Geschlechterforschung als Kritik. Bielefeld

Center of Excellence Women and Science (CEWS) (2003): Hochschulranking nach Gleichstellungsaspekten. Bonn

Dausien, Bettina (1999): „Geschlechtstypische Sozialisation“ - Konstruktiv(istisch)e Ideen zu Karriere und Kritik eines Konzepts. In: Dies. u.a. (Hg.): Erkenntnisprojekt Geschlecht. Feministische Perspektiven verwandeln Wissenschaft. Opladen

Dosse, François (1999): Geschichte des Strukturalismus. 2 Bde. Frankfurt/M. 
Drerup, Heiner (1997): Die neuere Koedukationsdebatte zwischen Wissenschaftsanspruch und politisch-praktischem Orientierungsbedürfnis. In: Zeitschrift für Pädagogik. H.6

Döge, Peter (1999): Männerforschung als Beitrag zur Geschlechterdemokratie. Ansätze kritischer Männerforschung im Überblick. Berlin (im Auftrag des BMfFSFJ)

Döge, Peter (Institut für anwendungsorientierte Innovations- und Zukunftsforschung) (2001): Gendermainstreaming als Modernisierung von Organisationen. Ein Leitfaden für Frauen und Männer. Berlin

Faulstich-Wieland, Hannelore (1995): Geschlecht und Erziehung. Grundlagen des pädagogischen Umgangs mit Mädchen und Jungen. Darmstadt

Faulstich-Wieland, Hannelore (1998): Wissenschaft ohne Orientierung? Zu Heiner Drerups Koedukationsrezeption. In: Zeitschrift für Pädagogik. H.2

Faulstich-Wieland, Hannelore (2003): Einführung in Gender-Studien. Opladen

Felden, Heide von (2003): Bildung und Geschlecht zwischen Moderne und Postmoderne. Zur Verknüpfung von Bildungs-, Biographie- und Genderforschung. Opladen

Fleßner, Heike/Kaiser, Astrid (2002): Erziehungswissenschaft/Pädagogik. In: Kroll, R.: Gender Studies Geschlechterforschung. Ansätze Personen Grundbegriffe. Stuttgart

Forster, Edgar (2004): Männlichkeitsrituale als Widerstandsrituale in Erziehung und Bildung. Zur Konstruktion des (kritischen) Subjekts im Männlichkeitsdiskurs. In: ZfE. Beiheft 2

Frey, Regina (2003): Gender im Mainstreaming. Geschlechtertheorie und -praxis in internationalen Diskursen. Frankfurt/M.

Friebertshäuser, Barbara/Prengel, Annedore (Hg.) (1997): Handbuch Qualitative Forschungsmethoden in der Erziehungswissenschaft. Weinheim

Friebertshäuser, Barbara/Jakob, Gisela/Klees-Möller, Renate (Hg.) (1997): Sozialpädagogik im Blick der Frauenforschung. Weinheim

Fritzsche, Bettina/Hartmann, Jutta/Schmidt, Andrea/Tervooren, Anja (Hg.) (2001): Dekonstruktive Pädagogik. Erziehungswissenschaftliche Debatten unter poststrukturalistischen Perspektiven. Opladen

Fthenakis, Wassilios E. (1999): Engagierte Vaterschaft. Die sanfte Revolution in der Familie. Opladen

Giesecke, Wiltrud (Hg.) (2001=: Handbuch der Frauenbildung. Opladen

Hartmann, Jutta (2002): Vielfältige Lebensweisen. Dynamisierung in der Triade Geschlecht-Sexualität-Lebensform. Kritisch-dekonstruktivistische Perspektiven für die Pädagogik. Opladen

Heinrichs, Gesa (2001): Bildung Identität Geschlecht. Eine (postfeministische) Einführung. Königstein

Herwartz-Emden, Leonie (1999): Öffentlichkeit, Multikulturalität, Geschlechterverhältnis. In: Gogolin, I./Krüger-Potratz, M./Meyer, M.A. (Hg.): Pluralität und Bildung. Opladen 
Hildebrandt, Karin/Macha, Hildegard (1997): Frauenforschung in den Erziehungswissenschaften in den neuen Bundesländern. Eine Dokumentation. In: Zeitschrift für Frauenforschung. H.4

Hopfner, Johanna/Leonhard, Hans-Walter (1996): Geschlechterdebatte. Eine Kritik. Bad Heilbrunn

Hoffmann, Berno (1997): Das sozialisierte Geschlecht. Eine Theorie der Geschlechtersozialisation. Opladen

Kahlert, Heike (1995): Demokratisierung des Geschlechts- und Geschlechtervertrags. Noch einmal: Differenz und Gleichheit. In: Zeitschrift für Frauenforschung. H.4

Kahlert, Heike (2000): Konstruktion und Dekonstruktion von Geschlecht. In: Lemmermöhle u.a. (2000)

Kahlert, Heike (2003): Gender Mainstreaming an Hochschulen. Anleitung zum qualitätsbewussten Handeln. Opladen

Kleinau, Elke/Mayer, Christine (Hg.) (1996): Erziehung und Bildung des weiblichen Geschlechts. Eine kommentierte Quellensammlung. 2 Bde. Weinheim

Kleinau, Elke/Opitz, Claudia (1996): Geschichte der Mädchen- und Frauenbildung. 2 Bände. Frankfurt/M.

Klika, Dorle (2000): Zur Einführung: Konturen divergenter Diskurse über die Kategorie Geschlecht. In: Lemmermöhle u.a. (2000)

Koch, Martina/Koller, Hans-Christoph (1999): Geschlechter im Widerstreit? Ein Streitgespräch über Bildung und Geschlechterverhältnisse. In: Behm/Heinrichs/ Tiedemann (1999)

Krüger, Heinz-Herrmann (1997): Einführung in Theorien und Methoden der Erziehungswissenschaft. Opladen

Lemmermöhle, Doris/Fischer, Dietlind/Klika, Dorle/Schlüter, Anne (Hg.) (2000): Lesarten des Geschlechts. Zur De-/Konstruktionsdebatte in der erziehungswissenschaftlichen Geschlechterforschung. Opladen

Lenz, Hans-Joachim (2000): Männliche Opfererfahrungen. Problemlagen und Hilfeansätze in der Männerberatung. Weinheim

Lummer, Beatrix/Kampshoff, Marita (Hg.) (2002): Chancengleichheit im Bildungswesen. Opladen

Marotzki, Winfried (1992): Grundlagenarbeit: Herausforderungen für Kritische Erziehungswissenschaft durch die Philosophie J.F.Lyotards. In: Ders./Sünker, H.: Kritische Erziehungswissenschaft - Moderne - Postmoderne. 2.Bd. Weinheim

Matzner, Michael (2004): Vaterschaft aus der Sicht von Vätern. Subjektive Vaterschaftskonzepte und die soziale Praxis der Vaterschaft. Wiesbaden

Maurer, Susanne (1996): Zwischen Zuschreibung und Selbstgestaltung. Feministische Identitätspolitiken im Kräftefeld von Kritik, Norm und Utopie. Tübingen

May, Michael: Prolegomena zu einer materialistischen Theorie der Geschlechtlichkeit. Versuch einer Grundlegung für die Pädagogik. Unveröff. Habil. Frankfurt/M. o.J. 
Metz-Göckel, Sigrid (1994): Zur Kritik der Geschlechterkategorie und Geschlechterbeziehungen in der Erziehungswissenschaft. In: Pollok, G./Heid, H. (Hg.): Von der Erziehungswissenschaft zur Pädagogik. Weinheim

Meuser, Michael (1998): Geschlecht und Männlichkeit. Soziologische Theorie und kulturelle Deutungsmuster. Opladen

Münker, Stefan/Roesler, Alexander (2000): Poststrukturalismus. München

Neubauer, Gunter/Winter, Reinhard (2001): Dies und das. Das Variablenmodell. Balanciertes Junge- und Mannsein als Grundlage für die praktische Arbeit mit Jungen. Tübingen

Nyssen, Elke/Schön, Bärbel (Hg.) (1995): Perspektiven für pädagogisches Handel. Eine Einführung in Erziehungswissenschaft und Schulpädagogik. Weinheim

Oubaid, Monika/Kaiser, Astrid (1986): Gibt es eine weibliche Pädagogik? In: Kaiser, A.: Deutsche Pädagoginnen der Gegenwart. Köln

Ortmann, Hedwig (1985): Plädoyer für eine feministische Lebenswissenschaft: Entwurf eines Programms. In: Zeitschrift für Pädagogik. 19. Beiheft

Ortmann, Hedwig (1990): Bildung geht von Frauen aus. Frankfurt/M.

Pasero, Ursula/Priddat, Birger (Hg.) (2004): Organisationen und Netzwerke. Der Fall Gender. Wiesbaden

Prengel, Annedore (1990): Gleichheit versus Differenz - Eine falsche Alternative im feministischen Diskurs. In: Gerhard, U. u.a. (Hg.): Differenz und Gleichheit. Menschenrechte haben (k)ein Geschlecht. Frankfurt/M.

Prengel, Annedore (1995a)2: Pädagogik der Vielfalt. Verschiedenheit und Gleichberechtigung in interkultureller, feministischer und integrativer Pädagogik. Opladen

Prengel, Annedore (1995b): Wer sagt, daß Mädchen Mädchen sind...? Forschen und Erziehen im Wissen um die Widersprüche der Geschlechterverhältnisse. In: Hempel, M. (Hg.): Verschieden und doch gleich. Schule und Geschlechterverhältnisse in Ost und West. Bad Heilbrunn

Prengel, Annedore (2000): Perspektivtheoretische Fragen an die (De-)Konstruktionsdebatte. In: Lemmermöhle u.a. (2000)

Preuss-Lausitz, Ulf (1991): Der Kaiserin neue Kleider? Fragen an die feministische Schulforschung. In: Päd Extra. H.12 (siehe auch die anschließenden Erwiderungen)

Priem, Karin (1999): Frauen- und Geschlechterforschung in der Themenauswahl der »Zeitschrift für Pädagogik«. In: Feministische Studien. H.2

Rendtorff, Barbara (1996): Geschlecht und symbolische Kastration. Über Körper, Matrix, Tod und Wissen. Königsstein

Rendtorff, Barbara (1998): Geschlecht und différance. Die Sexuierung des Wissens. Eine Einführung. Königsstein

Rendtorff, Barbara (2000a): Geschlecht und Subjekt. In: Lemmermöhle

Rendtorff, Barbara (2000b): Das sexuierte Subjekt und der Andere in der Erziehungswissenschaft. Anmerkungen zu der Frage, wie "Subjekt“" und „Differenz“ zusammengehören. In: Neue Sammlung. H.3 
Rendtorff, Barbara (2000c): Pädagogischer Bezug und Geschlechterverhältnis In: Pädagogische Rundschau. H.6

Rendtorff, Barbara/Moser, Vera (Hg.) (1999): Geschlecht und Geschlechterverhältnisse in der Erziehungswissenschaft. Eine Einführung. Opladen

Schaeffer-Hegel, Barbara (1988): Die verborgene Bildung der Frauen. Plädoyer für ein offensives Bildungskonzept. In: Franck, N./Nemitz, R./Uhrig, B. (Hg.): Schulperspektiven. Bildung und Erziehung. Argument Sonderband 148

Schäfer, Alfred (1988): Zur Kritik der weiblichen Pädagogik. Bericht einer Arbeitsgruppe. In: Zeitschrift für Pädagogik. 23. Beiheft

Schmidt, Brigitte (1989): Zur Geschlechterdifferenz in Konzeptionen der Bildungstheorie. In: Widersprüche. H.30

Schmidt, Brigitte (1995): Im Sog der Abstraktion. Blicke auf männliche Theoriebildung. In: Euler, P./Pongratz, L.A. (Hg.): Kritische Bildungstheorie. Weinheim

Schnack, Dieter/Neutzling, Rainer (1990): Kleine Helden in Not - Jungen auf der Suche nach Männlichkeit. Reinbek

Smykalla, Sandra (1999): Die Subjektkonstitution im poststrukturalistisch-feministischen Diskurs - eine Provokation für die Pädagogik? Göttingen (unveröff. Magistraarbeit)

Stiegler, Barbara (2003): Gender Mainstreaming. Postmoderner Schmusekurs oder geschlechterpolitische Chance? Argumente zur Diskussion. Bonn

Strotmann, Rainer (1997): Zur Konzeption und Tradierung der männlichen Geschlechtsrolle in der Erziehungswissenschaft. Eine Analyse am Beispiel erziehungswissenschaftlicher Fragen und didaktischer Anwendungstheorien. Frankfurt/M.

Stuve, Olaf (2001): „Queer Theory“ und Jungenarbeit - Versuch einer paradoxen Verbindung. In: Fritzsche u.a. (2001)

Szemerédy, Susanne (1996): Oh boy, it's a girl. Dekonstruktion/Kritik der Kategorie Geschlecht - eine Chance für feministische Soziale Arbeit mit mißbrauchten/ mißhandelten Mädchen? In: Psychologie und Gesellschaftskritik. H.4

Tenorth, Heinz-Elmar (1999): Zur Rezeption feministischer Theorie-Impulse in der Erziehungswissenschaft - Transformation und Eingemeindung. In: Braunmühl, C.v. (Hg.): Der blockierte Dialog. Berlin

Türcke, Christoph (1993)²: Sexus und Geist. Philosophie im Geschlechterkampf. Frankfurt/M.

Warzecha, Birgit (1995): Gewalt zwischen Generationen und Geschlechtern in der Postmoderne. Eine Herausforderung für die Erziehungswissenschaft. Frankfurt/M.

Wolf, Maria (1996) ${ }^{4}$ : Feministische Erziehungswissenschaft. In: Hierdeis, H./Hug, T.: Taschenbuch der Pädagogik. Baltmannsweiler, Bd. 2 



\title{
Gedächtnisspeicher gesellschaftlicher Erfahrung? Zur politischen Dimension von Frauen- und Geschlechterforschung
}

\author{
Susanne Maurer
}

Im Sommersemester des Jahres 2004 wurde an der Universität Tübingen ein nicht nur für die Bildungsgeschichte denkwürdiges Jubiläum gefeiert: „100 Jahre Frauenstudium“. Wie schon an anderen Hochschulen zuvor sollte das Faktum des in mühevoller Arbeit und langjährigen Auseinandersetzungen von Frauen erkämpften Zugangs zur Hochschule gewürdigt werden, hier nun mit einer Ausstellung in der Universitätsbibliothek, mit einer Ringvorlesung im Studium Generale als klassisch-akademischer Form, mit vielen dezentralen Veranstaltungen in Fächern und Fachbereichen sowie etlichen Gesprächsrunden ,zwischen den Generationen“. In einer solchen Gesprächsrunde am Institut für Erziehungswissenschaft wurden auch die letzten 25 Jahre einer feministisch inspirierten pädagogischen Lehre und Forschung bilanziert - mit einem erstaunlichen Ergebnis und Effekt: Die ehemaligen Lehrenden und Studierenden auf dem Podium, Zeitzeuginnen einer durchaus wechselvollen ,Ära' der Entwicklung von Frauen- und Geschlechter- bis hin zur Männlichkeitsforschung, sahen sich einem Publikum gegenüber, das - staunend bis irritiert die Berichte und Gespräche verfolgte, und am Ende nur feststellen konnte, dass von all dem in der Gegenwart des Instituts und Studienalltags der meisten Studierenden nichts mehr zu spüren sei - daran ändert auch nichts, dass in der hauseigenen Bibliothek die Werke zum Themenkomplex im Laufe der Jahre viele Regale füllten. ${ }^{1}$

Auf was verweist diese Geschichte? Sie verweist meines Erachtens darauf, dass die Etablierung von Frauen- und Geschlechterforschung in der Hochschule keine einfache Erfolgsgeschichte ist. Sie verweist auch darauf, dass es ein Problem der Rezeption gibt, das sich auf erreichte theoretische Diskussionsstände ebenso bezieht wie auf den Stand der inzwischen vielfältig und breit vorliegenden empirischen Forschung. Dieses Problem der Rezeption

1 Später bildete sich aus diesem Erlebnis heraus eine Initiative von Studierenden mit dem Interesse, sich über Vortragsreihen wieder neu anzueignen, was es mit der Frage nach der Kategorie Geschlecht historisch und aktuell auf sich haben könnte. 
stellt sich nicht nur, aber auch im Generationenverhältnis. Die Geschichte verweist schließlich auf eine spezifische Qualität in der Entstehung und Entwicklung von Frauen- und Geschlechterforschung, die sich ihrer historischpolitischen Bedeutung im Kontext der Neuen Frauenbewegung verdankt und die ich mit so etwas wie „oppositioneller gesellschaftlicher Erfahrung ${ }^{“ 2}$ in $\mathrm{Zu}$ sammenhang bringen möchte.

Frauen- und Geschlechterforschung hat sich historisch als „Forschung und Theoriebildung in oppositioneller, gesellschaftskritischer Perspektive“ entwickelt, und hier soll nun danach gefragt werden, wie die Erfahrungen mit einer solchen Erkenntnispolitik produktiv weiter verarbeitet werden könn(t)en. Dies geschieht in drei Schritten: Zunächst wird, in einer Art systematisierendem Rückblick, die „Wissenschaftsfrage im Feminismus“ reflektiert (Abschnitt 1, „Dreiecks-Beziehungen ...“), sodann werden, in einer ganz bestimmten Facette, „Schwierige Tradierungen“ zum Thema gemacht (Abschnitt 2), um schließlich einen Denkvorschlag zu unterbreiten, der es erlaubt, die wie auch immer sich gestaltende Wirklichkeit der Frauen- und Geschlechterforschung als „Gedächtnisspeicher gesellschaftlicher Erfahrung“ aufzufassen (Abschnitt 3, „Abweichungsenergie erschöpft? ...“).

Der Beitrag argumentiert insgesamt für ein kritisch kultiviertes Bewusstsein von der (Gesellschafts-)Geschichte der Frauen- und Geschlechterforschung, die in spezifischer Weise auf Feminismus als transformative Politik bezogen ist. Werden die - inzwischen entfalteten - Denkmöglichkeiten zu „Geschlecht" von der Erinnerung an die damit jeweils verbundenen Kämpfe um eine Neugestaltung gesellschaftlicher Geschlechterverhältnisse abgespalten, so die damit verbundene These, so werden sie u.U. zu leicht instrumentalisierbaren Leerformeln, die ihr kritisches Potential und ihre politische Brisanz verlieren. Vor diesem Hintergrund erscheint die kritische Überlieferung der gesellschaftlichen Erfahrungen mit spezifischen (erkenntnis-)politischen Kämpfen als zentrales Moment einer feministisch inspirierten Forschung und Theoriebildung - soweit sie sich nach wie vor als Instrument der Transformation versteht.

2 Der Begriff ,gesellschaftliche Erfahrung“ wird hier als offenes Konzept genutzt, das sich je nach Thematisierungskontext - konkretisieren und präzisieren lässt. Gemeint sind zum einen individuelle Erfahrungen, die sich mit kollektiven Erfahrungen verschränken bzw. in den Kontext „Kollektivität“ eingebettet sind; zum anderen Erfahrungen, die mit Gesellschaft, der eigenen „Vergesellschaftung“" gemacht werden; zum dritten das Erleben, Wahrnehmen, Deuten zeitgenössischer gesellschaftlicher Prozesse und Situationen; und schließlich zeitgenössische Erfahrungen, die im Medium von „Gesellschaftsgeschichte“ und „Gesellschaftstheorie" thematisiert werden. In jedem Fall geht es also um Übergänge und Wechselbeziehungen zwischen Individualität und Kollektivität, was sich am Beispiel Sozialer Bewegungen deutlich zeigen lässt (vgl. etwa Maurer 1996). 


\section{Dreiecks-Beziehungen: Frauenbewegung, Feminismus und Frauenforschung}

Zunächst sollen einige Entwicklungslinien und Eckpunkte benannt werden, die das Kräftefeld „Frauenbewegung“ und „feministisch orientierte/inspirierte Wissenschaft" in der Bundesrepublik Deutschland kennzeichnen. Damit sind nicht zuletzt die „Anliegen der Frauenbewegung“"3 angesprochen - Wünsche, Hoffnungen, Visionen in Bezug auf eine ,geschlechtergerechte(re)“ Gesellschaft, damit konkret verbundene Bedürfnisse und Interessen, die historisch auch in die feministische Theoriebildung eingingen.

Der Begriff „Feminismus“ wird hier zunächst als Sammelbegriff verwendet, der den Komplex „Frauenbewegung“ ebenso umfasst wie den Komplex „feministisches Denken“. Sollen „Frauenbewegung“ und „Feminismus“ in ihrem Verhältnis zueinander bestimmt werden, so lässt sich erstere als soziale, politische und kulturelle Bewegung beschreiben, die mit dem feministischen Denken korrespondiert. Beide Komplexe hängen eng zusammen und werden deshalb manchmal auch synonym verwendet; die Grenzen zwischen ihnen sind durchlässig, und dennoch gehen sie nicht ineinander auf. Die Differenz lässt sich am ehesten so formulieren: „Bewegung“ kennzeichnet die Dimension der (auch leiblichen, auch energetischen) Erfahrung, die theoretische Perspektive „Feminismus“ strukturiert dabei das Feld der Wahrnehmung, das Interesse an und die Chance zur Erkenntnis.

Die Vielschichtigkeit des Komplexes Feminismus kann mit einer bestimmten Denkfigur konkreter gekennzeichnet werden, dem „Kräftefeld von KritikNorm-Utopie“. Demnach wäre Feminismus zum einen Kritik mit dekonstruktivem Potential (im Sinne der Kritischen Theorie, aber auch poststrukturalistischen Denkens ${ }^{4}$ ), zum anderen Utopie, mit (re)konstruktivem Potential und zum dritten Ethik mit normativer Kraft, die sich aus der Verbindung der beiden erstgenannten Dimensionen speist. Als Ethik oder normative Kraft erzeugt Feminismus Gebote und Verbote, d.h., auch „Moral“; als Utopie (er)schafft Feminismus Bilder - d.h. auch Dogmen über die „Wahrheit“", und als (Selbst-)Kritik ist Feminismus gleichzeitig in der Lage, Bilder und Dogmen (wieder) zu erschüttern. Erst auf dem Weg einer dogmenkritischen und selbstreflexiven Analyse „frauen-

3 Der Versuch gilt hier eher der systematischen Kennzeichnung, nicht der historischen Rekonstruktion, daher wird auch von „der Frauenbewegung“ und „dem Feminismus“ im Singular gesprochen.

4 Vgl. dazu ausführlich Maurer 1996, insbesondere S. 46-62. 
bewegter (Erkenntnis-) Praxen“"kann die Dynamik der verschiedenen Dimensionen im Verhältnis zueinander sichtbar werden. ${ }^{5}$

„Feminismus“ wird dabei in mehrfacher Hinsicht zur „gesellschaftlichen Erfahrung": indem feministisch inspirierte Lebensweisen und Lebensentwürfe (subjektiv, individuell) praktiziert werden und dabei eine gesellschaftliche Ausstrahlung entfalten; indem feministisch inspirierte Politiken, als bewegungsinterne wie -externe Praxis, in konkreten gesellschaftlichen und historischen Kontexten realisiert werden; indem Geschlechterverhältnisse analysiert und kritisch konzeptualisiert werden (Dimension der Theoriebildung).

Die Frauenbewegung wiederum hat sich als politische Bewegung herausgebildet, indem sie unwürdige Lebensverhältnisse und -bedingungen von Frauen öffentlich zum Skandal erklärte (Dimension der Kritik). Damit brach sie mit bestimmten gesellschaftlichen Tabus und setzte den bis dahin vorherrschenden Bildern von Weiblichkeit, von Frauen- und Mutterrolle provozierende andere Positionen entgegen (normative Dimension). Um die alten Bilder und Wirklichkeiten zu erschüttern, mussten zunächst radikal „andere“ Gegen-Entwürfe formuliert werden (Dimension der Utopie). Aber auch diese Gegen-Entwürfe blieben - notgedrungen - auf das bezogen, was bisher denkbar und lebbar war. Seit seinen Anfängen war (und ist) feministisches Denken daher von ganz bestimmten Spannungen oder Paradoxien durchzogen, die zum Beispiel darin bestehen, dass Frauen angesichts des tatsächlichen Ausmaßes sexistischer Gewalt zum einen als „Opfer“ von Männergewalt und Männerherrschaft erscheinen müssen, andererseits sich selbst aber auch als handelnde, schöpferische Menschen erfahren, die ihr Leben selbst gestalten.

Das Verhältnis von Feminismus und Wissenschaft wird im weiteren als Spannungsfeld mit drei Polen gekennzeichnet: „Feminismus als Bewegung“ ist darin zum einen bezogen auf Wissenschaft als Forschung und Theoriebildung sowie als Reflexion und analytische Durcharbeitung von Erfahrung, zum anderen auf Wissenschaft als spezifisches Gefüge von Disziplinen und Institutionen. Die „Wissenschaftsfrage im Feminismus“ bezieht sich also nicht nur auf die Institutionen und Disziplinen bzw. auf die theoretischen Systeme oder Horizonte. Es steht auch eine bestimmte Art des Umgangs mit „Wirklichkeit“ und Wirklichkeitserfahrungen zur Diskussion - ob nun innerhalb oder außerhalb der Universitäten.

Die Funktion und das Potential von Wissenschaft im Kontext einer gesellschaftskritischen Perspektive, im Interesse von ,politischen Minderheiten" ist bereits angeklungen: Forschung als Aufgreifen und Thematisieren

5 Eine solche Analyse habe ich in meiner Dissertation vorgenommen (vgl. Maurer 1996). 
des bislang Vernachlässigten und Unberücksichtigten, und Theoriebildung mit ,anderem Blick“ als Dekonstruktion des Herrschenden sowie als Reflexion und analytische Durcharbeitung von gesellschaftlicher Erfahrung. Damit dieses Potential sich in gesellschaftskritischer Perspektive aber auch längerfristig realisieren kann, müssen offenbar bewusst und systematisch immer wieder von neuem Bedingungen aufgesucht und geschaffen werden, die eine produktive Auseinandersetzung zwischen Wissenschaft und anderen Praxen ermöglichen.

Ein kritischer Einwand gegenüber ,wissenschaftlichen“ Betrachtungsweisen in der Perspektive politischer Praxis spricht immer wieder gar die reflexive Bewegung als solche an, die dann als Distanzierung von den anstehenden Aufgaben der Praxis, als Rückzug aus politischem Handeln wahrgenommen und interpretiert wird. Mit diesem Einwand wird eine durchaus problematische Erfahrung zum Ausdruck gebracht: dass wissenschaftliches Denken auf dem Weg der Analyse und Dekonstruktion einen Prozess der Differenzierung in Gang setzen kann, der auch die bisherigen Grundlagen des eigenen Selbstverständnisses radikal hinterfragt. So geschehen beispielsweise mit der Dekonstruktion der Kategorie „Frau“, die politisch erst einmal ,bewältigt“ werden muss(te).

Analytische Schärfe und Kraft, Differenzierung, Wahrnehmung und Herausarbeitung von Komplexität sowie Dekonstruktion von bisherigen Denkvoraussetzungen in befreiender Absicht tragen eben nicht automatisch zur Entwicklung - oder gar Erweiterung - von politischer und persönlicher Handlungsfähigkeit bei. Unter bestimmten Bedingungen können sie Handlungsfähigkeit - zumindest vorübergehend - auch einschränken oder gar blockieren: insbesondere wenn (wissenschaftlich orientierte) Denk-Bewegungen und sonstige politische, soziale und kulturelle Praxen stark arbeitsteilig organisiert sind und weder die Effekte der Praxis noch die Effekte der Theoriebildung mehr in einem geteilten - oder doch in Austausch stehenden - politischen Kontext weiterbearbeitet werden.

Dabei war (und ist) Feminismus notwendigerweise immer auch eine Denk-Bewegung: Mit der politischen und moralischen Kritik an bestehenden Ungleichheitsverhältnissen, die Frauen bestimmte Lebensmöglichkeiten verwehren, ging ein Nachdenken über das Warum und Wieso einher. Aus der politischen und moralischen Skandalisierung des Leidens und der Unterdrückung entwickelten sich gesellschaftstheoretische Analysen. Feministinnen erforschten Bedingungen und Begründungen der Ungleichheit und maßen letztere an gesellschaftlichen Werten und Prinzipien wie Gleichheit, Demokratie, Selbstbestimmung. In der Tradition marxistischer Kritik wurden Zusammenhänge offen gelegt zwischen einer Ökonomie, die auf spezifischen Verhältnis- 
sen der Arbeitsteilung beruht, und den Geschlechter-Macht-Beziehungen. Schließlich wurde immer mehr das Denken selbst zum Gegenstand der Untersuchung und der Kritik: Mit welchen Denkvoraussetzungen, mit welchen Denkoperationen konnten die „Wirklichkeiten von Frauen“ negiert und ausgeschlossen werden? Was machte die Arbeit, den Beitrag von Frauen zum gesellschaftlichen Leben unsichtbar? Und welche Konstruktionen, welche Bilder des Weiblichen und Männlichen hatten historisch dazu beigetragen, Frauen (und Männer) auf ihre gesellschaftlichen Plätze zu verweisen?

Frauen erforschten im Kontext der Frauenbewegung ihre eigenen Lebensbedingungen und Lebensgeschichten sowie die Geschichte/n der Frauengenerationen vor ihnen, um der historischen und sozialen Dimension weiblicher Existenz unter Bedingungen der Geschlechterhierarchie auf die Spur zu kommen. Sie suchten nach (gelebten oder lebbaren) Alternativen, nach Vor-Bildern in Vergangenheit und Gegenwart und entwarfen - literarisch, politisch und theoretisch - ein besseres Leben in der Zukunft.

Frauenbewegung hat also immer auch Frauenforschung beinhaltet. Feministisches Denken war auch feministisches Nachdenken und Forschen zu Geschlechterverhältnissen auf allen Ebenen: bezogen auf die Ebene des individuellen und kollektiven Handelns, auf die Ebene gesellschaftlicher Strukturen ebenso wie auf Prozesse der Strukturierung, bezogen auch auf die Ebene der Symbolischen Ordnung, des ,symbolischen Vorrats“ einer Gesellschaft.

Feminismus als Denk-Bewegung hat sich wissenschaftlicher Instrumentarien und Möglichkeiten bedient - ob innerhalb oder außerhalb der Universitäten. Nicht zuletzt diesem Umstand ist es zu verdanken, dass Feminismus eine differenzierte Wissenschaftskritik entfaltet hat - die bislang zur Verfügung stehenden Denkmöglichkeiten und Forschungsinstrumentarien hatten sich eben nur sehr begrenzt als geeignet erwiesen, Geschlechter(macht)verhältnisse und weibliche Lebensrealitäten in den Blick zu bekommen. Eine neue, andere Wahrnehmung musste entwickelt werden. Bereits in den frühen Jahren feministischer Theoriebildung war daher vom notwendigen ,anderen Blick“, von „Perspektivenwechsel“ die Rede.

Der ,andere Blick“ war das besondere und innovative Potential feministischen Denkens, mit dem es schließlich auch auf Achtung und Anerkennung innerhalb der akademischen Institutionen hoffen (und rechnen) konnte. Anders als bspw. in den USA hatte sich feministisches Denken in der (alten) BRD zunächst weitgehend außerhalb der etablierten Produktionsstätten von Wissenschaft entwickelt. Autonome Frauengruppen, als mehr oder weniger autodidaktische Diskussions- und Bildungszusammenhänge, waren der Kontext, von dem feministische Theoriebildung und Forschung ausging bzw. in 
dem sie sich ansiedelte und allmählich ausdifferenzierte, an Komplexität und analytischer Schärfe und Kraft gewann. ${ }^{6}$

Von Anfang an gab es allerdings auch einen Weg innerhalb der Universitäten: Studentinnen, vereinzelt auch lehrende Frauen trieben den Prozess sozusagen auf der ,Innenseite 'voran. Es bildeten sich Seminarkritik- oder Theoriegruppen in feministischer Perspektive, andere Inhalte und Fragestellungen wurden eingeklagt und ausprobiert, immer mehr feministisch orientierte Frauen qualifizierten sich auch formal im Rahmen der Universität und machten ihren Anspruch auf Teilhabe und Positionen geltend.

Der Weg in die Universität versprach den Zugang zu einem gesellschaftlichen Raum, der mit Status und Anerkennung versehen war. Dort die Stimme zu erheben, dort sichtbar zu werden, in dieser anderen Öffentlichkeit - davon erhofften sich Feministinnen nicht zuletzt die gesellschaftliche Anerkennung ihrer Anliegen. Beim Wechsel ins Feld der Universität mussten sich feministisch orientierte Forscherinnen allerdings auch mit einer Hierarchisierung von Wissensqualitäten und Erkenntnispraxen auseinandersetzen, die einen Teil ihrer politischen und persönlichen Erfahrungen entwertete. ${ }^{7}$ Es ist vielleicht verwegen anzunehmen, dass der sogenannte „Paradigmenwechsel" innerhalb der feministischen Theoriebildung - von manchen als Wechsel von „standpunktorientierten Epistemologien“ hin zu einem radikalen Konstruktivismus und zur Praxis der Dekonstruktion beschrieben - erkenntnispolitisch etwas mit dem Eintritt von Feministinnen ins Feld des Akademischen in seinen etablierten Gestalten zu tun haben könnte (vgl. zu dieser These List 1996). Ich möchte diese Denkmöglichkeit dennoch einbeziehen - im Kontext etwa einer möglichen zukünftigen Forschungsperspektive, mit der konkret, also historisch und empirisch untersucht werden müsste, welche spezifischen politischen, institutionellen, disziplinären und diskursiven Konstellationen von Frauen mitgeschaffen worden sind bzw. von ihnen genutzt werden konnten bzw. es ihnen ermöglicht haben, sich im Feld des Akademischen - gerade auch mit feministischen Erkenntnisinteressen - Anerkennung zu verschaffen.

6 Dass hier auch spezifische Probleme entstanden, soll nicht verschwiegen werden: so hatte das ,,wilde Denken“ außerhalb der Universität bspw. mit Problemen der Ungenauigkeit und der zu großen Themenbögen zu tun, im Forschungs- und Erkenntnisprozess wurde zum Teil auch Mythen- und Legendenbildung betrieben (Beispiel Hexenverfolgung) - zumindest letzteres trifft allerdings auch auf so manche, wissenschaftliche Praxis‘ innerhalb der Institutionen zu.

7 Maya Nadig und Mario Erdheim haben einmal beschrieben, wie „das Akademische“ lebendige Erfahrung zunichte machen kann. Sie gehen so weit, von der „Zerstörung wissenschaftlicher Erfahrung durch das akademische Milieu“ zu sprechen (vgl. Nadig/Erdheim 1984) 
Sind die „unorthodoxen Denker/innen“ mit ihren dekonstruierenden Verfahren damit womöglich einer Dynamik der Domestizierung ausgesetzt, die ein neues Regelwerk entfaltet, das mit hegemonialem Machtanspruch auftritt? Einen interessanten und provozierenden Beitrag zu dieser Frage leistet Tove Soiland (2004), indem sie aktuelle Gender-Theorien im deutschsprachigen Raum, insbesondere auch die Queer Studies, mit Michel Foucaults Gouvernementalitätsanalyse kritisch gegenliest (vgl. hierzu allgemein Bröckling u.a. 2000). Ihrer Ansicht nach bleiben viele der aktuellen - von Judith Butler u.a. inspirierten - Beiträge einem Machtbegriff verhaftet, der einen wichtigen Schritt im Denken Foucaults noch außer Acht lässt: die These nämlich, dass heute - im Kontext von Postfordismus oder Neoliberalismus - eine neue Form von „Menschenregierungskünsten“ im Spiel ist, die geradezu einen $\mathrm{Pa}$ radigmenwechsel in den Machttechnologien anzeigt (gemeint ist eine spezifische Verknüpfung von Fremdführung und Selbstführung im „Sicherheitsdispositiv“, vgl. etwa Foucault 1993). Einem Gedanken Foucaults zufolge besteht die Wirkung eines Dispositivs gerade darin, die Aufmerksamkeit bspw. Sozialer Bewegungen - auf die Machttechnologien der vorausgehenden Epoche zu lenken. Tove Soiland wendet diesen Gedanken nun auf die aktuellen Geschlechtertheorien an: „Glauben wir nicht ... dass man zur Macht nein sagt, indem man zu queer ja sagt; man folgt damit vielmehr dem Lauf des neoliberalen Flexibilisierungsparadigmas. (...) ... es stellt sich mir darüber hinaus die Frage, ob gender nicht gerade mit seiner dezidierten Einforderung von Gestaltungsmöglichkeiten des eigenen Selbst nunmehr seinerseits $B e$ standteil des gegenwärtigen Geschlechterregimes geworden ist" (Soiland 2004, S. 3).

Wie Soiland gehe ich davon aus, dass die „Dekonstruktion“ als erkenntnispolitische Strategie nicht einfach per se subversiv ist, sondern sich ebenso wie andere Strategien im Feld feministischer Denk-Bewegungen bestimmten historischen Konstellationen verdankt. ${ }^{8}$ Auch die aktuellsten GeschlechterTheorien müssen sich also mit der Frage konfrontieren lassen, inwiefern sie produktiver Bestandteil eines Machtfeldes sind, das geschlechterasymmetrische Effekte erzeugt.

Veränderungsprozesse im Sinne einer feministischen Perspektive erhalten ihre Komplexität dadurch, dass sie sich nicht nur auf die soziohistorische Situation, sondern gleichzeitig auch auf den Korpus des Wissens darüber bezie-

8 Mit Bezug auf Adkins (2004) stellt Soiland fest, dass ,die Bedingungen, unter denen überhaupt Identität zum Gegenstand politischer Aushandlung, theoretischer Reflexion oder persönlicher Verfüg- und Einsetzbarkeit wird (...) ihrerseits als strukturelle verstanden werden [müssen], als sich historischen und sozioökonomischen Voraussetzungen verdankend, die offenbar geschlechtlich codiert sind“" (Soiland 2005, S. 13). 
hen - von daher stellt sich hier immer auch die Frage nach der Möglichkeit einer emanzipatorischen Theoriepolitik (vgl. Ernst 1999): „Die Person dieser epistemischen Subjektposition hat keinen allumfassenden Blick, dafür eine soziokulturelle Geschichte, die sie zur Konstruktion ihrer Fragestellung führt, und ein Erkenntnisinteresse, das die Emanzipation aus gegebenen Positionierungen in Geschlechterhierarchien anstrebt" (Ernst 1999, S. 20).

In ihrer wissenschaftsphilosophischen Studie formuliert Waltraud Ernst einen Begriff von Veränderung, ,der sowohl auf der wissenschaftlichen Ebene als auch in der Dimension sozialer Wirklichkeit von historisch groß angelegten Umwälzungen absieht und statt dessen epistemische und soziale Veränderungen als stete, kontinuierliche Auseinandersetzungsprozesse versteht, die sich gegenseitig bedingen und produzieren“ (Ernst 1999, S. 11). Mir erscheint es für feministisches Denken und feministische Praxis bspw. ebenso wichtig, klassische Subjekt-Konzeptionen radikal zu hinterfragen, wie unabdingbar, auch für Frauen den Status eines Subjektes zu fordern und zu ermöglichen: eines Subjektes der Erkenntnis, der Politik und - nicht zuletzt - der eigenen Lebensgestaltung. Gemeint ist hier allerdings ein prozessuales, relationales, konstituiertes und radikal situiertes Subjekt, das sich immer wieder als solches hervorbringt bzw. erst hervorbringen muss (vgl. z.B. Kahlert 1996).

\section{Schwierige Tradierungen: GenerationenMachtBeziehungen zwischen Frauen im Wissenschaftsbetrieb}

Frauen treten sich als „Angehörige verschiedener Generationen“ im Rahmen der Hochschule auf durchaus unterschiedliche Weise gegenüber: in den verschiedenen Rollen als Lehrende und Studierende zum einen, als Wissenschaftlerinnen auf (zumindest formal) verschiedenen Qualifikationsniveaus zum anderen, und als Kolleginnen derselben Statusgruppe mit unterschiedlichen, generationstypischen' Erfahrungshintergründen zum dritten.

Jedes der - hier lediglich idealtypisch gekennzeichneten - Generationenverhältnisse ließe sich daraufhin untersuchen, wie die Erfahrung einer Generationendifferenz überhaupt zustande kommt und auf welcher Ebene diese Differenz zum Ausdruck gebracht - oder auch verkannt wird.

An einem fiktiven Szenario (mit realem Hintergrund) kann gezeigt werden, wie sich Generationenverhältnisse in problematischer Weise überkreuzen können, und damit nicht nur die Möglichkeit von Förderbeziehungen unter Frauen erschweren, sondern auch den Austausch von Erfahrungen mit unterschiedlichen erkenntnispolitischen Strategien: 
Eine Gruppe von feministisch orientierten Hochschullehrerinnen verschiedener Fachrichtungen institutionalisiert einen interdisziplinären Forschungszusammenhang, in dessen Rahmen auch Nachwuchswissenschaftlerinnen (auf dem Weg zur Promotion oder Habilitation) integriert und gefördert werden sollen. Die Praxis des „Du“ erscheint geradezu selbstverständlich, sowohl unter den Professorinnen selbst wie auch zwischen Professorinnen und dem weiblichen wissenschaftlichen Nachwuchs. ${ }^{9}$ Problematisch kann diese Praxis des „Du“ bspw. dann werden, wenn damit die Status- und Machtgefälle sowie die in den strukturellen Beziehungen durchaus unterschiedlichen Verantwortlichkeiten verwischt oder gar überdeckt werden.

Ich möchte hier die These formulieren (und reflektiere damit in gewisser Weise auch eine eigene Erfahrung), dass es für feministisch orientierte Hochschullehrerinnen nicht gerade einfach ist, ihre Position im Generationen(macht)gefüge - und damit auch im Gefüge möglicher Förderbeziehungen wirklich einzunehmen. Das hat sicherlich vielschichtige Gründe. Drei seien genannt: Erstens ist die Wirkung der nach wie vor als männerbündisch zu bezeichnenden Dynamiken und Praxen im deutschen Hochschulwesen nicht zu unterschätzen - ob Frauen im Kontext der Hochschule das nun wollen oder nicht, sie finden sich vor diesem Hintergrund, sozusagen ,homogenisiert', immer wieder in der Geschlechtsgruppe der „Frauen“ wieder. In dieser Perspektive erscheint selbst die etablierte Professorin gelegentlich als diskriminiert, marginalisiert und stigmatisiert. Zweitens trägt die - für viele der Älteren biographisch noch bedeutsame - spezifische Erfahrung mit kollektiver Praxis im Zusammenhang der Neuen Frauenbewegung dazu bei, dass Differenzen und Kontroversen sowie Machtgefälle unter Frauen nicht unbedingt offen, womöglich konfrontativ thematisiert, geschweige denn produktiv ausgetragen oder gar kultiviert werden (können).$^{10}$ Drittens erschwert auch die Geschichte radikaler feministischer Kritik an Institutionen im Allgemeinen und am Wissenschaftssystem im Besonderen für manche die Anerkennung der eigenen Teilhabe an den entsprechenden, strukturell mit Macht ausgestatteten Positionen. (Hier wäre die Frage nach gesellschaftstheoretischen und machtanalytischen Konzepten zu stellen, die es ermöglichen, die eigenen Bewegungen

9 Dieses Detail wird bewusst angeführt: Das „Du“ steht hier zum einen für die Geschichte der Bemühungen um eine Hochschulreform, die Geschichte der versuchten Demokratisierung und Enthierarchisierung der Universität. Zum anderen verweist es auf eine ,generationentypische“ Erfahrung von Kollektivität - im Kontext der antiautoritären Studentenbewegung bzw. der Frauenbewegung.

10 Den Hintergrund hierfür bildet ein historisch spezifisches Verständnis von „Solidarität auf der Basis von gleicher Betroffenheit", das trotz inzwischen vielfach vorgenommener selbstkritischer Analysen noch wirksam sein kann. 
als Akteurinnen im „strukturierten Feld der Universität“ auf angemessene Weise zu fassen.)

Für die am fiktiven Szenario beteiligten ,jüngeren“ Frauen, die sich in der strukturellen Position der Nachwuchswissenschaftlerin befinden, stellt sich die Situation anders dar. Sie fühlen sich unter Umständen in ihrer Erwartung und Hoffnung getäuscht, von den ,älteren“ - scheinbar ja etablierten Frauen auf klare und eindeutige Weise gefördert zu werden. Sie verkennen dabei den nach wie vor prekären Status ,ihrer' Professorinnen im System Universität. Was erschwert nun eine angemessene Wahrnehmung der gesamten Situation aus ihrer Sicht? Ich nenne wiederum beispielhaft einige Aspekte: Für viele jüngere Wissenschaftlerinnen scheint es inzwischen selbstverständlicher zu sein, sich für eine akademische Laufbahn zu interessieren. Viele Hindernisse scheinen aus dem Weg geräumt, „,weibliche Intellektualität“ hat den ,Geruch des Abnormen' verloren. Der eigene Lebensentwurf scheint ,freier' gewählt, mehr dem ,individuellen Management' überlassen. Dabei hat sich die gesellschaftliche und familiäre Arbeitsteilung zwischen Frauen und Männern statistisch gesehen kaum verändert. Aber es sind neue ,Leitbilder entstanden: von Frauen, die beruflich wie privat (sehr) erfolgreich sein können. (Die Erfahrung der Überlastung, die aus der Orientierung an solchen Leitbildern erwachsen kann, ist unter Umständen noch nicht gemacht.) Die ,alte', entwertete Frauenrolle scheint also von neuen Lebensmöglichkeiten abgelöst - und lebt doch weiter fort. Der damit angesprochene latente Geschlechterkonflikt ist gesellschaftlich jedoch nicht mehr so leicht thematisierbar (vgl. bspw. Bitzan 1996).

Aus der Perspektive der Jüngeren ,überkommene Feminismen ' werden so eher abgewehrt. Es hat sich ja tatsächlich einiges verändert; wie umkämpft und um welchen Preis diese Veränderungen zustande gekommen sind, entzieht sich allerdings immer wieder der Wahrnehmung und - fast systematisch - dem ,kollektiven Gedächtnis‘. Veränderungsprozesse werden manchmal außerdem vereindeutigt zu einer „Fortschritts- (oder Rückschritts-!)geschichte“ (zum Beispiel im Bereich des Theoretischen), die damit verbundenen Brüche und Widersprüche, die gegenläufigen Dynamiken geraten aus dem Blick und können daher auch nicht analytisch bearbeitet werden. Ein möglicher Effekt: Jüngere Frauen sehen den Geschlechterkonflikt konkret nicht unbedingt mehr als ihr Problem an, selbst wenn sie ihn theoretisch noch bearbeiten. Denkbar ist, dass hier auch der Wunsch und das Interesse eine Rolle spielen, eigene avantgardistische - Aufbrüche zu gestalten. (Die Attraktion der „queer studies" kann nicht zuletzt auch vor einem solchen Hintergrund gesehen werden.) 
Wenn hier von ,jüngeren“ und ,älteren“ Frauen die Rede ist, so unterliegt diese Rede selbst einer Dynamik, die hier eigentlich problematisiert werden soll: Die Rede von „Generationen“ bringt ja ebenfalls eine Vereindeutigung, eine Homogenisierung von Situationen und Erfahrungen mit sich: die Relativierung oder (Verdeckung) der Differenzen innerhalb ,einer Generation“, um andere Differenzen (zwischen „den Generationen“) beschreiben und thematisieren zu können. Aus diesem Dilemma kann eine nicht so ohne weiteres aussteigen, zumindest aber versuchen, es bewusst zu halten.

Im oben angedeuteten fiktiven Szenario des feministischen Forschungszusammenhangs spielen, um es noch einmal zusammenzufassen, unter Umständen also mehrere widersprüchliche Generationenverhältnisse eine Rolle: Die beteiligten Hochschullehrerinnen haben - selbst als Feministinnen - Anteil an der Macht der Institution (strukturelle und symbolische Macht). Diese Macht setzen sie ein, um eine neue Infrastruktur und potentielle Förderbeziehungen zu ermöglichen und auszugestalten. Die - Frauen implizit oder explizit nach wie vor marginalisierende - Dynamik der Institution errichtet den Machtmöglichkeiten der Professorinnen allerdings immer wieder spezifische Grenzen. Zugespitzt: Als Inhaberinnen ihrer Status-Position haben die Professorinnen Macht, als Angehörige der Geschlechtsgruppe „Frauen“ können sie sich situativ immer noch/immer wieder als „ohnmächtig“ erleben. Die Nachwuchswissenschaftlerinnen wiederum sind als Inhaberinnen ihrer StatusPosition von den Professorinnen und den Verfahren der Institution abhängig, also relativ „machtlos“, als Angehörige der Geschlechtsgruppe „Frauen“ erleben sie sich in der Regel nicht (mehr) als „ohnmächtig“, sondern eher als entscheidungsmächtige Individuen mit Verfügungsmacht über ihren eigenen Lebensentwurf.

Würden beide Gruppen ihre „Generationen-“(Status-)positionen im institutionellen Machtgefüge anerkennen und zu einem bedeutsamen Bezugspunkt ihres Handelns machen, so hieße das - immer idealtypisch gesprochen - für die „Älteren“ bspw., dass sie ihr früheres Selbstverständnis als „Rebellinnen des Aufbruchs" (das manche in der eigenen Lebensführung vielleicht noch kultivieren) verändern und sich selber auch als „etablierte Autorität“ wahrnehmen müssen; für die „Jüngeren“, dass sie die „Älteren“ in ihrer Autorität, ihrem Wissen, ihrer Kompetenz, und auch mit ihren (gesellschaftlichen) Erfahrungen wahrnehmen und anerkennen müssen.

In einem solchermaßen neu sich gestaltenden Generationen-Machtgefüge zwischen feministisch orientierten Frauen im Wissenschaftsbetrieb wären veränderte Tradierungsverhältnisse vorstellbar, die es meines Erachtens ermöglichen würden, frühere Denk-Bewegungen und -vorschläge nicht einfach etwa einer Fortschrittslogik folgend - zu verwerfen, sondern sie kritisch zu 
kontextualisieren und in ihrer erkenntnispolitischen Bedeutung zu würdigen sowie die damit verbundenen gesellschaftlichen Erfahrungen aufzugreifen und weiterzubearbeiten.

\section{Ist die Abweichungsenergie erschöpft? \\ Frauen- und Geschlechterforschung als „Gedächtnisspeicher in transformativer Absicht"}

Die eingangs erzählte Geschichte verweist auf ein ,ganz banales` Problem: Neue ,Normalitäten', die meist mühsam errungenen neuen ,Selbstverständlichkeiten' erzeugen oft den Effekt, dass die - zumindest in der Vergangenheit - zugrunde liegenden (sozialen) Konflikte kaum noch thematisierbar sind. Mit den Erfolgen der frauenbewegten, frauenpolitischen Praxis (bspw. dem erkämpften Zugang zum Hochschulstudium) verschwindet tendenziell ein gesellschaftlich geteiltes, kollektiv verfügbares Problembewusstsein in Bezug auf nach wie vor existierende strukturelle Ungleichheit.

Doch wie ließe sich die dem zugrunde liegende ,Erfolgsgeschichte“ überhaupt resümieren? Vielleicht so: Frauenbewegung und Feminismus haben neue Fragen gestellt und neue Zweifel angemeldet, haben neue Visionen in die Welt gesetzt und auch neue Setzungen vorgenommen, haben Wahrnehmungen und Erfahrungen von Frauen mit Bedeutung versehen - nicht zuletzt im Medium der Frauen- und Geschlechterforschung, die damit in ihrer gesellschaftspolitischen Bedeutung erkennbar wird.

Feministische Impulse und Initiativen waren und sind, gerade auch in der Pädagogik, innovativ wirksam, brachten und bringen Themen und Anliegen ins gesellschaftliche Spiel, entwickel(te)n eine neue Praxis und bilde(te)n dabei auch neue Strukturen aus. Mit der Kraft der Bewegung wurden alte Strukturen aufgebrochen, verändert, umgewandelt. Es entstand dabei auch eine gemeinsame gesellschaftliche Arbeit, deren soziale und kulturelle Dimension nicht zu unterschätzen ist: Beziehungsnetze sind entstanden und können auch heute noch politisch wie persönlich genutzt werden. Neue Lebensweisen sind erprobt worden, auf diese kollektiv geteilte Erfahrung können sich Frauen auch heute noch beziehen, sie evtl. in kritischer gemeinsamer Auseinandersetzung auswerten und weiterentwickeln.

Geschlechter-Bilder sind in Bewegung gebracht worden - auf allen Ebenen und im ganzen Spektrum der kulturellen Ausdrucksformen. Eine Verflüssigung der Geschlechtergrenzen hat stattgefunden, neue Lebensmöglichkeiten 
sind tatsächlich entstanden. Und doch bleibt dabei immer wieder von neuem und genau zu bestimmen: Was verschwindet aus der Wahrnehmung und gesellschaftspolitischen Thematisierbarkeit? An diese Frage erinnert nicht zuletzt der im ersten Abschnitt zitierte Beitrag von Tove Soiland.

Die amerikanische Theoretikerin Judith Lorber (1999) spricht von der Erosion und der gleichzeitig doch ungebrochenen Wirksamkeit von Geschlechterverhältnissen. Die ,automatische Geschlechtserkennung (und Geschlechts-Performanz) im Alltag“ (doing gender), wie sie insbesondere mit der Perspektive des Sozialen Konstruktivismus von Forscher(innen) sehr detailliert untersucht werden, stehen neben Versuchen des undoing gender - der Bagatellisierung, De-Thematisierung oder auch der Entdramatisierung von „Geschlecht“.

Im heute scheinbar so unbeschwerten Spiel mit den Geschlechter-Bildern findet doch immer wieder auch deren Beschwörung und Reproduktion statt. Momente der Subversion in befreiender Absicht, der spielerischen Aneignung in Freiheit sind davon oft kaum zu unterscheiden. Es bleibt das Problem der Gerechtigkeit angesichts der Realität sozialer Ungleichheit: der Kampf um Zugang, Ressourcen, Wahlmöglichkeiten und Unterstützung; das Problem der Integrität angesichts der Realität von Gewalt: der Kampf um Anerkennung der eigenen Menschenwürde, um Selbstwert, um Bindung und Liebe in Freiheit; das Problem der Demokratie angesichts der Realität von Dominanz und Herrschaft: der Kampf um Teilhabe, um die eigene Stimme, um Gewicht und Bedeutung im gesellschaftlichen Ganzen.

Mit der kritischen Dekonstruktion klassischer Konzepte von Subjekt und Autonomie sind viele Selbstverständlichkeiten herkömmlicher Politik infrage zu stellen. Das Bemühen um lebbares Leben, um Geschlechterdemokratie, um eine neue Kultur der Geschlechter, braucht trotz alledem Handlungsorientierungen und konkrete Ansatzpunkte für eine konkret auszugestaltende Praxis. Geschlecht kann daher nicht nur als diskursives Konstrukt aufgefasst, sondern muss immer auch als sich sehr konkret darstellender Erfahrungszusammenhang gesehen werden. Die Rede von doing gender verweist letztlich auf beide Dimensionen - auf gesellschaftlich-kulturelle Zuschreibungsprozesse, auf individuelle wie kollektive Identitätsbildungsprozesse, wie auch auf Alltagserfahrung und Handlungspraxis. Vor diesem Hintergrund kann „Geschlecht“ als politische Kategorie gefasst werden, mit der Problemlagen thematisiert und auf die bezogen auch konkrete Konzepte entwickelt werden können.

Im Laufe der bisherigen Darlegung wurde deutlich: Frauen- und Geschlechterforschung konnte und kann nicht einfach als Kontrapunkt gegen das etablierte System der Wissenschaft entworfen werden; vielmehr ist viel allgemei- 
ner nach ihren Machtwirkungen zu fragen - und wie diese Machtwirkungen kritisch reflektiert und gestaltet werden können. Ich möchte abschließend einen Denkvorschlag ins Spiel bringen, der dabei - auch und gerade im Kontext eines feministischen Selbstverständnisses - vielleicht zur Orientierung dienen kann. Er bezieht sich auf die - inzwischen mehrfach angeklungene - grundlegende Frage, wie Frauen- und Geschlechterforschung - auch in ihrer Veränderung, Differenzierung und Komplexität - wahrgenommen und kritisch gewürdigt werden kann, wie die damit verbundenen ,gesellschaftlichen Erfahrungen“" in all ihrer Brüchigkeit und Ambivalenz rekonstruiert, (selbst)kritisch reflektiert und gesellschaftlich sinnvoll weiterbearbeitet werden können. Hierzu braucht es meines Erachtens ein „Gedächtnis der Konflikte“ sowie ein „Denken des Transformativen“, das sich auch mit einem „Denken der Menschen in Kräftefeldern“ verbindet. Der Denkvorschlag zielt darauf, Frauenund Geschlechterforschung als „Gedächtnisort“ oder Gedächtnisspeicher zu fassen.

Mit einem „Denken der Menschen in Kräftefeldern“ können bspw. auch diejenigen ,Praxen der Kritik‘ ins Feld der Wahrnehmung gelangen, die nicht unbedingt den herkömmlichen Bildern von Opposition entsprechen: Praxen, die sozusagen direkt gegen das vorgehen, was einer nicht passt, was eine überhaupt erst ,regierbar' macht (die Zuschreibung von subjektiven Eigenschaften, die naturalisierende Festlegung, das Einordnen und im Raum Verteilen, das Hierarchisieren). Ein solches Denken muss sich nicht auf ,Identität/en“ und ,Zugehörigkeiten' berufen, sondern kann die widerspenstigen Kräfte in den Individuen, Gruppen und ,Szenen' selbst aufsuchen, die in Selbstverständnissen, Positionen und anderen Definitionen nicht eindeutig aufgehen (vgl. hierzu insgesamt Wenta 1997; Maurer 2001).

In der Frauen- und Geschlechterforschung geht es letztlich um ganz konkrete Praktiken, um die auch selbstreflexive Erforschung, Analyse (und Gestaltung) alltäglicher Machtmechanismen, um damit für das ,Einhaken von Herrschaftsformen' zu sensibilisieren, jene vielleicht sogar zu verändern. Es entsteht die gestalterische Frage, wie Machttechniken und -strukturen anders geformt werden können. Für die AkteurInnen der Frauen- und Geschlechterforschung könnte das heißen, am jeweiligen Ort, an dem eine/r Einfluss hat, mit den verschiedensten Techniken gegen die Mikromechanismen der Macht anzugehen - mit Strategien der Beweglichkeit, der Vielfältigkeit, und von wechselnden Positionen aus.

Der Historiker Philipp Sarasin (2003) schlägt vor, ein „Subjekt der agency“ zu denken, um ,jenseits der Fallen der Bewusstseinsphilosophie“ fragen zu können, ,wie Subjekte sich in den Widersprüchen der symbolischen Ordnung als eigenständige, eigensinnige Produkte dieser Ordnung einnisten“" und 
verweist dabei auch auf die hier an anderer Stelle bereits erwähnte Gouvernementalitäts-Debatte im Anschluss an Michel Foucault, die sich ebenfalls auf der Spur der Möglichkeiten bewegt, ,die taktischen Dispositive der Macht umzukehren und in Instrumente des Widerstandes zu verwandeln“" (Sarasin 2003, S. 55).

Die theoretischen Überlegungen von Sarasin werden hier nicht zuletzt deshalb angeführt, weil er sich in seinen Texten - als Geschichtstheoretiker resp. als geschichtstheoretisch Reflektierender - mit einem Spannungsfeld auseinandersetzt, das auch für die hier vorgestellten Überlegungen von zentraler Bedeutung ist: dem Spannungsfeld von Diskurs, Politik und Erfahrung. In diesem Spannungsfeld erscheint ein sozial situiertes, kon-textuiertes „Wissen“ (im Sinne von „Erfahrung“ und „Erkenntnis“) angebracht, das seiner Gespaltenheit, seiner Vieldeutigkeit und Instabilität immer wieder gewahr wird.

„Das neue Bewusstsein, das sich in der Bewegung ausdrückt und vermittelt, setzt sich zusammen aus tausend Facetten, in denen sich die Erfahrungen der beteiligten Frauen spiegeln. Auf eine Bewegung, die sich durch Praxis, Diskussionen und erste Verallgemeinerungsversuche entfaltet, passt kein geschlossenes Theoriekonzept; es würde den Reichtum der Ansätze in vorgestanzte Begriffe einschließen, dem Widerstand den Atem nehmen." So schrieb Michaela Wunderle in ihrem Buch „Politik der Subjektivität“ Ende der 1970er Jahre. Vielleicht gilt diese Beschreibung ja auch für die Frauenund Geschlechterforschung heute?

Meines Erachtens kann ein Bewusstsein von der Geschichte feministischer Denk-Bewegungen (die zur Ausbildung des Feldes „Frauen- und Geschlechterforschung" ja erst geführt haben) ein Bewusstsein für so etwas wie eine „Geschichte der Problematisierungen“ im Sinne Michel Foucaults ermöglichen. Mit dem Wissen über „die Kämpfe der Vergangenheit“ entsteht eine andere Wahrnehmung der Konflikte der Gegenwart - die eigenen Erfahrungen können, mit zeitlicher Tiefe versehen, auf einer anderen Ebene reflektiert werden.

Die Arbeit gälte also der Kultivierung eines ,gesellschaftlichen Gedächtnisses" - auch im Hinblick auf die Frauen- und Geschlechterforschung in der Erziehungswissenschaft. Gemeint ist ein Gedächtnis der damit historisch verbundenen Auseinandersetzungen (um Bedeutung, um Ressourcen, um Zugang und Zugehörigkeit). Um das zu erreichen, braucht es eine differenzierte und kritische Bilanz dessen, was tatsächlich erreicht worden ist - und um welchen Preis. Die Erfahrungen damit sind „gespeichert“ - z.B. in einem Netzwerk, in einer „Organisation“ oder „Struktur“ wie der Sektion „Frauen- und Geschlechterforschung in der DGfE", die in ihrer langjährigen Geschichte institutionell nun weit über die ersten überschaubaren Anfänge als „,AG“ hinaus 
„etabliert“ ist. Gespeichert in der hier praktizierten und - auch in der Kontroverse! - kultivierten Art der Problemwahrnehmung und -deutung, gespeichert in Verfahren und - mehr oder weniger - geteilten (theoretischen) Verständigungshorizonten, im - wenn auch unterschiedlich benutzten - theoretischen wie methodologischen Instrumentarium. Wird diese Funktion der Frauen- und Geschlechterforschung als „Gedächtnisort“, als „Erfahrungsspeicher“ ernst genommen, dann lässt sich - so die damit verbundene Hoffnung - Entwicklungen entgegenwirken, die drohen das kritische Potential der eigenen Arbeit zu neutralisieren.

\section{Literatur}

Adkins, Lisa/Lury, Celia (1999): The labour of identity: performing identities, performing economies. In: Economy and Society, no. 4 (vol. 28)

Adkins, Lisa (2004): Gender and the post-structural social. In: Barbara L. Marshall/ Anne Witz (Ed.): Engendering the social. Feminist encounters with sociological theory. Mc Gruw Hill, Open University Press

Bauer, Silvia (1999): „Beyond“ the Gender Principle. Post-Gender Theories and Contemporary Culture. In: Kati Röttger/Heike Paul (Hg.): Differenzen in der Geschlechterdifferenz - Differences within Gender Studies. Aktuelle Perspektiven der Geschlechterforschung, Berlin

Bitzan, Maria (1996): Geschlechterhierarchie als kollektiver Realitätsverlust - zum Verhältnis von Alltagstheorie und Feminismus, in: Klaus Grunwald et al. (Hg.): Alltag, Nichtalltägliches und die Lebenswelt. Beiträge zur lebensweltorientierten Sozialpädagogik, München

Bitzan, Maria/Funk, Heide/Stauber, Barbara (1998): Den Wechsel im Blick. Methodologische Ansichten feministischer Sozialforschung. Hrsg. vom Tübinger Institut für frauenpolitische Sozialforschung e.V., Pfaffenweiler

Bröckling, Ulrich et al. (Hg.) (2000): Gouvernementalität der Gegenwart. Studien zur Ökonomisierung des Sozialen, Frankfurt a.M.

Bührmann, Andrea (1995): Das authentische Geschlecht. Die Sexualitätsdebatte der Neuen Frauenbewegung und die Foucaultsche Machtanalyse, Münster

Bührmann, Andrea (1998): Die Normalisierung der Geschlechter in Geschlechterdispositiven, in: Hannelore Bublitz (Hg.): Das Geschlecht der Moderne. Genealogie und Archäologie der Geschlechterdifferenz, Frankfurt a.M.

Ernst, Waltraud (1999): Diskurspiratinnen, Wien

Friebertshäuser, Barbara (1997): Geschlechtertrennung als Innovation. Etappen geschlechtsbezogener Jugendarbeit im 20. Jahrhundert. In: Barbara Friebertshäuser et al. (Hg.): Sozialpädagogik im Blick der Frauenforschung, Weinheim 
Foucault, Michel (1993): About the Beginning of the Hermeneutics of the Self. Two Lectures at Dartmouth. In: Political Theory, no. 2 (vol. 21), London/New Delhi

Foucault, Michel (1996): Der Mensch ist ein Erfahrungstier. Gespräch mit Ducio Trombadori, Frankfurt a.M.

Foucault, Michel (1999): In Verteidigung der Gesellschaft. Vorlesungen am Collège der France (1975-76), Frankfurt a.M.

Geissler, Birgit / Oechsle, Mechthild (1996): Lebensplanung junger Frauen. Zur widersprüchlichen Modernisierung weiblicher Lebensläufe, Weinheim

Goffman, Erving (1994): Interaktion und Geschlecht, Frankfurt a.M.

Hagemann-White, Carol (1998): Identität - Beruf - Geschlecht. In: Mechthild Oechsle/Birgit Geissler (Hg.): Die ungleiche Gleichheit. Junge Frauen und der Wandel im Geschlechterverhältnis, Opladen

Hassauer, Friederike (1994): Homo. Academica. Geschlechterkontrakte, Institution und die Verteilung des Wissens, Wien (Passagen Heft 10)

Kahlert, Heike (1996): Weibliche Subjektivität, Frankfurt a.M.

Kehlenbeck, Corinna (2001): „,.. und was heißt das für die Praxis?“ Historische Entwicklungslinien der Frauen- und Geschlechterforschung im Spannungsverhältnis zwischen Theorie und Praxis - aufgezeigt an der sozialpädagogischen Diskussion über eine geschlechterdifferenzierende Jugendhilfe. In: Bettina Fritzsche et al. (Hg.): Dekonstruktive Pädagogik. Erziehungswissenschaftliche Debatten unter poststrukturalistischen Perspektiven, Opladen

Kurz-Adam, Maria (1994): Spaziergang durch den Feminismus? Versuch einer Standortbestimmung der Frauenforschung zwischen modernen Weltbildern und postmoderner Vielfalt. In: Gerlinde Seidenspinner (Hg.): Frau sein in Deutschland. Aktuelle Themen, Perspektiven und Ziele feministischer Sozialforschung, Weinheim

List, Elisabeth (1996): Politik, Geschlecht und Lebensform. Perspektiven feministischer Theorie und Praxis. In: Ita Maria Grosz-Ganzoni (Hg.): Widerspenstige Wechselwirkungen. Feministische Perspektiven in Psychoanalyse, Philosophie, Literaturwissenschaft und Gesellschaftskritik, Tübingen

Lorber, Judith (1999): Gender-Paradoxien, Opladen

Maurer, Susanne (1996): Zwischen Zuschreibung und Selbstgestaltung. Feministische Identitätspolitiken im Kräftefeld von Kritik, Norm und Utopie, Tübingen

Maurer, Susanne (2001): Das Soziale und die Differenz. Zur (De-)Thematisierung von Differenz in der Sozialpädagogik. In: Helma Lutz/Norbert Wenning (Hg.): Unterschiedlich verschieden. Differenz in der Erziehungswissenschaft, Opladen

Maurer, Susanne (2004): Zum Verhältnis von Frauenbewegungen und Sozialer Arbeit um 1900. Versuch einer historisch-systematischen (Re-)Kontextualisierung nebst Überlegungen zu einer reflexiven Historiographie in der Sozialpädagogik (Habilitationsschrift), Hildesheim

Metz-Göckel, Sigrid (1993): „Permanenter Vorgriff auf die Gleichheit“. Frauenforschung in Westdeutschland. In: Gisela Helwig/Hildegard Maria Nickel (Hg.): Frauen in Deutschland 1945-1992, Berlin 
Metz-Göckel, Sigrid (1998): Mikropolitik in den Geschlechterbeziehungen: Selbstvertrauen, Anerkennung und Entwertung. In: Mechthild Oechsle/Birgit Geissler (Hg.): Die ungleiche Gleichheit. Junge Frauen und der Wandel im Geschlechterverhältnis, Opladen

Modelmog, Ilse/Gräßel, Ulrike (Hg.) (1994): Konkurrenz \& Kooperation. Frauen im Zwiespalt? (Beiträge zur Jahrestagung der Sektion Frauenforschung in der Deutschen Gesellschaft für Soziologie), Hamburg

Muraro, Luisa (1993): Die symbolische Ordnung der Mutter, Frankfurt am Main/ New York

Nadig, Maya/Erdheim, Mario (1984): Die Zerstörung der wissenschaftlichen Erfahrung durch das akademische Milieu. Ethnopsychoanalytische Überlegungen zur Aggressivität in der Wissenschaft. In: Psychosozial, 7. Jahrgang, Heft 12

Pühl, Katharina/Schulz, Susanne (2001): Gouvernementalität und Geschlecht - Über das Paradox der Festschreibung und Flexibilisierung der Geschlechterverhältnisse, Königsstein/Ts.

Rauschenbach, Brigitte (1995): Erkenntnispolitik als Feminismus. Denkformen und Politikformen im feministischen Bildungsprozess der Erfahrung, in: Die Philosophin, Heft 11/1995

Sarasin, Philipp (2003): Geschichtswissenschaft und Diskursanalyse, Frankfurt a.M.

Schmidt, Andrea (2001): Poststrukturalistische Perspektiven. Ein Beitrag zur Reflexion sozialpädagogischer Handlungspraxen. In: Bettina Fritzsche et al. (Hg.): Dekonstruktive Pädagogik. Erziehungswissenschaftliche Debatten unter poststrukturalistischen Perspektiven, Opladen

Schulze, Kristina (2002): Der lange Atem der Provokation. Die Frauenbewegung in der Bundesrepublik und in Frankreich 1968-1976, Frankfurt a.M.

Soiland, Tove (2004): Kritische Anmerkungen zum Machtbegriff in der GenderTheorie auf dem Hintergrund von Michel Foucaults Gouvernementalitätsanalyse (noch unveröffentlichtes Manuskript, erscheint im nächsten Heft der Zeitschrift Widersprüche, 2005)

Wenta, Anke (1997): Individualität serienmäßig. Zur Produktivität der Kategorie Macht für die Praxis der Sozialpädagogik am Beispiel des Rassismus, unveröffentlichte Diplomarbeit, Tübingen 



\section{„Doing teacher" - professionelle Inszenierungen in Wechselwirkung von institutionellen Arrangements und sozialer Praxis}

\section{Sabina Larcher}

Pädagogisches Handeln zeichnet sich - bedingt durch die bestehenden Strukturen des professionellen Feldes und Kontextes - durch Individualität, Reflexivität, Isolation, Autonomie, hohe personale Ressourcen und Ungewissheit aus. Lehrer/innen sind deshalb unter hoher Ungewissheit Handlungsspannungen zwischen Diagnose, Schlussfolgerung und Anwendung ausgesetzt. Ungewissheit wird letztlich situativ handelnd bearbeitet, nicht aufgelöst. Professionalität im Lehrberuf wird nun denjenigen zugestanden, die in der Lage sind, solche Friktionen zu ertragen, produktiv zu balancieren und immer wieder neu die Voraussetzungen und Implikationen solchen Handelns in Ungewissheit zu reflektieren und dafür die Verantwortung auf der Basis professioneller Zuständigkeit zu übernehmen (Rabe-Kleberg 1996, S. 295). Darin aufgehoben ist eine Topografie des Arbeitsraumes, der sich in der Analyse durch anomische Strukturen, Unübersichtlichkeit, hohe Komplexität, geringe Standardisierung und stetige Belastung charakterisieren lässt. Die präsentierte Topografie und das erstellte Anforderungsprofil an professionell Handelnde verweist auf gesteigerte Figurationen wie der der „idealen Lehrperson“, der „,des guten Lehrers“ - oder aktueller - der des ,professionellen Lehrers“ oder des „Experten“. Diesen eingeschrieben sind Geschlechterkonstruktionen und arrangements die auf Dualismen wie „Privat - Öffentlich“, „Erziehung - Bildung“ oder „Fürsorge - Gerechtigkeit“" und deren jeweiligen Deutungsebenen beruhen. Selten werden diese so explizit benannt und prominent präsentiert wie das etwa in der Formulierung Konzipierung eines ,weiblichen Selbstkonzepts des Lehrers“ durch Lenzen (2003) nach zu lesen ist. Denn im Zuge bürokratischer und ökonomischer Semantiken (Gonon 2003), wird die Tätigkeit und das Handeln von Lehrer/innen zunehmend mit SelbstmanagementTermini beschrieben und rationalisiert. Dadurch werden die Handelnden mit Zumutungen konfrontiert, die auf ein Selbst- und Weltverhältnis im beruflichen Handlungskontext verweisen, dessen Konstituierung durch das „Subjekt" selbst in der Reflexion und der Begründung der unterschiedlichsten Bedingungsfaktoren geleistet werden muss. Grenzziehungsprozesse im Berufs- 
feld, die aufgrund von Strategien und Verfahren sozialer Schließung entstehen und den Handlungsspielraum der Einzelnen entscheidend mitprägen, geraten so aus dem Blickfeld.

Lehrerinnen und Lehrer sind im angesprochenen diffusen, fluiden und inhomogenen Kontext nicht nur Adressat/innen von Konstituierungspraxen, sondern auch deren Träger/innen. Unterschlagen wird dabei - speziell in der Thematisierung des Berufseinstiegs von Lehrpersonen -, dass in der Regel von „geschlechtsneutralen“ beruflichen Kontexten und entsprechenden Professionalitätskonzepten ausgegangen wird, denen Einheits- und Homogenitätsvorstellungen sowie ein kontinuierlicher Zuwachs von Expertise zu Grunde liegen. Mögliche Qualitäten wie Ungleichzeitigkeit, Brüchigkeit oder Fragilität werden dabei ebenso wenig zulassen, wie das Hinterfragen von Berufskonstruktionen und Codierungen des Arbeitsfeldes. Damit ist auch die systematische Bearbeitung von Prozessen im Berufsfeld hinsichtlich einer „Gender Factory“ (Fenstermaker 1985) kaum möglich. Damit sind Mitgliedschaftsentwürfe (Schimank 2000) nicht transparent und Inszenierungen, Identifikationen oder Abgrenzungen für Professionelle erschwert. Fragen nach dem Verbleiben im oder dem Aussteigen aus dem beruflichen Feld müssten unter dieser Perspektive systematisch betrachtet werden.

Der vorliegende Beitrag beleuchtet ausgehend von der Annahme, dass Professionalität als Produkt sozialer Praxis, institutioneller Arrangements und kultureller Deutungen zu konzipieren sei, die Frage, wie die Bedeutungen von „Lehrerin“ oder „Lehrer“ durch Berufsanfänger/innen auf der Mikroebene des Schulalltags bearbeitet werden. Diese Bedeutungen sind diskursiver Bestandteil eines sozialen Signifikationssystems, die auf Geschlechterarrangements auf der Mesoebene der Berufskonstruktion verweisen und damit in engem $\mathrm{Zu}-$ sammenhang mit der Berufsstruktur - die mit dem Begriff „,feminisiert“ gefasst wird - wie auch dem beruflichen Feld stehen. In Anlehnung an Wetterer (1998; 2002, S. 39ff.) wird anhand der Re-Lektüre eigener Forschungsdaten exemplarisch dargestellt, wie sich in diesem Bereich Historisierung und Kontextualisierung des „doing gender“ und des „doing work“ aufeinander beziehen lassen, was an dieser Stelle arbeitshypothetisch und im Kontext des Berufseinstiegs tentativ mit einem konzeptionellen „doing teacher“ umschrieben wird.

In einem ersten Schritt werden im Folgenden grundlegende Befunde aus dem eigenen Forschungsprojekt berichtet, die den Kontext für eine skizzenhafte Betrachtung des angesprochenen Problemfeldes bilden. Dies soll mit zwei Perspektiven geschehen: Zum einen werden institutionelle Arrangements) skizziert und zum anderen exemplarisch die Mikroebene (soziale Praxis) einbezogen. 


\section{„Jetzt würde ich mal sagen, ich habe mein erstes Jahr mal gemacht und es ist vieles schief gelaufen, und ich habe extrem viel gelernt. ${ }^{\text {" } 1}$}

Ausgangspunkt des Beitrages bilden Daten und Ergebnisse aus einem im Jahr 2002 abgeschlossenen Forschungsprojekt zum ersten Berufsjahr von Lehrerinnen und Lehrern an Volksschulklassen in der Deutschschweiz. ${ }^{2}$ Einem mehrperspektivischen Untersuchungssetting ${ }^{3}$ verpflichtet, konnte die Studie aufzeigen, dass aufgrund des sehr individuellen Verlaufs des Berufseinstiegs, das Postulieren eines normativen Verlaufs wie er in der Literatur zu diesem Thema dargestellt wird, zumindest fragwürdig ist. Die Ergebnisse legten nahe, dass die Entwicklung von Kompetenz im beruflichen Feld nicht in Stadien oder Phasen zu denken ist, sondern als dynamischer Prozess, der immer wieder und in neuen Bezügen ähnliche Probleme definiert und zum Inhalt hat. Die Beschäftigung mit der Entwicklung von Professionalität machte zudem deutlich, dass sich junge Lehrpersonen bereits in einem laufenden Prozess befinden, und mit dem Eintritt in die Praxis berufliche Selbstentwürfe zur Disposition stellen. Es findet in diesem Sinne kein Anfang statt, sondern eine

Originalzitat aus einem der geführten Interviews: Proband 3, 3. Interview.

2 Larcher, Sabina (2005). Einstieg in den Lehrberuf. Untersuchungen zur Identitätsentwicklung von Lehrerinnen und Lehrer im ersten Berufsjahr. Bern: Haupt-Verlag (in Druck).

3 Es wird von einer quantitativen und qualitativen Längsschnittuntersuchung, die während des ersten Praxisjahres von jungen Lehrer/innen durchgeführt wurde, berichtet. Ziel der Untersuchung war es, einen qualitativ sowie quantitativ abgesicherten Einblick in die Berufseinmündungsphase hinsichtlich einer Entwicklung beruflicher Identität zu erhalten. Die Grundlage der Untersuchung bildet ein Konzept von Frey und Hausser (1987) zur Erfassung und Beobachtung von Identitätsregulationen, das erlaubt, den dynamischen Prozess abzubilden. Dieses Konzept ermöglicht es, den Fokus auf die junge Lehrperson und deren Entwicklung in der Wechselwirkung mit ihrer Umwelt zu richten. Daraus ergaben sich eine Innen- wie Außenperspektive der Betrachtungen, wobei die Innenperspektive den Ausgangspunkt der Untersuchung darstellt. Von diesem Punkt aus wurde gefragt, welche Ereignisse und Anteile der Außenperspektive (berufliches Umfeld) relevant und bedeutsam für die Entwicklung der Innenperspektive (berufliche Identität) sind. Durch mehrere Erhebungen (Längsschnitt) sollte der beginnende oder schon laufende Prozess der beruflichen Identitätsentwicklung erfasst werden, wobei die Subjektivität der Proband/innen einen wichtigen Stellenwert einnahm. Insgesamt wurden drei Erhebungen sowohl mit qualitativen wie quantitativen Instrumenten geplant und durchgeführt (vor dem Praxiseinstieg, nach dem ersten Praxissemester und gegen Ende des ersten Praxisjahres der jungen Lehrer/innen). Die Stichprobe umfasste eine Teilgruppe der Abgänger/innen eines Schweizerischen Lehrer/innenseminars, welche in zwei Subgruppen (quantitativ, $n=40$; qualitativ $n$ $=10$ ) aufgeteilt wurde, die mit je unterschiedlichen Instrumenten befragt wurden. Die Proband/innen waren zum Zeitpunkt der ersten Erhebung zwischen 20 und 23 Jahre alt. Die prozentuale Verteilung der Frauen und Männer betrug $82 \%$ bzw. 18\%, was in etwa der von Lehrerinnen und Lehrern an der Volksschule entspricht (hochgerechnet auf 100\%-Stellen). 
Weiterführung bereits vorhandener beruflicher Selbstbilder unter veränderten Vorzeichen.

Die Ergebnisse zeigten, dass der Berufseinstieg von den Befragten ausgesprochen ambivalent erlebt wurde, d.h. als eine Phase mit starken Belastungen, bei gleichzeitiger Zufriedenheit über die erreichten Erfolge und positiven Erfahrungen in der Zusammenarbeit mit den Kindern und Jugendlichen in den eigenen Klassen. Obwohl sich innerhalb der Stichprobe große individuelle Unterschiede in der Gestaltung und dem Erleben des ersten Praxisjahres ergaben, konnten übereinstimmende Komponenten und Merkmale sowie Faktoren des Berufseinstiegs herausgefiltert werden, die die wahrgenommenen Anforderungen aus dem pädagogischen Arbeitsfeld und deren aktive Bearbeitung beleuchten: Allgemein war die Wahrnehmung der Probandinnen bestimmt durch das auch in der Forschungsliteratur vermittelte Bild der Vielfalt der Tätigkeit, der Komplexität der Praxis, der potenziellen Offenheit und Vieldeutigkeit von beruflichen Situationen, der begrenzten Durchschaubarkeit der Bedingungen des pädagogischen Handelns. Dies machte so in ihrem Erleben korrespondierende Fähigkeiten des Umgangs mit ständig wechselnden Anforderungen erforderlich. Diese situativ rasch wechselnden und - in der Perspektive der Proband/innen - noch zu entwickelnden oder zu verstärkenden Fähigkeiten und Handlungsmuster verwiesen auf unterschiedlichste Aspekte kompetenten pädagogischen Handelns. Die Berufsanfänger/innen versuchten deshalb gezielt, das eigene berufliche Selbstbild und ihr pädagogisches Konzept mit den aus verschiedenen Bezugssystemen wahrgenommenen Anforderungen in Einklang zu bringen, sowie differente Aspekte der Selbstwahrnehmung stimmig zu strukturieren. Sie orientierten sich dabei an ihren Schüler/innen, an deren Eltern, an Professionsentwürfen und an individuell gewählten Schlüsselpersonen, was teilweise zu Irritationen und Überforderungen führte. Diese Bezugsgrößen ermöglichten ihnen jedoch zumindest eine Orientierung im laufenden Konstruktionsprozess. Das Fehlen solcher Feedbacks provozierte wiederum seitens der Berufsanfänger/innen große Unsicherheiten. Sie hatten den Eindruck, sich in einem ,diffusen beruflichen Feld oder Raum“ zu bewegen. Aufgrund der starken Dynamik hinsichtlich des beruflichen Selbstkonzeptes ließ sich weiter schließen, dass sich für die jungen Lehrpersonen eine Anzahl von Momenten und Situationen der Selbstbehauptung ergeben und umgekehrt spezifisch substantielle Bedrohungen bezüglich der dargestellten beruflichen Selbstbilder vorgelegen hatten.

Allgemein konnte in der Untersuchungsgruppe eine äußerst aktive und produktive Realitätsverarbeitung beobachtet werden, die auf Balanceaufgaben bezüglich Selbstregulation, Anpassung und Selbstverwirklichung, interpretiert als Balancebemühungen, in verschiedenen Bereichen aufmerksam machte. 
Der Übergang von der Ausbildung in den Lehrberuf konnte davon ausgehend für alle Befragten als Krisenlage beschrieben werden, auch wenn sich die Berufsanfänger/innen ihrer Tätigkeit gewachsen fühlten, ihr Handeln als stimmig und kompetent erlebten und mit ihrem ersten Praxisjahr zufrieden waren. Das Beenden der Ausbildung und die Aufnahme der Berufstätigkeit stellte einen Einschnitt in der Biografie dar, als ,getting into the adult world“ (Oja 1989).

Die Studie thematisierte in der Ausgangsfrage ein Problemfeld, das in der Tradition der Erforschung von Wirkungen der strukturellen und institutionellen Bedingungen des Lehrberufs auf die Lehrenden liegt. Das genuine Interesse richtete sich davon ausgehend auf die Problematisierung und kritische Diskussion normativer Konzepte hinsichtlich der Entwicklung beruflicher Kompetenzen und arbeitete deshalb in der Analyse des Materials nicht mit der Kategorie Geschlecht. Zudem sollte vermieden werden, dass das ,(...) Vorhandensein körperlich weiblicher Wesen als Indiz dafür (gilt), dass ein Differenzsystem, das uns bereits vertraut ist, seine Wirkung entfaltet" (Collier/Yanagisako 1987, zit. nach Scott 1998, S. 35f.. In der Bearbeitung des Datenmaterials zeigte sich jedoch, dass die Lehrerinnen und Lehrer in der Auseinandersetzung mit ihren beruflichen Selbstbildern wiederholt auf die eingangs erwähnten verdeckten Grundstrukturen der Mitgliedschaftsentwürfe und Grenzziehungen verwiesen, die im Folgenden in der Skizzierung eines Bedeutungshorizontes in aller Kürze historisiert und kontextualisiert werden sollen.

\section{Diachrone Perspektive: Was ist Erziehung? - Ein Geschäft!}

Als Josephine Stadlin 1845 in der ersten Nummer der Zeitschrift „Die Erzieherin. Eine Zeitschrift über weibliche Erziehung, dem Verein schweizerischer Erzieherinnen gewidmet von einigen Mitgliedern dieses Kreises. ${ }^{\text {"4 }}$ die Frage stellte: „Was ist Erziehung?“, und den Leserinnen und Lesern im Untertitel des Artikels die Antwort gab: „Ein Geschäft", thematisierte sie mit der Anschlussfrage „Wem soll dieses Geschäft übertragen werden?“ ein Diskussionsfeld, das bis heute Bestand und im zeitlichen Verlauf zahlreiche Umdeutungen wie auch moralische Aufladungen erfahren hat.

Die Debatten um die Beteiligung von Frauen am öffentlichen Unterricht der Volksschule und zur Lehrer/innenfrage im Allgemeinen fanden in den schweizerischen Kantonen teilweise bis in die 1920er-Jahre statt. Das föderalistische Sys-

4 Sie gab die Zeitschrift „Die Erzieherin, Eine Zeitschrift über weibliche Erziehung, dem Verein schweizerischer Erzieherinnen gewidmet" im Druck und Verlag von Friedrich Schulthess in Zürich heraus. 
tem ermöglichte sehr heterogene Ausbildungsmodi und -modelle für Lehrerinnen und für Lehrer. Wer befugt war an öffentlichen Schulen zu unterrichten, d.h., das Geschäft auszuüben und damit eine Ausbildung für die Lehrtätigkeit an der Volksschule an den kantonalen Lehrer/innenseminaren zu erhalten, wurde gesamtschweizerisch laut Bundesverfassung den einzelnen Kantonen zur Reglementierung überlassen. Der allgemeine Zugang für Frauen zu den kantonalen Ausbildungsstätten wurde dadurch erschwert oder gar verunmöglicht. Bemühungen um eine Professionalisierung des Lehrberufs, die etwa in Forderung nach oder in der Durchsetzung von veränderten, d.h., verlängerten, akademisierten oder verstärkt berufspraktischen Ausbildungsmodi sichtbar wurden, riefen zugleich Befürchtungen hinsichtlich einer Abwertung des Berufsstandes hervor, falls diese Maßnahmen auch die Ausbildung von Lehrerinnen betreffen sollten. Die Diskurse um die außerhäusliche Erwerbstätigkeit der Frau im öffentlichen Bildungssystem war im Wesentlichen durch Deutungsmuster oder Diskursfiguren wie „natürliche Beruf der Frau“, „,soziale Not unverheirateter höherer Töchter", ,Jungfrauenberuf“, „Naturberuf", „Bildung schadet dem Frauenkörper“, „Frauenkörper und Volkskörper“, „Mutter und Weltweib“ gekennzeichnet. Diese Naturalisierung vollzog sich sowohl in Debatten um rechtliche Gleichheit zum einen wie auch um die Erwerbsarbeit zum andern in Rekurs auf fehlende moralische und rationale Kompetenzen von Frauen, und im Verlaufe des Jahrhunderts verstärkt mit biologistischen Theorien zur natürlichen weiblichen Defizienz (Studer 2000, S. 94).

Die Integration von Frauen in den Lehrberuf kann in aller Verkürzung für den schweizerischen Kontext als „bottom up“-Prozess umschrieben werden, der zwei Phasen umfasst: In einem ersten Schritt vollzog sich der Zugang der Frauen zur Lehrtätigkeit an öffentlichen Schulen in den Kantonen, d.h., Frauen wurden als Lehrerinnen durch die Schulgemeinden angestellt und arbeiteten hauptsächlich an den Unterstufen der Volksschule ${ }^{5}$. Erst in einem zweiten Schritt vollzog sich ihr Zugang zu einer staatlichen und damit „öffentlichen“ Ausbildung, die als äquivalent zur Ausbildung der angehenden Volksschullehrer bezeichnet werden kann. Die Frage der staatlichen Ausbildung von Frauen zu Lehrerinnen und der Beteiligung von Frauen am öffentlichen Unterricht in der zweiten Hälfte des 19. Jahrhunderts wurde jedoch in einer Pha-

5 Der Erwartungshorizont hinsichtlich einer weiblichen Berufskonstruktion zeigt folgendes Zitat exemplarisch: „Als solche fand er (Inspektor; SL) bei ihr (der examinierten Lehrerin; SL): Begeisterung und vollständige Hingabe an ihren Beruf, - selbstlose Liebe zu ihren Kindern (I Cor. 13), - zarte Strenge und ernste Milde in der Disziplin, - immer erneute Vertiefung und methodische Durcharbeitung der verschiedenen Unterrichtsgegenstände, Demut, Geduld, Sanftmut und wahre Frömmigkeit." Anonyme Zusendung eines Schulinspektors, In: Schweizerische Lehrerinnen-Zeitung, 1896, Nr. 6, S. 81-84. 
se thematisiert, als der Lehrberuf sich zu einem Beruf entwickelte, der es zum einen ,intelligenten armen Knaben“ aus der unteren ländlichen Mittelschicht erlaubte sollte sozial aufzusteigen. ${ }^{6}$ Andererseits wurde der Lehrberuf als Möglichkeit gesehen, den „Töchtern“ zu einer angemessenen beruflichen Betätigung zu verhelfen, die dem gängigen bürgerlichen Frauenbild entsprach. Obwohl sich in dieser Frage politische Spannungen ergaben, war den tätigen Lehrerinnen sehr deutlich, dass die Lehrerinnenfrage neben der allgemeinen Schul- und Lehrerpolitik einher ging und diskursiv sowohl an die Frauenfrage wie auch an die soziale Frage gekoppelt war. ${ }^{7}$ Dieser Themenkomplex bestimmte Ausbildungsdebatten und Diskussionen um die außerhäusliche Tätigkeit von Frauen im Allgemeinen.

\section{Synchrone Perspektive: „Sind Lehrerinnen den Jungen nicht gewachsen?" - Beobachtungen zur Feminisierungsdebatte 8}

Es hat sich gezeigt, dass bereits die Integration von Frauen in die Lehrberufe und ihre sukzessiv erfolgte formale Gleichstellung innerhalb des beruflichen Feldes ein Problem formuliert, das die Öffentlichkeit und die Vertreter der

6 Der Thurgauer Lehrer Vollenweider sprach sich beispielsweise 1872 in einem gesamtschweizerisch stark beachteten Vortrag dafür aus, dass die Weiterbildung von armen intelligenten Knaben für den Lehrerberuf eine verlockende Umgestaltung des Seminars mit sich bringen würde (Vollenweider, Die Lehrerbildungsfrage im Thurgau, hg. von der Bezirkskonferenz, Huber, Frauenfeld 1872), da die Söhne des Bürgertums die Kantonsschulen, d.h., die Gymnasien besuchten.

7 Folgende Schließungsstrategien wurden in einzelnen Kantonen bis in die 1970er-Jahre angewandt (Hintergrund: Das Frauenstimmrecht wurde in der Schweiz 1971 eingeführt, der Gleichstellungsartikel 1992 in der Bundesverfassung verankert): Es gibt Schulgemeinden mit Besoldungsskalen nach Dienstjahren. Aus dieser Auflistung wird deutlich, dass Lehrerinnen markant weniger verdienen als Lehrer. Die Einteilung lautet bspw. Lehrerinnen, Unterlehrer, Oberlehrer. Zudem gibt es Gemeinden, in denen es für die Lehrerinnen länger dauert (Dienstjahre), bis sie eine Lohnerhöhung erhalten. In verschiedenen Gemeinden dauert es für die Lehrerinnen auch länger, bis sie das Maximum beziehen können. Wo die Rubrik „Lehrerin“ nicht geführt wird, sind die Lehrkräfte an den Unterklassen schlechter bezahlt, was meistens wiederum Frauen betrifft, die fast zu $100 \%$ nur an Klassen der Unterschule eingestellt wurden (Besoldungs- und Wohnverhältnisse der thurgauischen Lehrerschaft, Herbst 1916, Hg: Sektion Thurgau des Schweizerischen Lehrervereins, Huber, Frauenfeld). Vgl. dazu auch Renold, Ursula (1999): „Wo das Männliche anfängt, da hört das Weibliche auf", Dissertation, Universität Bern; Larcher, Sabina/Manz, Karin (2002): „Diese Frauen waren ganz anders, als sie in der Öffentlichkeit hingestellt wurden“, in: Zeitschrift für pädagogische Historiographie, 8, S. 83-89.

8 Vgl. dazu die Beilage der NZZ, 16. Januar 2004. 
einzelnen Berufsgruppen seit gut 150 Jahren periodisch beschäftigt. Dazu gehört, dass die quantitative Zunahme von Frauen als professionell Tätige nach wie vor als Entwicklung eines Berufs hin zu einem Frauenberuf verstanden wird. Mit dem Begriff „Feminisierung“ wird ein solcher Prozess gefasst. Damit werden nicht nur quantitativ strukturelle Veränderung beschrieben, sondern auch Qualitäten verbunden. Geht man davon aus, dass Berufe aus der gesellschaftlichen Definition von Problemen entstehen und sich verändern, dann überrascht die implizite Abwertung der Arbeit von Lehrerinnen im krisengeschüttelten Bildungsbereich keinesfalls. Die eingangs angedeutete Reduktion von Komplexität führt zu einer Instrumentalisierung der Kategorie Geschlecht und verleitet dazu, sie ,zu einem Eimer zu erklären, in den Eigenschaften hineingeleert werden können" (Goffmann 1994, S. 113) und Diskussionen in einem gedanklichen Zirkel leer laufen zu lassen, weil so genannte Unterschiede durch Unterschiedlichkeit erklärt werden (Wetterer 2002, S. 31). Das Soziale wie das Politische werden damit zum Verschwinden gebracht und biologistische Argumentationslinien fokussiert. Diese Vereinfachung löst eine Konfusion zwischen sehr unterschiedlichen Ebenen aus, wie etwa der gesellschaftlichen Ordnungsstruktur, der Berufspolitik, der Bildungstheorie und der Schulpraxis, letztendlich zwischen Diagnose und Beschreibung.

Die Strukturveränderung gibt aktuell dahingehend Anlass zur Sorge, dass sich die Schule und der Unterricht - bedingt durch den Überhang an Lehrerinnen - verstärkt durch einen weiblichen Stil und durch weibliche Unterrichtsstrukturen auszeichnen. Diese Argumentationslogik ist historisch kein neues Phänomen. Sie gewinnt jedoch an Brisanz weil sie aktuell an die Frage gekoppelt wird, wer im Schulsystem die wahren Gewinnerinnen und die eigentlichen Verlierer seien. Eine solch holzschnittartige Darstellung verdichtet die Geschlechterpolarität aufs Neue und verdeckt damit zum einen die feiner, ja fast unsichtbar gewordenen Geschlechterdifferenzen und blendet darunter liegende strukturelle Prozesse aus, nimmt sie gleichsam aus dem Blickfeld; zum andern gilt der Gleichstellungsauftrag als abgeschlossen, ja als allzu erfolgreich: Mädchen sind - gemessen an dieser binären Matrix - gleicher. Von Interesse ist nicht mehr der Prozess der Herstellung von Ungleichheit, sondern das „fertige“ Ergebnis. Damit wird es zunehmend schwieriger die Auseinandersetzungen zwischen sozialen Gruppen oder kollektiver Akteur/innen um Macht und Einfluss zu thematisieren, wie auch das Entstehen von Berufen und deren Grenzen als sozialen Konstruktionsprozess sichtbar zu machen und diese zu benennen (vgl. dazu Wetterer 2002). Nach wie vor scheinen Lehrpersonen weiblichen Geschlechts - oder im angedeuteten bildungspolitischen Kontext erneut - auf das Stereotyp der Mütterlichkeit und damit an Erziehung 
zurück gebunden zu werden. In diesem Zusammenhang wird nicht zuletzt dies historisch ebenfalls nicht neu - das bereits in den einleitenden Überlegungen erwähnte „weibliche Selbstkonzept des Lehrers“ bemüht, das sich weniger durch Vermittlung von Bildungsinhalten, denn durch ein Interesse an Erziehungsfragen auszeichne. Interessant an solchen Diskussionen ist der Umstand, dass das Geschlecht der Lehrkraft in den Mittelpunkt der Betrachtungen rückt.

Der Lehrberuf stellt ein berufliches Feld mit Bedeutungshorizonten dar, das Prozesse der Konstitution von Geschlecht ermöglicht und nicht nur durch die Kategorie „Geschlecht“ strukturiert ist. In diese Überlegungen sollten prospektiv die sich abzeichnenden neuen Formen der Arbeitsteilung - Unterricht, Schulsozialarbeit, Medizin, Schulmanagement - und die neuen Felder Frühpädagogik, Ganztagesschule etc. - einbezogen werden. Das Beharren auf der Differenzierung von horizontaler und vertikaler Segregation ist auch in Zusammenhang mit der Proklamation des „lebenslangen Lernens“ bei gleichzeitigem Rückgang des quantitativen und strukturellen Stellenwertes der beruflichen Erstausbildung von zentralem Interesse. Es stellt sich die Frage, welche Konsequenzen diese Entwicklungen für die professionell Handelnden in diesem Feld haben. Frauen arbeiten nach wie vor mehrheitlich in den weniger gut bezahlten und damit auch weniger angesehenen Bereichen des Bildungssystems. Sie sind mehrheitlich als „Lehrerinnen“ und Männer als „Dozierende oder Professoren" tätig. Und nach wie vor wird die zunehmende Teilzeitarbeit als eine Folge des immer höher werdenden Frauenanteils gesehen und problematisiert, denn konkret gesucht werden in der Praxis Fachleute, die möglichst 100 Prozent in ihrem Beruf arbeiten. Das Aufrechterhalten eines solchen Berufsprofils muss zur Debatte stehen und einen wesentlichen Teil in der Diskussion um die Entwicklung des Lehrberufes bilden.

\section{Figurationen}

Die Skizzen in diachroner wie auch synchroner Perspektive zu Aspekten des institutionellen Kontextes und seinen Arrangements, sollten auf die Dynamik des Berufskonstruktionsprozesses aufmerksam machen. Mit Giddens (1992, S. 77) gehe ich nun davon aus, dass die „Konstitution von Handelnden und Strukturen eine Dualität darstellt" und keine voneinander unabhängigen Phänomene. Struktur ist in Form der Erinnerung und in sozialen Praktiken verwirklicht (ebd.). Auf der Mikroebene, im beruflichen Alltagshandeln, geht es deshalb immer auch darum, den Anforderungen der geschlechtlich „richtigen“ 
und eindeutigen Identifizierbarkeit Rechnung zu tragen: die Darstellung beruflicher Kompetenzen wie auch die Darstellung der Geschlechtszugehörigkeit kann gleichzeitig gefordert oder nötig werden (Wetterer 2002, S. 163). Auch wenn Spielräume im Handeln und der Interpretation der Bedeutungen grundsätzlich bestehen, kann die Verweigerung des Zeigens Irritationen oder weitergehend Sanktionen nach sich ziehen, Probleme und Schwierigkeiten im beruflichen Kontext können die Folge davon sein. Gerade Berufsanfänger/innen müssen sich mit den ,erwirtschafteten Lösungen“ innerhalb eines beruflichen Feldes (Dewe/Radtke 1993) auseinandersetzen.

In der Auseinandersetzung mit dem Berufseinstieg von Lehrerinnen und Lehrern ergab sich im Kontext der bereits angesprochenen Studie ein überraschendes Moment: Im Gegensatz zu den befragten Lehrerinnen äußerten alle männlichen Probanden ${ }^{9}$ große Mühen mit der Klassenführung und beklagten sich über starke Disziplinprobleme. Dies war umso überraschender, da sie über breite und mehrjährige Erfahrungen in der außerschulischen Kinder- und Jugendarbeit verfügten. Die Analysen der Interviews zeigten auf, dass sie sich sehr engagiert mit Leitungsaufgaben und -funktionen auseinandersetzten, diese mit negativ Bedeutungen verbanden und sie mehrheitlich - in Rekurs auf ihr professionelles Konzept, das sie zentral mit Aufgaben des Begleitens und des Coachens verbanden - von sich wiesen. Im Folgenden soll dies schrittweise und exemplarisch aufgezeigt und zur Disposition gestellt werden.

\section{Professionelles Konzept und soziale Praktiken}

In der Analyse der wahrgenommenen Umwelt hat sich für die Gesamtheit der Befragten ein professionelles Konzept herausarbeiten lassen, das in verschiedenen Interaktionsräumen der Lehrer/innen in den Inszenierungen von Professionalität in Wechselwirkung von institutionellen Arrangements und sozialer Praxis zum Tragen kam: Die Proband/innen arbeiteten von Beginn an vor allem - auch bei offensichtlicher Überforderung - mit offenen individualisierenden Unterrichtsformen wie Tagesplan-, Wochenplan-, Werkstattformen und Gruppenarbeitsformen. Diese Formen verlangen hohe didaktische, methodische sowie psychologische Kompetenzen und ihre Erarbeitung und Etablierung mit und in der Klasse benötigt Zeit und klare strukturelle Vorgaben. Bei allen individuellen Unterschieden der Proband/innen zeigten sich folgende Schwerpunkte:

$9 \quad$ Teilstichprobe $(\mathrm{n}=10)$. 
- Etablierung eines effizienten Arbeitsstils und -rhythmus in den Vorbereitungen

- Etablierung von offenen Unterrichtsformen; Individualisierung

- Förderung der Sozialkompetenzen mit offenen Unterrichtsformen und -strukturen; Strukturierung des dynamischen Prozesses in der Klasse durch schüler/innenzentrierte Formen

- erzieherische, beraterische und begleitende Funktion der Lehrperson und flache Hierarchie in der Schüler/in-Lehrer/in-Beziehung

$\circ$ Engagement im Team - Schulhauskultur

- aktives und kompetentes Verhalten in Elternkontakten

○ bewusstes Nähe-Distanz-Management bezüglich der Öffentlichkeit

- Engagement im Kontakt zu Behörden und Fachleuten

$\circ \quad$ Engagement in der persönlichen Weiterbildung

$\circ$ bewusste Planung bzw. Balance der Arbeits- und Freizeit

Diese Schwerpunkte ergaben einen ersten Eindruck, wie und was die Proband/innen in ihrer Arbeit zu realisieren versuchten und wie sie sich als Lehrpersonen im Arbeitsfeld darstellten. Diese Elemente eines Selbstbildes veränderten sich im Verlaufe des ersten Praxisjahres, gemeinsame Themen blieben jedoch Führungsfragen, die Fragen nach Balance bzgl. Nähe und Distanz zu den Schüler/innen und den Eltern sowie die Herstellung und Darstellung von Sicherheit und Individualität in allen Kompetenzbereichen. Qualitative Unterschiede ließen sich in der Thematik von Nähe und Distanz zu den Schüler/innen der unterschiedlichen Schulstufen festhalten. Die Lehrerinnen und Lehrer stellten in diesem benannten Bereich starke Selbstansprüche an sich selbst und investierten einige Anstrengungen, um diese umsetzen zu können. Dies kann als Versuch gedeutet werden, die bestehenden Ideale, das pädagogische Konzept und die erlebte Arbeitsrealität in Einklang zu bringen. Dazu gehört auch, dass die meisten Lehrpersonen bei wahrgenommener Diskrepanz von persönlichen Zielen und der Unterrichtsrealität Maßnahmen einleiteten, die Friktionen durch produktive Anpassung oder Selbstverwirklichung kompatibler zu gestalten. Trotzdem beinhaltete der Bereich „Klassenführung“ vor allem für die männlichen Lehrpersonen negativ erlebte Handlungsmuster, die auf Konflikte verwiesen: Hier dominierten Disziplinierungs- und Führungsaufgaben die Wahrnehmung, welche mit Handlungsmustern wie „Schiedsrichter/in“, „Richter/in“ und „Polizist/in“ bewältigt wurden. Die weiblichen Lehrpersonen versuchten des Weiteren ihre Schüler/innen u.a. über Unterrichtsformen und -strukturen sowie Sozialformen so zu lenken, dass sie als Lehrpersonen nicht so stark gefordert waren und die Schüler/innen mehr Verantwortung übernehmen mussten. Zudem bauten sie bewusst und regelmäßig 
Zeitgefäße im Unterricht ein, in denen Kommunikations- und Interaktionsformen mit der Klasse besprochen und Regeln ausgehandelt wurden. Im Verlauf des ersten Praxisjahres suchten sie auch gezielt nach einer persönlichen Ausgestaltung von ungeliebten Handlungsmustern, oder überprüften aufgrund von inneren Konflikten ihre Ideale und Werte. Die befragten Lehrerinnen der Untersuchungsgruppe koppelten Führungsfragen denn auch gezielt an die „perfekte Vorbereitung“ und das Handlungsmuster „Vorbereitende“. Diese Inszenierung ergab eine äußerst bedeutsame Ressource wieder, weil damit Aspekte der Sicherheit verknüpft waren, die auf einen wahrgenommenen Anteil von Professionalität reagierten. Die erfolgreiche Umsetzung und Überprüfung der eigenen Vorstellungen in Unterrichtsformen und -strukturen verlieh den befragten Lehrerinnen zusätzlich Gewissheit über die eigene Kompetenz, die wiederum in den Interaktionen bestätigt wurde.

\section{„Das ist das schlimmste Jahr, das ich jemals erlebt habe“}

Probleme und Schwierigkeiten deuteten die männlichen Befragten ausschließlich auf der Folie von Professionskonzepten, nicht vor dem Hintergrund eines - in der Umkehrung der gängigen Formulierung - „doing work while doing gender". Sie brachten ihre beruflichen Ambivalenzen nicht in Zusammenhang mit Strukturmomenten des beruflichen Feldes, die den Rahmen für die intersubjektive Generierung von Bedeutungen bilden. Sie inszenierten sich zwar stimmig im Diskurs von beruflichen Konzepten und Idealen, jedoch inkonsistent zu den Vorgaben des beruflichen Feldes, und verorteten sich in der gängigen Opposition von Theorie und Praxis.

Meine Interpretation geht jedoch vor dem Hintergrund der eingangs geschilderten Perspektiven dahin, dass dieser Prozess aufgrund geschlechtsblinder Mitgliedschaftsentwürfe und als Folge von „doing gender“ im Kontext von „doing work“ gelesen werden kann, worauf exemplarisch ein Fall eines Berufsanfängers in der Studie verweisen soll: „Ich konnte nicht so unterrichten, wie ich mir das vorgestellt habe". Wiederholt äußerte er in den Interviews im Verlauf seines ersten Praxisjahres, dass er sich behaupten musste. Zum einen gegen eine Jungengruppe in der Klasse und gegen die Eltern seiner Schülerinnen und Schülern, die das plötzliche Aufbrechen von Gewalt in der Klasse, die seit drei Jahren gemeinsam den Unterricht besuchte, mit seinem professionellen Konzept und seinen Inszenierungen als männliche Lehrperson in Verbindung brachten. Dieser Lehrer erlebte, dass sich bestehende positive Erfahrungen und damit die eigene Subjektivität, nicht einfach von einem Raum (Ausbildung) auf den anderen (berufliches Feld) übertragen ließen. Die Räume waren - gerade bezüglich der Kategorie Geschlecht - nicht äquivalent o- 
der kompatibel strukturiert. Dieses Moment blendete der Lehrer konsequent aus, verweigerte sich Erwartungen, die er mit „Chef spielen“, „Polizist sein“ oder "Alphatier“ umschrieb und machte seine Unterrichtsprobleme daran fest, dass er etwa von „unrealistischen“ Kinderbildern ausgegangen sei.

Die professionellen Inszenierungen, in Wechselwirkung von institutionellen Arrangements und sozialer Praxis - so die These des vorliegenden Beitrages - stellen einen Balanceakt dar. Sie sind durch Spannungen gekennzeichnet und gelingen nicht immer zur persönlichen, institutionellen oder sozialen Zufriedenheit. Die befragten Lehrpersonen bezeichneten sich in verschiedenen Phasen in ihrem ersten Praxisjahr zwar grundsätzlich als Lernende, formulierten aber sehr deutlich den wahrgenommenen Anspruch, eindeutig und klar aufzutreten und ein verlässliches Gegenüber darzustellen. Selbst dann, wenn ihnen persönlich nicht deutlich war, was sie überhaupt in den verschiedenen Bereichen ihres Arbeitsfeldes erreichen wollten. Dazu kam erschwerend dazu, dass gegenüber Erwartungen Verhaltensweisen aufgebaut werden mussten, bevor eigene Entwürfe entwickelt werden konnten. Dies verdeutlicht, dass die Strukturen des beruflichen Feldes möglichst klar und transparent sein sollen, so dass Inszenierungen - „doing teacher“ - durch weibliche und männliche Lehrpersonen im Wissen um soziale Ordnungsmuster und institutionelle Reflexivität, um „Gender at work“ vollzogen werden können.

\section{Literatur}

Ashmore, Richrad D./Jussim, Lee (eds.) (1997): Self and Identity. Oxford

Bauer, Karl-Oswald/Kopka, Andreas/Brindt. Stefan (1996): Pädagogische Professionalität und Lehrerarbeit. Eine qualitative empirische Studie über professionelles Handeln und Bewusstsein. Weinheim

Bayer, Manfred et al. (2000): Lehrerin und Lehrer werden ohne Kompetenz? - Professionalisierung durch eine andere Lehrerbildung. Bad Heilbrunn

Combe, Arno/Helsper, Werner (1996): Pädagogische Professionalität. Frankfurt

Dann, Hanns-Dieter (1994): Pädagogisches Verstehen: Subjektive Theorien und erfolgreiches Handeln von Lehrkräften, in: Reusser, Kurt/Reusser-Weyeneth, Marianne (Hg.): Verstehen. Psychologischer Prozess und didaktisches Aufgabe. Bern

Dewe, Bernd/Radtke, Frank-Olaf (1993): Was wissen Pädagogen über ihr Können? Professionstheoretische Überlegungen zum Theorie-Praxis-Problem in der Pädagogik, in: Oelkers, Jürgen/Tenorth, Heinz-Elmar (Hg.): Pädagogisches Wissen. Weinheim 
Fenstermaker-Berk, Sarah (1985): The Gender-Factory: The Apportionment of Work in American Housholds. New York

Fend, Helmut (1980): Theorie der Schule. Frankfurt a.M.

Fend, Helmut (1998): Qualität im Bildungswesen. Weinheim

Frey, Hans-Peter/Hausser, Karl (Hg.) (1987): Identität: Entwicklungslinien psychologischer und soziologischer Identitätsforschung. Stuttgart

Giddens, Anthony (1988): Die Konstitution der Gesellschaft. Frankfurt a.M.

Goffman, Erving (1994): Interaktion und Geschlecht. Frankfurt a.M.

Gonon, Philipp (2003): Erziehung als Managementproblem: Bildungsinstitutionen zwischen Charisma und Taylorismus, in: Mangold, Max/Oelkers, Jürgen (Hg.): Ökonomie, öffentliche Bildung und Demokratie, Bern

Hausser, Karl (1983): Identitätsentwicklung. New York

Hausser, Karl (1995): Identitätspsychologie. Berlin

Heinz, Walter R. (1991): Berufliche und betriebliche Sozialisation, in: Hurrelman, Klaus/Ulich, Dieter (Hg.): Neues Handbuch der Sozialisationsforschung, Weinheim

Heinz, Walter R. (1995): Arbeit, Beruf und Lebenslauf. Eine Einführung in die berufliche Sozialisation. Weinheim

Hirsch, Gertrud (1990): Biographie und Identität des Lehrers. Eine typologische Studie über den Zusammenhang von Berufserfahrungen und beruflichem Selbstverständnis. Weinheim

Hoff, Ernst/Lappe, Wolfgang/Lappe, Lothar (Hg.) (1985): Arbeitsbiografie und Persönlichkeitsentwicklung. München

Hoff, Ernst (1990): Die doppelte Sozialisation Erwachsener. Zum Verhältnis von beruflichem und privatem Lebensstrang. München

Hoff, Ernst (1995): Frühes Erwachsenenalter: Arbeitsbiografie und Persönlichkeitsentwicklung, in: Oerter, Rolf/Montada, Leo (Hg.). Entwicklungspsychologie, Weinheim, 3. Auflage

Kelchtermans, Geert: Lehrer, ihre Karriere und ihr Selbstverständnis: Eine biografische Perspektive, in: Zeitschrift für Sozialisationsforschung und Erziehungssoziologie, Heft 12

Lenzen, Dieter (2003): Diagnose Lehrer. Plädoyer für die Professionalisierung eines Berufsstands, in: Universitats. Orientierung in der Wissenschaft

Mesmer, Beatrix (1988): Ausgeklammert-Eingeklammert. Frauen und Frauenorganisationen in der Schweiz des 19. Jahrhunderts. Basel und Frankfurt a.M.

Müller-Fohrbrodt, Gisela/Cloetta, Bernhard/Dann, Hanns-Dietrich (1978): Der Praxisschock bei jungen Lehrern. Formen-Ursachen-Folgerungen, Stuttgart

Oja, Sharon Nodie (1989): Teachers: ages and stages of adult development, in: Holly Mary Louise/McLoughlin, Caven S. (Eds.): Perspectives on Teacher Professional Development, London

Oser, Fritz (1997): Standards in der Lehrerbildung, Teil 1, in: Beiträge zur Lehrerbildung. 15. Jg., Heft 1 
Oser, Fritz (1997): Standards in der Lehrerbildung, Teil 2. Beiträge zur Lehrerbildung, in: 15. Jg. Heft 2

Rabe-Kleberg, Ursula (1996): Professionalität und Geschlechterverhältnis, in: Combe, Arno/Helsper, Werner (Hg.): Pädagogische Professionalität, Frankfurt a.M.

Schimank, Uwe (2000): Handeln und Strukturen. Eine Einführung in akteurtheoretische Soziologie, in: Zeitschrift für Lernforschung, 26. Jg., Heft 2

Schmitz, Gerdamarie S./Schwarzer, Ralf (1999): Proaktive Einstellung von Lehrern: Konstruktbeschreibung und psychometrische Analysen, in: Empirische Pädagogik, 13 (1)

Schmitz, Gerdamarie S. (1999): Zur Struktur und Dynamik der Selbstwirksamkeitserwartung von Lehrern. Ein protektiver Faktor gegen Burnout? Dissertation, Freie Universität Berlin

Schneider, Gerhard (1996): Lehrerkrisen und Supervision. Eine Studie zu Berufsanforderungen und zu einer Theorie der Lehrersupervision, Bad Heilbrunn

Schönknecht, Gisela (1997): Innovative Lehrerinnen und Lehrer. Berufliche Entwicklung und Berufsalltag, Weinheim

Schwarzer, Ralf/Schmitz, Gerdamarie (Hg.) (1997): Skalen zur Erfassung von Lehrerund Schülermerkmalen. Dokumentation der psychometrischen Verfahren im Rahmen der Wissenschaftlichen Begleitung des Modellversuchs Selbstwirksame Schulen. FU Berlin

Scott, Joan W. (1998): Gender und Politik, in: Waniek, Eva/Stoller, Sylvia (Hg.), Verhandlungen des Geschlechts, Wien

Straub, Jürgen (1998): „Personale und kollektive Identität. Zur Analyse eines theoretischen Begriffs“, in: Assmann, Aleida/Friese, Heidrun (Hg.): Identitäten, Frankfurt a.M.

Terhart, Ewald (Hg.) (1991): Unterrichten als Beruf. Neuere amerikanische Arbeiten zur Berufskultur und Berufsbiografie von Lehrern und Lehrerinnen, Köln

Wetterer, Angelika (1998): Integration und Marginalisierung. Das Verhältnis von Profession und Geschlecht am Beispiel von Ärztinnen und Juristinnen, Vortragsmanuskript FernUniversität - Gesamthochschule Hagen

Wetterer, Angelika (2002): Arbeitsteilung und Geschlechterkonstruktion. „Gender at Work" in theoretischer und historischer Perspektive. Konstanz 



\section{Geschlechterdifferenzen bei den Schulleistungen - empirische Forschungen aus England und Deutschland im Vergleich}

\section{Marita Kampshoff}

Geschlechterdifferenzen bei den Schulleistungen sind kultur- und kontextabhängig sowie historisch wandelbar. Dies zeigen nicht zuletzt die internationalen Schulleistungsstudien. Der Vergleich der an diesen Studien teilnehmenden Länder verdeutlicht, dass Geschlechterunterschiede bei den gemessenen Schulleistungen unterschiedlich groß sind. Sie sind nicht zwangsläufig und unvermeidbar. Es gelingt den Bildungssystemen einzelner Länder unterschiedlich gut, Mädchen und Jungen schulisch zu bilden.

In Schulleistungstudien werden mit Hilfe statistischer Verfahren zum Zusammenhang von Geschlecht und verschiedenen Aspekten wie Motivation, Schulform etc. Bedingungsfaktoren für die Differenzen angeschnitten. Eine vertiefende Analyse der Erklärungen für die Geschlechterdifferenzen bei den Schulleistungen verschiedener Länder, die die Rahmenbedingungen der unterschiedlichen Bildungssysteme und kulturelle und politische Bedingungen berücksichtigt, kann in den Studien nicht geleistet werden. Auch die Konsequenzen für das Bildungssystem oder gar den Schulalltag, die sich aus den Unterschieden bei den Schulleistungen ergeben, sind nicht leicht zu ziehen.

Aus diesem Grunde setzt die Forschungsarbeit am (empirisch untersuchten) Schulalltag an. Anhand einer Sekundäranalyse von empirischen Studien in diesem Gebiet wird überprüft, welche Erklärungsansätze sich für die Geschlechterdifferenzen bei den Schulleistungen finden. Neben dem Blick auf Deutschland werden auch englische Schulforschungen herangezogen. England bietet sich an, da hier zum einen seit Ende der 1980er Jahre Schulleistungen kontinuierlich und umfassend evaluiert werden und die Geschlechterdifferenzen ebenfalls erfasst werden. Zum anderen gibt es ein breites Feld von empirischen Forschungen zur Thematik, welches hierzulande kaum zitiert wird. 


\section{Methode des Vergleichs}

Das Ziel der Untersuchung ist es, herauszufinden, ob sich mit Hilfe der englischen Forschungsergebnisse Rückbezüge (vgl. Mitter 1996, S. 293ff.) für die deutsche Forschungslage zu den Begründungszusammenhängen der Geschlechterunterschiede bei den Schulleistungen finden lassen. Im Sinne der Vergleichenden Erziehungswissenschaften geht es bei Rückbezügen darum, Anstöße zur Verbesserung der eigenen Forschungen zu bekommen und eine theoretische Weiterentwicklung in diesem Gebiet voranzutreiben. Vergleiche sind fundamentale Wege, um Erkenntnisse zu gewinnen, zu erweitern und Erkenntnisniveaus zu erhöhen (vgl. Mitter 2001, S. 91). Vergleiche haben dabei verschiedene Funktionen. In der vorliegenden Arbeit stehen die idiographische Funktion - hier dient der Vergleich einem wissenschaftlich orientierten Interesse an der Spezifik des jeweiligen Gegenstandes - und die melioristische Funktion - hier dominiert der Wunsch, durch die Kenntnisse des ,anderen' Systems die eigene Praxis zu verbessern - im Vordergrund.

\section{Analyseraster}

Die einzelnen Aspekte, unter denen englische und deutsche Forschungen betrachtet werden, also das Analyseraster für den Vergleich, werden aus zwei in Deutschland entwickelten Modellen zu Bedingungen für schulisches Lernen und Leisten abgeleitet (Helmke/Weinert 1997 und Keller 1997).

Schulische Leistungen hängen nach einem Modell von Helmke und Weinert (1997) mit einer Reihe von Bedingungsfaktoren zusammen, die auf die Lern- und Leistungsergebnisse einwirken: Die soziale Herkunft der Lernenden, die Schule und der Klassenkontext, die Gleichaltrigen, die Lehrenden und die Unterrichtsprozesse sowie individuelle Lernvoraussetzungen und strategien werden hier genannt (zusammenfassende Darstellung des Modells nach Deutsches PISA-Konsortium 2001, S. 33). Bei diesem Modell wird die Geschlechtszugehörigkeit (ebenso wie das Lebensalter) als konstitutionelle Determinante der individuellen Lernvoraussetzungen betrachtet (vgl. Helmke/Weinert 1997, S. 103).

Carmen Keller hat 1997 ein Modell der Bedingungen für autonomes und andauerndes Lernverhalten entwickelt, welches sich mit dem Modell von Helmke und Weinert verbinden lässt. Keller unterscheidet externe und interne Variablen für das Lernverhalten. Extern sind: Klassenklima, LehrerInnen- 
SchülerInnen-Interaktion, Erwartung der Lehrperson, gesellschaftliche Stereotypisierung des Fachgebiets. Interne Variablen sind Interesse am Fachgebiet, Selbstvertrauen in eigene Fähigkeiten, praktischer Wert, der einem Fachgebiet beigemessen wird, Zuschreibung der Ursachen von Erfolg und Misserfolg sowie Übereinstimmung guter Leistungen mit der eigenen Geschlechtsrolle (vgl. ebd., S. 177). Bei Keller werden die Geschlechtsrolle als gesellschaftliche Verhaltenserwartung und die Geschlechterstereotypisierung eines Fachgebietes als Komponente für die Schulleistungen herangezogen. Die Kategorie Geschlecht wird somit neben der individuellen auf einer gesellschaftlichen Ebene angesiedelt.

In beiden Modellen finden sich eine Reihe von Anknüpfungspunkten für die Untersuchung der Geschlechterdifferenzen bei den Schulleistungen. Dabei werden auch weitere Determinanten/Variablen der Modelle von Helmke und Weinert sowie Keller einbezogen.

- Geschlecht als individuelle Determinante lässt sich aufsplitten als kognitive, motivationale und soziale Lernvoraussetzungen bei Mädchen und Jungen. Erfasst wird dies etwa bei den Selbstkonzepten der Lernenden sowie beim Zusammenhang zwischen Schulleistungen und der eigenen Geschlechtsrolle.

- In den subjektiven Theorien und Überzeugungen von Lehrenden können Geschlechterstereotypisierungen bei der Erwartung von guten oder schlechten Schulleistungen auftreten.

- Auch das Fachgebiet selbst, in dem Geschlechterdifferenzen bei den Schulleistungen auftreten, unterliegt Geschlechterstereotypisierungen. Physik bspw. gilt als ,männlich`.

- Ein breites Forschungsfeld stellen Schule, Klassenkontext, Altersgruppe u.v.m. in ihrer Wirkung auf die Geschlechterdifferenzen bei den Schulleistungen dar. Institutionelle Bedingungen der Schule wie auch Einflüsse der Peer-group-Kultur werden hier etwa erfasst.

- In den Unterrichtsprozessen, die in Interaktionen und Instruktionen aufschlüsselbar sind, zeigen sich unterschiedliche Lern- und Leistungsbedingungen für Mädchen und Jungen. Offen ist die Frage, welchen Einfluss dies auf die Schulleistungsunterschiede der Schülerinnen und Schüler hat.

- Der sozioökonomische Status und die ethnische Herkunft stellen für die Untersuchung der Geschlechterdifferenzen bei den Schulleistungen eine Differenzierungsmöglichkeit dar. Die Geschlechterverhältnisse unterscheiden sich je nach sozialer Herkunft und Migrationsgeschichte. 
In der Forschungsarbeit werden einige Aspekte dieses Modells überprüft und der Frage unterworfen, welche Erklärungsansätze sich in den empirischen Studien des jeweiligen Gebietes für die Geschlechterdifferenzen bei den Schulleistungen finden. Aus England werden in der Arbeite Studien zu den folgenden Vergleichsaspekten beleuchtet:

1. Aus dem Bereich ,Geschlecht als individuelle Determinante' Studien zu Selbsteinschätzungen, Fächervorlieben und der Geschlechtsrolle.

2. Bei der Überprüfung der subjektiven Theorien und Überzeugungen von Lehrenden zu den Geschlechterdifferenzen bei den Schulleistungen werden empirische Studien zu den LehrerInnenüberzeugungen zur Leistungsfähigkeit sowie zum Charakter, dem Verhalten und der Wahrnehmung der Persönlichkeiten von Schülerinnen und Schülern reanalysiert.

3. Auch die Unterrichtsprozesse stehen im Mittelpunkt vielfältiger Studien zu den Geschlechterdifferenzen bei den Schulleistungen. Neben dem konkret erforschten Instruktions- und Interaktionsgeschehen wird der Einfluss von geschlossenen und offenen Unterrichtskonzepten auf die Schulleistungen von Mädchen und Jungen sowie die Wirkung der Leistungsselektion auf Schülerinnen und Schüler überprüft.

4. Aus dem großem Feld ,Schule, Klassenkontext, Altersgruppe, Medienumwelt, elterliches Erziehungs- und Unterstützungsverhalten' liegen Ergebnisse zum Einfluss der englischen Bildungsreformen auf die Geschlechterdifferenzen bei den Schulleistungen vor. Studien zu mono- und koedukativen Schulen bzw. Klassen und dem Einfluss der Peers auf die Lern- und Leistungshaltungen von Jungen (und Mädchen) wird nachgegangen.

5. Als weiterer tragfähiger Bereich für die Erklärungsansätze für die Geschlechterdifferenzen bei den Schulleistungen zeigt sich die Leistungsmessung selbst. Hier werden die Diskussionen um externe oder schulinterne Messung der Schulleistung, Inhalte, Antwortstile und Materialien bei Examen und Tests sowie die Geschlechterfairness der PrüferInnen selbst ins Visier genommen. Dieser Aspekt wird in keinem der beiden Modelle berücksichtigt.

In Deutschland liegen ebenfalls Studien vor zu den Bereichen (1.) ,Geschlecht als individuelle Determinante' und (2.) ,subjektive Theorien von Lehrenden'. Weiterhin gibt es (3.) neuere, vor allem ethnographisch angelegte Interaktionsstudien sowie (4.) Untersuchungen, die den Einfluss mono- und koedukativer Unterrichtsorganisation auf die Geschlechterdifferenzen bei den Schulleistungen thematisieren. Insgesamt sind in Deutschland sehr viel weni- 
ger empirische Studien durchgeführt worden, die sich direkt mit den Geschlechterdifferenzen bei den Schulleistungen befassen.

In der rasant anwachsenden Anzahl von internationalen und nationalen Schulleistungsstudien, an denen Deutschland seit einigen Jahren vermehrt beteiligt ist, wird häufig die Geschlechtszugehörigkeit mit berücksichtigt. Die Geschlechterdifferenzen bei den Schulleistungen stehen hier allerdings nicht im Zentrum, sondern sind ein Aspekt unter vielen, die überprüft werden. Bei der Beschäftigung mit den Ursachen von Unterschieden zwischen Mädchen und Jungen wird oftmals auf Forschungsergebnisse anderer Länder eingegangen, ohne dass diese Studien kontextualisiert werden (etwa bei den Mathematikleistungen von Schülerinnen und Schülern). In der vorliegenden Forschungsarbeit wird eine solche Vorgehensweise nicht angewandt, da empirische Studien des einen Landes mit denen eines anderen verglichen werden.

\section{Exemplarische Ergebnisse}

Um den Ansatz der Arbeit zu veranschaulichen, stelle ich exemplarisch eines der eben genannten Gebiete vor - den Einfluss der Unterrichtsprozesse auf die Geschlechterdifferenzen bei den Schulleistungen. Wie die Modelle zu den Bedingungsfaktoren von Schulleistungen verdeutlichen - dies gleich vorweg -, verbieten sich monokausale Erklärungsansätze für das Erbringen oder Ausbleiben von Schulleistungen.

Unterrichtsprozesse und ihr Einfluss auf die Geschlechterdifferenzen bei den Schulleistungen in Deutschland

Es liegen in Deutschland eine Reihe differenzierter Ergebnisse zu Schulleistungsunterschieden bei Mädchen und Jungen vor (vgl. etwa Lehmann u.a. 1997, 1999, 2002; Baumert 1997, 2000; Deutsches PISA Konsortium 2001, 2003). In den Schulleistungstests wird der Unterricht selbst nicht auf die Lern- und Leistungsbedingungen für Mädchen und Jungen überprüft. Bei PISA werden aber beispielsweise die Lernstile von Schülerinnen und Schülern miteinander verglichen.

Viele Untersuchungen zu den Unterrichtsprozessen in Deutschland sind hingegen (noch) kaum auf Schulleistung fokussiert. In feministischen Interaktionsstudien wurde etwa untersucht, inwiefern Mädchen und Jungen am Unterrichtsgeschehen beteiligt werden und welche Wertschätzung ihnen dabei 
entgegengebracht wird (vgl. etwa Enders-Dragässer/Fuchs 1989; Frasch/ Wagner 1982). Dies stellt eine Rahmenbedingung für Lernen und das Erbringen von Schulleistungen dar. In einen konkreten Zusammenhang wurde dies allerdings nicht gebracht.

Zur Mädchenförderung in den Fachgebieten Mathematik, Technik und Physik wurden eine Reihe von Modellversuchen durchgeführt, in denen Unterrichtsprozesse eine Rolle spielten (vgl. etwa Nyssen 1996; Hoffman u.a. 1997). Hier zeigte sich, dass die Lernbedingungen der Schülerinnen, um Beispiele zu nennen, verbessert werden können, wenn Lehrkräfte sensibilisiert werden, Lehrund Unterrichtsmaterialien keine Geschlechterstereotypisierungen aufweisen und (tendenziell) verschiedene Interessensgebiete von Mädchen und Jungen im Unterricht gleichermaßen angesprochen werden. Inwiefern die Schulleistungen durch diese Modellversuche gesteigert werden konnte, wurde m.W. nicht überprüft. Es ging eher um allgemeine Rahmenbedingungen für das Unterrichten als um konkret gemessene Schulleistungen.

Bei den ethnographisch angelegten Studien, die Prozesse des, doing gender' im Schulalltag untersuchen, steht das Unterrichtsgeschehen bislang nicht im Vordergrund (vgl. Kelle/Breidenstein 1998; Faulstich-Wieland u.a. 2004). Dort, wo der Unterricht und das ,doing gender' in einen Zusammenhang gebracht werden (vgl. etwa Faulstich-Wieland 2002), lassen sich erste Schlüsse für die Lernbedingungen der Mädchen und Jungen ziehen: Es finden sich beispielsweise Hinweise darauf, dass Mädchen unterstellt wird, weniger mathematische Kompetenzen als Jungen zu haben oder dass bei den Schülern von derartigen Kompetenzen ausgegangen wird, ob sie diese nun haben oder nicht. Ebenso gab es aber auch Unterrichtssituationen, in denen Mathematik sinnvoll, verständlich und ohne auf Geschlechterstereotypisierungen Bezug zu nehmen vermittelt wurde. Weder in den Selbstkonzepten (vgl. FaulstichWieland 2002, S. 247ff.) noch in den Mathematikdurchschnittsnoten der Schülerinnen und Schüler (vgl. ebd. 2004, S. 50) kann jedoch ein deutlicher Niederschlag des jeweiligen Unterrichts nachvollzogen werden. Verallgemeinerbare Erklärungsansätze für die Geschlechterdifferenzen bei den Schulleistungen finden sich hier somit nicht.

\section{Unterrichtsprozesse und ihr Einfluss auf die Geschlechterdifferenzen bei den Schulleistungen in England}

In England liegen, neben anderen, den deutschen ähnlichen Studien, eine Reihe von Untersuchungen vor, die sich direkt mit dem Einfluss von Unterrichtsprozessen auf die Geschlechterdifferenzen bei den Schulleistungen be- 
fassen. In den Untersuchungsdesigns wird oftmals Methodentriangulation und Multiperspektivität angewandt. Das Zusammenspiel von Fragebogenerhebungen, Interviews mit Lehrenden, SchülerInnen, Unterrichtsbeobachtungen und Schulleistungstests ermöglicht vielschichtige Einblicke in das Unterrichtsgeschehen. Diese Vorgehensweise wird in Interaktionsstudien angewandt (vgl. Younger/Warrington/Williams 1999; Warrington/Younger 2000) oder beim Zusammenhang von Leistungsselektion und Gender (vgl. Gillborn/Kirton 2000; Boaler 1997a, b; siehe auch Kampshoff 2003, 2004). Auch die Unterrichtskonzepte stehen im Mittelpunkt derartig angelegter Studien (vgl. Boaler 1997a, 1998). Da der letztgenannte Punkt sich anbietet, in der gebotenen Kürze die Besonderheit dieses Designs darzustellen, veranschauliche ich an diesem, wie sich der Einfluss der Unterrichtsprozesse auf die Geschlechterdifferenzen bei den Schulleistungen zeigt.

Die Unterrichtsbeobachtungen und standardisierten Befragungen in Boalers Studien legen nahe, dass Schülerinnen und Schüler bei allen Ausnahmen tendenziell andere Lernstile (im von ihr erforschten Fach Mathematik) bevorzugen. An den beiden von ihr untersuchten Schulen werden verschiedene Unterrichtskonzepte umgesetzt, die unterschiedliche Lernstile begünstigen. Die Ergebnisse der von Boaler durchgeführten Schulleistungstests und der externen Examen zeigen signifikante Unterschiede zwischen den Schulen. Einer Reihe von Mädchen kommt es entgegen, Mathematik projektorientiert zu lernen, wie es die eine Schule verfolgt. Sie erbringen genauso gute Schulleistungen wie die Jungen dieser Schule. Jungen gelingt die Anpassung an den lehrgangsorientierten Unterricht der anderen jedoch besser als den Mädchen dieser Schule, sie haben deutlich höhere Werte bei den Schulleistungstests und Examen. Während die Schulleistungen der Schüler beider Schulen in etwa gleich ausfallen, sind die Schülerinnen der Schule mit projektorientiertem Unterricht leistungsstärker als im lehrgangsorientierten Unterricht der anderen Schule.

Gründe für die Leistungseinbußen bzw. -stärken der Schülerinnen und Schüler liegen auf verschiedenen Ebenen. Wie vor allem mit Hilfe qualitativer Interviews herausgearbeitet wurde, sind es bei zwei Gruppen schulinterne, bei einer dritten Gruppe eher gesellschaftliche Gründe, die Geschlechterdifferenzen bei den Schulleistungen begünstigen:

Auf der einen Seite finden sich eine relativ große Gruppe von Mädchen und einige Jungen, denen es beim lehrgangszentrierten und von der Lehrkraft dominierten Vorgehen daran fehlt, das, was sie lernen, auch zu verstehen und nicht nur anzuwenden. Auf der anderen Seite ist eine kleine Gruppe Jungen und vereinzelte Mädchen, die in vielen Fächern eine geringe Motivation zeigen, mitzuarbeiten und die sich überfordert fühlen, projektorientiert zu arbei- 
ten. Bei diesen Lernenden stimmt der Lernstil, den sie bevorzugen, nicht mit dem Unterrichtskonzept, nach welchem unterrichtet wird, überein. Wenn es im Unterricht gelingen würde, beide Lernstile zu berücksichtigen, können Mädchen wie Jungen gleichermaßen gute Schulleistungen erbringen ${ }^{1}$. Gelingt dies nicht, leistet die Gruppe weniger, deren Lernstil beim Mathematiklernen nicht berücksichtigt wird.

Für die dritte Gruppe, den Unterricht störende und unmotivierte SchülerInnen, scheint die Ursache für ihr Stören nicht in den jeweils angewandten Lehr/Lernmethoden zu liegen. Hier ist es eher die Motivation, lernen zu wollen, die einigen Schülern und wenigen Schülerinnen fehlt. Den Unterricht kritisierende Lernende der beiden ersten Gruppen zeigen Lernwillen und motivation im Mathematikunterricht, in der provokativen Gruppe fehlen diese. Hier scheint die Ursache eher im weiteren sozialen Umfeld als allein in der Schule gefunden werden zu müssen. Es könnten fehlende Berufs- oder Ausbildungsperspektiven die Ursache sein oder die ablehnende Haltung gegenüber Bildung im Elternhaus bzw. Freundeskreis sowie die präferierten Männlichkeiten/Weiblichkeiten der Peer groups. Bei diesen Lernenden wird in Frage gestellt, inwiefern Schulleistungen zu erbringen überhaupt als sinnvoll erachtet wird. Wenn dieser Sinn fehlt - und darauf deutet einiges hin -, können schulische Maßnahmen nicht mehr greifen. Derartige Ursachen für diese Geschlechterdifferenzen bei den Schulleistungen liegen nicht in der Schule selbst, zeigen dort aber nichts desto trotz einen gewichtigen Einfluss.

Die Ergebnisse von Boalers Studien müssten in weiteren Kontexten und Fächern überprüft werden.

\section{Fazit}

Wie kann nun ein Rückbezug der englischen Ergebnisse auf die deutsche Forschungssituation anhand dieses exemplarischen Ausschnittes aussehen? Wie sich gezeigt hat, ist die Forschungslage in den beiden Ländern divergierend. Aber vergleichen lässt sich nach Mitter (2001, S. 91) nicht nur Gleiches, sondern auch Ähnliches oder Verschiedenes. Es gilt lediglich, ein Vergleichskriterium zu finden - Hörner (1996, S. 13) nennt etwa den Saftgehalt von Äpfeln und Birnen. Das Vergleichskriterium ist in diesem Fall der Erklärungsgehalt,

1 Beiden SchülerInnengruppen könnte binnendifferenzierter Unterricht helfen, den verschiedenen Ansprüchen an die Lernstile und -wege gerecht zu werden. 
der für die Geschlechterdifferenzen bei den Schulleistungen in Studien zu Unterrichtsprozessen zu finden ist.

In Deutschland weisen Untersuchungsergebnisse darauf hin, dass Mädchen und Jungen unterschiedlich von den Lehrkräften wahrgenommen und am Unterricht beteiligt werden (vgl. etwa Nyssen 1996). Diese geschlechtsbezogenen Interaktionen wechseln sich - so zeigen neuere Studien (vgl. bspw. Faulstich-Wieland u.a. 2004) - aber auch mit Unterrichtssequenzen ab, in denen Geschlecht keine Rolle spielt.

In England liegen Ergebnisse verschiedener Studien vor, die den Zusammenhang zwischen Interaktionen, Aussagen von Lehrenden und Lernenden sowie den zu verschiedenen Zeitpunkten über Tests ermittelten Schulleistungen der Schülerinnen und Schüler überprüfen. Eine, hier ausgeführte, Studie zeigt einen Zusammenhang zwischen Lernstilen, unterschiedlichen Unterrichtskonzepten beim Mathematikunterricht und der erbrachten Schulleistung.

Während allgemein den Interaktionen des Unterrichts, auch wenn sie zeitweise von Geschlechterstereotypisierungen geprägt sind, kein hoher Erklärungsgehalt zukommt, ist die Verbindung Lernstile/Unterrichtskonzepte anscheinend für die Erläuterung der Geschlechterverhältnisse im (Mathematik)Unterricht ${ }^{2}$ tragfähig.

Das Ziel von Rückbezügen, Anstöße zur Verbesserung der eigenen Forschungen zu bekommen und eine theoretische Weiterentwicklung in diesem Gebiet voranzutreiben, lässt sich in erster Linie hinsichtlich der deutschen Forschungspraxis einlösen. Die theoretische Weiterentwicklung der Untersuchung von Unterrichtsprozessen für die Erklärung von Unterschieden in den Schulleistungen von Mädchen und Jungen könnte durch Multiperspektivität und Methodentriangulation, die Tests, standardisierte und offene Befragungen sowie qualitative Unterrichtsbeobachtungen verbindet, verbessert werden.

\section{Literatur}

Baumert, Jürgen et al. (1997): TIMSS - Mathematisch-naturwissenschaftlicher Unterricht im internationalen Vergleich. Deskriptive Befunde. Opladen

Baumert, Jürgen/Bos, Wilfried/Lehmann, Rainer (Hg.) (2000): TIMSS/III Dritte Internationale Mathematik- und Naturwissenschaftsstudie - Mathematische und na-

2 In Deutschland und Österreich wurde von Forscherinnen ebenfalls auf die Problematik des gängigen Unterrichtsstils in Mathematik für Mädchen hingewiesen (vgl. NiederdrenkFelgner 2001, Jungwirth 1994). Hinsichtlich der Schulleistung wurde dieser Zusammenhang $\mathrm{m} . \mathrm{W}$. jedoch nicht überprüft. 
turwissenschaftliche Bildung am Ende der Schullaufbahn. Band 2 Mathematische und physikalische Kompetenzen am Ende der gymnasialen Oberstufe. Opladen

Boaler, Jo (1997a): Experiencing school mathematics. Teaching styles, sex and setting. Buckingham/Philadelphia

Boaler, Jo (1997b): When even the winners are losers: evaluating the experiences of 'top set' students. In: Curriculum Studies, Vol. 29, Heft 2

Boaler, Jo (1998): Mathematical equity - underachieving boys or sacrificial girls? In: Epstein, Debbie/Maw, Janet/Elwood, Jannette/Hey, Valerie (Hg.): International Journal of Inclusive Education: Special Issue on Boys' Underachievement, 2. Jg. Heft 2

Breidenstein, Georg/Kelle, Helga (1998): Geschlechteralltag in der Schulklasse. Ethnographische Studien zur Gleichaltrigenkultur. Weinheim

Deutsches PISA-Konsortium (Hg.) (2001): PISA 2000. Basiskompetenzen von Schülerinnen und Schülern im internationalen Vergleich. Opladen

Enders-Dragässer, Uta/Fuchs, Claudia (1989): Interaktionen der Geschlechter. Sexismusstrukturen in der Schule. Weinheim

Faulstich-Wieland, Hannelore (2002): Welche Rolle spielen Lehrende und ihr Unterricht bei der Förderung von Mädchen in Mathematik? In: Kampshoff, Marita/ Lumer, Beatrix (Hg.): Chancengleichheit im Bildungswesen. Opladen

Faulstich-Wieland, Hannelore/Weber, Martina/Willems, Katharina (2004): Doing Gender im heutigen Schulalltag. Empirische Studien zur sozialen Konstruzktion von Geschlecht in schulischen Interaktionen. Weinheim

Frasch, Heidi/Wagner, Angelika (1982): „Auf Jungen achtet man einfach mehr...“. In: Brehmer, Ilse (Hg.): Sexismus in der Schule. Der heimliche Lehrplan der Frauendiskriminierung. Weinheim

Gillborn, David/Kirton, Alison (2000): White heat: racism, underachievement and white working-class boys. In: International Journa of inclusive Education Heft 4

Helmke, A./Weinert, F.E. (1997): Bedingungsfaktoren schulischer Leistungen. In: Weinert, Franz E. (Hg.): Psychologie des Unterrichts und der Schule, Göttingen

Hoffmann, Lore/Häußler, Peter/Peters-Haft, Sabine (1997): An den Interessen von Mädchen und Jungen orientierter Physikunterricht. Kiel

Hörner, Wolfgang (1996) ${ }^{4}$ : Einführung: Bildungssysteme in Europa - Überlegungen zu einer vergleichenden Betrachtung. In: Anweiler, Oskar u.a.: Bildungssysteme in Europa. Entwicklung und Struktur des Bildungswesens in zehn Ländern: Deutschland, England, Frankreich, Italien, Niederlande, Polen, Rußland, Schweden, Spanien, Türkei. Weinheim

Jungwirth, Helga (1994): Die Forschung zu Frauen und Mathematik: Versuch einer Paradigmenklärung. Journal für Mathematikdidaktik, 15, Heft $3 / 4$

Kampshoff, Marita (2004): Bildung als Schulleistung von Mädchen und Jungen -ein Überblick aus PISA, IGLU, TIMSS und Co. In: Betrifft Mädchen, 17. Jg. Heft 1

Kampshoff, Marita (2003): Was wissen wir über die leistungsschwachen Jungen aus PISA? Ein Blick nach England. In: Pädagogik Heft 2 
Keller, Carmen (1997): Geschlechterdifferenzen: Trägt die Schule dazu bei? In: Moser, U./Ramseier, E./Keller, C./Huber, M. (Hg.): Schule auf dem Prüfstand. Zürich

Lehmann, Rainer/Peek, Rainer (unter Mitarbeit von Rüdiger Gänsfuß) (1997): Aspekte der Lernausgangslage von Schülerinnen und Schülern der fünften Klassen an Hamburger Schulen. Bericht über die Untersuchung im September 1996. Behörde für Schule, Jugend und Berufsbildung, Amt für Schule (Hg.). Hamburg

Lehmann, Rainer/Peek, Rainer/Gänsfuß, Rüdiger/Husfeldt, Vera (2002): LAU 9. Aspekte der Lernausgangslage und der Lernentwicklung - Klassenstufe 9 - Ergebnisse einer längsschnittlichen Untersuchung in Hamburg. Hamburg

Lehmann, Rainer/Peek, Rainer/Gänsfuß, Rüdiger (1999): Aspekte der Lernausgangslage und der Lernentwicklung von Schülerinnen und Schülern an Hamburger Studien - Klassenstufe 7. Bericht über die Untersuchung im September 1998. Behörde für Schule, Jugend und Berufsbildung, Amt für Schule (Hg.). Hamburg

Mitter, Wolfgang (2001): Der Vergleich in der Erziehungswissenschaft und die Vergleichende Erziehungswissenschaft. Oskar Anweiler zum 75. Geburtstag. In: Bildung und Erziehung, Heft 1

Mitter, Wolfgang (Hg.) (1996): Wege zur Hochschulbildung in Europa. Vergleichsstudie zum Verhältnis von Sekundarabschluß und Hochschulzugang in Frankreich, England und Wales, Schweden und Deutschland. Frankfurt a.M.

Niederdrenk-Felgner, Cornelia (2001): Die Geschlechterdebatte in der Mathematikdidaktik. In: Hoppe, Heidrun/Kampshoff, Marita/Nyssen, Elke. Geschlechterperspektiven in der Fachdidaktik. Weinheim

Nyssen, Elke (1996): Mädchenförderung in der Schule. Weinheim

Warrington, Molly/Younger, Mike (2000): The other side of the gender gap. In: gender and Education, Vol. 12, Heft 4

Younger, Mike/Warrington, Molly/Williams, Jaquetta (1999): The Gender Gap and Classroom Interactions; reality and rhetoric? In: British Journal of Sociology of Education, Vol. 20, Heft 3 



\section{„Action is indeed the sole medium of expression for ethics" Skizzen einer sozialen Ethik bei Jane Addams Inga Pinhard}

Als Jane Addams im Jahre 1900 auf das Podium der New York Society for Ethical Culture trat, kreiste ihr Vortrag The Social Test um das Spannungsfeld zwischen den abstrakten theoretischen Ansprüchen moralischer Konzeptionen auf der einen und konkretem moralischen Handeln in der Praxis auf der anderen Seite. „We continually forget that the sphere of morals is the sphere of action, that speculation in regard to morality is but observation and must remain in the sphere of intellectual comment, that a situation does not really become moral until we are confronted with the question of what shall be done in a concrete case, and are obliged to act upon our theory“ (Addams 1900, S. 398). Erst im sozialen Handeln erweist sich die Relevanz von Theorien, so die These der Sozialreformerin und -theoretikerin Addams (1860-1935), die Ende des 19. Jahrhunderts zunächst als Gründerin des Settlements Hull Hou$s e$ in Chicago und später als international agierende Pazifistin, Pädagogin und Aktivistin für Menschen- und Frauenrechte berühmt und 1931 mit dem Friedensnobelpreis ausgezeichnet wurde. ${ }^{1}$ Das Verhältnis von Theorie und Praxis neu zu denken, stereotype Dualismen aufzulösen und der Vorsatz, eine philosophische Theorie zu entwickeln, deren Ziel es ist, alle Mitglieder einer Gesellschaft zu ermächtigen, diese gleichberechtigt mitzugestalten, zeichnen ihren Entwurf einer sozialen Ethik aus. Sie vereint wesentliche Merkmale pragmatistischer und feministischer Theorien und nimmt Aspekte vorweg, die bis heute die feministische Kritik an normativen Moraltheorien prägen; dazu gehört die Forderung nach Anerkennung der Kontextbezogenheit von Moral und moralischen Urteilens, verbunden mit einem Verständnis des Menschen als Teil eines interagierenden, interrelationalen Beziehungsnetzwerks und nach Betonung der reziproken Abhängigkeit der Menschen. Demnach muss auch die Relevanz von Emotionen, Bindungen und Beziehungen für moralisches Handeln neu bewertet werden. Feministische Ethik, verstanden als „gender-bezogene Analyse der Moralphilosophie und konkreter moralischer

1 Zur Biographie von Jane Addams verweise ich auf die exzellenten Monographien von Jean Bethke-Elshtain (2002b), Victoria Bissell Brown (2004), Christopher Lasch (1965) und auf die historische Studie über Jane Addams und Alice Salomon von Anja Schüler (2004). 
Probleme“ (Pauer-Studer 2003, S. 109), und der amerikanische Pragmatismus, beide keine geschlossenen, homogenen Theoriemodelle, bieten den primären Bezugsrahmen für die anschließenden Überlegungen. Charlene Haddock-Seigfried begreift Feminismus und Pragmatismus als Interessengemeinschaft im Ringen um eine „Re-vision“ der Philosophie „within a reformation of education more generally within a renovation of society at large" (Seigfried 1993, S. 1).

Dieser Artikel gewährt einen kleinen Einblick in mein Dissertationsprojekt, in dem ich mich mit Jane Addams und ihren Perspektiven für Theorien ethischer und ästhetischer Bildung und Erziehung beschäftige; ein Projekt, das weniger deshalb einen Beitrag zur pädagogischen Geschlechterforschung leisten soll, weil es, in der Tradition „klassischer“ Frauenforschung, kompensatorisch das bisher innerhalb der deutschen Erziehungswissenschaft eher selektiv rezipierte Werk einer außergewöhnlichen Denkerin thematisiert. Die Konstruktion einer neuen Klassikerin kann dabei allenfalls ein positiver Nebeneffekt sein. Mein Anliegen ist vielmehr, anhand konkreter Fragestellungen die Verflechtungen und Wechselwirkungen ihrer pädagogischen und philosophischen gesellschaftskritischen Konzepte mit zeitgenössischen Diskursen in Philosophie, Pädagogik, Literatur und Politik zu rekonstruieren und kritisch zu beleuchten, um ihre Ansätze und Positionen, in einem nächsten Schritt, in Dialog zu bringen mit gegenwärtigen sozialphilosophischen und erziehungswissenschaftlichen Debatten um Pragmatismus, feministische Theorie, ästhetische Bildung, Ethik und eine Pädagogik der Anerkennung. ${ }^{2}$ Zum einen geht es also darum ihr Werk in seinem spezifischen historischen, soziokulturellen Kontext zu situieren und zu diskutieren, dass heißt in den USA, in Chicago zur Zeit der Progressive Era, und zum anderen darum zu überlegen, welche Impulse ihre Konzepte und Projekte aktuellen erziehungswissenschaftlichen Fragestellungen geben können.

Das konstitutive Moment der sozialen Ethik von Jane Addams ist die Prämisse, dass Moral gebunden ist an soziales Handeln, die Möglichkeit zu handeln aber nicht allen Mitgliedern der Gesellschaft gleichberechtigt gegeben ist, sondern wesentlich von Faktoren wie Geschlecht, Klasse, Rasse, Bildung, Erziehung und ökonomischen Verhältnissen abhängt. Die Diskrepanz zwischen moralischen Idealen und Theorien und moralischem Handeln werde ich anhand zweier Aspekte diskutieren: Die moralische Dimension der Literatur als Medium und potentiellem Impuls für die Auseinandersetzung mit sozi-

2 Aktuelle Bezüge werden unter anderem hergestellt zu den Theorien von Seyla Benhabib, Jessica Benjamin, Judith Butler, Micha Brumlik, Axel Honneth, Annedore Prengel und Isabell Diehm. 
alen Bedingungen und Konflikten steht im Mittelpunkt des ersten Abschnitts. Welchen Einfluss kann Literatur auf moralisches Handeln haben, wo liegen Chancen und Grenzen? Um dies zu klären, werden Addams' Überlegungen im Spiegel des Literaturverständnisses von Walt Whitman, dem umstrittenen Dichter, der als Prophet der amerikanischen Demokratie berühmt wurde, erörtert. Addams und Whitman eint die Vision einer Demokratie, pragmatistisch gedacht als ,system of interrelated and mutually modifying values, ideas, and imperatives to act" (Mack 2002, S. 164), eine Demokratie in der alle gleichberechtigt partizipieren. Die Forderung nach einem Wandel, einer Demokratisierung der Geschlechter- und Generationenverhältnisse ist für Addams und Whitman eine notwendige Bedingung für die neue demokratische Gesellschaft. Am Beispiel des Geschlechter- und Generationenkonflikts zwischen Töchtern und ihren Familien, der eng verbunden ist mit einer Kritik an einer Erziehungspraxis, die moralische Konzepte und Theorien vermittelt ohne einen Handlungsbezug herzustellen, wird in einem Ausblick angerissen, wie Addams in ihrem Entwurf einer sozialen Ethik individuelle und soziale Moral herleitet und im Hinblick auf die Rolle von Nahbeziehungen für Moralentwicklung problematisiert.

\section{Literatur und Moral - Wechselspiele}

Die Reflexion über die Wechselbeziehung von Literatur, Moral und sozialem Handeln ist ein immer wiederkehrendes Motiv in den Schriften und Vorträgen von Jane Addams. Literatur wird in ihrem Werk in unterschiedlichen Funktionen wirksam, besitzt aber immer eine moralische Komponente. Sie verwendet Analogien aus literarischen Werken bei der Analyse von sozialen Konflikten, wie beispielsweise in ihrem Essay A Modern Lear (1894), ihrer radikal sozialkritischen Stellungnahme zum Pullmanstreik in Chicago, in der sie die Analogie zwischen den Beziehungsverhältnissen von Shakespeares König Lear und seiner Tochter Cordelia und Pullman und seinen Arbeitern als Basis verwendet, um die moralischen Defizite eines falsch verstandenen Generationen- und Geschlechterverhältnisses auf der einen und eines ,paternalistischen Wohlfahrtskapitalismus“ (Westbrook 1991, S. 88 Übersetzung I.P.) auf der anderen Seite zu demaskieren. Beide Male, so ihre These, wird das menschliche Bedürfnis nach Autonomie und gleichberechtigter sozialer Partizipation negiert, was zwangsläufig zu einschneidenden Konflikten führen muss. Addams versteht Literatur als Medium von Sozialkritik, als Anregung zur Refle- 
xion und spricht ihr neben einem moralischen auch ein hohes bildendes und erzieherisches Potential zu. Doch ihrem Verständnis nach sind Bildung und Erziehung, wie auch Moralentwicklung, immer gebunden an unmittelbare Erfahrungen und soziales Handeln. Literatur kann und soll zu einer Veränderung, einer Erneuerung aufrufen, aber ohne direkten Handlungsbezug muss sie der Abstraktion, der Ebene von Idealen und Theorien verhaftet bleiben. „Literary light on social questions is hurtful or beneficial, as it enervates or spurs to action. Strong expression is interesting but not definite. We need contact, experience, the doing things in common. Until we lose individual good in desire for the good of the whole, we do not reach morality, and this enlarged life comes to us through enlarged experience" (Addams 1900, S. 398).

Skeptisch beurteilt Addams die Neigung ihrer Generation, sich mit sozialen Problemen nicht unmittelbar durch Handeln, sondern vermittelt durch Literatur und literarischen Ausdruck auseinander zu setzen und damit die Kluft zwischen Theorie und Praxis weiter zu perpetuieren. Da sich Moral erst im moralischen Handeln ausdrückt, kann der Einfluss der Literatur nur indirekt sein. Addams erkennt bei den Literaten wie bei den LeserInnen ein gesteigertes Interesse an sozialen Fragen und ein Bedürfnis nach Veränderung. Sozialund Gesellschaftskritik sind in Mode, kein Roman wird zum Bestseller ohne soziale Fragen zu thematisieren. „No novel excites great attention unless it presents social questions and the public apparently feels that it enters into complicity by reading the work, and is, in some occult way, helping on the good cause" (Addams 1900, S. 398). Sie beanstandet, dass die Lektüre, die intellektuelle Beschäftigung mit sozialer Ungleichheit und sozialen Konflikten zunächst keine Konsequenzen für die Praxis zu haben scheint, da durch den Akt des Lesens bereits ein Gefühl der Partizipation entsteht. Der Wirkung der Literatur sind Grenzen gesetzt, ihre Aufgabe ist das Enthüllen, die Reund Dekonstruktion gesellschaftlicher Prozesse, Praktiken, Ideologien, Machtverhältnisse und daraus resultierenden Ungleich- und Ungerechtigkeiten. "The business of literature is revelation and not reformation" (Addams 2002b, S. 8). Doch ist Enthüllen und Aufdecken nicht immer verbunden mit Umgestaltung und Erneuerung? Unterschätzt Addams die Wirkung und den subversiven Charakter von Literatur?

Der Poet Walt Whitman (1818-1892) plädiert dafür, den moralischen Einfluss von Literatur nicht zu unterschätzen, und geht, wie Stephen Mack in seiner Studie The Pragmatic Whitman ausführt, optimistisch von einer Durchdringung und Unvermeidlichkeit der moralischen Funktion von Literatur aus. Diese moralische Funktion ist nicht daran zu messen, ob die LeserInnen den moralischen Appell Gutes zu tun ignorieren oder befolgen, sondern 
liegt darin, dass Literatur die gesamte geteilte Epistemologie einer Zivilisation übermittelt (Mack 2002, S. 139). Das Potential der Literatur für Moralentwicklung wird von dem Literaten und der Sozialreformerin unterschiedlich hoch bewertet, was mit daran liegen mag, dass sie aus unterschiedlichen Perspektiven sprechen, ihre Anliegen und Visionen treffen sich jedoch nicht nur in einem gemeinsamen Demokratieverständnis. Beide verweisen auf die Kontextualität von Literatur, Moral, Wissen, Bildung und Erziehung, die nie isoliert, sondern immer historisch situiert und eingebunden in gesellschaftliche und kulturelle Prozesse betrachtet werden müssen und stetigem Wandel unterliegen. „The virtues of one generation are not sufficient for the next, any more than the accumulations of knowledge possessed by one age are adequate to the needs of another" (Addams 1894 in: Elshtain 2002a, S. 170). Für Whitman muss am Beginn einer neuen Gesellschaft eine neue Literatur stehen, ${ }^{3}$ die über die Ebene der Reflexion, der Re- und Dekonstruktion gesellschaftlicher Prozesse herausgeht, deren Aufgabe es ist ,not merely to copy and reflect existing surfaces, or pander to what is called taste - not to amuse, pass away time, celebrate the beautiful, the refined, the past, or exhibit technical, rhythmic, or grammatical dexterity - but a literature underlying life, religious, consistent with science, handling the elements and forces with competent power, teaching and training men" (Whitman 1963, S. 372). An der Frage nach der moralischen Funktion von Literatur in Erziehungs- und Bildungsprozessen hat sich die westliche Sozial- und Bildungstheorie seit Platon und Aristoteles abgearbeitet. Literatur darf für Whitman nicht als Zeitvertreib, Flucht aus der Realität oder technische und stilistische Spielerei verstanden werden, sondern muss immer Bezug zum Leben der Menschen haben, aller Menschen, unabhängig von Geschlecht, Klasse oder Ethnizität. Sie muss sich von den Normen des Geschmacks befreien, der sie dazu drängt, nur das vermeintlich Schöne und Edle zu feiern. Denn was ist schön und edel, richtig oder falsch, wer definiert das mit welcher Legitimation? Whitmans Literaturverständnis hat entscheidende Auswirkungen auf den Bildungs- und Erziehungsanspruch literarischer Werke, denn diese sollen nicht mehr in der Tradition moralisierender Erziehungsromane und Erziehungsratgeber des 18. und 19. Jahrhunderts gesellschaftliche Normen und Konventionen transportieren und zementieren, sondern diese vielmehr zur Disposition stellen und zum selbständigen

3 Whitman weist der Poesie in der amerikanischen Demokratie eine entscheidende Rolle zu und versteht den Dichter als Vorreiter, als Führer unter Führern: „For the great idea, the idea of perfect and free individuals, for that the bard walks in advance, leader of leaders, the attitude of him cheers up slaves and horrifies foreign despots" (Whitman: By Blue Ontario's Shore zitiert nach Nussbaum 2003, S. 645) 
Denken und Selbsttätigkeit anregen. ${ }^{4}$ Genau an diesem Punkt treffen sich Whitman und Addams. Whitmans Werk ist die literarische Vision einer Demokratie, einer demokratischen Gesellschaft, die durch radikale Reform Wirklichkeit werden kann, eine Vision, die eine „Grammatik“, einen Rahmen bietet für Sozial- und Kulturkritik (Mack 2002, S. 164), als Voraussetzung für Wandel und Erneuerung. Diese Vision von Demokratie als Lebensform teilt Jane Addams und überträgt sie auf soziale Praxis. Addams und Whitman erweisen sich in ihren Schriften als zutiefst pragmatistische DenkerInnen. Stuart Pratt identifiziert in seinem Buch Native Pragmatism vier wesentliche Charakteristika von Theorien des amerikanischen Pragmatismus, die sich auch bei Addams und Whitman finden: ein Verständnis von Interaktion, das Erfahrung nicht als etwas begreift, das passiven Subjekten passiert, sondern als Mittel zur aktiven gesellschaftlichen Partizipation; die Akzentuierung eines ontologischen, epistemologischen und kulturellen Pluralismus; ein Konzept von Gemeinschaft, dass das Spannungsverhältnis zwischen Individuum und Gesellschaft anerkennt und neu definiert; und schließlich der Glaube an Wachstum, verbunden mit der Prämisse, dass erst die Chance zu individuellem Wachstum und gleichberechtigter demokratischer Partizipation, zum Wachstum der Gemeinschaft führen kann (vgl. Hamington 2004, S. 94f.). Im Folgenden wird exemplarisch am Konflikt graduierter Frauen gezeigt, wie wichtig der Zugang zu einer Vielfalt von Erfahrungen, gleichberechtigte gesellschaftliche Partizipation und damit zwingend ein Wandel der Geschlechterverhältnisse für die Entwicklung einer sozialen Moral als Fundament einer Demokratie sind. „To follow the path of social morality results perforce in the temper if not the practice of the democratic spirit, for it implies that diversified human experience and resultant sympathy which are the foundation and guarantee of Democracy“" (Addams 2002a, S. 7).

4 Damit steht er im Kontext einer literarischen Entwicklung, wie sie Mitte des 19 Jahrhunderts Waldo Emerson, Henry David Thoreau oder Harriet Beecher-Stowe in den USA vorantrieben. Whitmans Werk, angefangen mit der prophetischen Lyrik der Leaves of Grass (1855) bis hin zur komplexen Prosa der späteren Essays wie Democratic Vistas (1871), entstand in einer Zeit der Aufbruchsstimmung und starken gesellschaftlichen und kulturellen Wandels, ebenso geprägt von Industrialisierung, Technisierung, Urbanisierung und Sezessionskrieg, wie von Auseinandersetzungen um die Abschaffung der Sklaverei, um gleiche Bürgerrechte für alle Mitglieder der Gesellschaft und um Forderungen der Frauenbewegung. 
Individuelle und soziale Moral Ambivalenzen, Ein- und Ausblicke

„Democracy, in silence, biding its time, ponders its own ideals, not of literature and art only - not of man only, but of women. The idea of the women of America developed, raised to become the robust equals, workers, and, it may be even practical and political deciders with the man" (Whitman 1963, S. 389). Als Whitman 1871 diese Zeilen schreibt, ist zwar die Gründung zahlreicher privater höherer Schulen, Colleges und Universitäten für die Töchter der weißen Ober- und Mittelschicht eine der wichtigsten Errungenschaften der Frauenbewegung, aber dennoch haben Frauen kein Wahlrecht, der Zugang zu Universitäten ist eingeschränkt und nur wenige Colleges und Universitäten sind koedukativ. ${ }^{5}$ Noch um 1900 sahen sich Absolventinnen nach ihrer Graduierung, die oft mit einer Rückkehr in die Familie verbunden war, schnell mit der Frage konfrontiert, was denn nun tun mit der erlangten Bildung und Erziehung. Diesen Konflikt reflektiert Jane Addams in einer vehementen Kritik am Erziehungssystem, an den Inhalten der schulischen Mädchenbildung, der strikten Trennung theoretischer Konzepte von sozialer und gesellschaftlicher Praxis und einem antiquierten Familien- und Frauenideal, das Frauen nicht als gleichberechtigte Familienmitglieder, als selbstverantwortliche Individuen, sondern als Familienbesitz betrachtet (Addams 2002, 39f.). Diese ideologische Ausgrenzung von Frauen aus dem realen Leben ist ethnizitäts- und schichtspezifisch und orientiert sich an einem Frauenideal der privilegierten weißen Mittel- und Oberschicht, das bestehende patriarchale Machthierarchien reproduziert. Mit Whitman teilt Addams die Überzeugung, dass eine Demokratisierung und Umstrukturierung der Geschlechter- aber auch der Generationenverhältnisse - sei es in Familie, Staat und Institutionen, wie Schule oder Universität - unverzichtbar für gesellschaftlichen Wandel sind.

„The young people $[. .$.$] have ideas and theories of life too big to swing,$ and so are perforce reduced to the literary, or looking on ranks. [...] I'm impressed by the charming young girls who want to come into my settlement work. They have delightful manners and theories, but they have known only one sort of people. Their training has been essentially narrow, aristocratic,

5 Das Oberlin College ist ab 1833 das erste koedukative College in den USA. Einen systematischen Überblick über die Geschichte der Frauenbildung in den USA bietet Barbara Miller Solomon (1985). Ausschlüsse vom Zugang zu Bildung gründen in sozialer Herkunft, Klasse und Ethnizität - Kriterien, die die Machtverhältnisse in der Gesellschaft re-produzieren. Geschlecht ist ein verstärkender Faktor, ähnliche Exklusionen sind aber auch im Bereich der Jungenbildung nachweisbar. 
when they come into contact with other classes, their social training utterly breaks down" (Addams 1900, S. 398). Die Tradition, Frauen im Sinne einer wie ich an anderer Stelle aufgezeigt habe ${ }^{6}$ - nicht durchhaltbaren Separate Spheres-Ideologie, an einen privaten, häuslichen Schonraum zu binden, und ihnen damit plurale Erfahrungen zu verwehren, perpetuiert das Spannungsfeld zwischen theoretisch fundierten moralischen Ansprüchen und konkretem Handeln. Dadurch wird das von Addams beklagte Missverhältnis von individueller und sozialer Moral bezogen auf soziales, gesellschaftliches und politisches Handeln wesentlich beeinflusst. In ihrem Entwurf einer sozialen Ethik sind die wechselseitigen Interaktionen und Verpflichtungsverhältnisse innerhalb der Familie und der primären Bezugsgruppen konstitutiv für individuelle Moral, viel bedeutender ist jedoch eine umfassende soziale Moral als Fundament von Demokratie. Moralische Standards, die dem Bereich konkreter persönlicher Beziehungen verhaftet bleiben, sind nicht auf alle sozialen Verhältnisse übertragbar, sie sind viel mehr entscheidend für eine individuelle als für die von Addams eingeforderte soziale Moral. Die Qualität und die Vielfalt von Beziehungen und Erfahrungen haben signifikanten Einfluss auf die Entwicklung von sozialer Moral. Erst durch das Wissen um und über einander im sich ins Verhältnis setzten im Sinne Benhabibs nicht nur zu dem konkreten, sondern auch zu dem verallgemeinerten Anderen - sind die Anteilnahme, die Achtung, die wechselseitige Anerkennung möglich, die für Addams moralisches und soziales Handeln bedingen. „Vom Standpunkt des verallgemeinerten Anderen ist jeder einzelne eine moralische Person mit gleichen moralischen Rechten wie wir selbst; diese Person ist auch ein argumentierendes und handelndes Wesen, fähig, einen Sinn für Gerechtigkeit zu entwickeln, festzulegen was es für moralisch gut hält und dementsprechend zu handeln. Die Vorstellung vom konkreten Anderen hingegen lässt uns jede moralische Person als einzigartiges Individuum mit einer ganz bestimmten Lebensgeschichte, bestimmten Veranlagungen und Begabungen, Bedürfnissen und Grenzen sehen" (Benhabib 1992, S. 19). Mit diesen Überlegungen führt Benhabib aus, was bei Addams in der Forderung nach einer Vielfalt von direkten Erfahrungen und nach gesellschaftlicher Partizipation, die eine Konfrontation mit konkreten Anderen und dadurch ein sich-ins-Verhältnis-Setzen der einzelnen Individuen erst möglich macht, angerissen wird. Während John Dewey Familie und Nachbarschaft als wesentliche Quelle für moralische Haltungen und moralisches Handel interpretiert, da sie „bei all ihren Fehlern, immer die Haupt-

$6 \mathrm{Zu}$ konkurrierenden Frauenbildern und ideologischen Konzepten von Weiblichkeit um 1900 vgl. Inga Pinhard (2005). Diskutiert wird in diesem Aufsatz weiterhin das oft stereotyp geschlechtlich markierte Verhältnis von Privatheit und Öffentlichkeit(en), das sich im 19. Jahrhundert wesentlich verschiebt. 
kräfte der Erziehung, die Mittel [sind], durch die Anlagen ausgeprägt und Ideen erworben werden, welche Wurzeln im Charakter schlagen" (Dewey 2001, S. 175), sieht Addams die Rolle dieser Nahbeziehungen für Moralentwicklung eher ambivalent. Da sie sich sowohl praktisch, empirisch, wie auch theoretisch intensiv mit dem Konfliktpotential von diesen Beziehungsverhältnissen auseinandersetzt, fehlt ihr Deweys Optimismus im Glauben an „die Erneuerung gemeinschaftlichen Lebens durch die Demokratisierung von Familie und Heimatgemeinde" (Brumlik 2002, S. 267). In dieser Skepsis nimmt sie Ambivalenzen vorweg, die bis heute die feministische Debatte um Moralphilosophie und Ethik beschäftigen. Der moralische Wert von Nahbeziehungen und persönlichen Bindungen wird von zahlreichen Theorien feministischer Ethik betont ${ }^{7}$ und gleichzeitig in einer Debatte um Partikularismus und Universalismus problematisiert. Schließen möchte ich mit einem Ausblick von Seyla Benhabib, der mir sowohl fruchtbar für Addams' Entwurf einer soziale Ethik als Ethik der Anerkennung zu sein scheint, wie auch für aktuelle erziehungswissenschaftliche und feministische Theorien von Ethik und Moral: „Will man Universalisierbarkeit als ein Umkehren der Perspektive definieren und als Bemühen, den Standpunkt des oder der anderen zu verstehen, so darf man diese anderen nicht nur als allgemeine Andere denken, man muss sie auch als konkrete andere wahrnehmen“"(Benhabib 1992, S. 18).

\section{Literatur}

Addams, Jane (1900): The Social Test. In: Public Opinion 28 (29. März 1900).

Addams, Jane (2002a): Democracy and Social Ethics. Chicago.

Addams, Jane (2002b): A New Conscience and Ancient Evil. Chicago.

Benhabib, Seyla (1995): Selbst im Kontext. Frankfurt am Main.

Brown, Victoria Bissell (2004): The Education of Jane Addams. Philadelphia.

Brumlik, Micha (2002): Bildung und Glück. Versuch einer Theorie der Tugenden. Berlin/Wien.

7 Feministische Debatten um Ethik wurden in den 1980er Jahren wesentlich beeinflusst durch Carol Gilligans entwicklungspsychologische Untersuchungen zur Moralentwicklung von Frauen. Gilligans Unterscheidung zwischen zwei konträren Moralkonzeptionen, nämlich der männlichen Orientierung an Gerechtigkeit und Rechten und der weiblichen Orientierung an affektiven sozialen Bindungen, Fürsorge und Anteilnahme (Carol Gilligan (1984): Die andere Stimme. München), führte zu zahlreichen Entwürfen von Care Ethiken (vgl. beispielweise Nel Noddings (1984): Caring. A feminine Approach to Ethics and Moral Education. Berkeley oder Sara Ruddick (1993): Mütterliches Denken. Für eine Politik der Gewaltlosigkeit. Frankfurt am Main oder Elisabeth Conradi (2001): Take Care, Frankfurt a.M.). 
Dewey, John (2001): Die Öffentlichkeit und ihre Probleme. Berlin/Wien. Elshtain, Jean Bethke (Hg.) (2002a): The Jane Addams Reader. New York.

Elshtain, Jean Bethke (Hg.) (2002b): Jane Addams and the Dream of American Democracy, New York.

Hamington, Maurice (2004): Embodied Care. Jane Addams, Maurice Merleau-Ponthy and Feminist Ethics. Urbana/Chicago.

Lasch, Christopher (1965): The Social Thought of Jane Addams. Indianapolis.

Mack, Stephen John (2002): The Pragmatic Whitman. Reimagining American Democracy. Iowa City.

Nussbaum, Martha (2003): Upheavals of Thought. The Intelligence of Emotions. Cambridge.

Pinhard, Inga (2005): Von modernen Frauen und Früchten des Wissens. Separate Spheres und die Politik der Repräsentation in der Weltausstellung in Chicago 1893. In: Sarkowsky; Katja/Lettow, Susanne/Manz, Ulrike (Hg.): Öffentlichkeiten und Geschlechterverhältnisse. Strategien, Erfahrungen, Subjekte. Königstein (im Erscheinen).

Pauer-Studer, Herlinde (2003): Einführung in die Ethik. Wien.

Seigfried, Charlene Haddock (1993): Shared Communities of Interest: Feminism and Pragmatism. In: Hypathia. A Journal of Feminist Philosophie. Vol. 8, No.2 Spring 1993.

Schüler, Anja (2004): Frauenbewegung und soziale Reform. Jane Addams und Alice Salomon im transatlantischen Dialog; 1889-1933. Stuttgart.

Whitman, Walt (1963): Prose Works 1892. New York.

Whitman, Walt (2000): Grashalme. Stuttgart. 


\section{Rezensionen}

\section{Eva Borst: Anerkennung der Anderen und das Problem des Unterschieds. Perspektiven einer kritischen Theorie der Bildung. Hohengehren 2003}

Die Grunderfahrung der postmodernen Moderne ist nach Wolfgang Welsch die radikale Pluralität differenter Wissensformen, Sprachspiele, Lebensentwürfe und Handlungsmuster. Bestimmt aber Differenz die Breite der Lebenswirklichkeit, stellt sich in aller Schärfe die Frage, wie mit dieser Erfahrung der Verschiedenheit umzugehen ist bzw. welche Konsequenzen die Erfahrung der Andersheit für bildungstheoretische Überlegungen hat.

Vor diesem Hintergrund entwirft Eva Borst, Hochschuldozentin an der Universität Mainz, in ihrer Studie „Anerkennung der Anderen und das Problem des Unterschieds. Perspektiven einer kritischen Theorie der Bildung" ausgehend von philosophischen, psychoanalytischen und soziologischen Diskursen, Perspektiven einer kritischen Theorie der Bildung, deren zentraler Bezugspunkt die komplexe Struktur des Anerkennungsbegriffs darstellt.

Unter „Anerkennung der Anderen“ versteht Borst die „niemals abzuschließende Auseinandersetzung mit der Andersheit und Fremdheit der Anderen, die auch, aber nicht nur, im Eigenen zu suchen ist, und zwar auch jenseits der Geschlechtergrenzen“ (S. 10). Demgegenüber behandelt Borst mit dem „Problem des Unterschieds“ die Entstehungsbedingungen von Geschlechtsidentitäten innerhalb des kulturellen Systems der Zweigeschlechtlichkeit.

Reflektiert die Autorin im ersten der beiden Teile mit den Ansätzen von Hedwig Ortmann und Wiltrud Gieseke feministische Bildungstheorien und ihre Berücksichtigung der Kategorie der „Weiblichkeit“, zielt der zweite Teil in Auseinandersetzung mit Axel Honneth, Jessica Benjamin und Hans-Joachim Heydorn auf Perspektiven einer kritischen Theorie der Bildung.

Der erste Teil des Buches beginnt mit einer kurzen diskurskritischen Darstellung der Begriffsgeschichte von „Weiblichkeit“ (Kapitel 1). Die Autorin stärkt den für die Pädagogik zentralen Begriff der „geistigen Mütterlichkeit“, mit dem Helene Lang die Anerkennung der Frauen als vernunftbegabte Menschen forderte. Neuere Mütterlichkeitskonzeptionen verhindern durch die Gleichsetzung von „Mütterlichkeit“ und „Weiblichkeit“ den Blick für Geschlechterkonstruktionen jenseits der Zweigeschlechtlichkeit und fallen hinter 
das von Lang entworfene Modell zurück. Dies gilt auch für die aktuellen feministischen Ansätze von Ortmann und Gieseke, die in den Augen der Autorin zu einer Essentialisierung von Geschlecht beitragen und eine kritische Auseinandersetzung mit der symbolischen Ordnung und ihren Institutionen verhindern. Auf formaler Ebene kritisiert Borst an Ortmanns Ansatz, dass sie ihre erkenntnistheoretischen Grundlagen nicht skizziert - eine Kritik, die die Autorin auch an Gieseke übt -, auf inhaltlicher Ebene, dass die Entfaltung innerer Anlagen als zentrale Bildungsaufgabe verstanden wird (Kapitel 2). Politische und menschliche Emanzipation als Voraussetzung und Ziel aller Bildung, die notwendige Analyse gesellschaftlicher Macht- und Herrschaftsverhältnisse werden negiert. Es gehe Ortmann „,nicht um die (politische) ,Bildung von Frauen', sondern um die ,Bildung des Weiblichen', das als genuin vorausgesetzt und in spiritueller Bewusstseinserweiterung geschaut werden soll" (S. 57). Das Selbst erlebt sich demnach als anerkannt, ,wenn es im System seinen Platz ausfüllt" (S. 31). Giesekes bildungstheoretische Überlegungen intendieren in Anlehnung an das ,affidamento-Konzept“ eine Demokratisierung des Geschlechterverhältnisses (Kapitel 3). Zwei Typen von Frauenbildung sind aus Sicht Giesekes von Relevanz: die „eingreifende Bildung“ (S. 93) sowie die biographische Selbstaufklärung. Beansprucht das erste Konzept, institutionelle Bedingungen aus einer Geschlechterperspektive heraus zu verändern, so verbinden sich mit dem zweiten Konzept lebenslaufbegleitend integrierende oder spezifische Angebote, um selbstbewusstseinsstärkend, aufklärend, kritisch oder auch handlungsunterstützend zu wirken. Borst unterzieht den bildungstheoretischen Ansatz Giesekes einer systematischen Kritik. Das Versprechen Giesekes, erst die ,uneingeschränkte Akzeptanz des weiblichen Körpers" (S. 64) schenke den Frauen Freiheit und Selbstbewusstsein, verstelle den Blick für sämtliche Denaturalisierungsversuche der Geschlechterdifferenz. Das Konzept einer „eingreifenden Bildung“ (S. 93), indem Frauen ,ent-erzogen“ (S. 93) werden sollen zu einer positiven weiblichen Identifikation, lasse, so Borst, „demokratische Grundsätze von politischer Bildung hinter sich [und] missachte auch die historisch-gesellschaftliche Gewordenheit von Frauen“ (S. 94). Eine solche Bildungsvorstellung gipfelt nach Borst im Anerkennen der biologisch begründeten Differenz, und Zweigeschlechtlichkeit werde in Gesellschaft, Denken und Institutionen zementiert.

Im zweiten Teil des Buches entwickelt Borst in Auseinandersetzung mit Axel Honneth, Jessica Benjamin und Heinz-Joachim Heydorn Perspektiven einer kritischen Theorie der Bildung und reflektiert die unterschiedlichen Dimensionen der Anerkennung (Kapitel 1). Anerkennung soll nicht eindimensional im Sinne einer Anpassung an eine eindeutige Geschlechtsidentität verstanden 
werden, sondern die Analyse gesellschaftlicher Macht- und Herrschaftsverhältnisse sowie intersubjektiver Prozesse berücksichtigen.

Diese Zielsetzung begründet Borsts Interesse an Honneth, der den Anerkennungsbegriff im Rückgriff auf Hegel und Mead sehr differenziert im Verhältnis mit individuellen Identitätsbildungsprozessen und historischgesellschaftlichen Bedingungen analysiert (Kapitel 2). Im Zentrum der Überlegungen Honneths steht der moralische Konflikt, der entsteht, wenn Anerkennung gefährdet ist. Den drei Typen der Anerkennung (Liebe, Recht, Solidarität) wie der Missachtung (Vergewaltigung/Folter, Entrechtung, Beleidigung/Entwürdigung) ordnet er jeweils drei Formen der Selbstbeziehung (Selbstvertrauen, Selbstachtung, Selbstwertgefühl) und drei verschiedene Interaktionssphären zu (affektiv, kognitiv, rational gewordener Affekt), die „miteinander verschränkt sind und erst in ihrer Gesamtheit die ideale Bedingung der Möglichkeit für ein lebendiges und gleichberechtigtes Zusammenleben bilden" (S. 122).

Da allerdings geschlechtsspezifische Aspekte von Honneth nicht eigens thematisiert werden, greift Borst auf die psychoanalytische Theorie der Anerkennung von Benjamin zurück, um das Macht- und Herrschaftsverhältnis zwischen den Geschlechtern in ein komplexes Strukturmodell der Anerkennung zu integrieren (Kapitel 3). Zentrales Moment der Theorie Benjamins ist die wechselseitige Anerkennung zweier Subjekte, deren Subjektsein durch den Prozess der Anerkennung gesichert ist. In Anlehnung an Hegels Dialektik versteht Benjamin die Konflikte zwischen der Behauptung des Selbst und dem Bedürfnis nach dem Anderen als ein „Paradoxon der Anerkennung“, in dessen Spannungsgefüge sich jedes Individuum jenseits der Geschlechtergrenzen zu halten habe. Um selbst anerkannt zu sein, muss ich auch den Anderen als ursprüngliches Selbst - und nicht nur für mich seiend - anerkennen. Benjamins Aufmerksamkeit gilt der Struktur von Herrschaft und Unterwerfung zwischen den Geschlechtern. In ihrer Suche nach Antworten auf die Frage, warum Frauen sich freiwillig unterwerfen bzw. ihnen (weibliche) Subjektivität verwehrt wird, greift sie auf die Objektbeziehungstheorie Nancy Chodorows und die Entwicklungstheorie Donald W. Winnicotts zurück.

Benjamins Analyse intrapsychischer Prozesse und intersubjektiver Anerkennung werden von Borst im letzten Kapitel mit Heydorns kritischer Bildungstheorie weitergedacht (Kapitel 4). Seine Überlegungen zur Konstitution des Subjekts und zu den immanenten Widersprüchen von Macht- und Herrschaftsverhältnissen in Erziehungs- und Bildungsprozessen ergänzen die Benjaminsche Subjektkonzeption um den Aspekt der subjektiven Zerrissenheit. Anerkennung vollzieht sich nicht nur intersubjektiv, sondern muss ,auch gattungsgeschichtlich Gestalt im Bildungsprozess gewinnen, um sich auf gesell- 
schaftlicher Ebene institutionalisieren zu können“" (S. 181). Bildung ist demnach ein Prozess, in dem der Mensch sich als Subjekt begreift, das kritisch und handelnd in die Geschichte eingreifen kann.

Aus ihrer Relecture Heydorns mit Adorno, Foucault, Butler und Žižek ergibt sich für Borst zum einen, dass eine aus dem Prozess hervorgehende „Kultur der Anerkennung“ (S. 225) der Offenheit gegenüber Unbekanntem und Nicht-Identischem, des Aushaltens von Entfremdung sowie des Respekts vor der Andersheit im Anderen bedarf. Zum anderen vermitteln Anerkennungsprozesse die Sensibilität für Ein- und Ausschlussmechanismen und schärfen den Blick für das in hegemonialen Strukturen und Diskursen Unsichtbare oder Unausgesprochene. Die Reflexion von Anerkennungsprozessen könnte ein (politisches) Handeln ermöglichen, das hegemoniale Strukturen subvertiert und Handlungsalternativen eröffnet.

Hervorzuheben ist das Anliegen des Buches, Anerkennung als zentrale Kategorie erziehungs- und bildungstheoretischer Überlegungen zu stärken. „[I]n glücklichen Momenten“, so Borst, „fallen Bildung und Anerkennung in eins, mehr noch können durch die Erfahrung von Nicht-Identiät prinzipiell Veränderungsprozesse in Gang gesetzt werden, die in intersubjektiver Anerkennung münden und (...) zumindest ein gesellschaftliches sowie politisches Bewusstsein hervorzurufen (...) vermögen" (S. 210). Auch das mögliche Scheitern von Bildung- und Anerkennungsprozessen behält die Autorin im Auge, denn der „Ausgang von Bildung [ist] ebenso ungewiss wie uneindeutig“ (S. 210). Kritisch anzufragen bleibt allerdings, in welchem genauen Verhältnis Bildung und Anerkennung stehen. Heißt es einerseits, dass Bildung und Anerkennung in eins fallen können, sieht Borst andererseits Bildung als Voraussetzung für Anerkennung an (S. 179, S. 217). Die differenzierte Aufarbeitung der erkenntnistheoretischen Theorieansätze der einzelnen Ansätze verdienen Anerkennung, die eigene kritische Reflexion der Autorin, wie die von ihr intendierte „Kultur der Anerkennung“ inhaltlich und methodisch etabliert werden kann, bleiben im Hintergrund. Ob die Kritik an kanonisierten Bildungsinhalten und das Plädoyer für die Methode des Sokratischen Gesprächs für die Beantwortung der Frage, wie das einzelne Subjekt in lebensgeschichtlichen Lernprozessen zur Anerkennung befähigt werden kann, ausreichen, darf bezweifelt werden. Kann eine kritische Bildungstheorie nur noch ihre Funktion bestimmen, ohne konkrete Bildungsinhalte benennen zu können?

Bemerkenswert ist das Anliegen der Autorin, die verschiedenen Theorieansätze stets mit der Geschlechterfrage zu konfrontieren, um die Frage, wie geschlechterdichotome Zuschreibungen destabilisiert werden und die Vielfalt von Lebensformen Anerkennung finden können, zu beantworten. Gerade im 
Hinblick auf bildungstheoretische Überlegungen müsste Differenz jedoch nicht allein auf das Geschlechterverhältnis begrenzt sein, sondern die Berücksichtigung von Rasse, Ethnizität, Nationalität, Sexualität und sexuelle Orientierung einschließen, um eine Perspektivenverengung zu verhindern.

Christiane Micus-Loos

\section{Corinna Seith: Öffentliche Interventionen gegen häusliche Gewalt. Zur Rolle von Polizei, Sozialdienst und Frauenhäusern. Campus 2003.}

Bei der vorliegenden Arbeit handelt es sich um eine Dissertation, die im Rahmen des Schweizerischen Nationalen Forschungsprojektes 40 „Gewalt im Alltag und organisierte Kriminalität“" (April 1997 bis Januar 2001) entstanden ist. Die öffentliche Interventionspraxis gegen häusliche Gewalt wird mit Blick auf die Rolle von Polizei, Sozialdienst und Frauenhäusern im Kanton Freiburg untersucht. Zurückgeführt wird dieses Forschungsinteresse zum einen auf eine seit den 1970er Jahren angeführte feministische Gewaltdiskussion, die den Zusammenhang zwischen Frauenmisshandlung, strukturellen und kulturellen Faktoren untersucht und das Argument des Schutzes der Privatsphäre kritisiert. Vor diesem Hintergrund ist zu fragen, ,inwieweit die feministische Definition, die Gewalt in Ehe und Partnerschaft als asymmetrische Machtausübung im Geschlechterverhältnis konzeptionalisiert, institutionelles Handeln prägt." (S. 19) Zum anderen konstatiert die Autorin ein erhebliches Forschungsdefizit im Hinblick auf gesicherte statistische Grundlagen, die u.a. über ,die Struktur der Klientel, das Verhältnis von Opfer und Täter, die Phänomenologie der Gewalt“" (S. 19) Auskunft geben würden. Ziel der Untersuchung ist, für die Praxis eine Wissensbasis im Umgang mit häuslicher Gewalt $\mathrm{zu}$ schaffen und gleichzeitig Grundlagenforschung auf diesem Gebiet zu betreiben.

Nach einem knapp 30-seitigen Überblick zum aktuellen Forschungsstand, aus dem das zuvor konstatierte Forschungsdefizit auf unterschiedlichen Ebenen deutlich hervorgeht und zum Ausdruck kommt, dass sich von häuslicher Gewalt betroffene Frauen durch die öffentlichen Institutionen wenig unterstützt fühlen, werden Theorie- und Methodenwahl begründet. Um die Handlungsebene professioneller AkteurInnen, Strukturbedingungen verschiedener Organisationen und die Interaktionsprozesse zwischen den Organisationen in den Blick zu nehmen, greift die Autorin auf die in der Theorietradition des 
Symbolischen Interaktionismus stehende Social Worlds/Arena Theory zurück. In diesem Zusammenhang werden Arenen als soziale Orte verstanden, ,an denen RepräsentantInnen verschiedener sozialer Welten/Felder in Aushandlungsprozesse zum Beispiel rund um das Thema Gewalt in Ehe und Partnerschaft verwickelt werden." (S. 50f.) Zur Herausarbeitung charakteristischer Merkmale des jeweiligen Feldes im Umgang mit häuslicher Gewalt werden bei der Polizei strukturelle, rechtliche und kulturelle Faktoren untersucht, beim Sozialdienst gesellschaftliche, rechtliche, institutionelle sowie regionale Bedingungen und beim Frauenhaus bilden die Leitideen der Frauenhausbewegung (Schutz und Sicherheit/Hilfe zur Selbsthilfe/Prävention und Gesellschaftsveränderung) den analytischen Rahmen. Zusätzlich wird Giddens' Theory of Structuration in die Überlegungen mit einbezogen, vor dessen Hintergrund u.a. die zentrale Frage nach den unintendierten Folgen professionellen Handelns aufgeworfen wird, so z.B. im Hinblick auf eine Reprivatisierung von Gewalt oder aufgrund institutionell unterschiedlich verteilter Definitionsmacht in der Institutionen-Arena. Bei den relativ knapp gehaltenen Ausführungen wird insgesamt zu wenig deutlich, wie sich die gewählten Theorien aufeinander beziehen und welche Ebenen sich mittels welcher Methoden untersuchen lassen. Gerade die Auseinandersetzung mit Giddens würde dies jedoch nahelegen, da er selber ausführliche Überlegungen dazu anstellt, wie die empirische Umsetzung seiner Strukturierungstheorie aussehen könnte.

Um sowohl die Handlungspraxis zu untersuchen als auch das Ausmaß, in welchem die verschiedenen Institutionen mit häuslicher Gewalt konfrontiert sind, wird ein multimethodologisches Untersuchungsdesign gewählt. Unterschiedliche Materialien (300 Akten, 24 qualitative Interviews mit Professionellen, zahlreiche ExpertInneninterviews und vier Interviews mit von Gewalt betroffenen Frauen) werden mittels verschiedener Methoden analysiert. Die Akten werden sowohl einer quantitativen Inhaltsanalyse unterzogen, um Struktur und Häufigkeit der Gewaltformen sichtbar zu machen, als auch nach grounded theory und Objektiver Hermeneutik ausgewertet. Dieselben qualitativen Methoden finden auch bei der Analyse der Interviews ihre Anwendung und sollen die Rekonstruktion institutionalisierter Handlungslogiken ermöglichen. Die vier Fallrekonstruktionen mit von Gewalt betroffenen Frauen sind im Anhang abgedruckt und bilden die Komplexität der Fälle ab. Die Datenerhebung und Stichprobenbildung für die Aktenanalyse wird sehr ausführlich dargestellt. Bei einem so vielschichtigen Untersuchungsdesign wäre es allerdings hilfreich gewesen, eine ähnlich vertiefte methodische und methodologische Diskussion in Bezug auf die gewählten Verfahren zu führen. So bleibt insbesondere die Verbindung zwischen grounded theory und Objektiver Hermeneutik nicht eindeutig nachvollziehbar. 
In den drei folgenden Kapiteln werden die umfangreichen und detaillierten Ergebnisse zunächst getrennt nach den Institutionen Polizei, Sozialdienst und Frauenhaus präsentiert: Der institutionelle Rahmen für die polizeiliche Interventionspraxis stütze sich im Wesentlichen auf das Strafgesetz und die Strafprozessordnung. In Abhängigkeit der Schwere häuslicher Gewalt werde ein Vergehen entweder als strafrechtlich relevantes Delikt oder als soziales Problem eingestuft. Die gesetzlichen Rahmenbedingungen lassen bei Körperverletzung, Waffenbesitz des Täters und bei Freiheitsberaubung eine Kriminalisierung von Gewalt gegen Frauen zu. Werden vor diesem Hintergrund die Ergebnisse der quantitativen Inhaltsanalysen verglichen, so zeige sich eine große Diskrepanz hinsichtlich der Interpretation der dokumentierten Realitäten. Gewalt in Ehe und Partnerschaft sei vielgestaltig und trete oft in Kombination mit physischer/sexueller und psychischer/ökonomischer Gewalt auf. Aufgrund der institutionenspezifisch geprägten Wahrnehmung werde diese Heterogenität an Gewaltformen jedoch nicht sichtbar. Dies habe Auswirkungen auf die Interventionspraxis. Die Schutzfrage der Frau bleibe in der Regel ungeklärt, polizeiliche Intervention ziele auf Schlichtung und Vermittlung bei sogenannten „Familienstreitigkeiten“ und im Zuge dessen werde Gewalt in Ehe und Partnerschaft nicht als Rechtsverletzung sondern als Privatsache behandelt.

Ähnlich wie bei der Polizei fehle auch beim Sozialdienst eine Statistik, die über Ausmaß, Formen und Kontext häuslicher Gewalt Auskunft gibt und so zu einer differenzierten Wahrnehmung unterschiedlicher Gewaltformen beitragen würde. Besonders sichtbar würden durch die Analyse der Sozialdienstakten diejenigen Gewaltformen, die in Zusammenhang mit Scheidung/ Trennung und ökonomischen Autonomiebestrebungen der Frauen stehen. Von Seiten des Sozialdienstes werde Gewalt in der Regel als Beziehungsproblem interpretiert und geschlechtsneutral formuliert („Streit zwischen Eheleuten"), was einer Umdeutung des asymmetrischen Macht- bzw. Geschlechterverhältnisses gleichkomme. Interessanterweise stehe dies im Gegensatz zu dem hohen Problembewusstsein der SozialarbeiterInnen hinsichtlich der ökonomischen Ausbeutung von Frauen. Dieses Wahrnehmungsmuster führe dazu, dass materielle Hilfestellungen im Vordergrund stehen, eine Form von Hilfe, die eng mit den strukturellen Aufgaben des Sozialdienstes in Verbindung stehe und infolge dessen die dahinter liegende Gewaltproblematik selten aufgedeckt würde.

Der institutionelle Kontext beim Frauenhaus orientiere sich am Gleichheitsgrundsatz der Frauenhausbewegung. Die Arbeit des Frauenhauses basiere auf dem „Komm-Prinzip“, gewähre den Frauen größtmögliche Selbstbestimmung und biete einen sicheren Zufluchtsort. Das Frauenhaus sei, wie die 
anderen Institutionen auch, mit verschiedenen Formen von Gewalt konfrontiert. Ein auffallendes Ergebnis sei, dass sich v.a. verheiratete Frauen an das Frauenhaus wenden. Getrennte bzw. geschiedene Frauen wenden sich eher an die Polizei oder den Sozialdienst. Gewalt werde im Frauenhaus zu einem Großteil als Rechtsverletzung konzipiert. Die Frauen erhalten Informationen über das Opferhilfegesetz, über zivil- und strafrechtliche Möglichkeiten, es werde auf AnwältInnen, juristische Instanzen und die Polizei verwiesen. Durch offene Beratungstätigkeit werde für die von Gewalt betroffenen Frauen eine möglichst realistische Grundlage zur Entscheidungsfindung für weitere Schritte geschaffen.

Im nächsten Kapitel stehen nicht mehr die einzelnen Institutionen im Zentrum, sondern „Gewalt in Ehe und Partnerschaft in der Institutionen-Arena“. Um interinstitutionelle Prozesse zu analysieren, greift die Autorin auf Annahmen der Interagency-Literatur und Aspekte aus organisationssoziologischer Perspektive zurück. Anhand zahlreicher Interviewausschnitte wird die Zusammenarbeit zwischen den verschiedenen Institutionen illustriert, so zwischen Polizei und Frauenhaus, Sozialdienst und Frauenhaus, Frauenhaus und Justiz. Es wird aufgezeigt, dass sich interinstitutionelle Kontakte im konkreten Fall konflikthaft oder ambivalent gestalten können, da unterschiedliche Logiken aufeinander prallen.

Im Schlusskapitel werden die wichtigsten strukturellen Probleme zusammengefasst und dabei wird nochmals die Frage aufgeworfen, „ob sich in der konkreten Praxis doch das alte Deutungsmuster durchsetzt, dass der Staat sich nicht in interne Angelegenheiten einzumischen hat" (S. 237). Da bereits zu Beginn der Arbeit als unbeabsichtigte Handlungsfolge die Reprivatisierung von Gewalt angesprochen worden ist, erstaunt es, dass darauf keine Antwort formuliert wird. Ein Grund mag darin liegen, dass der Analyse professionellen Handelns kein theoretisches Konzept zugrunde liegt, um den im Kern „feministischen" Blick auf dieses Thema systematisch anzugehen. Somit bleiben die Auswirkungen der am Anfang der Arbeit angesprochenen „strukturellen und kulturellen Faktoren von Frauenmisshandlungen “ auf institutioneller Ebene bis zum Schluss unscharf. Schließlich werden zur Überwindung institutioneller Hürden folgende Maßnahmen genannt: Sammeln von Informationen zur Sichtbarmachung von Gewalt in Ehe und Partnerschaft; Sanktionierung der Täter durch konsequentere Strafverfolgung; Trainingsprogramme für gewalttätige Partner; frühzeitige Intervention und pro-aktive Unterstützung der Frauen. Ob mittels der Umsetzung der hier genannten Maßnahmen wirklich asymmetrischen Macht- und Geschlechterverhältnissen entgegengewirkt werden kann, bleibt allerdings fraglich. 
Trotz einzelner theoretischer und methodischer Unschärfen gelingt es der Autorin, vor dem Hintergrund einer empirisch breit angelegten Untersuchung die Komplexität im Umgang mit häuslicher Gewalt sichtbar sowie interinstitutionelle Prozesse der Analyse zugänglich zu machen. Das ambitionierte Unterfangen setzt somit einen Meilenstein zur Erforschung eines gesellschaftlich hoch relevanten Themas, das sich bis anhin dieser Problematik nicht in diesem Umfang gestellt hat.

Barbara Fontanellar.

\section{Heide von Felden: Bildung und Geschlecht zwischen Moderne und Postmoderne. Zur Verknüpfung von Bildungs-, Biographie- und Genderforschung. Opladen: Leske + Budrich 2003 (Band 21 der Reihe: Studien zu Erziehungswissenschaft und Bildungsforschung)}

Heide von Felden greift ein altes Thema der pädagogischen Frauenforschung auf, die Frage des androzentrischen Charakters von Bildung und seiner Veränderung. Anders als Ortmann oder Schaeffer-Hegel in den 1980er Jahren geht es aber nicht um das Entwickeln einer sog. „Weiblichen Bildungstheorie", sondern um eine weitgehend deskriptiv angelegte Integration der Konzepte ,Geschlecht" und ,Bildung' in einer primär empirischen Perspektive. Felden hinterfragt zwar auch „die behauptete Universalität von Bildung“ (S. 9), dies aber nicht durch die Kritik des Bildungsdiskurses, von Bildungsprozessen oder durch das Entwerfen eines normativen Gegenkonzepts, sondern durch die kritische Fortschreibung eines aktuellen methodischen Zugangs zur Bildungsforschung, nämlich der qualitativen Biographieforschung.

Die Habilitationsschrift geht in einem Dreischritt vor. Zuerst wird der Stand der Forschung diskutiert und ein Vorverständnis entwickelt (Kap. 2). Mit diesem wird empirisches Material (Kap. 3 und 4) exemplarisch ausgewertet und schließlich werden als Ergebnisse „Bausteine einer Bildungstheorie, die Geschlecht und Biographie integriert“ (Kap. 5), vorgestellt.

Die interessante Diskussion des Stands der Bildungstheorie und der Geschlechterforschung führt Felden zu einer geschlechtersensiblen Bildungstheorie, die sich auf die interpretative Soziologie stützt. Wichtige Stationen ihres Weges dorthin sind, neben historischen Ausflügen, bildungstheoretische Schriften von Scherr, Marotzki, Koller und Alheit sowie die für die Frauenforschung zentralen Problematiken der Überwindung der Defizitperspektive 
auf Weiblichkeit, die Gleichheit/Differenz-Debatte sowie neue Veröffentlichungen zur De-/Konstruktion von Geschlecht. Als sog. moderne Positionen werden Adorno für die Bildungstheorie und Benhabib für die Geschlechtertheorie andiskutiert; als postmoderne Positionen Lyotard für die Bildungstheorie und Butler für die Geschlechtertheorie. Feldens Resümee, Geschlecht wie Bildung seien „kulturelle Konstrukte“ (vgl. S. 120) mit verschiedenen Dimensionen (,Denkform“, „Vergesellschaftung“, „Kulturaneignung“, „Norm“, „Kritik“), bleibt gegenüber den diskutierten Schriften dann allerdings begrifflich unklar und es wird auf die „Wirklichkeitskonstruktionen der Individuen“ (S. 124) verwiesen, die zu untersuchen seien.

Schwerpunkt der Schrift ist letztlich die Begründung und Darstellung einer qualitativ orientierten Biografieforschung, die geschlechtssensibel Bildungsprozesse erfassen will. Dieser Ansatz geht methodisch von der Analyse der subjektiven Welt- und Selbstdeutungen aus, in bzw. aus denen heraus gesellschaftliche Strukturen erschlossen werden. Die Subjekte erscheinen in dieser Perspektive als einerseits an die gesellschaftlichen Wissensbestände gebunden, andererseits als frei - in ihrem „Doing Biography“ bzw. „Doing Gender".

Felden hat 20 narrative Interviews mit Studentinnen des 3./4. Semesters eines weiterbildenden Studiengangs Frauenstudien durchgeführt. Leider werden nur drei Fälle dargestellt, diese allerdings ausführlich und anschaulich. Ausgewertet wurden die Interviews einerseits i.S. der Hinweise von Fritz Schütze, andererseits hinsichtlich der im Vorverständnis gewonnenen Konzepte von ,Bildung' und ,Geschlecht' als normativ-kulturelle Konstruktionen. Bezüglich der Verbindung von Theorie und Empirie stützt sich Felden vor allem auf Marotzki und Dausien.

Am ersten Interview zeigt Felden, wie der Studiengang eine biografisch angelegte Resistenz gegenüber Bildungsprozessen, die mit einer polarisierenden Sicht auf Männer und Frauen einhergeht, nicht auflösen kann und ein instrumentelles Lernen vorherrscht. Neben der Beschreibung des Falls bewertet Felden meines Erachtens nun aber auch, wenn sie z.B. schreibt: „Die Überwindung dieser gesellschaftlich vorherrschenden und die hierarchische Trennung der Geschlechter bestätigenden Denkweise würde vermutlich einen Bildungsprozess darstellen, der Christiane Reimann (die Interviewte, F.B.) zu sich selbst führen und die Integration ihrer verschiedenen Lebensphasen ermöglichen könnte" (S. 182f.). Die Auswertung des zweiten Interviews schildert dann den Beginn eines Bildungsprozesses im Sinne Feldens. Die Interpretation des Interviews skizziert das Ringen einer ,innerlich zerrissenen“ Frau um private und berufliche Handlungsmöglichkeiten, das sich auch in deren Selbstbildern spiegelt. Im dritten Interview schließlich wird ein Wand- 
lungs- bzw. „Bildungsprozess“ beschrieben, wo meines Erachtens wiederum die Integration, in diesem Falle von Lebensweise und Berufswunsch, als Norm erscheint - die aber von Felden nicht expliziert diskutiert wird. Hierin läge aber m.E. das erste zu diskutierende Problem einer Verbindung von Bildungstheorie zu einer sich vornehmlich deskriptiv verstehenden Soziologie.

Das zweite Problem sehe ich darin, dass die anschaulichen Schilderungen der Fälle theoretisch nur wenig fruchtbar zu machen sind. In Feldens Abschlusskapitel (vgl. S. 239-254), das die Ergebnisse der Fälle für die Bildungstheorie darstellt, werden eher Allgemeinplätze angeboten: Bildungsprozesse spielen für die Transformation von Lebensverläufen und ihrer subjektiven Deutung ein große Rolle. Geschlechtstypische Zuschreibungen engen die Handlungspotentiale ein, so die gesellschaftliche Anerkennung. Oder etwas länger: „Die empirische Untersuchung hat gezeigt, dass die Konstruktion von Lern- und Bildungsprozessen über die Konstruktion der Erfahrungsaufschichtung und das Selbst- und Weltbild von der Auseinandersetzung mit gesellschaftlichen, geschlechtstypischen und geschlechtsbedingten Zuschreibungen abhängt, so dass behauptet werden kann, dass geschlechtliche Zuschreibungen Lernen und Bildung in bestimmter Weise beeinflussen." (S. 249) In solchen Formulierungen rächt sich dann m.E. das Trennen der Warum- von WieFragen, aber auch das begriffliche Ausklammern von makrotheoretischen Fragen in der Methodologie, aber auch beim Geschlechtsbegriff. Geschlecht als „Zuschreibungen an Menschen“ (S. 121, S. 140 oder S. 249) anzusehen, übergeht, dass Geschlechtlichkeiten in Institutionen, gesellschaftlichen Sphären, Diskursformationen, sog. Wohlfahrtsstaatsregimes etc. eingelassen sein können - und dies weitgehend unabhängig von Geschlechtszuschreibungen eines/r AkteurIn. Bildungstheoretisch halte ich diese Annahme für grundlegend, sollen auch geschlechtsneutrale Formulierungen, z.B. in Lehrplänen oder auch in der Bildungsphilosophie gegendert werden.

Nach den interessanten theoretischen Diskussionen zu Beginn und dem anspruchsvollem Titel enttäuscht mich so das Buch am Ende. Methodische Grenzen sehe ich darin, dass aus den drei dargestellten Interviews keine „Arten (Typen?, F.B.) von biographischen Lern- und Bildungsprozessen ermittelt werden“ (S. 13) können - zumal die Lesenden über die anderen geführten Interviews und damit über die Auswahl wenig erfahren (vgl. S. 145 und S. 153). Zudem ist zu fragen, warum nur Frauen befragt wurden, wenn doch Geschlecht bzw. Gender theoretisiert werden soll. 


\section{Ankündigung \\ Jahrbuch Band 2: \\ Geschlechtertypisierungen im Kontext von Familie und Schule}

Der zweite Band der Jahrbuchreihe zur Frauen- und Geschlechterforschung in der Erziehungswissenschaft beschäftigt sich mit Geschlechterbildern und geschlechtstypischen Erwartungen, mit denen Kinder und Jugendliche sich im Prozess des Aufwachsens auseinandersetzen müssen.

Die Herausgeberinnen der Jahrbuchreihe verfolgen dabei zwei zusammenhängende Anliegen: Erstens geht es um das Ein- und Zusammenwirken von Schule und Familie auf Kinder und Jugendliche in Bezug auf Geschlechtstypisierungsprozesse. Dabei sollen die Reflexion von Zusammenhängen und die Frage nach dem Zusammenwirken der einzelnen Einflussfaktoren im Vordergrund stehen.

Zweitens möchte der Band (wie die gesamte Reihe) zur Repolitisierung der erziehungswissenschaftlichen Diskurse über Bildung, Sozialisation und Wissenschaft und zur Präzisierung des Verhältnisses von pädagogischen und gesellschaftlichen Denkfiguren beitragen.

Wir bitten um die Ankündigung von Beiträgen oder Forschungsberichten (mit kurzem Exposé) bis Ende April 2005 und um die Abgabe der Artikel bis Ende Juli 2005, damit genügend Zeit für das Reviewverfahren bleibt.

Als Herausgeberinnen von Band 2 sind Sabine Andresen und Barbara Rendtorff verantwortlich. Abstracts und Texte per Email bitte an:

sabine.andresen@uni-bielefeld.de und BarbaraRendtorff@compuserve.de. 


\section{Ankündigung Jahrbuch Band 3: „Bildung - Macht - und Öffentlichkeit“}

„Bildung - Macht - Öffentlichkeit“ umreißt ein Themenfeld, das angesichts des zu beobachtenden Systemwechsels in Politik, Wissenschaft, Kultur und Sozialem unter geschlechtertheoretischer Perspektive neu diskutiert werden sollte. Da pädagogische und erziehungswissenschaftliche Fragestellungen von diesen Umstrukturierungen nicht unberührt bleiben, sind Fragen wie die folgenden von Interesse: Was bedeutet eine kritische Frauen- und Geschlechterforschung im System der Hochschulen? Was bedeuten die Begriffe Macht und Kritik im Hinblick auf Geschlecht, und welche Konsequenzen zeitigen sie in den Bildungsinstitutionen? Welche Auswirkungen hat die Ökonomisierung der Bildung auf die Geschlechterfrage? In welchem Verhältnis stehen Bildung, Geschlecht und Macht? Wie verändern sich die Bezugsgrößen Öffentlichkeit und Privatheit angesichts neoliberaler (Wirtschafts-)Politik?

Als Herausgeberinnen von Band 3 sind Rita Casale und Eva Borst verantwortlich. Abstracts und Texte per Email bitte an:

rcasale@paed.unizh.ch und borst@mail.uni-mainz.de 


\section{Über die AutorInnen}

Andresen Sabine, Dr. Professorin für Allgemeine Erziehungswissenschaft an der Universität Bielefeld. Forschungsgebiete: Erziehungswissenschaftliche Kindheits- und Jugendforschung, Historische Geschlechterforschung, Historische Bildung- und Sozialforschung, Geschichte und Kritik der Reformpädagogik (Ellen Key), Sozial- und kulturtheoretische Perspektiven der Erziehungswissenschaft, Bildungstheorien, Wissenstransfer im erziehungswissenschaftlichen Kontext

Bönold, Fritjof, Dr., Nürnberg; Forschungsgebiete: Allgemeine Geschlechterforschung, Gender Mainstreaming, Organisationspädagogik, Montessoripädagogik.

Breitenbach, Eva, PD, z.Zt. Vertretungsprofessorin an der PH Freiburg. Forschungsgebiete: konstruktivistische Geschlechterforschung, Jugendforschung, rekonstruktive Methoden.

Casale, Rita, Dr., Oberassistentin am Pädagogischen Institut der Universität Zürich, Forschungsgebiete: Vergleichende Bildungsgeschichte, Erziehungsphilosophie und Geschlechterforschung.

Fontanellaz, Barbara, lic. phil.; Pädagogisches Institut der Universität Zürich. Forschungsgebiete: Jugend und Politik; Jugendprotest und politischer Extremismus; Jugendkriminalität.

Forster, Edgar, Dr. A. Univ.-Prof., lehrt Erziehungswissenschaft an der Universität Salzburg. Forschungsgebiete: Allgemeine Pädagogik, Gender Studies.

Kampshoff, Marita, Dr.; Forschungsgebiete: Geschlechterforschung in der Schulpädagogik, Schulleistungsforschung und Jugendforschung. Lebt und forscht zur Zeit in Österreich.

Larcher, Sabina, Dr. phil.; wissenschaftliche Mitarbeiterin am Pädagogischen Institut der Universität Zürich und der Pädagogischen Hochschule Aargau. Forschungsgebiete: Bildung, Profession und Geschlecht, Geschlechter- 
forschung im Kontext des Lehrberufs, Lehrer/innenbildungsforschung in synchroner wie diachroner Perspektive, Zusammenhang von Bildungsforschung und Bildungspolitik.

Macha, Hildegard, Dr. Professorin für Pädagogik an der Universität Augsburg, Forschungsgebiete: Außerschulische Jugendbildung und Erwachsenenbildung. Vorsitzende der Sektion Frauen- und Geschlechterforschung in der DGfE.

Maurer, Susanne, Prof. für Erziehungswissenschaft mit Schwerpunkt Sozialpädagogik an der Universität Marburg. Forschungsgebiete: Frauen- und Geschlechtergeschichte der Sozialen Arbeit, Feministische Wissenschafts- und Erkenntniskritik, Soziale Bewegungen, Soziale Arbeit und gesellschaftliches Gedächtnis.

Micus-Loos, Christiane, Dr.; Institut für Allgemeine Pädagogik der Humboldt-Universität zu Berlin. Forschungsgebiete: Geschlechterforschung, Biographie- und Generationenforschung, Psychoanalytische Pädagogik, Qualitative Forschungsmethoden.

Moser, Vera, Dr. Professorin für Allgemeine Heil- und Sonderpädagogik an der Justus-Liebig-Universität Giessen, Forschungsgebiete: sonderpädagogische Theoriebildung, Historiographie der Sonderpädagogik, Professionsforschung, Geschlechterforschung.

Pinhard, Inga, MA, Wissenschaftliche Mitarbeiterin am Institut für Allgemeine Erziehungswissenschaft der J.W. Goethe Universität Frankfurt am Main. Forschungsgebiete: pädagogische Geschlechterforschung, Theorien ästhetischer Bildung, Pädagogik und Ethik, Ritualforschung.

Prengel, Annedore, Dr. Professorin für Erziehungswissenschaft an der Universität Potsdam, Forschungsgebiete: Heterogenität in der Bildung, Grundschulpädagogik, Anfangsunterricht, soziales Lernen und Integration Behinderter, Menschenrechtsbildung, Pädagogische Diagnostik.

Rendtorff, Barbara, PD, z.Zt. Vertretungsprofessorin an der Universität zu Köln. Forschungsschwerpunkte: Theorie der Geschlechterverhältnisse, Tradierung von Geschlechterbildern im Prozess des Aufwachsens; Geschlechterdimensionen in der Schule und in pädagogischen Kontexten. 


\section{Bezugsmöglichkeiten}

\section{Jahrbuch Frauen- und Geschlechterforschung in der Erziehungswissenschaft}

Das „Jahrbuch Frauen- und Geschlechterforschung in der Erziehungswissenschaft" basiert auf einer wichtigen wissenschaftlichen und politischen Entwicklung. Die Frauen- und Geschlechterforschung in der Erziehungswissenschaft hat wesentlich dazu beigetragen, dass der Einfluss von Geschlecht und Geschlechterdifferenz nicht mehr ignoriert werden kann. Das Jahrbuch ist vor diesem Hintergrund konzipiert und stellt sich zwei zentralen Aufgaben: Es will erstens die Geschlechterforschung in der Erziehungswissenschaft kontinuierlich dokumentieren und zweitens ein innovatives Forum für wissenschaftliche Diskurse schaffen. Alle Beiträge werden nach dem internationalen Standard für Review-Zeitschriften begutachtet.

Das Jahrbuch erscheint jährlich im Mai mit einem Umfang von rund 180 Druckseiten. Der Ladenpreis beträgt in der Regel 19,90 Euro.

Der Subskriptionspreis liegt bei 17,90 Euro je Jahrbuch, bzw. 2 Euro unterhalb des Ladenpreises. Die Subskription erstreckt sich automatisch immer über mindestens zwei aufeinander folgende Jahrbücher und verlängert sich jeweils um ein weiteres Jahr, wenn nicht auf Jahresende schriftlich an den Verlag gekündigt wird.

Bei Direktbestellungen an den Verlag Barbara Budrich verstehen sich die Preise zuzüglich Porto.

Bestellungen an Ihre Buchhandlung oder direkt an

\section{Verlag}

Barbara Budrich

Publishers

Stauffenbergstr. 7. D-51379 Leverkusen Opladen ph +49.2171.344.594 • Fax+49.2171.344.693• info@budrich-verlag.de

2963 London Wall. Bloomfield Hills. MI 48304. USA

ph+1.248.322.4726• info@barbara-budrich.net

www.budrich-verlag.de 


\section{The end of the male bread-winner model?}

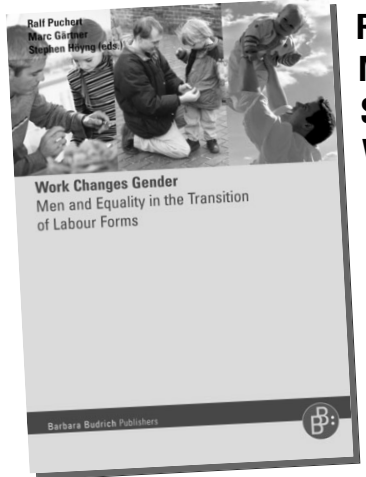

Ralf Puchert

Marc Gärtner

Stephan Höyng (eds.)

Work Changes Gender

Men and Equality in the Transition of Labour Forms

Preface by Michael Kimmel

2005. 202 pp. Pb $19.90 €$

ISBN 3-938094-13-3

Hardcover $39.90 €$

ISBN 3-938094-14-1

Changes in the work sphere and the "vanishing" of standard work may lead to the end

of the old bread-winner type of masculinity. But where "new men" try to balance work

and life or show caring activities, they face obstacles-i.e., in organisations or on play-

grounds. The book explains the ongoing changes in detail, shows ways how men deal

with them and gives recommendations how to achieve gender equality by including a

perspective on men.

At your local bookshop or contact:

\section{Verlag Barbara Budrich}

Barbara Budrich Publishers

Stauffenbergstr. 7.

D-51379 Leverkusen Opladen

Tel. +49.(0)2171.344.594.

$\mathrm{Fax}+49(0) 2171.344 .693$.

info@budrich-verlag.de
U.S.-office:

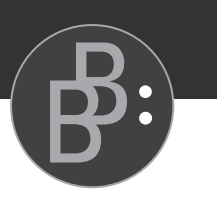

Renate Schnelle-Gotschke. 2963 London Wall. Bloomfield Hills.

MI 48304, U.S.A. ph +1 (0)248.322.4726. info@barbara-budrich.net. 


\section{Gender Mainstreaming in Europe}

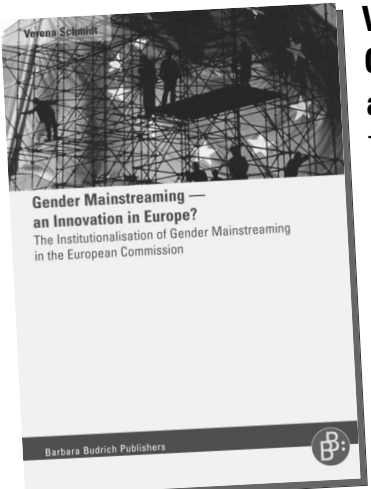

\section{Verena Schmidt \\ Gender Mainstreaming - an Innovation in Europe?}

The Institutionalisation of Gender Mainstreaming

in the European Commission

Preface by llse Lenz

2005. 272 pp. Pb 36. $-€$

ISBN 3-938094-28-1

Hardcover 48. $-€$

ISBN 3-938094-29-X

The concept of gender mainstreaming has experienced an unexpected boom in the European Union and beyond since the United Nations World Conference of Women in Beijing in 1995. Starting from the evolution of gender mainstreaming, this book examines the extent to which gender mainstreaming can be regarded as an innovation and as an institution in a complex organisation like the European Commission. By ensuring that the effects on both genders of all policies and organisational processes are taken into account, gender mainstreaming seeks to bring what are often marginalised as "women's concerns" into the mainstream of the analysis. Gender mainstreaming is often regarded as a paradigm shift compared to previous concepts of equal treatment and positive action programmes.

At your local bookshop or contact:

\section{Verlag Barbara Budrich}

\section{Barbara Budrich Publishers}

Stauffenbergstr. 7.

D-51379 Leverkusen Opladen

Tel. +49.(0)2171.344.594.

$\mathrm{Fax}+49(0) 2171.344 .693$.

info@budrich-verlag.de
U.S.-office:

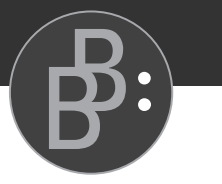

Renate Schnelle-Gotschke. 2963 London Wall. Bloomfield Hills. MI 48304, U.S.A. ph +1 (0)248.322.4726. info@barbara-budrich.net. 